\title{
STRONG ILL-POSEDNESS OF THE INCOMPRESSIBLE EULER EQUATION IN BORDERLINE SOBOLEV SPACES
}

\author{
JEAN BOURGAIN AND DONG LI
}

\begin{abstract}
For the $d$-dimensional incompressible Euler equation, the standard energy method gives local wellposedness for initial velocity in Sobolev space $H^{s}\left(\mathbb{R}^{d}\right), s>s_{c}:=d / 2+1$. The borderline case $s=s_{c}$ was a folklore open problem. In this paper we consider the physical dimensions $d=2,3$ and show that if we perturb any given smooth initial data in $H^{s_{c}}$ norm, then the corresponding solution can have infinite $H^{s_{c}}$ norm instantaneously at $t>0$. The constructed solutions are unique and even $C^{\infty}$-smooth in some cases. To prove these results we introduce a new strategy: large Lagrangian deformation induces critical norm inflation. As an application we also settle several closely related open problems.
\end{abstract}

\section{INTRODUCTION}

The $d$-dimensional incompressible Euler equation takes the form

$$
\left\{\begin{array}{l}
\partial_{t} u+(u \cdot \nabla) u+\nabla p=0, \quad(t, x) \in \mathbb{R} \times \mathbb{R}^{d}, \\
\nabla \cdot u=0 \\
\left.u\right|_{t=0}=u_{0},
\end{array}\right.
$$

where $u=u(t, x)=\left(u_{1}(t, x), \cdots, u_{d}(t, x)\right): \mathbb{R} \times \mathbb{R}^{d} \rightarrow \mathbb{R}^{d}$ denotes the velocity of the fluid and $p=p(t, x): \mathbb{R} \times \mathbb{R}^{d} \rightarrow \mathbb{R}$ is the pressure. The second equation $\nabla \cdot u=0$ in (1.1) is usually called the incompressibility (divergence-free) condition. By taking the divergence on both sides of the first equation in (1.1), one can recover the pressure from the quadratic term in velocity by inverting the Laplacian in suitable functional spaces. Another way to eliminate the pressure is to use the vorticity formulation. For this we will discuss separately the 2D and 3D case. In $2 \mathrm{D}$, introduce the scalar-valued vorticity function

$$
\omega=-\partial_{2} u_{1}+\partial_{1} u_{2}=\nabla^{\perp} \cdot u, \quad \nabla^{\perp}:=\left(-\partial_{2}, \partial_{1}\right) .
$$

By taking $\nabla^{\perp}$. on both sides of (1.1), we have the equation

$$
\left\{\begin{array}{l}
\partial_{t} \omega+u \cdot \nabla \omega=0, \quad(t, x) \in \mathbb{R} \times \mathbb{R}^{2} \\
u=\nabla^{\perp} \psi=\left(-\partial_{2} \psi, \partial_{1} \psi\right), \quad \Delta \psi=\omega \\
\left.\omega\right|_{t=0}=\omega_{0} .
\end{array}\right.
$$

Under some suitable regularity assumptions, the second equations in (1.2) can be written as a single equation

$$
u=\Delta^{-1} \nabla^{\perp} \omega
$$


which is the usual Biot-Savart law. Alternatively one can express (1.3) as a convolution integral

$$
u=K * \omega, \quad K(x)=\frac{1}{2 \pi} \cdot \frac{x^{\perp}}{|x|^{2}} .
$$

We can then rewrite (1.2) more compactly as

$$
\left\{\begin{array}{l}
\partial_{t} \omega+\left(\Delta^{-1} \nabla^{\perp} \omega \cdot \nabla\right) \omega=0 \\
\left.\omega\right|_{t=0}=\omega_{0} .
\end{array}\right.
$$

We shall frequently refer to (1.4) as the usual 2D Euler equation in vorticity formulation. Note that (1.4) is a transport equation which preserves all $L^{p}, 1 \leq p \leq \infty$ norm of the vorticity $\omega$. In the $3 \mathrm{D}$ case the vorticity is vector-valued and given by

$$
\omega=\operatorname{curl} u=\nabla \times u .
$$

The 3D Euler equation in vorticity formulation has the form

$$
\left\{\begin{array}{l}
\partial_{t} \omega+(u \cdot \nabla) \omega=(\omega \cdot \nabla) u, \\
u=-\Delta^{-1} \nabla \times \omega, \\
\left.\omega\right|_{t=0}=\omega_{0} .
\end{array}\right.
$$

Note that the second equation above is just the Biot-Savart law in 3D. The expression $(\omega \cdot \nabla) u$ is often referred to as the vorticity stretching term. It is one of the main source of difficulties in the wellposedness theory of 3D Euler.

There is by now an extensive literature on the wellposedness theory for Euler equations. We shall only mention a few and refer to Majda-Bertozzi [21] and Constantin [7] for more extensive references. The papers of Lichtenstein [18] and Gunther [12] started the subject of local wellposedness in Hölder spaces $C^{k, \alpha}(k \geq 1$, $0<\alpha<1$ ). In 29] Wolibner obtained global solvability of classical (belonging to Hölder class) solutions for 2D Euler (see Chemin [5] for a modern exposition). In [10] Ebin and Marsden proved the short time existence, uniqueness, regularity, and continuous dependence on initial conditions for solutions of the Euler equation on general compact manifolds (possibly with $C^{\infty}$ boundary). Their method is to topologize the space of diffeomorphisms by Sobolev $H^{s}, s>d / 2+1$ norms and then solve the geodesic equation using contractions. In 4 Bourguignon and Brezis generalized $H^{s}$ to the case of $W^{s, p}$ for $s>d / p+1$. In 15] Kato proved local wellposedness of $d$-dimensional Euler in $C_{t}^{0} H_{x}^{m}$ for initial velocity $u_{0} \in H^{m}\left(\mathbb{R}^{d}\right)$ with integer $m>d / 2+1$. Later Kato and Ponce [17. proved wellposedness in the general Sobolev space $W^{s, p}\left(\mathbb{R}^{d}\right)=(1-\Delta)^{-s / 2} L^{p}\left(\mathbb{R}^{d}\right)$ with real $s>d / p+1$ and $1<p<\infty$. The key argument in [17] is the following commutator estimate for the operator $J^{s}=(1-\Delta)^{s / 2}$ :

$$
\left\|J^{s}(f g)-f J^{s} g\right\|_{p} \lesssim_{d, s, p}\|D f\|_{\infty}\left\|J^{s-1} g\right\|_{p}+\left\|J^{s} f\right\|_{p}\|g\|_{\infty}, \quad 1<p<\infty, s \geq 0 .
$$

To extend the local solutions globally in time, one can use the Beale-Kato-Majda criterion [3] which asserts that (here $s>d / 2+1$ )

$$
\limsup _{t \rightarrow T^{*}}\|u(t, \cdot)\|_{H^{s}\left(\mathbb{R}^{d}\right)}=+\infty,
$$


if and only if

$$
\limsup _{t \rightarrow T^{*}} \int_{0}^{t}\|\omega(s, \cdot)\|_{L^{\infty}\left(\mathbb{R}^{d}\right)} d s=+\infty .
$$

By using this criterion and conservation of $\|\omega\|_{\infty}$ in $2 \mathrm{D}$, one can immediately deduce the global existence of Kato's solutions in dimension two. In [27] (see also [28]) Vishik considered the borderline case $s=d / p+1$ and obtained global solvability for the 2D Euler in Besov space $B_{p, 1}^{2 / p+1}$ with $1<p<\infty$. In [6] Chae proved local existence and uniqueness of solutions to $d$-dimensional Euler in critical Besov space (for velocity) $B_{p, 1}^{d / p+1}\left(\mathbb{R}^{d}\right)$ with $1<p<\infty$. The local wellposedness in $B_{\infty, 1}^{1}\left(\mathbb{R}^{d}\right)$, $d \geq 2$ was settled by Pak and Park in 23 . Roughly speaking, all the aforementioned local wellposedness results rely on finding a certain Banach space $X$ with the norm $\|\cdot\|_{X}$ such that (take $f=\nabla \times u$ and $X=B_{p, 1}^{d / p}$ for example)

(1) If $f \in X$, then $\|f\|_{L^{\infty}}+\left\|\mathcal{R}_{i j} f\right\|_{L^{\infty}} \lesssim\|f\|_{X}$ ( $\mathcal{R}_{i j}$ is the Riesz transform);

(2) Some version of a commutator estimate similar to (1.5) holds in $X$.

The above are essentially minimal conditions needed to close the energy estimates. On the other hand, this type of scheme completely breaks down for the natural borderline Sobolev spaces such as $H^{d / 2+1}$ (in terms of vorticity we have $X=H^{d / 2}$ ) since both conditions will be violated. In [25], Takada constructed] several counterexamples of Kato-Ponce-type commutator estimates in critical Besov $B_{p, q}^{d / p+1}\left(\mathbb{R}^{d}\right)$ and Triebel-Lizorkin spaces $F_{p, q}^{d / p+1}\left(\mathbb{R}^{d}\right)$ for various exponents $p$ and $q$ (For Besov: $1 \leq p \leq \infty, 1<q \leq \infty$; For Triebel-Lizorkin: $1<p<\infty, 1 \leq q \leq \infty$ or $p=q=\infty)$. It should be noted that the vector fields used in his counterexamples are divergence-free. In light of these considerations, a well-known long standing open problem was the following

Conjecture 1.1. The Euler equation (1.1) is illposed for a class of initial data in $H^{d / 2+1}\left(\mathbb{R}^{d}\right)$.

Of course one can state analogous versions of Conjecture 1.1 in similar Sobolev spaces $W^{d / p+1, p}$ or other Besov or Triebel-Lizorkin type spaces with various boundary conditions. A rather delicate matter is to give a precise (and satisfactory) formulation of the illposedness statement in Conjecture 1.1. The formulation and the proof of such a statement requires a deep understanding of how the critical space topology changes under the Euler dynamics.

To begin, one can consider explicit solutions to (1.1). In [11, DiPerna and Majda introduced the following shear flow (in their study of measure-valued solutions for 3D Euler):

$$
u(t, x)=\left(f\left(x_{2}\right), 0, g\left(x_{1}-t f\left(x_{2}\right)\right)\right), \quad x=\left(x_{1}, x_{2}, x_{3}\right),
$$

where $f$ and $g$ are given single variable functions. This explicit flow (sometimes called " $2+1 / 2$ "-dimensional flow) solves (1.1) with pressure $p=0$. DiPerna and Lions used the above flow (see e.g. p152 of [20]) to show that for every $1 \leq p<\infty$, $T>0, M>0$, there exists a smooth shear flow for which $\|u(0)\|_{W^{1, p}\left(\mathbb{T}^{3}\right)}=1$ and $\|u(T)\|_{W^{1, p}\left(\mathbb{T}^{3}\right)}>M$. Recently Bardos and Titi 2] revisited this example and constructed a weak solution which initially lies in $C^{\alpha}$ but does not belong to any $C^{\beta}$ for any $t>0$ and $1>\beta>\alpha^{2}$. By similar arguments one can also deduce

\footnotetext{
${ }^{1}$ Counterexamples for the case $s<d / p+1$ was also considered therein.
} 
illposedness in $F_{\infty, 2}^{1}$ and $B_{\infty, \infty}^{1}$ (see Remark 1 therein). In [22], Misiołek and Yoneda considered the logarithmic Lipschitz space $\mathrm{LL}_{\alpha}\left(\mathbb{R}^{d}\right)$ consisting of continuous functions such that

$$
\|f\|_{\mathrm{LL}_{\alpha}}=\|f\|_{\infty}+\sup _{0<|x-y|<\frac{1}{2}} \frac{|f(x)-f(y)|}{|x-y||\log | x-\left.y\right|^{\alpha}}<\infty .
$$

They used the above shear-flow example to generate illposedness of 3D Euler in $\mathrm{LL}_{\alpha}$ for any $0<\alpha \leq 1$. In connection with Conjecture 1.1, a related issue is the dependence of the solution operator on the underlying topology. In [16, to describe the sharpness of the continuous dependence on initial data in his wellposedness result, Kato showed that (see Example 5.2 therein) the solution operator for the Burgers equation is not Hölder continuous in $H^{s}(\mathbb{R}), s \geq 2$ norm for any prescribed Hölder exponent. In [13] Himonas and Misiołek proved that for the Euler equation the data-to-solution map is not uniform continuous in $H^{s}(\Omega)$ topology where $s \in \mathbb{R}$ if $\Omega=\mathbb{T}^{d}=\mathbb{R}^{d} / 2 \pi \mathbb{Z}^{d}$ and $s>0$ if $\Omega=\mathbb{R}^{d}$. Very recently Inci 14 strengthened this result and showed for any $T>0$ that the solution map $u(0) \rightarrow u(T)$ is nowhere locally uniformly continuous for $H^{s}\left(\mathbb{R}^{n}\right), s>n / 2+1$. In [8], Cheskidov and Shvydkoy proved illposedness of $d$-dimensional Euler in Besov spaces $B_{r, \infty}^{s}\left(\mathbb{T}^{d}\right)$ where $s>0$ if $r>2$ and $s>d\left(\frac{2}{r}-1\right)$ if $1 \leq r \leq 2$. However, as was pointed out by the aforementioned authors, the above works do not address the borderline Sobolev space $H^{d / 2+1}$ or similar critical spaces which was an outstanding open problem.

The purpose of this work is to completely settle the borderline case $H^{d / 2+1}$ (Conjecture 1.1) and several other related open problems. Roughly speaking, we prove the following

Theorem. Let the dimension $d=2,3$. The Euler equation (1.1) is illposed in the Sobolev space $W^{d / p+1, p}$ for any $1<p<\infty$ or the Besov space $B_{p, q}^{d / p+1}$ for any $1<p<\infty, 1<q \leq \infty$.

As a matter of fact, we shall show that in the borderline case, ill-posedness holds in the strongest sense. Namely for any given smooth initial data, we shall find special perturbations which can be made arbitrarily small in the critical Sobolev norm, such that the corresponding perturbed solution is unique (in other functional spaces) but loses borderline Sobolev regularity instantaneously in time. Our analysis shows that in some sense the illposedness happens in a very generic way. In particular, it is "dense" in the $H^{d / 2+1}$ (and similarly for other critical spaces) topology.

We now state more precisely the main results. The first result is for $2 \mathrm{D}$ Euler with non-compactly supported data. A special feature is that our constructed solutions are $C^{\infty}$-smooth which are classical solutions.

Theorem 1.2 (2D non-compact case). For any given $\omega_{0}^{(g)} \in C_{c}^{\infty}\left(\mathbb{R}^{2}\right) \cap \dot{H}^{-1}\left(\mathbb{R}^{2}\right)$ and any $\epsilon>0$, we can find a $C^{\infty}$ perturbation $\omega_{0}^{(p)}: \mathbb{R}^{2} \rightarrow \mathbb{R}$ such that the following hold true:

(1) $\left\|\omega_{0}^{(p)}\right\|_{\dot{H}^{1}\left(\mathbb{R}^{2}\right)}+\left\|\omega_{0}^{(p)}\right\|_{L^{1}\left(\mathbb{R}^{2}\right)}+\left\|\omega_{0}^{(p)}\right\|_{L^{\infty}\left(\mathbb{R}^{2}\right)}+\left\|\omega_{0}^{(p)}\right\|_{\dot{H}^{-1}\left(\mathbb{R}^{2}\right)}<\epsilon$.

(2) Let $\omega_{0}=\omega_{0}^{(g)}+\omega_{0}^{(p)}$. The initial velocity $u_{0}=\Delta^{-1} \nabla^{\perp} \omega_{0}$ has regularity $u_{0} \in H^{2}\left(\mathbb{R}^{2}\right) \cap C^{\infty}\left(\mathbb{R}^{2}\right) \cap L^{\infty}\left(\mathbb{R}^{2}\right)$. 
(3) There exists a unique classical solution $\omega=\omega(t)$ to the $2 D$ Euler equation (in vorticity form)

$$
\left\{\begin{array}{l}
\partial_{t} \omega+\left(\Delta^{-1} \nabla^{\perp} \omega \cdot \nabla\right) \omega=0, \quad 0<t \leq 1, x \in \mathbb{R}^{2}, \\
\left.\omega\right|_{t=0}=\omega_{0},
\end{array}\right.
$$

satisfying

$$
\max _{0 \leq t \leq 1}\left(\|\omega(t, \cdot)\|_{L^{1}}+\|\omega(t, \cdot)\|_{L^{\infty}}+\|\omega(t, \cdot)\|_{\dot{H}^{-1}}\right)<\infty .
$$

Here $\omega(t) \in C^{\infty}, u(t)=\Delta^{-1} \nabla^{\perp} \omega(t) \in C^{\infty} \cap L^{2} \cap L^{\infty}$ for each $0 \leq t \leq 1$.

(4) For any $0<t_{0} \leq 1$, we have

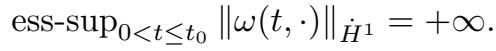

Remark 1.3. The $\dot{H}^{-1}$ assumption on the vorticity data $\omega_{0}^{(g)}$ can be removed. We include it here simply to stress that the perturbed solution can inherit $\dot{H}^{-1}$ regularity which is natural since the corresponding velocity will be in $L^{2}$. Of course one can also state similar results for $\omega_{0}^{(g)} \in H^{s}$ with $s>1$ or some other subcritical functional spaces.

Remark 1.4. In our construction, although the initial velocity $u_{0}$ is $C^{\infty}$-smooth, its gradient turns out to be unbounded, i.e. $\left\|\nabla u_{0}\right\|_{L^{\infty}\left(\mathbb{R}^{2}\right)}=+\infty$.

Remark 1.5. In [16] Kato introduced the uniformly local Sobolev spaces $L_{u l}^{p}\left(\mathbb{R}^{d}\right)$ (see (2.3) $)$ and $H_{u l}^{s}\left(\mathbb{R}^{d}\right)$. These spaces contain $H^{s}\left(\mathbb{R}^{d}\right)$ and the periodic space $H^{s}\left(\mathbb{T}^{d}\right)$. The statement (1.6) in Theorem 1.2 can be improved to

$$
\operatorname{ess-sup}_{0<t \leq t_{0}}\|\nabla \omega(t, \cdot)\|_{L_{u l}^{2}\left(\mathbb{R}^{2}\right)}=+\infty .
$$

Similar results also hold for Theorem 1.61 .10 below. We shall not state them but leave it to interested readers.

Our next result is for the compactly supported data for the 2D Euler equation. Note that this result carries over (with simple changes) to the periodic case as well. For simplicity we shall consider vorticity functions having one-fold symmetry. For example, we shall say $g=g\left(x_{1}, x_{2}\right): \mathbb{R}^{2} \rightarrow \mathbb{R}$ is odd in $x_{1}$ if

$$
g\left(-x_{1}, x_{2}\right)=-g\left(x_{1}, x_{2}\right), \quad \forall x=\left(x_{1}, x_{2}\right) \in \mathbb{R}^{2} .
$$

It is not difficult to check that the one-fold odd symmetry (of the vorticity function) is preserved by the Euler flow.

Theorem 1.6 (2D compact case). Let $\omega_{0}^{(g)} \in C_{c}^{\infty}\left(\mathbb{R}^{2}\right) \cap \dot{H}^{-1}\left(\mathbb{R}^{2}\right)$ be any given vorticity function which is odd in $x_{2} 2$ For any such $\omega_{0}^{(g)}$ and any $\epsilon>0$, we can find a perturbation $\omega_{0}^{(p)}: \mathbb{R}^{2} \rightarrow \mathbb{R}$ such that the following hold true:

(1) $\omega_{0}^{(p)}$ is compactly supported (in a ball of radius $\leq 1$ ), continuous and

$$
\left\|\omega_{0}^{(p)}\right\|_{\dot{H}^{1}\left(\mathbb{R}^{2}\right)}+\left\|\omega_{0}^{(p)}\right\|_{L^{\infty}\left(\mathbb{R}^{2}\right)}+\left\|\omega_{0}^{(p)}\right\|_{\dot{H}^{-1}\left(\mathbb{R}^{2}\right)}<\epsilon .
$$

\footnotetext{
${ }^{2}$ Similar results also hold for vorticity functions which are odd in $x_{1}$, or odd in both $x_{1}$ and $x_{2}$.
} 
(2) Let $\omega_{0}=\omega_{0}^{(g)}+\omega_{0}^{(p)}$. Corresponding to $\omega_{0}$ there exists a unique timeglobal solution $\omega=\omega(t)$ to the Euler equation satisfying $\omega(t) \in L^{\infty} \cap \dot{H}^{-1}$. Furthermore $\omega \in C_{t}^{0} C_{x}^{0}$ and $u=\Delta^{-1} \nabla^{\perp} \omega \in C_{t}^{0} L_{x}^{2} \cap C_{t}^{0} C_{x}^{\alpha}$ for any $0<$ $\alpha<1$.

(3) $\omega(t)$ has additional local regularity in the following sense: there exists $x_{*} \in$ $\mathbb{R}^{2}$ such that for any $x \neq x_{*}$, there exists a neighborhood $N_{x} \ni x, t_{x}>0$ such that $w(t, \cdot) \in C^{\infty}\left(N_{x}\right)$ for any $0 \leq t \leq t_{x}$.

(4) For any $0<t_{0} \leq 1$, we have

$$
\operatorname{ess}^{-\sup _{0<t \leq t_{0}}}\|\omega(t, \cdot)\|_{\dot{H}^{1}}=+\infty .
$$

More precisely, there exist $0<t_{n}^{1}<t_{n}^{2}<\frac{1}{n}$, open precompact sets $\Omega_{n}$, $n=1,2,3, \cdots$ such that $\omega(t) \in C^{\infty}\left(\Omega_{n}\right)$ for all $0 \leq t \leq t_{n}^{2}$, and

$$
\|\nabla \omega(t, \cdot)\|_{L^{2}\left(\Omega_{n}\right)}>n, \quad \forall t \in\left[t_{n}^{1}, t_{n}^{2}\right] .
$$

Remark 1.7. In 30] Yudovich proved the existence and uniqueness of weak solutions to $2 \mathrm{D}$ Euler in bounded domains for $L^{\infty}$ vorticity data. The uniqueness result (for bounded domain in general dimensions $d \geq 2$ ) was improved in [31] allowing vorticty $\omega \in \cap_{p_{0} \leq p<\infty} L^{p}$ and $\|\omega\|_{p} \leq C \theta(p)$ with $\theta(p)$ growing relatively slowly in $p$ (such as $\theta(p)=\log p$ ). Vishik [28] proved the uniqueness of weak solutions to Euler in $\mathbb{R}^{d}, d \geq 2$, under the following assumptions:

- $\omega \in L^{p_{0}}, 1<p_{0}<d$,

- For some $a(k)>0$ with the property

$$
\int_{1}^{\infty} \frac{1}{a(k)} d k=+\infty
$$

it holds that

$$
\left|\sum_{j=2}^{k}\left\|P_{2^{j}} \omega\right\|_{\infty}\right| \leq \text { const } \cdot a(k), \quad \forall k \geq 4 .
$$

In other words uniqueness is guaranteed as long as $\omega$ has a little bit integrability and the partial sum of the Besov $\dot{B}_{\infty, 1}^{0}$ norm of $\omega$ is allowed to diverge in a controlled fashion. Since we have uniform in time $L^{\infty}$ control of the vorticity $\omega$ in both $2 \mathrm{D}$ and 3D (see Theorem 1.8 1.10 below), the uniqueness of the constructed solution is not an issue and we shall not discuss this point further in this work.

Our third result is for 3D Euler with non-compactly supported data. As is wellknown the lifespan of solutions to 3D Euler emanating from smooth initial data is an outstanding open problem. Since we are perturbing smooth initial data using functions with critical Sobolev regularity, we need to make sure the perturbed solution has a positive lifespan in some suitable functional spaces. In the noncompact data case, this issue turns out to be immaterial since we can choose the patches sufficiently far away from each other and the lifespan of each patch is well under control.

\footnotetext{
${ }^{3}$ Actually it is easy to show that $u$ is log-Lipschitz.
} 
Theorem 1.8 (3D non-compact case). Consider the 3D incompressible Euler equation in vorticity form:

$$
\left\{\begin{array}{l}
\partial_{t} \omega+(u \cdot \nabla) \omega=(\omega \cdot \nabla) u, \quad t>0, x=\left(x_{1}, x_{2}, z\right) \in \mathbb{R}^{3} ; \\
u=-\Delta^{-1} \nabla \times \omega, \\
\left.\omega\right|_{t=0}=\omega_{0} .
\end{array}\right.
$$

For any given $\omega_{0}^{(g)} \in C_{c}^{\infty}\left(\mathbb{R}^{3}\right)$ and any $\epsilon>0$, we can find a $T_{0}=T_{0}\left(\omega_{0}^{(g)}\right)>0$ and $C^{\infty}$ perturbation $\omega_{0}^{(p)}: \mathbb{R}^{3} \rightarrow \mathbb{R}^{3}$ such that the following hold true:

(1) $\left\|\omega_{0}^{(p)}\right\|_{\dot{H}^{\frac{3}{2}\left(\mathbb{R}^{3}\right)}}+\left\|\omega_{0}^{(p)}\right\|_{L^{1}\left(\mathbb{R}^{3}\right)}+\left\|\omega_{0}^{(p)}\right\|_{L^{\infty}\left(\mathbb{R}^{3}\right)}<\epsilon$.

(2) Let $\omega_{0}=\omega_{0}^{(g)}+\omega_{0}^{(p)}$. Let $u_{0}$ be the velocity corresponding to the initial vorticity $\omega_{0}$. We have $u_{0} \in H^{\frac{5}{2}}\left(\mathbb{R}^{3}\right) \cap C^{\infty}\left(\mathbb{R}^{3}\right) \cap L^{\infty}\left(\mathbb{R}^{3}\right)$.

(3) Corresponding to $\omega_{0}$, there exists a unique solution $\omega=\omega(t)$ to (1.7) on the whole time interval $\left[0, T_{0}\right]$ such that

$$
\sup _{0 \leq t \leq 1}\left(\|\omega(t, \cdot)\|_{L^{1}}+\|\omega(t, \cdot)\|_{L^{\infty}}\right)<\infty .
$$

Moreover $\omega \in C^{\infty}$ and $u \in C^{\infty}$ so that the solution is actually classical.

(4) For any $0<t_{0} \leq T_{0}$, we have

$$
\operatorname{ess} \sup _{0<t \leq t_{0}}\|\omega(t, \cdot)\|_{\dot{H}^{\frac{3}{2}}}=+\infty .
$$

Remark 1.9. If the vorticity $\omega_{0}^{(g)}$ is axisymmetric (see (1.8) below), then we can choose $T_{0}>0$ to be any positive number. This is due to the fact that for 3D Euler smooth axisymmetric flows without swirl exist globally in time.

The following theorem concerns the 3D Euler case with compactly supported initial vorticity. In this case the situation is more complicated than that in Theorem 1.8. For convenience we will work with a class of axisymmetric vorticity functions $\omega$ having the form:

$$
\omega(x)=\omega^{\theta}(r, z) e_{\theta}, \quad x=\left(x_{1}, x_{2}, z\right), r=\sqrt{x_{1}^{2}+x_{2}^{2}},
$$

where $\omega^{\theta}$ is scalar-valued and $e_{\theta}=\frac{1}{r}\left(-x_{2}, x_{1}, 0\right)$. The corresponding velocity fields are usually called axisymmetric without swirl flows. In this paper we shall call such $\omega$ axisymmetric without swirl vorticity or simply axisymmetric vorticity when there is no obvious confusion. The theory of axisymmetric flows on $\mathbb{R}^{3}$ and some recent developments are reviewed in the beginning of Section 7 .

Theorem 1.10 (3D compact case). For any given axisymmetric vorticity $\omega_{0}^{(g)} \in$ $C_{c}^{\infty}\left(\mathbb{R}^{3}\right)$ and any $\epsilon>0$, we can find a perturbation $\omega_{0}^{(p)}: \mathbb{R}^{3} \rightarrow \mathbb{R}^{3}$ such that the following hold true:

(1) $\omega_{0}^{(p)}$ is compactly supported (in a ball of radius $\leq 1$ ), continuous and

$$
\left\|\omega_{0}^{(p)}\right\|_{\dot{H}^{\frac{3}{2}\left(\mathbb{R}^{3}\right)}}+\left\|\omega_{0}^{(p)}\right\|_{L^{\infty}\left(\mathbb{R}^{3}\right)}<\epsilon .
$$

(2) Let $\omega_{0}=\omega_{0}^{(g)}+\omega_{0}^{(p)}$. Corresponding to $\omega_{0}$ there exists a unique solution $\omega=\omega(t, x)$ to the Euler equation (1.7) on the time interval $[0,1]$ satisfying

$$
\begin{aligned}
& \sup _{0 \leq t \leq 1}\|\omega(t, \cdot)\|_{L^{\infty}}<\infty, \\
& \operatorname{supp}(\omega(t, \cdot)) \subset\{x,|x|<R\}, \quad \forall 0 \leq t \leq 1,
\end{aligned}
$$


where $R>0$ is some constant. Furthermore $\omega \in C_{t}^{0} C_{x}^{0}$ and $u \in C_{t}^{0} L_{x}^{2} \cap$ $C_{t}^{0} C_{x}^{\alpha}$ for any $\alpha<1$.

(3) $\omega(t)$ has additional local regularity in the following sense: there exists $x_{*} \in$ $\mathbb{R}^{3}$ such that for any $x \neq x_{*}$, there exists a neighborhood $N_{x} \ni x, t_{x}>0$ such that $w(t) \in C^{\infty}\left(N_{x}\right)$ for any $0 \leq t \leq t_{x}$.

(4) For any $0<t_{0} \leq 1$, we have

$$
\operatorname{ess}^{-\sup _{0<t \leq t_{0}}}\|\omega(t, \cdot)\|_{\dot{H}^{\frac{3}{2}}\left(\mathbb{R}^{3}\right)}=+\infty .
$$

More precisely, there exist $0<t_{n}^{1}<t_{n}^{2}<\frac{1}{n}$, open precompact sets $\Omega_{n}^{1}, \Omega_{n}^{2}$ with $\Omega_{n}^{1} \subset \overline{\Omega_{n}^{1}} \subset \Omega_{n}^{2}, n=1,2,3, \cdots$ such that

- $\omega(t) \in C^{\infty}\left(\Omega_{n}^{2}\right)$ for all $0 \leq t \leq t_{n}^{2}$;

- $\omega(t, x) \equiv 0$ for any $x \in \Omega_{n}^{2} \backslash \Omega_{n}^{1}, 0 \leq t \leq t_{n}^{2}$.

- Define $\omega_{n}(t, x)=\omega(t, x)$ for $x \in \Omega_{n}^{1}$, and $\omega_{n}(t, x)=0$ otherwise. Then $\omega_{n} \in C_{c}^{\infty}\left(\mathbb{R}^{3}\right)$,

$$
\left\|\omega_{n}(t, \cdot)\right\|_{\dot{H}^{\frac{3}{2}\left(\mathbb{R}^{3}\right)}}>n, \quad \forall t_{n}^{1} \leq t \leq t_{n}^{2} .
$$

and

$$
\left\|\left(|\nabla|^{3} \omega_{n}\right)(t, \cdot)\right\|_{L^{2}\left(x \in \mathbb{R}^{3} \backslash \Omega_{n}^{2}\right)} \leq 1, \quad \forall 0 \leq t \leq t_{n}^{2} .
$$

Remark 1.11. We stress that the situation here in Theorem 1.10 is much more complex than the 2D case in Theorem 1.6. Due to the nonlocal character of the fractional differentiation operator $|\nabla|^{\frac{3}{2}}$, we have to include the additional constraint (1.12) in our construction in order to derive (1.10) from (1.11). We briefly sketch the argument as follows. Suppose $\|\omega(\tau, \cdot)\|_{\dot{H}^{\frac{3}{2}}}<\infty$, for some $\tau \in\left[t_{n}^{1}, t_{n}^{2}\right]$. Then we write

$$
\omega(\tau)=\omega_{n}(\tau)+g_{n}(\tau),
$$

where $g_{n}(\tau)=\omega(\tau)-\omega_{n}(\tau)$ also has finite $\dot{H}^{\frac{3}{2}}$-norm. Clearly

$$
\begin{aligned}
\|\omega(\tau)\|_{\dot{H}^{\frac{3}{2}}}^{2} & =\left\|\omega_{n}(\tau)\right\|_{\dot{H}^{\frac{3}{2}}}^{2}+\left\|g_{n}(\tau)\right\|_{\dot{H}^{\frac{3}{2}}}^{2} \\
& +2\left\langle|\nabla|^{\frac{3}{2}} \omega_{n}(\tau),|\nabla|^{\frac{3}{2}} g_{n}(\tau)\right\rangle,
\end{aligned}
$$

where $\langle$,$\rangle denotes the usual L^{2}$ inner product on $L^{2}\left(\mathbb{R}^{3}\right)$. Now observe that $\operatorname{supp}\left(g_{n}(\tau)\right) \subset$ $\mathbb{R}^{3} \backslash \Omega_{n}^{2}$ and $\left\|g_{n}(\tau)\right\|_{L^{2}} \lesssim\|\omega(\tau)\|_{2}\left(g_{n}(\tau)\right.$ and $\omega_{n}(\tau)$ have disjoint supports), therefore

$$
\begin{aligned}
& \left|\left\langle|\nabla|^{\frac{3}{2}} \omega_{n}(\tau),|\nabla|^{\frac{3}{2}} g_{n}(\tau)\right\rangle\right| \\
= & \left|\left\langle|\nabla|^{3} \omega_{n}(\tau), g_{n}(\tau)\right\rangle\right| \\
\leq & \left\|\left(|\nabla|^{3} \omega_{n}\right)(\tau)\right\|_{L^{2}\left(\mathbb{R}^{3} \backslash \Omega_{n}^{2}\right)}\left\|g_{n}(\tau)\right\|_{L^{2}} \\
\lesssim & \|\omega(\tau)\|_{2} \lesssim 1 .
\end{aligned}
$$

Hence for $n$ sufficiently large, we have for any $\tau \in\left[t_{n}^{1}, t_{n}^{2}\right]$, either

$$
\|\omega(\tau)\|_{\dot{H}^{\frac{3}{2}}}=+\infty
$$

or

$$
\|\omega(\tau)\|_{\dot{H}^{\frac{3}{2}}}>\frac{n}{2}
$$

This obviously implies (1.10). 
Theorem 1.2 1.10 can be sharpened significantly. We have the following Besov version which essentially includes all previous theorems as special cases. In order not to overburden with notations, we shall state an informal version. The detailed (and more precise) statements can be found in Section 9 and Theorem 9.1 9.4 therein.

Theorem 1.12 (Besov case). Let $d=2,3$. For any smooth initial velocity $u_{0}^{(g)}$, any $\epsilon>0$, and any $1<p<\infty, 1<q \leq \infty$, there exist a nearby initial velocity $u_{0} \in B_{p, q}^{\frac{d}{p}+1}$ such that $\left\|u_{0}-u_{0}^{(g)}\right\|_{B_{p, q}^{\frac{d}{p}}}<\epsilon$, and the corresponding solution satisfies

$$
\operatorname{ess}^{-\sup _{0<t<t_{0}}}\|u(t, \cdot)\|_{\dot{B}_{p, \infty}^{\frac{d}{p}+1}}=+\infty
$$

for any $t_{0}>0$.

Our last result concerns the illposedness in the usual Sobolev $W^{s, p}$ spaces.

Theorem 1.13. Let $d=2,3$. For any smooth initial velocity $u_{0}^{(g)}$, any $\epsilon>0$, and any $1<p<\infty$, there exists a nearby initial velocity $u_{0} \in W^{d / p+1, p}$ such that $\left\|u_{0}-u_{0}^{(g)}\right\|_{W^{d / p+1, p}}<\epsilon$, and the solution corresponding to $u_{0}$ satisfies

$$
\operatorname{ess-Sup}_{0<t<t_{0}}\|u(t, \cdot)\|_{\dot{W}^{d / p+1, p}}=+\infty
$$

for any $t_{0}>0$.

The proof of Theorem 1.13 will be omitted. It can be subsumed under a more general argument dealing with Triebel-Lizorkin spaces which we will address in a forthcoming paper.

In the rest of this introduction, we give a brief overview of the proofs of Theorem 1.2 1.12. The overall scheme consists of three steps. The first two steps are devoted to local constructions. The last step is a global patching argument. We shall explain the main arguments for the $2 \mathrm{D}$ Euler case with $H^{2}$ (for vorticity the space is $H^{1}$ ) as the working critical space. Some additional technical points needed to treat the $3 \mathrm{D}$ case will be clarified along the way.

Step 1. Creation of large Lagrangian deformation. Define the flow map associated to (1.1) as $\phi=\phi(t, x)$ which solves

$$
\left\{\begin{array}{l}
\partial_{t} \phi(t, x)=u(t, \phi(t, x)), \\
\phi(0, x)=x
\end{array}\right.
$$

For any $0<T \ll 1, B\left(x_{0}, \delta\right) \subset \mathbb{R}^{2}$ with $x_{0} \in \mathbb{R}^{2}$ arbitrary and $\delta \ll 1$, we choose initial (vorticity) data $\omega_{a}^{(0)}$ such that

$$
\left\|\omega_{a}^{(0)}\right\|_{L^{1}}+\left\|\omega_{a}^{(0)}\right\|_{L^{\infty}}+\left\|\omega_{a}^{(0)}\right\|_{H^{1}} \ll 1,
$$

and

$$
\sup _{0<t \leq T}\left\|D \phi_{a}(t, \cdot)\right\|_{\infty} \gg 1
$$

Here $\phi_{a}$ is the flow map associated with the velocity $u=u_{a}$ which solves (1.1) with $\omega_{a}^{(0)}$ as vorticity initial data. By translation invariance of Euler it suffices to consider the case $x_{0}=0$. In our construction we restrict to some special flows which have odd symmetry and admit the origin as a stagnation point. We prove that the deformation matrix $D u$ remains essentially 
hyperbolic near the spatial origin in the short time interval considered (cf. Proposition 3.5 and Proposition 3.6).

Step 2. Local inflation of critical norm. As was already mentioned, the critical norm for the vorticity is $H^{1}$. The solution constructed in Step 1 does not necessarily obey $\sup _{0<t \leq T}\left\|\nabla \omega_{a}(t)\right\|_{2} \gg 1$. We then perturb the initial data $\omega_{a}^{(0)}$ and take

$$
\omega_{b}^{(0)}=\omega_{a}^{(0)}+\frac{1}{k} \sin (k f(x)) g(x),
$$

where $k$ is a very large parameter. The function $g$ is smooth and has $o(1)$ $L^{2}$ norm The function $f(x)$ and the support of $g$ will be chosen depending on the exact location of the maximum of $\left\|D \phi_{a}(t, \cdot)\right\|_{\infty}$. Of course since the initial data is altered, the corresponding characteristic line (flow map) is changed as well. For this we run a perturbation argument in $W^{1,4}$ so that $\left\|D \phi_{b}(t, \cdot)-D \phi_{a}(t, \cdot)\right\|_{\infty} \ll 1$. The same argument is used to show that in the main order the $H^{1}$ norm of the solution corresponding to $\omega_{b}^{(0)}$ is inflated through the Lagrangian deformation matrix $D \phi_{a}$. The technical details are elaborated in Proposition 4.2 .

Step 3. Gluing of patch solutions. The construction in previous two steps can be repeated in infinitely many small patches which stay away from each other initially. To glue these solutions together we need to differentiate two situations. In the case of Theorem 1.2, we exploit the unboundedness nature of $\mathbb{R}^{2}$ and add each patches sequentially. Each time a new patch is added, we choose the distance between it and the old patches so large such that their interaction is very small. The key properties exploited here are the finite transport speed of the Euler flow and spatial decay of the Riesz kernel. In the case of Theorem [1.6, we need to deal with compactly supported data. This forces us to analyze in detail the interactions of the patches since the patches can become infinitely close to each other. For each $n \geq 2$, define $\omega_{\leq n-1}$ the existing patch and $\omega_{n}$ the current (to be added) patch. It turns out that there exists a patch time $T_{n}$ such that for $0 \leq t \leq T_{n}$, the patch $\omega_{n}$ has disjoint support from $\omega_{\leq n-1}$, and obeys the dynamics

$$
\partial_{t} \omega_{n}+\Delta^{-1} \nabla^{\perp} \omega_{n} \cdot \nabla \omega_{\leq n-1}+\Delta^{-1} \nabla^{\perp} \omega_{n} \cdot \nabla \omega_{n}=0 .
$$

By a suitable re-definition of the patch center and change of variable, we find that $\tilde{\omega}_{n}$ (which is $\omega_{n}$ expressed in the new variable) satisfies the equation

$$
\begin{aligned}
& \partial_{t} \tilde{\omega}_{n}+\Delta^{-1} \nabla^{\perp} \tilde{\omega}_{n} \cdot \nabla \tilde{\omega}_{n} \\
& \quad+b(t)\left(\begin{array}{c}
-y_{1} \\
y_{2}
\end{array}\right) \cdot \nabla \tilde{\omega}_{n}+r(t, y) \cdot \nabla \tilde{\omega}_{n}=0,
\end{aligned}
$$

where $b(t)=O(1)$ and $|r(t, y)| \lesssim|y|^{2}$. We then choose initial data for $\omega_{n}$ such that within patch time $0<t \leq T_{n}$ the critical norm of $\omega_{n}$ inflates rapidly. As we take $n \rightarrow \infty$, the patch time $T_{n} \rightarrow 0$ and $\omega_{n}$ becomes more and more localized. Note that the whole solution (consisting of all patches $\omega_{n}$ ) is actually a time-global solution. During interaction time $T_{n}$ the patch $\omega_{n}$ produces the desired norm inflation since it stays disjoint from all the

\footnotetext{
${ }^{4}$ In the actual perturbation argument, we need to divide by a suitable power of $\|D \phi\|_{\infty}$.
} 
other patches. The details of the perturbation analysis can be found in Lemma 6.4 (and some related lemmas in Section 6).

The 3D case. Compared with the $2 \mathrm{D}$ case, the first difficulty in $3 \mathrm{D}$ is the lack of $L^{p}$ conservation of the vorticity. It is deeply connected with the vorticity stretching term $(\omega \cdot \nabla) u$. To simplify the analysis we take the axisymmetric flow without swirl as the basic building block for the whole construction. The vorticity equation in the axisymmetric case (see the beginning of Section 7) takes the form

$$
\partial_{t}\left(\frac{\omega}{r}\right)+(u \cdot \nabla)\left(\frac{\omega}{r}\right)=0, \quad r=\sqrt{x_{1}^{2}+x_{2}^{2}}, x=\left(x_{1}, x_{2}, z\right) .
$$

Owing to the denominator $r$, the solution formula for $\omega$ then acquires an additional metric factor (compared with 2D) which represents the vorticity stretching effect in the axisymmetric setting. A lot of analysis (cf. Proposition 7.11) goes into controlling the metric factor by the large Lagrangian deformation matrix and producing the desired $H^{3 / 2}$ norm inflation. In our construction the patch solutions which are made of asymmetric without swirl flows typically carry infinite $\|\omega / r\|_{L^{3,1}}$ norm (when summing all the patches together). To glue these solutions together in the $3 \mathrm{D}$ compactly supported case, we need to run a new perturbation argument (cf. Lemma 8.1) which allows to add each new patch $\omega_{n}$ with sufficiently small $\left\|\omega_{n}\right\|_{\infty}$ norm (over the whole lifespan) such that the effect of the large $\left\|\omega_{n} / r\right\|_{L^{3,1}}$ becomes negligible. All in all, the constructed patch solutions converge in the $C^{0}$ metric after building several auxiliary lemmas (cf. Lemma 8.5. Proposition 8.6).

We have roughly described the whole strategy of the proof although some technical points could not be elucidated or even mentioned in this short introduction. In some sense our approach is a hybrid of the Lagrangian point of view and the Eulerian one, using in an essential way several features of the Euler dynamics: finite speed propagation and weak interaction between well-separated vortex patches. The rest of this paper is organized as follows. In Section 2 we set up some basic notations and preliminaries. In Section 3 we describe in detail the first part of the local construction for the $2 \mathrm{D}$ case. Section 4 is devoted to the perturbation argument needed for the 2D local construction step. In Section 5 and 6 we treat the $2 \mathrm{D}$ noncompact case and compactly supported case separately. Section 7-8 are devoted to the construction in the 3D case. Finally in Section 9 we give details for the Besov space case.

Acknowledgements. J. Bourgain was supported in part by NSF No. DMS0808042 and DMS-0835373. D. Li was supported in part by NSF under agreement No. DMS-1128155. Any opinions, findings and conclusions or recommendations expressed in this material are those of the authors and do not necessarily reflect the views of the National Science Foundation. D. Li was also supported by an Nserc discovery grant.

\section{Notation and Preliminaries}

For any two quantities $X$ and $Y$, we denote $X \lesssim Y$ if $X \leq C Y$ for some harmless constant $C>0$. Similarly $X \gtrsim Y$ if $X \geq C Y$ for some $C>0$. We denote $X \sim Y$ if $X \lesssim Y$ and $Y \lesssim X$. We shall write $X \lesssim Z_{1}, Z_{2}, \cdots, Z_{k} \quad Y$ if $X \leq C Y$ and the 
constant $C$ depends on the quantities $\left(Z_{1}, \cdots, Z_{k}\right)$. Similarly we define $\gtrsim_{Z_{1}, \cdots, Z_{k}}$ and $\sim_{Z_{1}, \cdots, Z_{k}}$.

We shall denote by $X+$ any quantity of the form $X+\epsilon$ for any $\epsilon>0$. For example we shall write

$$
Y \lesssim 2^{X+}
$$

if $Y \lesssim_{\epsilon} 2^{X+\epsilon}$ for any $\epsilon>0$. The notation $X-$ is similarly defined.

For any center $x_{0} \in \mathbb{R}^{d}$ and radius $R>0$, we use $B\left(x_{0}, R\right):=\left\{x \in \mathbb{R}^{d}:\left|x-x_{0}\right|<\right.$ $R\}$ to denote the open Euclidean ball. More generally for any set $A \subset \mathbb{R}^{d}$, we denote

$$
B(A, R):=\left\{y \in \mathbb{R}^{d}:|y-x|<R \text { for some } x \in A\right\} .
$$

For any two sets $A_{1}, A_{2} \subset \mathbb{R}^{d}$, we define

$$
d\left(A_{1}, A_{2}\right)=\operatorname{dist}\left(A_{1}, A_{2}\right)=\inf \left\{|x-y|: x \in A_{1}, y \in A_{2}\right\} .
$$

For any $f$ on $\mathbb{R}^{d}$, we denote the Fourier transform of $f$ has

$$
(\mathcal{F} f)(\xi)=\hat{f}(\xi)=\int_{\mathbb{R}^{d}} f(x) e^{-i \xi \cdot x} d x .
$$

The inverse Fourier transform of any $g$ is given by

$$
\left(\mathcal{F}^{-1} g\right)(x)=\frac{1}{(2 \pi)^{d}} \int_{\mathbb{R}^{d}} g(\xi) e^{i x \cdot \xi} d \xi .
$$

For any $1 \leq p \leq \infty$ we use $\|f\|_{p},\|f\|_{L^{p}\left(\mathbb{R}^{d}\right)}$, or $\|f\|_{L_{x}^{p}\left(\mathbb{R}^{d}\right)}$ to denote the usual Lebesgue norm on $\mathbb{R}^{d}$. The Sobolev space $H^{1}\left(\mathbb{R}^{d}\right)$ is defined in the usual way as the completion of $C_{c}^{\infty}$ functions under the norm $\|f\|_{H^{1}}=\|f\|_{2}+\|\nabla f\|_{2}$. For any $s \in \mathbb{R}$, we define the homogeneous Sobolev norm of a tempered distribution $f: \mathbb{R}^{d} \rightarrow \mathbb{R}$ as

$$
\|f\|_{\dot{H}^{s}}=\left(\int_{\mathbb{R}^{d}}|\xi|^{2 s}|\hat{f}(\xi)|^{2} d \xi\right)^{\frac{1}{2}} .
$$

We use the Fourier transform to define the fractional differentiation operators $|\nabla|^{s}$ by the formula

$$
\widehat{|\nabla|^{s} f}(\xi)=|\xi|^{s} \hat{f}(\xi) .
$$

For any integer $n \geq 0$ and any open set $U \subset \mathbb{R}^{d}$, we use the notation $C^{n}(U)$ to denote functions on $U$ whose $n^{\text {th }}$ derivatives are all continuous.

For any $1 \leq p<\infty$, we denote by $L_{u l}^{p}\left(\mathbb{R}^{d}\right)$ the Banach space endowed with the norm

$$
\|u\|_{L_{u l}^{p}\left(\mathbb{R}^{d}\right)}:=\sup _{x \in \mathbb{R}^{d}}\left(\int_{|y-x|<1}|u(y)|^{p} d y\right)^{\frac{1}{p}} .
$$

Let $\phi \in C_{c}^{\infty}\left(\mathbb{R}^{d}\right)$ be not identically zero. The condition $u \in L_{u l}^{p}$ is equivalent to

$$
\sup _{x \in \mathbb{R}^{d}}\|\phi(\cdot-x) u(\cdot)\|_{L^{p}\left(\mathbb{R}^{d}\right)}<\infty .
$$

For any $s \in \mathbb{R}$ and any function $u \in H_{\text {loc }}^{s}\left(\mathbb{R}^{d}\right)$, one can define

$$
\|u\|_{H_{u l}^{s}\left(\mathbb{R}^{d}\right)}=\sup _{x \in \mathbb{R}^{d}}\|\phi(\cdot-x) u(\cdot)\|_{H^{s}\left(\mathbb{R}^{d}\right)} .
$$


In Section 7 and later sections, we need to use Lorentz spaces. We recall the definitions here. For a measurable function $f$, the nonincreasing rearrangement $f^{*}$ is defined by

$$
f^{*}(t)=\inf \{s: \operatorname{Leb}(x:|f(x)|>s) \leq t\} .
$$

For $1 \leq p, q<\infty$, the Lorentz space $L^{p, q}$ is the set of functions $f$ which satisfy

$$
\|f\|_{L^{p, q}}:=\left(\int_{0}^{\infty}\left(t^{\frac{1}{p}} f^{*}(t)\right)^{q} \frac{d t}{t}\right)^{\frac{1}{q}}<\infty .
$$

For $q=\infty, L^{p, \infty}$ is the set of functions such that

$$
\|f\|_{L^{p, \infty}}=\sup _{t>0} t^{\frac{1}{p}} f^{*}(t)<\infty .
$$

For $p=\infty$, we set $L^{\infty, q}=L^{\infty}$ for all $1 \leq q \leq \infty$. Note that $L^{p, p}=L^{p}$. For $1<p<\infty$, the space $L^{p, q}$ coincides with the real interpolation from Lebesgue spaces.

We will need to use the Littlewood-Paley frequency projection operators. Let $\varphi(\xi)$ be a smooth bump function supported in the ball $|\xi| \leq 2$ and equal to one on the ball $|\xi| \leq 1$. For any real number $N>0$ and $f \in \mathcal{S}^{\prime}\left(\mathbb{R}^{d}\right)$, define the frequency localized (LP) projection operators:

$$
\begin{aligned}
\widehat{P_{\leq N} f}(\xi) & :=\varphi(\xi / N) \hat{f}(\xi), \\
\widehat{P_{>N} f}(\xi) & :=[1-\varphi(\xi / N)] \hat{f}(\xi), \\
\widehat{P_{N} f}(\xi) & :=[\varphi(\xi / N)-\varphi(2 \xi / N)] \hat{f}(\xi) .
\end{aligned}
$$

Similarly we can define $P_{<N}, P_{\geq N}$, and $P_{M<\cdot \leq N}:=P_{\leq N}-P_{\leq M}$, whenever $N>$ $M>0$ are real numbers. We will usually use these operators when $M$ and $N$ are dyadic numbers. The summation over $N$ or $M$ are understood to be over dyadic numbers. Occasionally for convenience of notation we allow $M$ and $N$ not to be a power of 2 .

We recall the following Bernstein estimates: for any $1 \leq p \leq q \leq \infty, s \in \mathbb{R}$,

$$
\begin{aligned}
& \left\||\nabla|{ }^{s} P_{N} f\right\|_{L_{x}^{p}\left(\mathbb{R}^{d}\right)} \sim N^{s}\left\|P_{N} f\right\|_{L_{x}^{p}\left(\mathbb{R}^{d}\right)}, \\
& \left\|P_{\leq N} f\right\|_{L_{x}^{q}\left(\mathbb{R}^{d}\right)} \lesssim_{d} N^{d\left(\frac{1}{p}-\frac{1}{q}\right)}\left\|P_{\leq N} f\right\|_{L_{x}^{p}\left(\mathbb{R}^{d}\right)}, \\
& \left\|P_{N} f\right\|_{L_{x}^{q}\left(\mathbb{R}^{d}\right)} \lesssim_{d} N^{d\left(\frac{1}{p}-\frac{1}{q}\right)}\left\|P_{N} f\right\|_{L_{x}^{p}\left(\mathbb{R}^{d}\right)} .
\end{aligned}
$$

For any $s \in \mathbb{R}, 1 \leq p, q \leq \infty$, we define the homogeneous Besov seminorm as

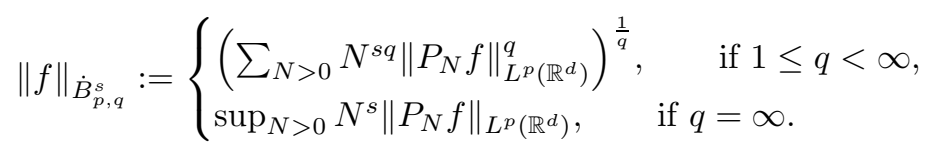

The inhomogeneous Besov norm $\|f\|_{B_{p, q}^{s}}$ of $f \in \mathcal{S}^{\prime}\left(\mathbb{R}^{d}\right)$ is

$$
\|f\|_{B_{p, q}^{s}}=\|f\|_{p}+\|f\|_{\dot{B}_{p, q}^{s}} .
$$

For any $s \in \mathbb{R}, 1<p<\infty, 1 \leq q \leq \infty$, the homogeneous Triebel-Lizorkin seminorm is defined by

$$
\|f\|_{\dot{F}_{p, q}^{s}}:=\left\{\begin{array}{l}
\left\|\left(\sum_{N>0} N^{s q}\left|P_{N} f\right|^{q}\right)^{\frac{1}{q}}\right\|_{L^{p}}, \quad \text { if } 1 \leq q<\infty, \\
\sup _{N>0} N^{s}\left|P_{N} f\right| \|_{L^{p}}, \quad \text { if } q=\infty .
\end{array}\right.
$$


The inhomogeneous Triebel-Lizorkin norm is

$$
\|f\|_{F_{p, q}^{s}}=\|f\|_{p}+\|f\|_{\dot{F}_{p, q}^{s}} .
$$

\section{LOCAL CONSTRUCTION FOR 2D CASE}

We begin by describing the choice of initial data for the local construction.

Let $\varphi_{0} \in C_{c}^{\infty}\left(\mathbb{R}^{2}\right)$ be a radial bump function such that $\operatorname{supp}\left(\varphi_{0}\right) \subset B(0,1)$ and $0 \leq \varphi_{0} \leq 1$. Define

$$
\eta_{0}\left(x_{1}, x_{2}\right)=\sum_{a_{1}, a_{2}= \pm 1} a_{1} a_{2} \cdot \varphi_{0}\left(\frac{\left(x_{1}-a_{1}, x_{2}-a_{2}\right)}{2^{-10}}\right) .
$$

Clearly by definition $\eta_{0}$ is odd in $x_{1}, x_{2}$, i.e.

$$
\eta_{0}\left(x_{1}, x_{2}\right)=-\eta_{0}\left(-x_{1}, x_{2}\right)=-\eta_{0}\left(x_{1},-x_{2}\right), \quad \forall x=\left(x_{1}, x_{2}\right) \in \mathbb{R}^{2} .
$$

Define for each integer $k \geq 1$,

$$
\eta_{k}(x)=\eta_{0}\left(2^{k} x\right)
$$

Obviously,

$$
\operatorname{supp}\left(\eta_{k}\right) \subset \bigcup_{a_{1}, a_{2}= \pm 1} B\left(\left(2^{-k} a_{1}, 2^{-k} a_{2}\right), 2^{-(k+10)}\right),
$$

so that $\eta_{k}$ and $\eta_{l}$ have disjoint supports for $k \neq l$, and

$$
\left\|\partial^{(\alpha)} \eta_{k}\right\|_{\infty} \lesssim_{\alpha} 2^{k|\alpha|} .
$$

Take any $A \gg 1$ and define the following one parameter family of functions:

$$
h_{A}(x)=\frac{\sqrt{\log A}}{A} \sum_{A \leq k \leq 2 A} \eta_{k}(x) .
$$

It is easy to check

$$
\left\|h_{A}\right\|_{1}+\left\|h_{A}\right\|_{\infty} \lesssim \frac{\sqrt{\log A}}{A}
$$

and

$$
\left\|h_{A}\right\|_{H^{1}} \lesssim \frac{\sqrt{\log A}}{\sqrt{A}} .
$$

Note that in computing the $H^{1}$-norm above, we have a saving of $A^{\frac{1}{2}}$ due to the fact that each composing piece $\eta_{k}$ has $O(1) H^{1}$-norm and they have disjoint supports.

We begin with a simple interpolation lemma.

Lemma 3.1. Let $\mathcal{R}=\mathcal{R}_{i j}$ be a Riesz transform on $\mathbb{R}^{2}$, then

$$
\|\mathcal{R} f\|_{\infty} \lesssim\|f\|_{2}^{\frac{1}{2}}\|\nabla f\|_{\infty}^{\frac{1}{2}}
$$

Proof. By using the Littlewood-Paley decomposition, splitting into dyadic frequencies and the Bernstein inequality, we have

$$
\begin{aligned}
\|\mathcal{R} f\|_{\infty} & \lesssim \sum_{N}\left\|P_{N} f\right\|_{\infty} \\
& \lesssim \sum_{N<N_{0}} N\left\|P_{N} f\right\|_{2}+\sum_{N>N_{0}} N^{-1}\left\|P_{N} \nabla f\right\|_{\infty} \\
& \lesssim N_{0}\|f\|_{2}+N_{0}^{-1}\|\nabla f\|_{\infty} .
\end{aligned}
$$


Choosing $N_{0} \in 2^{\mathbb{Z}}$ such that $N_{0} \sim\left(\frac{\|\nabla f\|_{\infty}}{\|f\|_{2}}\right)^{\frac{1}{2}}$ then yields (3.5).

The following lemma gives the estimates of Riesz transforms of compositions with Lipschitz maps on $\mathbb{R}^{2}$ for the functions $h_{A}$ defined earlier.

Lemma 3.2. Let $\phi: \mathbb{R}^{2} \rightarrow \mathbb{R}^{2}$ be a bi-Lipschitz function satisfying the following conditions:

(i) $\phi(0)=0$.

(ii) $\phi=\left(\phi_{1}, \phi_{2}\right)$ commutes with the reflection map $\sigma_{2}\left(x_{1}, x_{2}\right)=\left(x_{1},-x_{2}\right)$, i.e.

$$
\begin{aligned}
& \phi_{1}\left(x_{1},-x_{2}\right)=\phi_{1}\left(x_{1}, x_{2}\right), \\
& \phi_{2}\left(x_{1},-x_{2}\right)=-\phi_{2}\left(x_{1}, x_{2}\right), \quad \forall x=\left(x_{1}, x_{2}\right) \in \mathbb{R}^{2} .
\end{aligned}
$$

(iii) For some integer $n_{0} \geq 1$,

$$
\|D \phi\|_{\infty} \leq 2^{n_{0}} \text { and }\left\|D\left(\phi^{-1}\right)\right\|_{\infty} \leq 2^{n_{0}} .
$$

Here $\phi^{-1}$ denotes the inverse map of $\phi$. Note that equivalently we can write

$$
\left\|(D \phi)^{-1}\right\|_{\infty} \leq 2^{n_{0}}
$$

where $(D \phi)^{-1}$ is the matrix inverse of $D \phi$.

Then with $w=h_{A}$ defined in (3.4), we have

$$
\begin{aligned}
& \left\|\mathcal{R}_{11}(\omega \circ \phi)\right\|_{\infty} \leq C \cdot 3^{n_{0}} \cdot \frac{\sqrt{\log A}}{A}, \\
& \left\|\mathcal{R}_{22}(\omega \circ \phi)\right\|_{\infty} \leq C \cdot 3^{n_{0}} \cdot \frac{\sqrt{\log A}}{A} .
\end{aligned}
$$

Here $C>0$ is an absolute constant. $\mathcal{R}_{11}=\Delta^{-1} \partial_{11}$ and $\mathcal{R}_{22}=\Delta^{-1} \partial_{22}$ are the Riesz transforms.

Remark 3.3. The same result holds if $\phi$ commutes with the map $\sigma_{1}\left(x_{1}, x_{2}\right)=$ $\left(-x_{1}, x_{2}\right)$. Note also that in the proof below, we only used the oddness in $x_{2}$ of $h_{A}$ defined in (3.4).

Proof of Lemma 3.2. First, note that by assumption (ii) on the map $\phi$, the function $\eta_{k} \circ \phi$ is still odd in $x_{2}$. Since $\mathcal{R}_{11}$ is an even operator, it follows that $\mathcal{R}_{11}\left(\eta_{k} \circ \phi\right)(0)=$ 0 . (More precisely one just recalls that $\mathcal{R}_{11}$ is obtained by convolution with the even kernel $K(x)=p . v \cdot\left(\frac{1}{2 \pi} \cdot \frac{x_{2}^{2}-x_{1}^{2}}{\left(x_{1}^{2}+x_{2}^{2}\right)^{2}}\right)+\frac{1}{2} \delta(x)$, and $\mathcal{R}_{11}\left(\eta_{k} \circ \phi\right)(0)=\left\langle\eta_{k} \circ \phi, K\right\rangle=0$.)

Now let $x \in \mathbb{R}^{2} \backslash\{0\},|x| \sim 2^{-l}$. We evaluate $\mathcal{R}_{11}\left(\eta_{k} \circ \phi\right)(x)$ by considering 3 cases.

Case 1. $2^{k} \ll 2^{l-n_{0}}$. (see (3.6) for the definition of $n_{0}$.)

By definition

$$
\left|\mathcal{R}_{11}\left(\eta_{k} \circ \phi\right)(x)\right|=\left|\int_{\mathbb{R}^{2}}\left(\eta_{k} \circ \phi\right)(x-y) K(y) d y\right| .
$$

The integrand in (3.9) vanishes unless $|\phi(x-y)| \sim 2^{-k}$ (see (3.2)). By (3.6) and $\phi(0)=0$, we have

$$
2^{-k+n_{0}} \gtrsim|x-y| \gtrsim 2^{-k-n_{0}} \gg 2^{-l} .
$$

Therefore $2^{-k-n_{0}} \lesssim|y| \lesssim 2^{-k+n_{0}}$. Since $\left(\eta_{k} \circ \phi\right)\left(y_{1}, y_{2}\right)$ is odd in the $y_{2}$ variable, obviously

$$
\int_{2^{-k-n_{0}} \lesssim|y| \lesssim 2^{-k+n_{0}}}\left(\eta_{k} \circ \phi\right)(-y) K(y) d y=0 .
$$


We then insert the above into (3.9) and compute

$$
\begin{aligned}
\left|\mathcal{R}_{11}\left(\eta_{k} \circ \phi\right)(x)\right| & \leq \int_{2^{-k-n_{0}} \lesssim|y| \lesssim 2^{-k+n_{0}}}\left|\left(\eta_{k} \circ \phi\right)(x-y)-\left(\eta_{k} \circ \phi\right)(-y) \| K(y)\right| d y \\
& \leq|x| \cdot\left\|\nabla\left(\eta_{k} \circ \phi\right)\right\|_{\infty} \cdot \int_{2^{-k-n_{0}} \lesssim|y| \lesssim 2^{-k+n_{0}}}|K(y)| d y \\
& \lesssim 2^{-l} \cdot 2^{n_{0}} \cdot 2^{k} \cdot n_{0} .
\end{aligned}
$$

Case 2. $2^{k} \gg 2^{l+n_{0}}$.

Again the integrand in (3.9) vanishes unless $|\phi(x-y)| \sim 2^{-k}$ which yields $2^{-k+n_{0}} \gtrsim|x-y| \gtrsim 2^{-k-n_{0}}$. Since $2^{-l} \gg 2^{-k+n_{0}}$ and $|x| \sim 2^{-l}$, we get $|y| \sim 2^{-l}$. Therefore

$$
\begin{aligned}
\left|\mathcal{R}_{11}\left(\eta_{k} \circ \phi\right)(x)\right| & \leq\|K\|_{L^{\infty}\left(|y| \sim 2^{-l}\right)} \cdot\left\|\eta_{k} \circ \phi\right\|_{1} \\
& \lesssim 4^{l} \cdot 4^{-k} \cdot 4^{n_{0}}=4^{-k+l+n_{0}} .
\end{aligned}
$$

Case 3. $2^{l-n_{0}} \lesssim 2^{k} \lesssim 2^{l+n_{0}}$.

In this case we use Lemma 3.1. Then by (3.5) and (3.3),

$$
\begin{aligned}
\left\|\mathcal{R}_{11}\left(\eta_{k} \circ \phi\right)\right\|_{\infty} & \lesssim\left\|\eta_{k} \circ \phi\right\|_{2}^{\frac{1}{2}} \cdot\left\|\nabla\left(\eta_{k} \circ \phi\right)\right\|_{\infty}^{\frac{1}{2}} \\
& \lesssim 2^{\frac{1}{2} n_{0}} \cdot\left\|\eta_{k}\right\|_{2}^{\frac{1}{2}} \cdot\left\|\nabla \eta_{k}\right\|_{\infty}^{\frac{1}{2}} \cdot 2^{\frac{1}{2} n_{0}} \\
& \lesssim 2^{n_{0}} \cdot 2^{-\frac{k}{2}} \cdot 2^{\frac{k}{2}} \lesssim 2^{n_{0}}
\end{aligned}
$$

Collecting all the estimates, we then obtain

$$
\begin{aligned}
\sum_{k}\left|\mathcal{R}_{11}\left(\eta_{k} \circ \phi\right)(x)\right| & \lesssim 2^{n_{0}} \cdot n_{0}+n_{0} \\
& \lesssim 3^{n_{0}} .
\end{aligned}
$$

The bound (3.7) follows from this and the normalizing factor in (3.4). Similarly one can prove (3.8) or just use the identity $\mathcal{R}_{11}+\mathcal{R}_{22}=I d$.

Since we will be dealing with symplectic maps later on, we now state a symplectic variant of Lemma 3.2 .

Lemma 3.4. Let $\phi: \mathbb{R}^{2} \rightarrow \mathbb{R}^{2}$ be a smooth symplectic (i.e. $\operatorname{det}(D \phi) \equiv 1$ ) function satisfying the following conditions:

(i) $\phi(0)=0$.

(ii) $\phi$ commutes with $\sigma_{2}\left(x_{1}, x_{2}\right)=\left(x_{1},-x_{2}\right)$.

(iii) For some integer $n_{0} \geq 1$,

$$
\|D \phi\|_{\infty} \leq 2^{n_{0}} .
$$

Then with $w=h_{j}$ defined in (3.4), we have

$$
\begin{aligned}
& \left\|\mathcal{R}_{11}(\omega \circ \phi)\right\|_{\infty} \leq C \cdot 2^{n_{0}} \cdot \frac{\sqrt{\log A}}{A}, \\
& \left\|\mathcal{R}_{22}(\omega \circ \phi)\right\|_{\infty} \leq C \cdot 2^{n_{0}} \cdot \frac{\sqrt{\log A}}{A} .
\end{aligned}
$$

Here $C>0$ is an absolute constant. 
Proof of Lemma 3.4. This is essentially a repetition of the proof of Lemma 3.2. Note that by sympleticity, (3.11) implies that $\left\|(D \phi)^{-1}\right\|_{\infty} \leq 2^{n_{0}}$. Also there is a slight improvement of constant in (3.12) (3.13). This is because when bounding (3.10) we no longer need to bound the Jacobian since the map is volume-preserving.

We are now ready to describe the details of the local construction: namely the existence of large deformation for well-chosen initial data.

To be more specific, we consider the Euler equation

$$
\left\{\begin{array}{l}
\partial_{t} \omega+\left(\Delta^{-1} \nabla^{\perp} \omega \cdot \nabla\right) \omega=0, \quad t>0, \\
\left.\omega\right|_{t=0}=h_{A}
\end{array}\right.
$$

where $h_{A}$ is defined in (3.4). Easy to check that $\omega$ is odd in both $x_{1}$ and $x_{2}$. We suppress the dependence of the solution $\omega$ on the parameter $A$ for simplicity of notation.

The equation for the (forward) characteristic lines takes the form

$$
\left\{\begin{array}{l}
\partial_{t} \phi(t, x)=\left(\Delta^{-1} \nabla^{\perp} \omega\right)(t, \phi(t, x)), \\
\phi(0, x)=x \in \mathbb{R}^{2} .
\end{array}\right.
$$

It is easy to check that $\phi=\phi(t, x)$ is a symplectic map and $\phi(t, 0) \equiv 0$. Due to the special choice of the initial data $h_{A}$, the flow associated with (3.14) and (3.15) is hyperbolic near the origin with a large deformation gradient. The following proposition quantifies this fact.

Proposition 3.5. With the notations in (3.14)-(3.15), we have for A sufficiently large,

$$
\max _{0 \leq t \leq t_{A}}\|(D \phi)(t, \cdot)\|_{\infty}>M_{A},
$$

where $M_{A}=\log \log A$ and $t_{A}=1 / \log \log A$.

Proof of Proposition 3.5. We shall argue by contradiction. Assume that

$$
\max _{0 \leq t \leq t_{A}}\|(D \phi)(t, \cdot)\|_{\infty} \leq M_{A} .
$$

By Lemma 3.4 we have

$$
\begin{aligned}
& \max _{0 \leq t \leq t_{A}}\left\|\mathcal{R}_{11} \omega\right\|_{\infty} \lesssim M_{A} \frac{\sqrt{\log A}}{A}, \\
& \max _{0 \leq t \leq t_{A}}\left\|\mathcal{R}_{22} \omega\right\|_{\infty} \lesssim M_{A} \frac{\sqrt{\log A}}{A} .
\end{aligned}
$$

Denote $D(t)=D(t, \cdot)=(D \phi)(t, \cdot)$. By (3.15) and (3.18), we have

$$
\begin{aligned}
\partial_{t} D(t) & =\left(\begin{array}{cc}
-\mathcal{R}_{12} \omega & -\mathcal{R}_{22} \omega \\
\mathcal{R}_{11} \omega & \mathcal{R}_{12} \omega
\end{array}\right) D(t) \\
& =:\left(\begin{array}{cc}
-\lambda(t) & 0 \\
0 & \lambda(t)
\end{array}\right) D(t)+P(t) D(t),
\end{aligned}
$$

where $\lambda(t, x)=\left(\mathcal{R}_{12} \omega\right)(t, \phi(t, x))$, and

$$
\max _{0 \leq t \leq t_{A}}\|P(t)\|_{\infty} \lesssim M_{A} \frac{\sqrt{\log A}}{A} .
$$


Integrating (3.19) in time and noting that $D(0)=I d$, we get

$$
D(t)=\left(\begin{array}{cc}
e^{-\int_{0}^{t} \lambda} & 0 \\
0 & e^{\int_{0}^{t} \lambda}
\end{array}\right)+\int_{0}^{t}\left(\begin{array}{cc}
e^{-\int_{\tau}^{t} \lambda} & 0 \\
0 & e^{\int_{\tau}^{t} \lambda}
\end{array}\right) P(\tau) D(\tau) d \tau .
$$

By (3.17), (3.20) and (3.21), we have for all $0 \leq t \leq t_{A}$,

$$
e^{\left|\int_{0}^{t} \lambda\right|} \leq M_{A}+C_{2} \cdot M_{A}^{2} \cdot \frac{\sqrt{\log A}}{A} \cdot \max _{0 \leq \tau \leq t}\left(e^{2\left|\int_{0}^{\tau} \lambda\right|}\right),
$$

where $C_{2}>0$ is some absolute constant.

By taking $A$ sufficiently large and a standard continuity argument, we get

$$
e^{\left|\int_{0}^{t} \lambda\right|} \leq 2 M_{A}, \quad \forall 0 \leq t \leq t_{A} .
$$

Now denote

$$
D=\left(\begin{array}{cc}
e^{-\alpha} & 0 \\
0 & e^{\alpha}
\end{array}\right)+\beta,
$$

where $\alpha(t, x):=\int_{0}^{t} \lambda(\tau, x) d \tau$ and

$$
|\beta| \leq C_{2} \cdot M_{A}^{2} \cdot \frac{\sqrt{\log A}}{A} \cdot 4 M_{A}^{2} .
$$

From (3.23) we can get more information on the transport map $\phi=\phi(t, x)$. Indeed for fixed $t$, using the fact that $\phi(t, 0) \equiv 0$, we have

$$
\begin{aligned}
\phi(t, x) & =\phi(t, x)-\phi(t, 0) \\
& =\int_{0}^{1} \frac{d}{d s}(\phi(t, s x)) d s \\
& =\left(\int_{0}^{1}(D \phi)(t, s x) d s\right) x \\
& =\left(\left(\int_{0}^{1} e^{-\alpha(t, s x)} d s\right) x_{1},\left(\int_{0}^{1} e^{\alpha(t, s x)} d s\right) x_{2}\right)+\tilde{\beta},
\end{aligned}
$$

where

$$
|\tilde{\beta}| \lesssim M_{A}^{4} \cdot \frac{\sqrt{\log A}}{A} \cdot|x| .
$$

Note that by (3.22), for any $0 \leq t \leq t_{A}$,

$$
\begin{gathered}
\frac{1}{2 M_{A}} \leq \int_{0}^{1} e^{\alpha(t, s x)} d s \leq 2 M_{A}, \\
\frac{1}{2 M_{A}} \leq \int_{0}^{1} e^{-\alpha(t, s x)} d s \leq 2 M_{A} .
\end{gathered}
$$

Since

$$
M_{A}^{4} \cdot \frac{\sqrt{\log A}}{A} \ll \frac{1}{M_{A}},
$$

we have if $x_{1}>0, x_{2}>0$, and

$$
\frac{1}{2}<\frac{x_{1}}{x_{2}}<2
$$


then for $\phi(t, x)=\left(\phi_{1}(t, x), \phi_{2}(t, x)\right), 0 \leq t \leq t_{A}$,

$$
\frac{1}{10 M_{A}^{2}}<\frac{\phi_{1}(t, x)}{\phi_{2}(t, x)}<10 M_{A}^{2}
$$

By (3.17), we also have

$$
|\phi(t, x)| \leq M_{A}|x| .
$$

These bounds will be needed later.

Now we analyze $\lambda(t, \cdot)$ at $x=0$ to get a contradiction. We have (recall $\omega(0, x)=$ $\left.h_{A}(x)\right)$

$$
\begin{aligned}
\lambda(t, 0) & =\left(\mathcal{R}_{12} \omega\right)(t, \phi(t, 0))=\left(\mathcal{R}_{12} \omega\right)(t, 0) \\
& =-\frac{1}{\pi} \int_{\mathbb{R}^{2}} \omega(t, x) \frac{x_{1} x_{2}}{\left(x_{1}^{2}+x_{2}^{2}\right)^{2}} d x \\
& =-\frac{1}{\pi} \int_{\mathbb{R}^{2}} h_{A}(x) \cdot \frac{\phi_{1}(t, x) \phi_{2}(t, x)}{\left(\phi_{1}(t, x)^{2}+\phi_{2}(t, x)^{2}\right)^{2}} d x .
\end{aligned}
$$

In the last step above we have made a change of variable $x \rightarrow \phi(t, x)$ and used the fact $\omega(t, \phi(t, x))=\omega(0, x)=h_{A}(x)$.

To continue, let us observe that the maps $\phi_{1}$ and $\phi_{2}$ are sign-preserving, i.e. if $x_{1} \geq 0$ (resp. $\left.x_{2} \geq 0\right)$ then $\phi_{1} \geq 0\left(\operatorname{resp} \phi_{2} \geq 0\right)$. To check this, one can use (3.15) and the fact that $\omega$ is odd in $x_{1}$ and $x_{2}$ to get

$$
\begin{aligned}
\partial_{t} \phi_{1} & =\left(-\Delta^{-1} \partial_{2} \omega\right)\left(t, \phi_{1}, \phi_{2}\right)-\left(-\Delta^{-1} \partial_{2} \omega\right)\left(t, 0, \phi_{2}\right) \\
& =F\left(t, \phi_{1}, \phi_{2}\right) \phi_{1},
\end{aligned}
$$

which (by integrating in time) yields that $\operatorname{sign}\left(\phi_{1}(t)\right)=\operatorname{sign}\left(\phi_{1}(0)\right)=\operatorname{sign}\left(x_{1}\right)$.

By using the sign property mentioned above and the parity of our solution, we conclude that the RHS integral of (3.26) is always non-negative and can be restricted to the first quadrant. Hence by (3.26), (3.24) and (3.25), we have for all $0 \leq t \leq t_{A}$,

$$
\begin{aligned}
-\frac{\pi}{4} \lambda(t, 0) & =\int_{x_{1}>0, x_{2}>0} h_{A}(x) \cdot \frac{\phi_{1}(t, x) \phi_{2}(t, x)}{\left(\phi_{1}^{2}(t, x)+\phi_{2}^{2}(t, x)\right)^{2}} d x \\
& =\int_{x_{1}>0, x_{2}>0} h_{A}(x) \cdot \frac{1}{\frac{\phi_{1}(t, x)}{\phi_{2}(t, x)}+\frac{\phi_{2}(t, x)}{\phi_{1}(t, x)}} \cdot \frac{1}{\phi_{1}^{2}(t, x)+\phi_{2}^{2}(t, x)} d x \\
& \geq \int_{x_{1}>0, x_{2}>0} h_{A}(x) \cdot \frac{1}{20 M_{A}^{2}} \cdot \frac{1}{M_{A}^{2}} \cdot \frac{1}{|x|^{2}} d x \\
& \gtrsim \frac{1}{M_{A}^{4}} \cdot \frac{\sqrt{\log A}}{A} \cdot \sum_{A \leq k \leq 2 A} \int_{x_{1}>0, x_{2}>0} \frac{\eta_{k}(x)}{|x|^{2}} d x \\
& \gtrsim M_{A}^{-4} \cdot \sqrt{\log A} .
\end{aligned}
$$

Therefore

$$
\int_{0}^{t_{A}} \lambda(t, 0) d t \gtrsim t_{A} \cdot M_{A}^{-4} \cdot \sqrt{\log A}
$$

which obviously contradicts (3.22).

The special initial data $h_{A}$ in Proposition 3.5 can be generalized to a slightly larger class of functions. Also the proof of Proposition 3.5 can be simplified if we take full advantage of the odd symmetry of the data. The main observation is that 
by parity $x=0$ is invariant under the flow and $(D u)(t, 0)$ is diagonal for all $t>0$. We now state a more general result taking into account all these considerations. The argument below bypasses Lemma 3.2 and is more streamlined and quantitative. In particular the contradiction argument is replaced by a more effective integral (in time) inequality.

Consider

$$
\left\{\begin{array}{l}
\partial_{t} \omega+\left(\Delta^{-1} \nabla^{\perp} \omega \cdot \nabla\right) \omega=0, \quad t>0 \\
\left.\omega\right|_{t=0}=g
\end{array}\right.
$$

Assume $g \in C_{c}^{\infty}\left(\mathbb{R}^{2}\right)$ satisfies

(i) $g$ is odd in $x_{1}$ and $x_{2}$, and

$$
g\left(x_{1}, x_{2}\right) \geq 0, \quad \text { if } x_{1} \geq 0 \text { and } x_{2} \geq 0 .
$$

(ii)

$$
\int_{\mathbb{R}^{2}} g(x) \frac{x_{1} x_{2}}{|x|^{4}} d x=B>0 .
$$

Denoting by $\phi=\phi(t, x)$ the (forward) characteristic lines, we have

\section{Proposition 3.6.}

$$
\int_{0}^{t} \frac{1}{\|D \phi(s)\|_{\infty}^{4}} d s \leq \frac{\pi}{4 B} \log \left(1+\frac{4 B}{\pi} t\right), \quad \forall t \geq 0 .
$$

In particular,

$$
\max _{0 \leq s \leq t}\|D \phi(s)\|_{\infty} \geq\left(\frac{4 B}{\pi} \cdot \frac{t}{\log \left(1+\frac{4 B}{\pi} t\right)}\right)^{\frac{1}{4}}, \quad \forall t>0 .
$$

Proof of Proposition 3.6. By parity, we have $\phi(t, 0) \equiv 0$ and

$$
(D u)(t, 0)=\left(\begin{array}{cc}
-\lambda(t) & 0 \\
0 & \lambda(t)
\end{array}\right),
$$

where $\lambda(t)=\left(\mathcal{R}_{12} \omega\right)(t, 0)$. The off-diagonal terms of $D u$ vanishes at $x=0$ since $\mathcal{R}_{11} \omega$ and $\mathcal{R}_{22} \omega$ are both odd functions of $x_{1}, x_{2}$. Integrating in time gives

$$
(D \phi)(t, 0)=\left(\begin{array}{cc}
e^{-\int_{0}^{t} \lambda(\tau) d \tau} & 0 \\
0 & e^{\int_{0}^{t} \lambda(\tau) d \tau}
\end{array}\right)
$$

Write $\phi=\left(\phi_{1}, \phi_{2}\right)$. By parity it is easy to check $\phi_{1}\left(t, 0, x_{2}\right) \equiv 0, \phi_{2}\left(t, x_{1}, 0\right) \equiv 0$ for any $x_{1}, x_{2} \in \mathbb{R}$. By this and sign preservation it follows that for any $x_{1} \geq 0$, $x_{2} \geq 0$,

$$
\begin{aligned}
& \frac{1}{\|D \phi(t)\|_{\infty}} \phi_{1}\left(t, x_{1}, x_{2}\right) \leq x_{1} \leq \phi_{1}\left(t, x_{1}, x_{2}\right) \cdot\|D \phi(t)\|_{\infty}, \\
& \frac{1}{\|D \phi(t)\|_{\infty}} \phi_{2}\left(t, x_{1}, x_{2}\right) \leq x_{2} \leq \phi_{2}\left(t, x_{1}, x_{2}\right) \cdot\|D \phi(t)\|_{\infty} .
\end{aligned}
$$


Therefore for any $x_{1}>0, x_{2}>0$,

$$
\begin{aligned}
\frac{\phi_{1} \phi_{2}}{\left(\phi_{1}^{2}+\phi_{2}^{2}\right)^{2}} & =\frac{1}{\frac{\phi_{1}}{\phi_{2}}+\frac{\phi_{2}}{\phi_{1}}} \cdot \frac{1}{\phi_{1}^{2}+\phi_{2}^{2}} \\
& \geq \frac{1}{\|D \phi\|_{\infty}^{4}} \cdot \frac{1}{\frac{x_{1}}{x_{2}}+\frac{x_{2}}{x_{1}}} \cdot \frac{1}{|x|^{2}} \\
& =\frac{1}{\|D \phi\|_{\infty}^{4}} \cdot \frac{x_{1} x_{2}}{|x|^{4}} .
\end{aligned}
$$

We compute $\lambda(t)$ as

$$
\begin{aligned}
-\pi \lambda(t) & =\int_{\mathbb{R}^{2}} g(x) \frac{\phi_{1}(t, x) \phi_{2}(t, x)}{|\phi(t, x)|^{4}} d x \\
& \geq 4 \int_{x_{1}>0, x_{2}>0} g(x) \frac{\phi_{1}(t, x) \phi_{2}(t, x)}{|\phi(t, x)|^{4}} d x \\
& \geq \frac{4}{\|D \phi(t)\|_{\infty}^{4}} \int_{x_{1}>0, x_{2}>0} g(x) \frac{x_{1} x_{2}}{|x|^{4}} d x \\
& =\frac{B}{\|D \phi(t)\|_{\infty}^{4}} .
\end{aligned}
$$

Since

$$
\|D \phi(t, \cdot)\|_{\infty} \geq\|(D \phi)(t, 0)\|_{\infty} \geq \exp \left(-\int_{0}^{t} \lambda(s) d s\right),
$$

we get

$$
\|D \phi(t)\|_{\infty} \geq \exp \left(\frac{B}{\pi} \int_{0}^{t} \frac{1}{\|D \phi(s)\|_{\infty}^{4}} d s\right) .
$$

Equivalently,

$$
\frac{d}{d t}\left(\exp \left(\frac{4 B}{\pi} \int_{0}^{t} \frac{1}{\|D \phi(s)\|_{\infty}^{4}} d s\right)\right) \leq \frac{4 B}{\pi}, \quad \forall t \geq 0 .
$$

Integrating in time, we get

$$
\int_{0}^{t} \frac{1}{\|D \phi(s)\|_{\infty}^{4}} d s \leq \frac{\pi}{4 B} \log \left(1+\frac{4 B}{\pi} t\right), \quad \forall t \geq 0 .
$$

\section{4. $\dot{H}^{1}$ NORM INFLATION BY LARGE LAGRANGIAN DEFORMATION}

We begin with a simple ODE perturbation lemma.

Lemma 4.1. Suppose $u=u(t, x): \mathbb{R} \times \mathbb{R}^{2} \rightarrow \mathbb{R}, v=v(t, x): \mathbb{R} \times \mathbb{R}^{2} \rightarrow \mathbb{R}$ are given smooth vector fields. Let $\phi_{1}, \phi_{2}$ solve respectively

$$
\left\{\begin{array}{l}
\partial_{t} \phi_{1}(t, x)=u\left(t, \phi_{1}(t, x)\right), \\
\phi_{1}(0, x)=x \in \mathbb{R}^{2}
\end{array}\right.
$$

and

$$
\left\{\begin{array}{l}
\partial_{t} \phi_{2}(t, x)=u\left(t, \phi_{2}(t, x)\right)+v\left(t, \phi_{2}(t, x)\right), \\
\phi_{2}(0, x)=x \in \mathbb{R}^{2}
\end{array}\right.
$$


Then for some constant $C=C\left(\max _{0 \leq t \leq 1}\left\|D^{2} u(t)\right\|_{\infty}, \max _{0 \leq t \leq 1}\|D u(t)\|_{\infty}\right)>$ 0 , we have

$$
\begin{array}{r}
\max _{0 \leq t \leq 1}\left(\left\|\phi_{2}(t, \cdot)-\phi_{1}(t, \cdot)\right\|_{\infty}+\left\|\left(D \phi_{2}\right)(t)-\left(D \phi_{1}\right)(t)\right\|_{\infty}\right) \leq \\
C \cdot \max _{0 \leq t \leq 1}\left(\|v(t)\|_{\infty}+\|D v(t)\|_{\infty}\right) .
\end{array}
$$

Proof of Lemma 4.1. This is quite standard. We sketch the details for the sake of completeness.

Set $\eta(t, x)=\phi_{2}(t, x)-\phi_{1}(t, x)$. Then

$$
\begin{aligned}
\partial_{t} \eta & =u\left(t, \phi_{2}\right)-u\left(t, \phi_{1}\right)+v\left(t, \phi_{2}\right) \\
& =\int_{0}^{1}(D u)\left(t, \phi_{1}+\left(\phi_{2}-\phi_{1}\right) \theta\right) d \theta \eta+v\left(t, \phi_{2}\right) .
\end{aligned}
$$

A Gronwall in time argument then yields

$$
\max _{0 \leq t \leq 1}\|\eta(t)\|_{\infty} \leq C \max _{0 \leq t \leq 1}\|v(t)\|_{\infty}
$$

where the constant $C=C\left(\max _{0 \leq t \leq 1}\|D u(t)\|_{\infty}\right)$.

Now for $\partial_{x} \eta$ note that

$$
\begin{aligned}
\partial_{t}(D \eta) & =(D u)\left(t, \phi_{2}\right) D \phi_{2}-(D u)\left(t, \phi_{1}\right) D \phi_{1}+(D v)\left(t, \phi_{2}\right) D \phi_{2} \\
& =\left((D u)\left(t, \phi_{2}\right)-(D u)\left(t, \phi_{1}\right)\right) D \phi_{2}+(D u)\left(t, \phi_{1}\right) D \eta+(D v) D \phi_{2} \\
& =O\left(\left\|D^{2} u\right\|_{\infty} \cdot\|\eta\|_{\infty} \cdot\left\|D \phi_{2}\right\|_{\infty}\right)+O\left(\|D u\|_{\infty}\right) D \eta+O\left(\|D v\|_{\infty} \cdot\left\|D \phi_{2}\right\|_{\infty}\right) .
\end{aligned}
$$

It is easy to estimate

$$
\max _{0 \leq t \leq 1}\left\|\left(D \phi_{2}\right)(t, \cdot)\right\|_{\infty} \leq \exp \left(\text { const } \cdot\left(\max _{0 \leq t \leq 1}\left(\|D u(t)\|_{\infty}+\|D v(t)\|_{\infty}\right)\right)\right) .
$$

Hence the desired bound follows from Gronwall.

The following key proposition shows that large deformation of the transportation map can produce large $\dot{H}^{1}$ norm, provided we perturb the initial data judiciously.

Proposition 4.2 (Large deformation induces $\dot{H}^{1}$ inflation). Suppose $\omega$ is a smooth solution to the Euler equation

$$
\left\{\begin{array}{l}
\partial_{t} \omega+\Delta^{-1} \nabla^{\perp} \omega \cdot \nabla \omega=0, \quad 0<t \leq 1, \\
\left.\omega\right|_{t=0}=\omega_{0}
\end{array}\right.
$$

satisfying the following conditions:

- $\left\|\omega_{0}\right\|_{L^{1}}+\left\|\omega_{0}\right\|_{L^{\infty}}+\left\|\omega_{0}\right\|_{\dot{H}^{-1}}<\infty$.

- For some $z_{0} \in \mathbb{R}^{2}, R_{0}>0$, we have

$$
\operatorname{supp}(\omega(t, \cdot)) \subset B\left(z_{0}, \frac{1}{2} R_{0}\right), \quad \forall 0 \leq t \leq 1 .
$$

- For some $0<t_{0} \leq 1$ and some $M \gg 1$ ( $M \geq 10^{7}$ will suffice), we have

$$
\left\|(D \phi)\left(t_{0}, \cdot\right)\right\|_{\infty}>M
$$

where $\phi=\phi(t, x)$ is the (forward) characteristics:

$$
\left\{\begin{array}{l}
\partial_{t} \phi(t, x)=\left(\Delta^{-1} \nabla^{\perp} \omega\right)(t, \phi(t, x)), \\
\phi(0, x)=x .
\end{array}\right.
$$


Then we can find a smooth solution $\tilde{\omega}$ also solving the Euler equation

$$
\left\{\begin{array}{l}
\partial_{t} \tilde{\omega}+\Delta^{-1} \nabla^{\perp} \tilde{\omega} \cdot \nabla \tilde{\omega}=0, \quad 0<t \leq 1 \\
\left.\tilde{\omega}\right|_{t=0}=\tilde{\omega}_{0}
\end{array}\right.
$$

such that the following hold

(1) $\tilde{\omega}_{0}$ is a small perturbation of $\omega_{0}$ :

$$
\begin{aligned}
\left\|\tilde{\omega}_{0}\right\|_{L^{1}} & \leq 2\left\|\omega_{0}\right\|_{L^{1}}, \\
\left\|\tilde{\omega}_{0}\right\|_{L^{\infty}} & \leq 2\left\|\omega_{0}\right\|_{L^{\infty}}, \\
\left\|\tilde{\omega}_{0}\right\|_{\dot{H}^{-1}} & \leq 2\left\|\omega_{0}\right\|_{\dot{H}^{-1}}, \\
\left\|\tilde{\omega}_{0}\right\|_{\dot{H}^{1}} & \leq\left\|\omega_{0}\right\|_{\dot{H}^{1}}+M^{-\frac{1}{2}} .
\end{aligned}
$$

(2) For the same $t_{0}$ as in (4.1), we have

$$
\left\|\tilde{\omega}\left(t_{0}, \cdot\right)\right\|_{\dot{H}^{1}}>M^{\frac{1}{3}} .
$$

(3) $\tilde{\omega}$ is also compactly supported:

$$
\operatorname{supp}(\tilde{\omega}(t)) \subset B\left(z_{0}, R_{0}\right), \quad \forall 0 \leq t \leq 1 .
$$

Proof of Proposition 4.2. To simplify the later computation, we begin with a general derivation. Let $W=W(t, x)$ be a smooth solution to the Euler equation

$$
\left\{\begin{array}{l}
\partial_{t} W+\Delta^{-1} \nabla^{\perp} W \cdot \nabla W=0, \\
\left.W\right|_{t=0}=f .
\end{array}\right.
$$

Denote the associated (forward) characteristics as $\Phi=\Phi(t, x)$ which solves

$$
\left\{\begin{array}{l}
\partial_{t} \Phi(t, x)=\left(\Delta^{-1} \nabla^{\perp} W\right)(t, \Phi(t, x)), \\
\Phi(0, x)=x
\end{array}\right.
$$

Let $\tilde{\Phi}(t, x)$ be the inverse map of $\Phi(t, x)$. Then

$$
\tilde{\Phi}(t, \Phi(t, x))=x .
$$

Differentiating the above gives us

$$
(D \tilde{\Phi})(t, \Phi(t, x))(D \Phi)(t, x)=I d
$$

or

$$
(D \tilde{\Phi})(t, \Phi(t, x))=(D \Phi(t, x))^{-1},
$$

where $(D \Phi(t, x))^{-1}$ is the usual matrix inverse.

Since $\Phi(t)$ is a smooth symplectic map with $\Phi(0, x)=x$, we have $\operatorname{det}(D \Phi)=1$. Denote $\Phi(t, x)=\left(\Phi_{1}(t, x), \Phi_{2}(t, x)\right)$ and recall

$$
D \Phi=\left(\begin{array}{ll}
\frac{\partial \Phi_{1}}{\partial x_{1}} & \frac{\partial \Phi_{1}}{\partial x_{2}} \\
\frac{\partial \Phi_{2}}{\partial x_{1}} & \frac{\partial \Phi_{2}}{\partial x_{2}}
\end{array}\right) .
$$

Then

$$
(D \Phi)^{-1}=\left(\begin{array}{ll}
\frac{\partial \Phi_{2}}{\partial x_{2}} & -\frac{\partial \Phi_{1}}{\partial x_{2}} \\
-\frac{\partial \Phi_{2}}{\partial x_{1}} & \frac{\partial \Phi_{1}}{\partial x_{1}}
\end{array}\right) .
$$


Since $W(t, x)=f(\tilde{\Phi}(t, x))$, we get

$$
\begin{aligned}
\int_{\mathbb{R}^{2}}|(D W)(t, x)|^{2} d x & =\int_{\mathbb{R}^{2}}|(D f)(\tilde{\Phi}(t, x))(D \tilde{\Phi})(t, x)|^{2} d x \\
& =\int_{\mathbb{R}^{2}}\left|(D f)(x)(D \Phi(t, x))^{-1}\right|^{2} d x
\end{aligned}
$$

where we have performed a measure-preserving change of variables $x \rightarrow \Phi(t, x)$ and used (4.8).

By (4.9), we can then write (4.10) as

$$
\begin{aligned}
\|W(t, \cdot)\|_{\dot{H}^{1}}^{2}= & \int_{\mathbb{R}^{2}}\left|(\nabla f)(x) \cdot\left(\nabla^{\perp} \Phi_{2}\right)(t, x)\right|^{2} d x \\
& +\int_{\mathbb{R}^{2}}\left|(\nabla f)(x) \cdot\left(\nabla^{\perp} \Phi_{1}\right)(t, x)\right|^{2} d x .
\end{aligned}
$$

We shall need this formula below.

Now discuss two cases.

Case 1: $\left\|\omega\left(t_{0}, \cdot\right)\right\|_{\dot{H}^{1}}>M^{\frac{1}{3}}$. In this case we just set $\tilde{\omega}=\omega$ and no work is needed.

Case 2: $\left\|\omega\left(t_{0}, \cdot\right)\right\|_{\dot{H}^{1}} \leq M^{\frac{1}{3}}$. It is this case which requires a nontrivial analysis. We shall use a perturbation argument.

By (4.1), we can find $x_{*}$ such that

$$
\left\|(D \phi)\left(t_{0}, x_{*}\right)\right\|_{\infty}>M .
$$

Here for a matrix $A=\left(a_{i j}\right),\|A\|_{\infty}:=\max \left|a_{i j}\right|$.

Denote $\phi\left(t_{0}, x\right)=\left(\phi_{1}\left(t_{0}, x\right), \phi_{2}\left(t_{0}, x\right)\right)$. Without loss of generality, we may assume one of the entries of $(D \phi)\left(t_{0}, x_{*}\right)$ is at least $M$, namely

$$
\left|\frac{\partial \phi_{2}}{\partial x_{2}}\left(t_{0}, x_{*}\right)\right|>M
$$

By continuity we can find $\delta>0$ sufficiently small such that $\left\{x:\left|x-x_{*}\right| \leq 2 \delta\right\} \subset$ $B\left(z_{0}, R_{0}\right)$ and

$$
\left|\frac{\partial \phi_{2}}{\partial x_{2}}\left(t_{0}, x\right)\right|>M, \quad \forall\left|x-x_{*}\right| \leq 2 \delta
$$

Now let $\Phi_{0} \in C_{c}^{\infty}\left(\mathbb{R}^{2}\right)$ be a radial bump function such that $0 \leq \Phi_{0}(x) \leq 1$ for all $x \in \mathbb{R}^{2}, \Phi_{0}(x)=1$ for $|x| \leq 1$ and $\Phi_{0}(x)=0$ for $|x| \geq 2$. Obviously

$$
\sqrt{\pi} \leq\left\|\Phi_{0}\right\|_{2} \leq 2 \sqrt{\pi}
$$

Depending on the location of $x_{*}$, we need to shrink $\delta>0$ slightly further if necessary and define an even function $b \in C_{c}^{\infty}\left(\mathbb{R}^{2}\right)$ as follows. If $x_{*}=(0,0)$, we just define

$$
b(x)=\frac{1}{\delta} \Phi_{0}\left(\frac{x}{\delta}\right) .
$$

If $x_{*}=\left(a_{*}, 0\right)$ for some $a_{*} \neq 0$, then we shrink $\delta>0$ such that $\delta \ll\left|a_{*}\right|$ and define

$$
b(x)=\frac{1}{\delta}\left(\Phi_{0}\left(\frac{x-x_{*}}{\delta}\right)+\Phi_{0}\left(\frac{x+x_{*}}{\delta}\right)\right) .
$$


The case $x_{*}=\left(0, a_{*}\right)$ for some $a_{*} \neq 0$ is similar. Now if $x_{*}=\left(a_{*}, c_{*}\right)$ for some $a_{*} \neq 0$ and $c_{*} \neq 0$, then we take $\delta \ll \min \left\{\left|a_{*}\right|,\left|c_{*}\right|\right\}$ and define

$$
b(x)=\frac{1}{\delta} \sum_{\epsilon_{1}, \epsilon_{2}= \pm 1} \Phi_{0}\left(\frac{x-\left(\epsilon_{1} a_{*}, \epsilon_{2} c_{*}\right)}{\delta}\right) .
$$

Easy to check that in all cases the function $b(x)$ defined above is even in $x_{1}, x_{2}$, i.e.

$$
b\left(x_{1}, x_{2}\right)=b\left(-x_{1}, x_{2}\right)=b\left(x_{1},-x_{2}\right), \quad \forall x=\left(x_{1}, x_{2}\right) \in \mathbb{R}^{2} .
$$

Now introduce the perturbation

$$
\beta(x)=\frac{1}{10 k} \sin \left(k x_{1}\right) \cdot b(x) \cdot \frac{1}{M^{\frac{1}{2}}},
$$

and define

$$
\tilde{\omega}_{0}(x)=\omega_{0}(x)+\beta(x) .
$$

We now show that if the parameter $k>0$ is taken sufficiently large then the corresponding solution $\tilde{\omega}$ will satisfy all the requirements. In the rest of this proof, to simplify the presentation, we shall use the notation $X=O\left(\frac{1}{k^{\alpha}}\right)$ if the quantity $X$ obeys the bound $X \leq C_{1} \cdot \frac{1}{k^{\alpha}}$ and the constant $C_{1}$ can depend on $\left(\omega, M, \Phi_{0}, \delta, \phi, R_{0}\right)$.

We first check (4.2)- 4.5 .

Obviously by (4.14), if $k$ is sufficiently large, then

$$
\|\beta\|_{L^{1}} \leq \frac{1}{k} \cdot \frac{1}{\sqrt{M}}\|b\|_{L^{1}} \leq\left\|\omega_{0}\right\|_{L^{1}}
$$

Similarly we can take $k$ large such that

$$
\|\beta\|_{L^{\infty}} \leq\left\|\omega_{0}\right\|_{L^{\infty}} .
$$

For the $\dot{H}^{-1}$-norm, note that $\beta$ is an odd function and $\hat{\beta}(0)=0$. Thus

$$
\begin{aligned}
\left\||\nabla|^{-1} \beta\right\|_{2} & \lesssim\|\widehat{x \beta}\|_{2}+\|\beta\|_{2} \\
& =O\left(k^{-1}\right) \leq\left\|\omega_{0}\right\|_{\dot{H}^{-1}}
\end{aligned}
$$

if $k$ is taken sufficiently large.

For the $\dot{H}^{1}$-norm, by (4.13) we have

$$
\begin{aligned}
\|\nabla \beta\|_{L^{2}}^{2} & \leq O\left(\frac{1}{k^{2}}\right)+\frac{1}{M} \cdot 10^{-2} \int b^{2}(x) \cos ^{2} k x_{1} d x \\
& \leq O\left(\frac{1}{k^{2}}\right)+\frac{1}{2 M} \cdot 10^{-2} \int b^{2}(x) d x \\
& \leq O\left(\frac{1}{k^{2}}\right)+\frac{1}{2 M} \cdot 10^{-2} \cdot 4 \cdot 4 \pi<\frac{1}{M},
\end{aligned}
$$

where we again take $k$ sufficiently large. Consequently the bound (4.5) follows. It is also not difficult to check that (4.7) can be fulfilled by taking $k$ large.

It remains to show (4.6). We shall proceed in several steps.

First we shall show

$$
\max _{0 \leq t \leq 1}\|\nabla \tilde{\omega}(t)\|_{L^{4}} \lesssim 1
$$

Here the implied constant is independent of $k$ (but is allowed to depend on other parameters). 
By a standard energy estimate, we have

$$
\begin{aligned}
\frac{d}{d t}\left(\|\nabla \tilde{\omega}(t)\|_{4}^{4}\right) & \lesssim\left\|\mathcal{R}_{i j} \tilde{\omega}(t)\right\|_{\infty} \cdot\|\nabla \tilde{\omega}(t)\|_{4}^{4} \\
& \lesssim \log \left(10+\|\tilde{\omega}\|_{2}^{2}+\|\nabla \tilde{\omega}\|_{4}^{4}\right) \cdot\|\nabla \tilde{\omega}\|_{4}^{4} .
\end{aligned}
$$

A Gronwall in time argument then yields (4.16) (by (4.14), it is easy to check that the initial data $\tilde{\omega}_{0}$ satisfies (4.16)).

Set $\eta=\omega-\tilde{\omega}$. Then

$$
\partial_{t} \eta+\Delta^{-1} \nabla^{\perp} \omega \cdot \nabla \eta+\Delta^{-1} \nabla^{\perp} \eta \cdot \nabla \tilde{\omega}=0 .
$$

Therefore noting that $\operatorname{supp}(\eta(t)) \subset B\left(z_{0}, R_{0}\right)$ for any $0 \leq t \leq 1$, we have

$$
\begin{aligned}
\frac{d}{d t}\left(\|\eta\|_{2}^{2}\right) & \lesssim\left\|\Delta^{-1} \nabla^{\perp} \eta\right\|_{4} \cdot\|\nabla \tilde{\omega}\|_{4} \cdot\|\eta\|_{2} \\
& \lesssim\|\eta\|_{2}^{2} \cdot\|\nabla \tilde{\omega}\|_{4} .
\end{aligned}
$$

Integrating in time then gives

$$
\max _{0 \leq t \leq 1}\|\eta(t)\|_{2}=O\left(k^{-1}\right) .
$$

Interpolating the bound (4.17) with (4.16) (note that $\omega$ also satisfies the same bound (4.16)), we obtain

$$
\max _{0 \leq t \leq 1}\left\|D \Delta^{-1} \nabla^{\perp}(\tilde{\omega}(t)-\omega(t))\right\|_{\infty}+\max _{0 \leq t \leq 1}\left\|\Delta^{-1} \nabla^{\perp}(\tilde{\omega}(t)-\omega(t))\right\|_{\infty}=O\left(\frac{1}{k^{\alpha}}\right),
$$

where $\alpha>0$ is some absolute constant.

Denote the forward characteristic lines associated with $\tilde{\omega}$ as $\tilde{\phi}(t, x)$ which solves

$$
\left\{\begin{array}{l}
\partial_{t} \tilde{\phi}(t, x)=\left(\Delta^{-1} \nabla^{\perp} \tilde{\omega}\right)(t, \tilde{\phi}(t, x)), \\
\tilde{\phi}(0, x)=x .
\end{array}\right.
$$

By Lemma 4.1 and (4.18), we have

$$
\max _{0 \leq t \leq 1}\left(\|\tilde{\phi}(t, \cdot)-\phi(t, \cdot)\|_{\infty}+\|(D \phi)(t, \cdot)-(D \tilde{\phi})(t, \cdot)\|_{\infty}\right)=O\left(\frac{1}{k^{\alpha}}\right) .
$$

Write $\tilde{\phi}(t, x)=\left(\tilde{\phi}_{1}(t, x), \tilde{\phi}_{2}(t, x)\right)$. By (4.11), we get

$$
\begin{aligned}
\left\|\tilde{\omega}\left(t_{0}, \cdot\right)\right\|_{\dot{H}^{1}}^{2} & \geq \int\left|\left(\nabla \tilde{\omega}_{0}\right)(x) \cdot \nabla^{\perp} \tilde{\phi}_{2}\left(t_{0}, x\right)\right|^{2} d x \\
& \geq \int\left|\nabla \tilde{\omega}_{0}(x) \cdot \nabla^{\perp} \phi_{2}\left(t_{0}, x\right)\right|^{2} d x-O\left(\frac{1}{k^{\alpha}}\right) \\
& \geq \frac{1}{2} \int\left|\nabla \beta(x) \cdot \nabla^{\perp} \phi_{2}\left(t_{0}, x\right)\right|^{2} d x-\int\left|\nabla \omega_{0}(x) \cdot \nabla^{\perp} \phi_{2}\left(t_{0}, x\right)\right|^{2} d x-O\left(\frac{1}{k^{\alpha}}\right),
\end{aligned}
$$

where in the last step we used the simple inequality

$$
|a+b|^{2} \geq \frac{1}{2}|a|^{2}-|b|^{2}, \quad \forall a, b \in \mathbb{R}^{d} .
$$

Since we are in Case 2 , we have $\left\|\omega\left(t_{0}, \cdot\right)\right\|_{\dot{H}^{1}} \leq M^{\frac{1}{3}}$. By (4.11), we get

$$
\int\left|\nabla \omega_{0}(x) \cdot \nabla^{\perp} \phi_{2}\left(t_{0}, x\right)\right|^{2} d x \leq\left\|\omega\left(t_{0}, \cdot\right)\right\|_{\dot{H}^{1}}^{2} \leq M^{\frac{2}{3}} .
$$


By our choice of the function $\beta$ and (4.12), we have

$$
\begin{aligned}
& \frac{1}{2} \int_{\mathbb{R}^{2}}\left|\nabla \beta(x) \cdot \nabla^{\perp} \phi_{2}\left(t_{0}, x\right)\right|^{2} d x \\
\geq & \frac{1}{2} \int_{\mathbb{R}^{2}}\left|\frac{\cos \left(k x_{1}\right) b(x)}{10 \sqrt{M}} \cdot \frac{\partial \phi_{2}}{\partial x_{2}}\left(t_{0}, x\right)\right|^{2} d x-O\left(k^{-2}\right) \\
\geq & \frac{1}{2} 10^{-2} \cdot M \cdot \int b^{2}(x) \cos ^{2}\left(k x_{1}\right) d x-O\left(k^{-2}\right) \\
\geq & \frac{\pi}{4} \cdot 10^{-2} \cdot M-O\left(k^{-2}\right) .
\end{aligned}
$$

Plugging (4.20) and (4.21) into (4.19), we get

$$
\begin{aligned}
\left\|\tilde{\omega}\left(t_{0}, \cdot\right)\right\|_{\dot{H}^{1}}^{2} & \geq \frac{\pi}{4} 10^{-2} M-M^{\frac{2}{3}}-O\left(k^{-2}\right)-O\left(k^{-\alpha}\right) \\
& \geq 0.7 \cdot 10^{-2} M-M^{\frac{2}{3}}
\end{aligned}
$$

if $k$ is taken sufficiently large. Clearly (4.6) follows.

\section{Local to GLOBAL: GLUing the PATChes}

In this section we prove a general proposition which allows us to glue the local solutions into a global one. We begin with some auxiliary lemmas.

To state the next lemma, we need to fix a sufficiently large constant $A_{1}>1 \mathrm{such}$ that

$$
\left\|\Delta^{-1} \nabla^{\perp} f\right\|_{\infty} \leq A_{1} \cdot\left(\|f\|_{1}+\|f\|_{\infty}\right), \quad \forall f \in L^{1}\left(\mathbb{R}^{2}\right) \cap L^{\infty}\left(\mathbb{R}^{2}\right) .
$$

Note that $A_{1}$ is an absolute constant which does not depend on any parameters.

Lemma 5.1. Consider the Euler equation on $\mathbb{R}^{2}$ :

$$
\left\{\begin{array}{l}
\partial_{t} \omega+\Delta^{-1} \nabla^{\perp} \omega \cdot \nabla \omega=0, \quad 0<t \leq 1 \\
\left.\omega\right|_{t=0}=\omega_{0}=f+g
\end{array}\right.
$$

Assume $f \in H^{k} \cap L^{1}$ for some $k \geq 2, g \in H^{2} \cap L^{1}$ and

$$
\begin{aligned}
& \left\|\omega_{0}\right\|_{L^{1}}+\left\|\omega_{0}\right\|_{L^{\infty}} \leq C_{1}<\infty, \\
& d(\operatorname{supp}(f), \operatorname{supp}(g)) \geq 100 A_{1} C_{1}>0,
\end{aligned}
$$

where $A_{1}$ is the same constant as in (5.1).

Then for any $0 \leq t \leq 1$, the following hold true:

(1) The solution $\omega(t)$ to (5.2) can be decomposed as

$$
\omega(t)=\omega_{f}(t)+\omega_{g}(t)
$$

where $\omega_{f}(0)=f, \omega_{g}(0)=g$, and $($ see $(\underline{2.2})$ )

$$
\begin{aligned}
& \operatorname{supp}\left(\omega_{f}(t)\right) \subset B\left(\operatorname{supp}(f), 2 A_{1} C_{1}\right), \\
& \operatorname{supp}\left(\omega_{g}(t)\right) \subset B\left(\operatorname{supp}(g), 2 A_{1} C_{1}\right) .
\end{aligned}
$$

(2) The Sobolev norm of $\omega_{f}(t)$ can be bounded in terms of $\|f\|_{H^{k}}$ and $C_{1}$ only:

$$
\max _{0 \leq t \leq 1}\left\|\omega_{f}(t)\right\|_{H^{k}} \leq C\left(\|f\|_{H^{k}}, C_{1}\right)<\infty
$$


Proof of Lemma 5.1. By (5.3) and (5.1), we have

$$
\max _{0 \leq t \leq 1}\left\|\Delta^{-1} \nabla^{\perp} \omega(t)\right\|_{\infty} \leq A_{1} C_{1} .
$$

By the transport nature of the equation, the support of the solution $\omega(t)$ is enlarged at most a distance $A_{1} C_{1}$ from its original support in unit time. The decomposition (5.5) follows easily from this observation and (5.4). More precisely, $\omega_{f}$ and $\omega_{g}$ are solutions to the following linear equations:

$$
\begin{aligned}
& \left\{\begin{array}{l}
\partial_{t} \omega_{f}+(u(t) \cdot \nabla) \omega_{f}=0, \\
\left.\omega_{f}\right|_{t=0}=f ;
\end{array}\right. \\
& \left\{\begin{array}{l}
\partial_{t} \omega_{g}+(u(t) \cdot \nabla) \omega_{g}=0, \\
\left.\omega_{f}\right|_{t=0}=g .
\end{array}\right.
\end{aligned}
$$

Here $u=\Delta^{-1} \nabla^{\perp} \omega$. Note that $\omega_{f}(t)$ and $\omega_{g}(t)$ stay well separated for all $0 \leq t \leq 1$ :

$$
d\left(\operatorname{supp}\left(\omega_{f}(t)\right), \operatorname{supp}\left(\omega_{g}(t)\right)\right) \geq 90 A_{1} C_{1}>0 .
$$

To show (5.6), we note that the equation for $\omega_{f}(t)$ can be rewritten as

$$
\partial_{t} \omega_{f}+\Delta^{-1} \nabla^{\perp} \omega_{f} \cdot \nabla \omega_{f}+\Delta^{-1} \nabla^{\perp} \omega_{g} \cdot \nabla \omega_{f}=0 .
$$

Note that for any multi-index $\alpha$, we have

$$
\left(\Delta^{-1} \nabla^{\perp} \partial^{\alpha} \omega_{g}\right)(x)=\int_{\mathbb{R}^{2}} K(x-y)\left(\partial^{\alpha} \omega_{g}\right)(y) d y,
$$

where $K(\cdot)$ is the kernel function corresponding to the operator $\Delta^{-1} \nabla^{\perp}$.

By (5.7), for any $x \in \operatorname{supp}\left(\omega_{f}(t)\right), y \in \operatorname{supp}\left(\omega_{g}(t)\right)$, we have $|x-y| \geq 90 A_{1} C_{1}$. Therefore we can introduce a smooth cut-off function $\chi$ on the kernel $K(\cdot)$ and rewrite (5.9) as

$$
\begin{aligned}
\left(\Delta^{-1} \nabla^{\perp} \partial^{\alpha} \omega_{g}\right)(x) & =\int_{\mathbb{R}^{2}} K(x-y) \chi_{|x-y| \geq 80 A_{1} C_{1}}\left(\partial^{\alpha} \omega_{g}\right)(y) d y \\
& =\int_{\mathbb{R}^{2}}(-1)^{|\alpha|} \partial_{y}^{\alpha}\left(K(x-y) \chi_{|x-y| \geq 80 A_{1} C_{1}}\right) \omega_{g}(y) d y \\
& =\int_{\mathbb{R}^{2}} \tilde{K}_{\alpha}(x-y) \omega_{g}(y) d y
\end{aligned}
$$

where the modified kernel $\tilde{K}_{\alpha}$ satisfies

$$
\left|\tilde{K}_{\alpha}(z)\right| \lesssim_{C_{1}, \alpha}\left(1+|z|^{2}\right)^{-\frac{1}{2}}, \quad \forall z \in \mathbb{R}^{2} .
$$

By using $L^{1}$ and $L^{\infty}$ conservation, we have

$$
\left\|\omega_{f}(t)\right\|_{L^{1}}+\left\|\omega_{f}(t)\right\|_{L^{\infty}}+\left\|\omega_{g}(t)\right\|_{L^{1}}+\left\|\omega_{g}(t)\right\|_{L^{\infty}} \leq C_{1} .
$$

Therefore by (5.10), (5.11), (5.12) and the Cauchy-Schwartz inequality, we have

$$
\max _{0 \leq t \leq 1} \max _{x \in \operatorname{supp}\left(\omega_{f}(t)\right)}\left|\left(\Delta^{-1} \nabla^{\perp} \partial^{\alpha} \omega_{g}\right)(t, x)\right| \lesssim C_{1}, \alpha 1 .
$$

The estimate (5.13) shows that the drift term $\Delta^{-1} \nabla^{\perp} \omega_{g}$ in (5.8) is arbitrarily smooth on the support of $\omega_{f}$. Therefore the estimate (5.6) follows from the standard 
energy estimate. For the sake of completeness we sketch the detail here for $k=2$. By (5.8), we have

$$
\begin{aligned}
& \frac{d}{d t}\left(\left\|\Delta \omega_{f}(t)\right\|_{2}^{2}\right) \\
& \leq\left|\int_{\mathbb{R}^{2}} \Delta\left(\Delta^{-1} \nabla^{\perp} \omega_{f} \cdot \nabla \omega_{f}\right) \Delta \omega_{f} d x\right|+\left|\int_{\mathbb{R}^{2}} \Delta\left(\Delta^{-1} \nabla^{\perp} \omega_{g} \cdot \nabla \omega_{f}\right) \Delta \omega_{f} d x\right| \\
& \leq \int_{\mathbb{R}^{2}}\left|\Delta^{-1} \nabla^{\perp} \partial \omega_{f}\right| \cdot\left|\partial^{2} \omega_{f}\right| \cdot\left|\Delta \omega_{f}\right| d x+\max _{\substack{x \in \operatorname{supp}_{f}\left(\omega_{f}(t)\right) \\
|\alpha| \leq 2}}\left|\Delta^{-1} \nabla^{\perp} \partial^{\alpha} \omega_{g}(x)\right| \cdot\left\|\omega_{f}(t)\right\|_{H^{2}}^{2} \\
& \lesssim C_{1}\left(1+\left\|\mathcal{R}_{i j} \omega_{f}(t)\right\|_{\infty}\right) \cdot\left\|\omega_{f}(t)\right\|_{H^{2}}^{2},
\end{aligned}
$$

where $\mathcal{R}_{i j}$ denotes the Riesz transform. By the usual log interpolation inequality and (5.12), we have

$$
\left\|\mathcal{R}_{i j} \omega_{f}\right\|_{\infty} \lesssim C_{1} \log \left(10+\left\|\omega_{f}(t)\right\|_{H^{2}}^{2}\right)
$$

Therefore

$$
\frac{d}{d t}\left(\left\|\omega_{f}(t)\right\|_{H^{2}}^{2}\right) \lesssim C_{1} \log \left(10+\left\|\omega_{f}(t)\right\|_{H^{2}}^{2}\right) \cdot\left\|\omega_{f}(t)\right\|_{H^{2}}^{2} .
$$

A $\log$ Gronwall in time argument then yields (5.6).

Lemma 5.2. Let $\omega$ and $\tilde{\omega}$ be solutions to the Euler equations

$$
\left\{\begin{array}{l}
\partial_{t} \omega+\Delta^{-1} \nabla^{\perp} \omega \cdot \nabla \omega=0, \quad 0<t \leq 1, \\
\left.\omega\right|_{t=0}=\omega_{0}=f+g
\end{array}\right.
$$

and

$$
\left\{\begin{array}{l}
\partial_{t} \tilde{\omega}+\Delta^{-1} \nabla^{\perp} \tilde{\omega} \cdot \nabla \tilde{\omega}=0, \quad 0<t \leq 1 \\
\left.\tilde{\omega}\right|_{t=0}=f .
\end{array}\right.
$$

Assume $f \in H^{3} \cap L^{1}, g \in H^{2} \cap L^{1}$ and

$$
\left\|\omega_{0}\right\|_{L^{1}}+\left\|\omega_{0}\right\|_{L^{\infty}} \leq C_{1}<\infty .
$$

Assume also $f$ is compactly supported such that

$$
\operatorname{Leb}(\operatorname{supp}(f)) \leq C_{2}<\infty .
$$

Then for any $\epsilon>0$, there exists $R_{\epsilon}=R_{\epsilon}\left(\epsilon,\|f\|_{H^{3}}, C_{1}, C_{2}\right)>0$ such that if

$$
d(\operatorname{supp}(f), \operatorname{supp}(g)) \geq R_{\epsilon}>0,
$$

then for any $0<t \leq 1$, the following hold true:

(1) $\omega(t)$ has the decomposition

$$
\omega(t)=\omega_{f}(t)+\omega_{g}(t)
$$

where

$$
\begin{aligned}
& \operatorname{supp}\left(\omega_{f}(t)\right) \subset B\left(\operatorname{supp}(f), 2 A_{1} C_{1}\right) ; \\
& \operatorname{supp}\left(\omega_{g}(t)\right) \subset B\left(\operatorname{supp}(g), 2 A_{1} C_{1}\right) ; \\
& d\left(\operatorname{supp}\left(\omega_{f}(t), \operatorname{supp}\left(\omega_{g}(t)\right)\right) \geq 100 A_{1} C_{1} .\right.
\end{aligned}
$$

Here $A_{1}$ is the same constant in (5.1). 
(2) The support of $\tilde{\omega}(t)$ also satisfies

$$
\operatorname{supp}(\tilde{\omega}(t)) \subset B\left(\operatorname{supp}(f), 2 A_{1} C_{1}\right) .
$$

(3) $\omega_{f}(t)$ and $\tilde{\omega}(t)$ are close:

$$
\max _{0 \leq t \leq 1}\left\|\omega_{f}(t)-\tilde{\omega}(t)\right\|_{H^{2}}<\epsilon .
$$

Proof of Lemma 5.2. Note that (5.15) and (5.17) follows directly from Lemma 5.1 we just need to take $R_{\epsilon} \geq 100 A_{1} C_{1}$. By Lemma 5.1 we have

$$
\begin{aligned}
& \max _{0 \leq t \leq 1}\left\|\omega_{f}(t)-\tilde{\omega}(t)\right\|_{H^{3}} \\
\leq & \max _{0 \leq t \leq 1}\left\|\omega_{f}(t)\right\|_{H^{3}}+\max _{0 \leq t \leq 1}\|\tilde{\omega}(t)\|_{H^{3}} \\
\leq & C_{3}=C_{3}\left(\|f\|_{H^{3}}, C_{1}\right) .
\end{aligned}
$$

Set $\eta(t)=\omega_{f}(t)-\tilde{\omega}(t)$. Then by (5.8), we have

$$
\left\{\begin{array}{l}
\partial_{t} \eta+\Delta^{-1} \nabla^{\perp} \eta \cdot \nabla \omega_{f}+\Delta^{-1} \nabla^{\perp} \tilde{\omega} \cdot \nabla \eta+\Delta^{-1} \nabla^{\perp} \omega_{g} \cdot \nabla \omega_{f}=0, \quad 0<t \leq 1, \\
\eta(0)=0 .
\end{array}\right.
$$

For $x \in \operatorname{supp}\left(\omega_{f}(t)\right)$, we have

$$
\begin{aligned}
\left|\left(\Delta^{-1} \nabla^{\perp} \omega_{g}\right)(t, x)\right| & \lesssim \int_{|x-y| \geq \frac{1}{2} R_{\epsilon}} \frac{1}{|x-y|}\left|\omega_{g}(t, y)\right| d y \\
& \lesssim R_{\epsilon}^{-\frac{1}{2}} \cdot\left\|\omega_{g}\right\|_{\frac{4}{3}} \lesssim R_{\epsilon}^{-\frac{1}{2}} \cdot C_{1} .
\end{aligned}
$$

Therefore

$$
\begin{aligned}
& \frac{d}{d t}\left(\|\eta(t)\|_{2}^{2}\right) \\
\lesssim & \left\|\Delta^{-1} \nabla^{\perp} \eta\right\|_{3} \cdot\|\eta\|_{2} \cdot\left\|\nabla \omega_{f}\right\|_{6}+R_{\epsilon}^{-\frac{1}{2}} \cdot C_{1} \cdot\left\|\nabla \omega_{f}\right\|_{2} \cdot\|\eta\|_{2} .
\end{aligned}
$$

By Sobolev embedding, (5.14), (5.16), (5.17) and Hölder, we have

$$
\begin{aligned}
\left\|\Delta^{-1} \nabla^{\perp} \eta\right\|_{3} & \lesssim\|\eta\|_{\frac{6}{5}} \\
& \lesssim C_{1}, C_{2}\|\eta\|_{2} .
\end{aligned}
$$

By (5.19) and Sobolev embedding we have

$$
\left\|\nabla \omega_{f}\right\|_{6} \lesssim C_{3}
$$

Therefore integrating (5.20) in time, we obtain for some $C_{4}=C_{4}\left(C_{1}, C_{2}, C_{3}\right)>0$ that

$$
\max _{0 \leq t \leq 1}\|\eta(t)\|_{2} \leq R_{\epsilon}^{-\frac{1}{2}} \cdot C_{4}
$$

The desired estimate (5.18) follows easily from interpolating (5.21), (5.19) and taking $R_{\epsilon}$ sufficiently large.

Proposition 5.3 (Almost non-interacting patches). Let $\left\{\omega_{j}\right\}_{j=1}^{\infty}$ be a sequence of functions in $C_{c}^{\infty}(B(0,1))$ and satisfy the following condition:

$$
\sum_{j=1}^{\infty}\left\|\omega_{j}\right\|_{H^{1}}^{2}+\sum_{j=1}^{\infty}\left\|\omega_{j}\right\|_{L^{1}}+\sup _{j}\left\|\omega_{j}\right\|_{L^{\infty}} \leq C_{1}<\infty
$$

Here we may assume $C_{1}>1$. 
Then there exist centers $x_{j} \in \mathbb{R}^{2}$ whose mutual distance are sufficiently large (i.e. $\left|x_{j}-x_{k}\right| \gg 1$ if $j \neq k$ ) such that the following hold:

(1) Take the initial data

$$
\omega_{0}(x)=\sum_{j=1}^{\infty} \omega_{j}\left(x-x_{j}\right),
$$

then $\omega_{0} \in L^{1} \cap L^{\infty} \cap H^{1} \cap C^{\infty}$. Furthermore for any $j \neq k$

$$
B\left(x_{j}, 100 A_{1} C_{1}\right) \cap B\left(x_{k}, 100 A_{1} C_{1}\right)=\varnothing .
$$

Here $A_{1}$ is the same absolute constant as in (5.1).

(2) With $\omega_{0}$ as initial data, there exists a unique solution $\omega$ to the Euler equation

$$
\partial_{t} \omega+\Delta^{-1} \nabla^{\perp} \omega \cdot \nabla \omega=0
$$

on the time interval $[0,1]$ satisfying $\omega \in L^{1} \cap L^{\infty} \cap C^{\infty}, u=\Delta^{-1} \nabla^{\perp} \omega \in C^{\infty}$. Moreover for any $0 \leq t \leq 1$,

$$
\operatorname{supp}(\omega(t, \cdot)) \subset \bigcup_{j=1}^{\infty} B\left(x_{j}, 3 A_{1} C_{1}\right) .
$$

(3) For any $\epsilon>0$, there exists an integer $J_{\epsilon}$ sufficiently large such that if $j \geq J_{\epsilon}$, then

$$
\max _{0 \leq t \leq 1}\left\|\omega(t, \cdot)-\tilde{\omega}_{j}(t, \cdot)\right\|_{H^{2}\left(B\left(x_{j}, 3 A_{1} C_{1}\right)\right)}<\epsilon .
$$

Here $\tilde{\omega}_{j}$ is the solution solving the equation

$$
\left\{\begin{array}{l}
\partial_{t} \tilde{\omega}_{j}+\Delta^{-1} \nabla+\tilde{\omega}_{j} \cdot \nabla \tilde{\omega}_{j}=0, \quad 0<t \leq 1, x \in \mathbb{R}^{2} ; \\
\tilde{\omega}_{j}(t=0, x)=\omega_{j}\left(x-x_{j}\right), \quad x \in \mathbb{R}^{2} .
\end{array}\right.
$$

Proof of Proposition 5.3 .

Step 1. Choice of the centers $x_{j}$.

For each $\omega_{j}, j \geq 1$, we choose $R_{j}=R_{j}\left(\left\|\omega_{j}\right\|_{H^{3}}, C_{1}\right)>0$ corresponding to $f=\omega_{j}$ and $\epsilon=2^{-j}$ in Lemma $5.2\left(C_{1}\right.$ is the same constant as in (5.22) $)$. More precisely, if we take

$$
\left\{\begin{array}{l}
\partial_{t} \omega+\Delta^{-1} \nabla^{\perp} \omega \cdot \nabla \omega=0, \quad 0<t \leq 1, \\
\left.\omega\right|_{t=0}=\omega_{j}+g,
\end{array}\right.
$$

and

$$
\left\{\begin{array}{l}
\partial_{t} \tilde{\omega}+\Delta^{-1} \nabla^{\perp} \tilde{\omega} \cdot \nabla \tilde{\omega}=0 \\
\left.\tilde{\omega}\right|_{t=0}=\omega_{j}
\end{array}\right.
$$

with

$$
\left\|\omega_{j}+g\right\|_{L^{1}}+\left\|\omega_{j}+g\right\|_{L^{\infty}} \leq C_{1}<\infty,
$$

and

$$
d\left(\operatorname{supp}\left(\omega_{j}\right), \operatorname{supp}(g)\right) \geq R_{j},
$$

then $\omega(t)=\omega_{f}(t)+\omega_{g}(t)$ with 


$$
\operatorname{supp}\left(\omega_{f}(t)\right) \subset B\left(0,1+2 A_{1} C_{1}\right)
$$

and

$$
\max _{0 \leq t \leq 1}\left\|\omega_{f}(t)-\tilde{\omega}(t)\right\|_{H^{2}}<2^{-j}
$$

With the numbers $R_{j}$ properly defined, we now describe how to choose the centers $x_{j}$ inductively. First set $x_{1}=0$. For $j \geq 2$, assume $x_{1}, \cdots, x_{j-1}$ have already been chosen. Let

$$
f_{j-1}(x)=\sum_{l=1}^{j-1} \omega_{l}\left(x-x_{l}\right)
$$

and consider the problems

$$
\left\{\begin{array}{l}
\partial_{t} \omega+\Delta^{-1} \nabla^{\perp} \omega \cdot \nabla \omega=0, \quad 0<t \leq 1 \\
\left.\omega\right|_{t=0}=f_{j-1}+g
\end{array}\right.
$$

and

$$
\left\{\begin{array}{l}
\partial_{t} \tilde{\omega}+\Delta^{-1} \nabla^{\perp} \tilde{\omega} \cdot \nabla \tilde{\omega}=0 \\
\left.\tilde{\omega}\right|_{t=0}=f_{j-1}
\end{array}\right.
$$

with

$$
\left\|f_{j-1}+g\right\|_{L^{1}}+\left\|f_{j-1}+g\right\|_{L^{\infty}} \leq C_{1}<\infty .
$$

By Lemma 5.2 we can find $\tilde{R}_{j}=\tilde{R}_{j}\left(\left\|f_{j-1}\right\|_{H^{3}}, C_{1}\right)>0$ such that if

$$
d\left(\operatorname{supp}\left(f_{j-1}\right), \operatorname{supp}(g)\right)>\tilde{R}_{j},
$$

then

$$
\max _{0 \leq t \leq 1}\left\|\omega_{f_{j-1}}(t)-\tilde{\omega}(t)\right\|_{H^{2}}<2^{-j}
$$

We now choose $x_{j}$ such that

$$
d\left(\operatorname{supp}\left(f_{j-1}\right), x_{j}\right)>2 \tilde{R}_{j}+2 \sum_{l=1}^{j} R_{l}+1000 A_{1} C_{1}+10^{j} .
$$

By induction it is easy to verify that (5.23) holds.

Step 2. Construction of the solution $\omega(t)$ by patching.

Since $\omega_{0} \in L^{1} \cap L^{\infty}$, the usual Yudovich theory already gives existence and uniqueness of a weak solution in $L^{1} \cap L^{\infty}$. Here thanks to the special type of initial data we shall give a more direct construction which also yields the regularity of the solution at one stroke.

To this end, denote for each $m \geq 2$

$$
\omega_{0}^{(m)}(x)=\sum_{j=1}^{m} \omega_{j}\left(x-x_{j}\right)
$$


and let $\omega^{(m)}(t, x)$ be the corresponding solution to the Euler equation. Obviously for $0 \leq t \leq 1$

$$
\operatorname{supp}\left(\omega^{(m)}(t)\right) \subset \bigcup_{j=1}^{m} B\left(x_{j}, 3 A_{1} C_{1}\right)
$$

Now we define $\omega(t, x)$ as follows

$$
\omega(t, x)=\left\{\begin{array}{l}
\lim _{m \rightarrow \infty} \omega^{(m)}(t, x), \quad \text { if } x \in \bigcup_{j=1}^{\infty} B\left(x_{j}, 3 A_{1} C_{1}\right) \\
0, \quad \text { otherwise }
\end{array}\right.
$$

We now justify that $\omega(t, x)$ is well-defined and is the desired solution.

Fix $j_{0} \geq 1$ and consider the ball $B\left(x_{j_{0}}, 3 A_{1} C_{1}\right)$. By (5.29) (setting $\omega=\omega^{(m)}$ and $\left.\tilde{\omega}=\omega^{(m-1)}\right)$, we have

$$
\max _{0 \leq t \leq 1}\left\|\omega^{(m)}(t)-\omega^{(m-1)}(t)\right\|_{H^{2}\left(B\left(x_{j_{0}}, 3 A_{1} C_{1}\right)\right)} \leq 2^{-m}, \quad \text { if } m \geq j_{0}+1 .
$$

By Lemma 5.1, we also have for any $k \geq 3$,

$$
\max _{0 \leq t \leq 1}\left\|\omega^{(m)}(t)\right\|_{H^{k}\left(B\left(x_{j_{0}}, 3 A_{1} C_{1}\right)\right)} \leq C_{k}=C_{k}\left(\left\|\omega_{j_{0}}\right\|_{H^{k}}, C_{1}\right), \quad \text { if } m \geq j_{0}+1 .
$$

Thus $\left(\omega^{(m)}\right)$ forms a Cauchy sequence in $H^{k}\left(B\left(x_{j_{0}}, 3 A_{1} C_{1}\right)\right)$ for any $k \geq 2$ and hence converge to a unique limit $\omega(t, x) \in C^{\infty}\left(B\left(x_{j_{0}}, 3 A_{1} C_{1}\right)\right)$. Clearly (5.24) holds. Easy to check $\omega \in L^{\infty}$.

By using the Lebesgue Dominated Convergence Theorem, we have

$$
\|\omega(t)\|_{L^{1}\left(B\left(x_{j_{0}}, 3 A_{1} C_{1}\right)\right)} \leq \lim _{m \rightarrow \infty}\left\|\omega^{(m)}(t)\right\|_{L^{1}\left(B\left(x_{j_{0}}, 3 A_{1} C_{1}\right)\right)}=\left\|\omega_{j_{0}}\right\|_{L^{1}} .
$$

Summing in $j_{0}$ then gives us $\omega \in L^{1}$.

We now show that $\Delta^{-1} \nabla^{\perp} \omega^{(m)}$ converges locally uniformly to $\Delta^{-1} \nabla^{\perp} \omega$ on $\bigcup_{j=1}^{\infty} B\left(x_{j}, 3 A_{1} C_{1}\right)$. By construction we can decompose

$$
\omega^{(m)}(t, x)=\sum_{j=1}^{m} \omega_{j}^{(m)}(t, x)
$$

where

$$
\operatorname{supp}\left(\omega_{j}^{(m)}\right) \subset B\left(x_{j}, 3 A_{1} C_{1}\right)
$$

Also we have

$$
\omega(t, x)=\sum_{j=1}^{\infty} \omega_{j}^{(\infty)}(t, x), \operatorname{supp}\left(\omega_{j}^{(\infty)}\right) \subset B\left(x_{j}, 3 A_{1} C_{1}\right) .
$$

The summation above is actually a finite sum since for each $x$ there exists at most one $j$ such that $\omega_{j}^{(\infty)}(t, x) \neq 0$. 
Now fix $j_{0} \geq 1$. Then for $x \in B\left(x_{j_{0}}, 2 A_{1} C_{1}\right)$ and $m \geq j_{0}+1$, we have

$$
\begin{aligned}
\left|\left(\Delta^{-1} \nabla^{\perp} \omega^{(m)}\right)(x)-\left(\Delta^{-1} \nabla^{\perp} \omega\right)(x)\right| \leq & \mid\left(\Delta^{-1} \nabla^{\perp}\left(\omega_{j_{0}}^{(m)}-\omega_{j_{0}}^{(\infty)}\right)(x) \mid\right. \\
& +\sum_{\substack{j=1 \\
j \neq j_{0}}}^{m}\left|\left(\Delta^{-1} \nabla^{\perp}\left(\omega_{j}^{(m)}-\omega_{j}^{(\infty)}\right)\right)(x)\right| \\
& +\sum_{j=m+1}^{\infty}\left|\left(\Delta^{-1} \nabla^{\perp} \omega_{j}^{(\infty)}\right)(x)\right| .
\end{aligned}
$$

For (5.32), we use the inequality (5.1) to get

$$
\begin{aligned}
\|\left(\Delta^{-1} \nabla^{\perp}\left(\omega_{j_{0}}^{(m)}-\omega_{j_{0}}^{(\infty)}\right)(x) \|_{\infty}\right. & \leq A_{1}\left(\left\|\omega_{j_{0}}^{(m)}-\omega_{j_{0}}^{(\infty)}\right\|_{1}+\left\|\omega_{j_{0}}^{(m)}-\omega_{j_{0}}^{(\infty)}\right\|_{\infty}\right) \\
& \lesssim C_{1}\left\|\omega_{j_{0}}^{(m)}-\omega_{j_{0}}^{(\infty)}\right\|_{\infty} \\
& \lesssim C_{1}\left\|\omega^{(m)}-\omega\right\|_{L^{\infty}\left(B\left(x_{j_{0}}, 3 A_{1} C_{1}\right)\right.} \\
& \rightarrow 0, \quad \text { as } m \rightarrow \infty,
\end{aligned}
$$

since $\omega^{(m)}$ converges uniformly to $\omega$ on the ball $B\left(x_{j_{0}}, 3 A_{1} C_{1}\right)$.

For (5.33), note that for $j \neq j_{0}$ (see (5.30)

$$
d\left(\operatorname{supp}\left(\omega_{j}^{(m)}-\omega_{j}^{(\infty)}\right), B\left(x_{j_{0}}, 3 A_{1} C_{1}\right)\right) \geq 2^{j} .
$$

Therefore by using an estimate similar to (5.10), we have

$$
\begin{aligned}
\text { (5.33) } & \lesssim \sum_{\substack{j=1 \\
j \neq j_{0}}}^{\infty} 2^{-j}\left\|\omega_{j}^{(m)}-\omega_{j}^{(\infty)}\right\|_{L^{1} \cap L^{\infty}} \\
& \lesssim C_{1} \sum_{j=1}^{\infty} 2^{-j}\left\|\omega^{(m)}-\omega\right\|_{L^{\infty}\left(B\left(x_{j}, 3 A_{1} C_{1}\right)\right.} \\
& \rightarrow 0, \quad \text { as } m \rightarrow \infty .
\end{aligned}
$$

Similarly

$$
\text { (5.34) } \lesssim_{C_{1}} \sum_{j=m+1}^{\infty} 2^{-j} \rightarrow 0, \quad \text { as } m \rightarrow \infty .
$$

Hence we have shown that $\Delta^{-1} \nabla^{\perp} \omega^{(m)} \rightarrow \Delta^{-1} \nabla^{\perp} \omega$ locally uniformly on compact sets (and also uniformly in $t$ ) as $m$ tends to infinity. By writing

$$
\omega^{(m)}(t)=\omega^{(m)}(0)+\int_{0}^{t}\left(\Delta^{-1} \nabla^{\perp} \omega^{(m)} \cdot \nabla \omega^{(m)}\right)(\tau) d \tau,
$$

and sending $m$ to infinity, we conclude that $\omega$ is the desired solution on the time interval $[0,1]$.

Finally (5.25) is a simple consequence of Lemma 5.2 and our choice of the centers $x_{j}($ see (5.27) $)$.

We are now ready to complete the 
Proof of Theorem 1.2. For each $j \geq 2$, we choose (by a slight abuse of notation) $h_{j}=h_{A_{j}}$ according to (3.4) with the parameter $A_{j}$ to be taken sufficiently large. Consider the Euler equation

$$
\left\{\begin{array}{l}
\partial_{t} \omega+\Delta^{-1} \nabla^{\perp} \omega \cdot \nabla \omega=0, \quad 0<t \leq 1, \\
\left.\omega\right|_{t=0}=h_{j} .
\end{array}\right.
$$

By Proposition 3.5, we obtain for some $t_{j} \in\left(0, \frac{1}{\log \log A_{j}}\right)$,

$$
\left\|(D \phi)\left(t_{j}, \cdot\right)\right\|_{\infty}>\log \log A_{j}
$$

where $\phi$ is defined in (3.15).

We then use Proposition 4.2 to find $\tilde{\omega}_{j}^{(0)} \in C_{c}^{\infty}(B(0,1)), \tilde{\omega}_{j}^{(0)}$ odd in both $x_{1}$ and $x_{2}$, such that

$$
\begin{aligned}
& \left\|\tilde{\omega}_{j}^{(0)}\right\|_{L^{1}} \leq 2\left\|h_{j}\right\|_{L^{1}}, \\
& \left\|\tilde{\omega}_{j}^{(0)}\right\|_{L^{\infty}} \leq 2\left\|h_{j}\right\|_{L^{\infty}}, \\
& \left\|\tilde{\omega}_{j}^{(0)}\right\|_{\dot{H}^{1}} \leq\left\|h_{j}\right\|_{\dot{H}^{1}}+2^{-j}, \\
& \left\|\tilde{\omega}_{j}^{(0)}\right\|_{\dot{H}^{-1}} \leq 2\left\|h_{j}\right\|_{\dot{H}^{-1}}, \\
& \left\|\tilde{\omega}_{j}\left(t_{j}, \cdot\right)\right\|_{\dot{H}^{1}}>j,
\end{aligned}
$$

where $\tilde{\omega}_{j}(t)$ is the solution to the Euler equation

$$
\left\{\begin{array}{l}
\partial_{t} \tilde{\omega}_{j}+\Delta^{-1} \nabla^{\perp} \tilde{\omega}_{j} \cdot \nabla \tilde{\omega}_{j}=0, \quad 0<t \leq 1, \\
\left.\tilde{\omega}_{j}\right|_{t=0}=\tilde{\omega}_{j}^{(0)} .
\end{array}\right.
$$

We then apply Proposition 5.3 to $\omega_{1}=\omega_{0}^{(p)}, \omega_{j}=\tilde{\omega}_{j}^{(0)}$ for $j \geq 2$ and find the centers $x_{j}$. Obviously by (5.35) and (5.25), we have

$$
\operatorname{ess}^{-\sup _{0<t \leq t_{0}}}\|\omega(t, \cdot)\|_{\dot{H}^{1}}=+\infty, \quad \forall 0<t_{0} \leq 1 .
$$

It is not difficult to check the $\dot{H}^{-1}$ regularity of the constructed solution since on each patch the $L^{2}$ norm of the velocity field is preserved. The theorem is proved.

\section{The 2D COMPACTLY SUPPORTED CASE}

Lemma 6.1 (Control of the support). Suppose $\omega=\omega(t, x)$ is a smooth solution to the following equation:

$$
\left\{\begin{array}{l}
\partial_{t} \omega+\Delta^{-1} \nabla^{\perp} \omega \cdot \nabla \omega+\left(b_{1}+b_{2}\right) \cdot \nabla \omega=0, \\
\left.\omega\right|_{t=0}=f,
\end{array}\right.
$$

where $b_{1}=b_{1}(t, x), b_{2}=b_{2}(t, x), f=f(x)$ are smooth functions satisfying the following conditions:

- $\|f\|_{\infty} \leq C_{f}$ for some constant $C_{f}>0$, and

$$
\operatorname{supp}(f) \subset B(0, R), \quad R>0 .
$$

- $b_{1}, b_{2}$ are incompressible, i.e. $\nabla \cdot b_{1}=\nabla \cdot b_{2}=0$.

- For some $B_{1}>0$,

$$
\left|b_{1}(t, x)\right| \leq B_{1}|x|, \quad \forall x \in \mathbb{R}^{2} .
$$


- For some $B_{2}>0$,

$$
\left|b_{2}(t, x)\right| \leq B_{2}|x|^{2}, \quad \forall x \in \mathbb{R}^{2} .
$$

Then there exists $R_{0}=R_{0}\left(C_{f}, B_{1}, B_{2}\right)>0, t_{0}=t_{0}\left(C_{f}, B_{1}, B_{2}\right)>0$, such that if $0<R \leq R_{0}$, then

$$
\operatorname{supp}(\omega(t, \cdot)) \subset B(0,2 R), \quad \forall 0 \leq t \leq t_{0} .
$$

Proof of Lemma 6.1. Define the forward characteristic lines $\phi=\phi(t, x)$ which solves the ODE

$$
\left\{\begin{array}{l}
\partial_{t} \phi(t, x)=\left(\Delta^{-1} \nabla^{\perp} \omega+b_{1}+b_{2}\right)(t, \phi(t, x)), \\
\phi(t=0, x)=x, \quad x \in \mathbb{R}^{2} .
\end{array}\right.
$$

By using the assumptions, we compute

$$
\begin{aligned}
& \frac{d}{d t}\left(|\phi(t, x)|^{2}\right) \\
\leq & \left\|\left(\Delta^{-1} \nabla^{\perp} \omega\right)(t, \cdot)\right\|_{\infty}|\phi(t, x)|+B_{1}|\phi(t, x)|^{2}+B_{2}|\phi(t, x)|^{3} .
\end{aligned}
$$

Since both $b_{1}$ and $b_{2}$ are incompressible, we have

$$
\|\omega(t, \cdot)\|_{L^{1}}=\|\omega(t=0, \cdot)\|_{L^{1}}=\|f\|_{L^{1}} \leq C_{f} \cdot \pi R^{2} .
$$

Then by interpolation and $L^{\infty}$ conservation, we get

$$
\begin{aligned}
\left\|\left(\Delta^{-1} \nabla^{\perp} \omega\right)(t, \cdot)\right\|_{\infty} & \lesssim\|\omega(t, \cdot)\|_{L^{1}}^{\frac{1}{2}}\|\omega(t, \cdot)\|_{L^{\infty}}^{\frac{1}{2}} \\
& \lesssim\|f\|_{L^{1}}^{\frac{1}{2}}\|f\|_{L^{\infty}}^{\frac{1}{2}} \\
& \lesssim C_{f} R,
\end{aligned}
$$

where in the last inequality we have used (6.2) and all the implied constants are absolute constants.

Plugging (6.3) into (6.1), we obtain

$$
\frac{d}{d t}(|\phi(t, x)|) \lesssim C_{f} R+B_{1}|\phi(t, x)|+B_{2}|\phi(t, x)|^{2} .
$$

The desired result then follows from time integration and choosing $R_{0}, t_{0}$ sufficiently small.

For the compactly supported case, we need to use a slight variant of the function $h_{A}$ defined in (3.4). We now take any $A \gg 1$ and

$$
g_{A}(x)=\frac{1}{\log \log \log \log A} \cdot \frac{1}{\sqrt{\log A}} \sum_{A \leq k \leq A+\log A} \eta_{k}(x),
$$

where $\eta_{k}$ was defined in (3.1).

It is easy to check that

$$
\begin{aligned}
& \operatorname{supp}\left(g_{A}\right) \subset B\left(0, R_{A}\right), \quad \text { with } R_{A} \sim 2^{-A}, \\
&\left\|g_{A}\right\|_{H^{1}} \lesssim \frac{1}{\log \log \log \log A}, \\
&\left\|g_{A}\right\|_{L^{\infty}} \lesssim \frac{1}{\sqrt{\log A}}, \\
&\left\|D^{2} g_{A}\right\|_{L^{\infty}} \lesssim 2^{2(A+\log A)} .
\end{aligned}
$$


The main difference between $g_{A}$ and $h_{A}$ is that the former has weaker dependence on $A$ in terms of the bounds on higher derivatives. This fact will be used in the perturbation theory later (see Lemma 6.3).

The following is a variant of Proposition 3.5. Note that the additional drift term has a special form which makes the class of odd flows invariant.

Lemma 6.2. Let $\omega=\omega(t, x)$ be the smooth solution to the equation

$$
\left\{\begin{array}{l}
\partial_{t} \omega+\Delta^{-1} \nabla^{\perp} \omega \cdot \nabla \omega+b \cdot \nabla \omega=0, \\
\left.\omega\right|_{t=0}=g_{A}
\end{array}\right.
$$

where $g_{A}$ is defined in (6.4), $b=b(t, x)$ takes the form

$$
b(t, x)=b_{0}(t)\left(\begin{array}{c}
-x_{1} \\
x_{2}
\end{array}\right), \quad x \in \mathbb{R}^{2} ;
$$

and $b_{0}(t)$ is a smooth function satisfying

$$
\left\|b_{0}\right\|_{\infty} \leq B_{0}<\infty .
$$

Let $\phi=\phi(t, x)$ be the associated forward characteristic line which solves

$$
\left\{\begin{array}{l}
\partial_{t} \phi(t, x)=\left(\Delta^{-1} \nabla^{\perp} \omega+b\right)(t, \phi(t, x)) \\
\phi(t=0, x)=x, \quad x \in \mathbb{R}^{2}
\end{array}\right.
$$

Then there exists $A_{0}=A_{0}\left(B_{0}\right)>0$ such that if $A>A_{0}$, then

$$
\max _{0 \leq t \leq \frac{1}{\log \log A}}\|(D \phi)(t, \cdot)\|_{\infty}>\log \log A .
$$

Proof of Lemma 6.2. Thanks to the special assumption (6.5), it is easy to check that $\omega(t, x)$ is still an odd function in $x_{1}$ and $x_{2}$ for any $t$. We can then repeat the proof of Proposition 3.5 or use the simplified version as in the proof of Proposition 3.6. We omit the details.

The next lemma shows that the patch dynamics can still be controlled under a suitable perturbation in the drift term. This will play an important role in our later constructions. Since we no longer have odd symmetry at our disposal, we need to carry out a perturbative analysis.

Lemma 6.3. Let $W=W(t, x)$ be a smooth solution to the equation

$$
\left\{\begin{array}{l}
\partial_{t} W+\Delta^{-1} \nabla^{\perp} W \cdot \nabla W+(b(t, x)+r(t, x)) \cdot \nabla W=0, \\
\left.W\right|_{t=0}=W_{0}=g_{A},
\end{array}\right.
$$

where the functions $g_{A}, b, r$ satisfies the following conditions:

- $g_{A}$ is the same as defined in (6.4);

- $b(t, x)=b_{0}(t)\left(\begin{array}{c}-x_{1} \\ x_{2}\end{array}\right), \quad\left\|b_{0}\right\|_{\infty} \leq B_{0}<\infty$;

- $r$ is incompressible and

$$
\begin{aligned}
& |r(t, x)| \leq B_{1} \cdot|x|^{2}, \\
& |(D r)(t, x)| \leq B_{1} \cdot|x|, \\
& \left|\left(D^{2} r\right)(t, x)\right| \leq B_{1}, \quad \forall x \in \mathbb{R}^{2}, 0 \leq t \leq 1 .
\end{aligned}
$$

Here $B_{1}>0$ is a constant. 
Let $\Phi=\Phi(t, x)$ be the characteristic line which solves the $O D E$

$$
\left\{\begin{array}{l}
\partial_{t} \Phi(t, x)=\left(\Delta^{-1} \nabla^{\perp} W+b+r\right)(t, \Phi(t, x)), \\
\Phi(t=0, x)=x, \quad x \in \mathbb{R}^{2} .
\end{array}\right.
$$

Then there exists $A_{0}=A_{0}\left(B_{0}, B_{1}\right)>0$ such that if $A>A_{0}$, then

$$
\max _{0 \leq t \leq \frac{1}{\log \log A}}\|(D \Phi)(t, \cdot)\|_{\infty}>\log \log \log A .
$$

Proof of Lemma 6.3. We shall argue by contradiction. Assume (6.10) is not true, then

$$
\max _{0 \leq t \leq \frac{1}{\log \log A}}\|(D \Phi)(t, \cdot)\|_{\infty} \leq \log \log \log A .
$$

By the definition of the characteristic line $\Phi$, we have $W(t, x)=W_{0}(\tilde{\Phi}(t, x))$ where $\tilde{\Phi}$ is the inverse map of $\Phi$. By (6.11) and using a computation similar to (4.11), we get

$$
\begin{aligned}
\max _{0 \leq t \leq \frac{1}{\log \log A}}\|D W(t, \cdot)\|_{2} & \lesssim\left\|D W_{0}\right\|_{2} \cdot \max _{0 \leq t \leq \frac{1}{\log \log A}}\|D \Phi(t, \cdot)\|_{\infty} \\
& \lesssim \frac{1}{\log \log \log \log A} \cdot \log \log \log A \\
& \lesssim \log \log \log A .
\end{aligned}
$$

We shall need this estimate later.

The main idea is to compare $W$ with the other solution $\omega$ which solves the "unperturbed" equation

$$
\left\{\begin{array}{l}
\partial_{t} \omega+\Delta^{-1} \nabla^{\perp} \omega \cdot \nabla \omega+b(t, x) \cdot \nabla \omega=0, \\
\left.\omega\right|_{t=0}=g_{A} .
\end{array}\right.
$$

The perturbation theory requires a bit of work so we shall proceed in several steps.

Step 1. Set $\eta=W-\omega$. We first show that

$$
\|\eta(t, \cdot)\|_{B_{\infty, 1}^{0}} \lesssim 2^{-\frac{2}{3} A+}, \quad \forall 0 \leq t \leq \frac{1}{\log \log A} .
$$

Here and below we use the notation $X+$ as in (2.1). Also to simplify notations we shall write $\lesssim_{B_{1}}$ as $\lesssim$ (i.e. we suppress the notational dependence on $B_{1}$ ) since $A$ will be taken sufficiently large.

The equation for $\eta$ takes the form

$$
\left\{\begin{array}{l}
\partial_{t} \eta+\Delta^{-1} \nabla^{\perp} \eta \cdot \nabla W+\Delta^{-1} \nabla^{\perp} \omega \cdot \nabla \eta+b \cdot \nabla \eta+r \cdot \nabla W=0, \\
\eta(0)=0 .
\end{array}\right.
$$

By Lemma 6.1 and (6.8), we have

$$
\begin{aligned}
& \|r(t, \cdot)\|_{L^{\infty}(\operatorname{supp}(W(t, \cdot)))} \lesssim 4^{-A}, \\
& \|(D r)(t, \cdot)\|_{L^{\infty}(\operatorname{supp}(W(t, \cdot)))} \lesssim 2^{-A}, \\
& \left\|\left(D^{2} r\right)(t, \cdot)\right\|_{L^{\infty}(\operatorname{supp}(W(t, \cdot)))} \lesssim 1, \quad \forall 0 \leq t \leq \frac{1}{\log \log A} .
\end{aligned}
$$


Let $1<p<2$. By Sobolev embedding and (6.14), we compute

$$
\begin{aligned}
\frac{d}{d t}\left(\|\eta\|_{p}^{p}\right) & \lesssim\left\|\Delta^{-1} \nabla^{\perp} \eta\right\|_{\left(\frac{1}{p}-\frac{1}{2}\right)^{-1}}\|\nabla W\|_{2} \cdot\|\eta\|_{p}^{p-1}+4^{-A}\|\nabla W\|_{2} \cdot\|\eta\|_{p}^{p-1} \\
& \lesssim\|\nabla W\|_{2} \cdot\|\eta\|_{p}^{p}+4^{-A}\|\nabla W\|_{2} \cdot\|\eta\|_{p}^{p-1} .
\end{aligned}
$$

Therefore for $0 \leq t \leq \frac{1}{\log \log A}$, by using (6.12), we get

$$
\begin{aligned}
\|\eta(t, \cdot)\|_{p} & \lesssim 4^{-A} \int_{0}^{t} e^{(t-s) \log \log \log A} d s \cdot \log \log \log A \\
& \lesssim 4^{-A} \cdot \frac{\log \log \log A}{\log \log A} \lesssim 4^{-A} .
\end{aligned}
$$

This estimate is particularly good for $p=2-$.

On the other hand, for any $2 \leq q<\infty$, a standard energy estimate gives for any $0 \leq t \leq 1$,

$$
\begin{aligned}
\|\eta(t, \cdot)\|_{W^{2, q}} & \lesssim\|W(t, \cdot)\|_{W^{2, q}}+\|\omega(t, \cdot)\|_{W^{2, q}} \\
& \lesssim\left\|g_{A}\right\|_{W^{2, q}} \\
& \lesssim \frac{1}{\sqrt{\log A}} 2^{2(A+\log A)\left(1-\frac{1}{q}\right)} \\
& \lesssim 4^{A} .
\end{aligned}
$$

Interpolating the above with (6.15) then yields (6.13) (note that $\|\eta\|_{B_{\infty, 1}^{0}\left(\mathbb{R}^{2}\right)} \lesssim$ $\|\eta\|_{L^{2}\left(\mathbb{R}^{2}\right)}^{\frac{2}{3}}\|\Delta \eta\|_{L^{\infty}\left(\mathbb{R}^{2}\right)}^{\frac{1}{3}}$.)

Step 2. Let $\phi$ be the characteristic line associated with the equation for $\omega$, i.e.

$$
\left\{\begin{array}{l}
\partial_{t} \phi(t, x)=\left(\Delta^{-1} \nabla^{\perp} \omega+b\right)(t, \phi(t, x)) \\
\phi(t=0, x)=x, \quad x \in \mathbb{R}^{2}
\end{array}\right.
$$

We show that

$$
\max _{0 \leq t \leq \frac{1}{\log \log A}}\|\phi(t, \cdot)-\Phi(t, \cdot)\|_{\infty} \lesssim 2^{-\frac{4}{3} A+} .
$$

Set $Y(t, x)=\Phi(t, x)-\phi(t, x)$. By Lemma 6.1, we only need to consider $|x| \lesssim 2^{-A}$ sine $\phi(t, x)=\Phi(t, x)=x$ for $|x| \gg 2^{-A}$.

Then for $|x| \lesssim 2^{-A}$,

$$
\begin{aligned}
\frac{d}{d t} Y= & \left(\Delta^{-1} \nabla^{\perp} W\right)(\Phi)-\left(\Delta^{-1} \nabla^{\perp} \omega\right)(\phi) \\
& +b_{0}(t)\left(\begin{array}{c}
-Y_{1} \\
Y_{2}
\end{array}\right)+r(t, \Phi) \\
= & \left(\Delta^{-1} \nabla^{\perp} W\right)(\Phi)-\left(\Delta^{-1} \nabla^{\perp} W\right)(\phi)+\left(\Delta^{-1} \nabla^{\perp}(W-\omega)\right)(\phi) \\
& +b_{0}(t)\left(\begin{array}{c}
-Y_{1} \\
Y_{2}
\end{array}\right)+r(t, \Phi) .
\end{aligned}
$$

For $|x| \lesssim 2^{-A}$ and $0 \leq t \leq \frac{1}{\log \log A}$, we have $|\Phi(t, x)| \lesssim 2^{-A}$. By (6.8), we get

$$
\max _{|x| \lesssim 2^{-A}}|r(t, \Phi(t, x))| \lesssim 4^{-A}, \quad \forall 0 \leq t \leq \frac{1}{\log \log A} .
$$


Therefore

$\frac{d}{d t}(|Y(t, x)|) \lesssim\left(B_{0}+\|\mathcal{R} W\|_{\infty}\right) \cdot|Y(t, x)|+\left\|\Delta^{-1} \nabla^{\perp}(W-\omega)\right\|_{\infty}+4^{-A}$.

By using the usual log-interpolation inequality, we have

$$
\begin{aligned}
\|\mathcal{R} W\|_{\infty} & \lesssim\|W\|_{2}+\|W\|_{\infty} \log \left(10+\|W\|_{H^{2}}\right) \\
& \lesssim A
\end{aligned}
$$

On the other hand, by (6.13), we have

$$
\begin{aligned}
\left\|\Delta^{-1} \nabla^{\perp}(W-\omega)\right\|_{\infty} & \lesssim\|W-\omega\|_{1}^{\frac{1}{2}}\|W-\omega\|_{\infty}^{\frac{1}{2}} \\
& \lesssim 2^{-A} \cdot 2^{-\frac{1}{3} A+} \\
& \lesssim 2^{-\frac{4}{3} A+} .
\end{aligned}
$$

Plugging these estimates into (6.17) and integrating in time, we obtain

$$
\begin{aligned}
|Y(t, x)| & \lesssim \int_{0}^{t} e^{(t-s) C \cdot A}\left(4^{-A}+2^{-\frac{4}{3} A+}\right) d s \\
& \lesssim \int_{0}^{\frac{1}{\log \log A}} e^{\frac{C A}{\log \log A}}\left(4^{-A}+2^{-\frac{4}{3} A+}\right) d s \\
& \lesssim 2^{-\frac{4}{3} A+} .
\end{aligned}
$$

Step 3. Set $\tilde{J}(t)=(D \Phi)(t, x), J(t)=(D \phi)(t, x)$, then obviously

$$
\begin{aligned}
& \partial_{t} \tilde{J}=(\mathcal{R} W)(\Phi) \tilde{J}+b_{0}(t)\left(\begin{array}{cc}
-1 & 0 \\
0 & 1
\end{array}\right) \tilde{J}+(D r)(\Phi) \tilde{J}, \\
& \partial_{t} J=(\mathcal{R} \omega)(\phi) J+b_{0}(t)\left(\begin{array}{cc}
-1 & 0 \\
0 & 1
\end{array}\right) J .
\end{aligned}
$$

Let $q=\tilde{J}-J$. Then $q$ satisfies the equation

$$
\begin{aligned}
\partial_{t} q=( & (\mathcal{R} W)(\Phi)-(\mathcal{R} W)(\phi)) \tilde{J}+((\mathcal{R} W)(\phi)-(\mathcal{R} \omega)(\phi)) \tilde{J} \\
& +(\mathcal{R} \omega)(\phi) q+b_{0}(t)\left(\begin{array}{cc}
-1 & 0 \\
0 & 1
\end{array}\right) q+(D r)(\Phi) \tilde{J} .
\end{aligned}
$$

By (6.13), 6.16), 6.18), we obtain

$$
\begin{aligned}
\partial_{t}|q| \lesssim & \|D \mathcal{R} W\|_{\infty}|\Phi-\phi| \cdot\|\tilde{J}\|_{\infty}+2^{-\frac{2}{3} A+}\|\tilde{J}\|_{\infty} \\
& +A|q|+2^{-A}\|\tilde{J}\|_{\infty} \\
\lesssim & 2^{A+} 2^{-\frac{4}{3} A+} \log \log \log A+2^{-\frac{2}{3} A+} \log \log \log A+A|q| \\
& +2^{-A} \log \log \log A
\end{aligned}
$$

Integrating in time and noting $t \leq \frac{1}{\log \log A}$, we obtain

$$
\|q\|_{\infty} \lesssim 1
$$

But this obviously contradicts (6.7).

The next lemma is the main building block for our construction in the compactly supported data case.

Lemma 6.4. Suppose $f_{-1} \in C_{c}^{\infty}\left(\mathbb{R}^{2}\right)$ is a given real-valued function such that 
- for some $R_{0}>0$,

$$
\operatorname{supp}\left(f_{-1}\right) \subset\left\{x=\left(x_{1}, x_{2}\right): x_{1} \leq-2 R_{0}\right\} ;
$$

- $f_{-1}$ is an odd function of $x_{2}$, i.e.

$$
f_{-1}\left(x_{1}, x_{2}\right)=-f_{-1}\left(x_{1},-x_{2}\right), \quad \forall x \in \mathbb{R}^{2} .
$$

Then for any $0<\epsilon<\frac{R_{0}}{100}$, one can find $\delta_{0}=\delta_{0}\left(f_{-1}, \epsilon, R_{0}\right)>0,0<t_{0}=$ $t_{0}\left(f_{-1}, \epsilon, R_{0}\right)<\epsilon$, and $f_{0} \in C_{c}^{\infty}(B(0, \epsilon))$ ( $f_{0}$ depends only on $\left.\left(f_{-1}, \epsilon, R_{0}\right)\right)$ with the properties:

- $f_{0}$ is an odd function of $x_{2}$;

-

$$
\left\|f_{0}\right\|_{L^{1}}+\left\|f_{0}\right\|_{L^{\infty}}+\left\|f_{0}\right\|_{H^{1}}+\left\|f_{0}\right\|_{\dot{H}^{-1}} \leq \epsilon,
$$

such that for any $f_{1} \in C_{c}^{\infty}\left(\mathbb{R}^{2}\right)$ with

- $\operatorname{supp}\left(f_{1}\right) \subset\left\{x=\left(x_{1}, x_{2}\right): \quad x_{1} \geq R_{0}\right\}$;

- $\left\|f_{1}\right\|_{L^{1}}+\left\|f_{1}\right\|_{L^{\infty}} \leq \delta_{0}$

the following hold true:

Consider the Euler equation

$$
\left\{\begin{array}{l}
\partial_{t} \omega+\Delta^{-1} \nabla^{\perp} \omega \cdot \nabla \omega=0, \\
\left.\omega\right|_{t=0}=f_{-1}+f_{0}+f_{1},
\end{array}\right.
$$

then the smooth solution $\omega=\omega(t, x)$ satisfies the following properties:

(1) for any $0 \leq t \leq t_{0}$, we have the decomposition

$$
\omega(t, x)=\omega_{-1}(t, x)+\omega_{0}(t, x)+\omega_{1}(t, x),
$$

where

$$
\begin{aligned}
& \operatorname{supp}\left(\omega_{-1}(t)\right) \subset B\left(\operatorname{supp}\left(f_{-1}\right), \frac{1}{8} R_{0}\right) ; \\
& \operatorname{supp}\left(\omega_{0}(t)\right) \subset B\left(0, \epsilon+\frac{1}{8} R_{0}\right) ; \\
& \operatorname{supp}\left(\omega_{1}(t)\right) \subset B\left(\operatorname{supp}\left(f_{1}, \frac{1}{8} R_{0}\right)\right) .
\end{aligned}
$$

(2) $\left\|\omega_{0}(t=0, \cdot)\right\|_{H^{1}}=\left\|f_{0}\right\|_{H^{1}} \leq \epsilon$, but

$$
\left\|\omega_{0}\left(t_{0}, \cdot\right)\right\|_{\dot{H}^{1}}>\frac{1}{\epsilon} .
$$

Proof of Lemma 6.4. The decomposition (6.20) is a simple consequence of finite transportation speed. Therefore we only need to show how to choose $f_{0}$ to achieve (6.21) and the other conditions.

Consider first the equation

$$
\left\{\begin{array}{l}
\partial_{t} \omega^{(1)}+\Delta^{-1} \nabla^{\perp} \omega^{(1)} \cdot \nabla \omega^{(1)}=0, \\
\left.\omega^{(1)}\right|_{t=0}=f_{-1}+g_{A},
\end{array}\right.
$$

where $g_{A}$ was defined in (6.4) and we shall choose $A$ sufficiently large.

For $0 \leq t \leq \frac{1}{\log \log A}$, we decompose the solution $\omega^{(1)}(t)$ to (6.22) as

$$
\omega^{(1)}(t, x)=\omega_{-1}^{(1)}(t, x)+\omega_{0}^{(1)}(t, x),
$$


with

$$
\begin{aligned}
& \operatorname{supp}\left(\omega_{-1}^{(1)}(t, \cdot)\right) \subset B\left(\operatorname{supp}\left(f_{-1}\right), \frac{1}{10} R_{0}\right), \\
& \left.\operatorname{supp}\left(\omega_{0}^{(1)}\right)(t, \cdot)\right) \subset B\left(\operatorname{supp}\left(g_{A}\right), \frac{1}{10} R_{0}\right) .
\end{aligned}
$$

Obviously $\omega_{0}^{(1)}(t)$ satisfies the equation

$$
\left\{\begin{array}{l}
\partial_{t} \omega_{0}^{(1)}+\Delta^{-1} \nabla^{\perp} \omega_{0}^{(1)} \cdot \nabla \omega_{0}^{(1)}+\Delta^{-1} \nabla^{\perp} \omega_{-1}^{(1)}(t) \cdot \nabla \omega_{0}^{(1)}=0, \\
\left.\omega_{0}^{(1)}\right|_{t=0}=g_{A} .
\end{array}\right.
$$

Since by assumption $f_{-1}$ is odd in $x_{2}$ and $g_{A}$ is also odd in $x_{2}$, it is easy to check that both $\omega_{-1}^{(1)}(t)$ and $\omega_{0}^{(1)}(t)$ are odd functions of $x_{2}$. Therefore we have

$$
\begin{aligned}
& \left(\Delta^{-1} \partial_{22} \omega_{-1}^{(1)}\right)\left(t, x_{1}, 0\right)=0, \\
& \left(\Delta^{-1} \partial_{11} \omega_{-1}^{(1)}\right)\left(t, x_{1}, 0\right)=0, \\
& \left(\Delta^{-1} \partial_{1} \omega_{-1}^{(1)}\right)\left(t, x_{1}, 0\right)=0, \quad \forall 0 \leq t \leq \frac{1}{\log \log A}, x_{1} \in \mathbb{R} .
\end{aligned}
$$

Now let $\xi(t)$ solve the ODE

$$
\left\{\begin{array}{l}
\frac{d}{d t} \xi(t)=\left(\Delta^{-1} \partial_{2} \omega_{-1}^{(1)}\right)(t, \xi(t), 0) \\
\xi(0)=0
\end{array}\right.
$$

Since for $0 \leq t \leq \frac{1}{\log \log A}$ and $A$ is sufficiently large, the function $\omega_{-1}^{(1)}$ is supported away from the origin (see (6.23)), it is easy to check that the function $\left(\Delta^{-1} \partial_{2} \omega_{-1}^{(1)}\right)(t, \cdot)$ is smooth and has uniform (independent of $A$ ) Sobolev bounds in a small neighborhood of the origin. Thus $\xi(t)$ is well-defined and remains close to the origin for $t \leq \frac{1}{\log \log A}$.

By (6.25), we have

$$
\begin{aligned}
\left(-\Delta^{-1} \partial_{2} \omega_{-1}^{(1)}\right)\left(t, \xi(t)+y_{1}, y_{2}\right)=- & \left(\Delta^{-1} \partial_{2} \omega_{-1}^{(1)}\right)(t, \xi(t), 0)-\left(\Delta^{-1} \partial_{12} \omega_{-1}^{(1)}\right)(t, \xi(t), 0) y_{1} \\
& +r_{1}^{(1)}(t, y) \\
\left(\Delta^{-1} \partial_{1} \omega_{-1}^{(1)}\right)\left(t, \xi(t)+y_{1}, y_{2}\right)= & \left(\Delta^{-1} \partial_{12} \omega_{-1}^{(1)}\right)(t, \xi(t), 0) y_{2}+r_{2}^{(1)}(t, y)
\end{aligned}
$$

where for $0 \leq t \leq \frac{1}{\log \log A}$,

$$
\begin{aligned}
& \left|r_{1}^{(1)}(t, y)\right|+\left|r_{2}^{(1)}(t, y)\right| \lesssim_{f_{-1}, R_{0}}|y|^{2}, \\
& \left|\left(D r_{1}^{(1)}\right)(t, y)\right|+\left|\left(D r_{2}^{(1)}\right)(t, y)\right| \lesssim_{f_{-1}, R_{0}}|y|, \\
& \left|\left(D^{2} r_{1}^{(1)}\right)(t, y)\right|+\left|\left(D^{2} r_{2}^{(1)}\right)(t, y)\right| \lesssim_{f_{-1}, R_{0}} 1 .
\end{aligned}
$$

By (6.26), we may write the above more compactly as

$$
\begin{aligned}
& \left(\Delta^{-1} \nabla^{\perp} \omega_{-1}^{(1)}\right)\left(t, \xi(t)+y_{1}, y_{2}\right) \\
= & \left(\begin{array}{c}
-\frac{d}{d t} \xi(t) \\
0
\end{array}\right)+b_{0}(t)\left(\begin{array}{c}
-y_{1} \\
y_{2}
\end{array}\right)+r(t, y),
\end{aligned}
$$


where

$$
\begin{aligned}
& \left|b_{0}(t)\right| \lesssim_{f_{-1}, R_{0}} 1, \\
& |r(t, y)| \lesssim_{-1}, R_{0}|y|^{2}, \\
& |(D r)(t, y)| \lesssim_{f_{-1}, R_{0}}|y|, \\
& \left|\left(D^{2} r\right)(t, y)\right| \lesssim_{f_{-1}, R_{0}} 1 .
\end{aligned}
$$

Now we make a change of variable and set

$$
\begin{aligned}
& x=\left(\xi(t)+y_{1}, y_{2}\right), \\
& \omega_{0}^{(1)}(t, x)=\omega_{0}^{(1)}\left(t, \xi(t)+y_{1}, y_{2}\right)=: W_{0}^{(1)}\left(t, y_{1}, y_{2}\right) .
\end{aligned}
$$

By using (6.27) and the above expressions, we can write (6.24) as

$$
\begin{aligned}
& \partial_{t} W_{0}^{(1)}(t, y)+\left(\Delta^{-1} \nabla^{\perp} W_{0}^{(1)} \cdot \nabla W_{0}^{(1)}\right)(t, y) \\
& \quad+b_{0}(t)\left(\begin{array}{c}
-y_{1} \\
y_{2}
\end{array}\right) \cdot \nabla W_{0}^{(1)}(t, y)+r(t, y) \cdot \nabla W_{0}^{(1)}(t, y)=0,
\end{aligned}
$$

where $b_{0}(t), r(t, y)$ satisfies (6.28).

By Lemma 6.1, we have for $0 \leq t \leq \frac{1}{\log \log A}$,

$$
\operatorname{supp}\left(W_{0}^{(1)}(t, \cdot)\right) \subset B(0, \tilde{R}), \quad \text { with } \tilde{R} \sim 2^{-A} .
$$

Therefore by (6.28) and Lemma 6.3. we have for $A$ sufficiently large,

$$
\max _{0 \leq t \leq \frac{1}{\log \log A}}\|(D \Phi)(t, \cdot)\|_{\infty}>\log \log \log A,
$$

were $\Phi$ is the forward characteristic line associated with $W_{0}^{(1)}$ (see (6.9)).

Let $\phi$ be the characteristic line solving the ODE

$$
\left\{\begin{array}{l}
\partial_{t} \phi(t, x)=\left(\Delta^{-1} \nabla^{\perp} \omega_{0}^{(1)}+\Delta^{-1} \nabla^{\perp} \omega_{-1}^{(1)}\right)(t, \phi(t, x)), \\
\phi(t=0, x)=x .
\end{array}\right.
$$

Denote by $\tilde{\Phi}, \tilde{\phi}$ the inverse maps of $\Phi$ and $\phi$ respectively. By (6.29), it is easy to check that

$$
\tilde{\Phi}(t, y)=\tilde{\phi}(t, \xi(t)+y), \quad \text { for any } t \geq 0 \text { and } y \in \mathbb{R}^{2} .
$$

Therefore by (6.30),

$$
\max _{0 \leq t \leq \frac{1}{\log \log A}}\|(D \phi)(t, \cdot)\|_{\infty}>\log \log \log A .
$$

Now we just need to modify slightly the proof of Proposition 4.2. Note that one can always choose the perturbation $\beta(x)$ (see (4.14) ) to be odd in $x_{1}$ and $x_{2}$, for example,

$$
\beta(x)=\frac{1}{k} \sin \left(k x_{1}\right) \sin \left(x_{2}\right) b(x) \frac{1}{\sqrt{M}} .
$$

Denote by $\tilde{g}_{A}$ the perturbed initial data and let $\tilde{\omega}^{(1)}$ be the solution to

$$
\left\{\begin{array}{l}
\partial_{t} \tilde{\omega}^{(1)}+\Delta^{-1} \nabla^{\perp} \tilde{\omega}^{(1)} \cdot \nabla \tilde{\omega}^{(1)}=0, \\
\left.\tilde{\omega}^{(1)}\right|_{t=0}=f_{-1}+\tilde{g}_{A} .
\end{array}\right.
$$


Similar to $\omega^{(1)}($ see $(\underline{6.22})$ ), we also have the decomposition similar to that in (6.23):

$$
\tilde{\omega}^{(1)}=\tilde{\omega}_{-1}^{(1)}+\tilde{\omega}_{0}^{(1)}
$$

By our choice of perturbation (and taking $A$ sufficiently large), we have

$$
\max _{0 \leq t \leq \frac{1}{\log \log A}}\left\|\tilde{\omega}_{0}^{(1)}(t, \cdot)\right\|_{\dot{H}^{1}}>(\log \log \log A)^{\frac{1}{3}} .
$$

Let $f_{0}=\tilde{g}_{A}$. We then compare $\tilde{\omega}^{(1)}$ with $\omega$ which solves

$$
\left\{\begin{array}{l}
\partial_{t} \omega+\Delta^{-1} \nabla^{\perp} \omega \cdot \nabla \omega=0, \\
\left.\omega\right|_{t=0}=f_{-1}+f_{0}+f_{1},
\end{array}\right.
$$

with $f_{1} \in C_{c}^{\infty}\left(\mathbb{R}^{2}\right)$ satisfying

- $\operatorname{supp}\left(f_{1}\right) \subset\left\{x=\left(x_{1}, x_{2}\right): \quad x_{1} \geq \frac{1}{2} R_{0}\right\} ;$

- $\left\|f_{1}\right\|_{L^{1}}+\left\|f_{1}\right\|_{L^{\infty}} \leq \delta_{0}$

and $\delta_{0}$ is to be taken sufficiently small.

By an argument similar to the proof of (5.18), we then have (see (6.20) for the definition of $\left.\omega_{0}(t)\right)$

$$
\max _{0 \leq t \leq \frac{1}{\log \log A}}\left\|\omega_{0}(t)-\tilde{\omega}_{0}^{(1)}(t)\right\|_{H^{2}} \lesssim_{\epsilon, f_{-1}, R_{0}} \delta_{0} .
$$

Therefore (6.21) follows by choosing $\delta_{0}$ sufficiently small.

We are now state a weaker version of Theorem 1.6 whose proof is simpler (but elucidates the main ideas). The main difference is that the constructed solution $\omega$ is only in $L^{\infty}$ rather than being continuous. This already gives the desired illposedness in $H^{1}$ for compactly supported initial data. We defer the proof of Theorem 1.6 slightly later since it needs an additional perturbation argument in $C^{0}$.

Theorem 6.5. Let $\omega_{0}^{(g)} \in C_{c}^{\infty}\left(\mathbb{R}^{2}\right) \cap \dot{H}^{-1}\left(\mathbb{R}^{2}\right)$ be any given vorticity function which is odd in $x_{2}$. For any such $\omega_{0}^{(g)}$ and any $\epsilon>0$, we can find a perturbation $\omega_{0}^{(p)}: \mathbb{R}^{2} \rightarrow \mathbb{R}$ such that the following hold true:

(1) $\omega_{0}^{(p)}$ is compactly supported (in a ball of radius $\leq 1$ ), continuous and

$$
\left\|\omega_{0}^{(p)}\right\|_{\dot{H}^{1}\left(\mathbb{R}^{2}\right)}+\left\|\omega_{0}^{(p)}\right\|_{L^{\infty}\left(\mathbb{R}^{2}\right)}+\left\|\omega_{0}^{(p)}\right\|_{\dot{H}^{-1}\left(\mathbb{R}^{2}\right)}<\epsilon .
$$

(2) Let $\omega_{0}=\omega_{0}^{(g)}+\omega_{0}^{(p)}$. Corresponding to $\omega_{0}$ there exists a unique time-global solution $\omega=\omega(t)$ to the Euler equation satisfying

$$
\sup _{0<t<\infty}\left(\|\omega(t, \cdot)\|_{\infty}+\|\omega(t, \cdot)\|_{\dot{H}^{-1}}\right)<\infty .
$$

(3) $\omega(t)$ has additional local regularity in the following sense: there exists $x_{*} \in$ $\mathbb{R}^{2}$ such that for any $x \neq x_{*}$, there exists a neighborhood $N_{x} \ni x, t_{x}>0$ such that $w(t, \cdot) \in C^{\infty}\left(N_{x}\right)$ for any $0 \leq t \leq t_{x}$.

(4) For any $0<t_{0} \leq 1$, we have

$$
\operatorname{ess}^{-\sup _{0<t \leq t_{0}}}\|\omega(t, \cdot)\|_{\dot{H}^{1}}=+\infty .
$$


More precisely, there exist $0<t_{n}^{1}<t_{n}^{2}<\frac{1}{n}$, open precompact sets $\Omega_{n}$, $n=1,2,3, \cdots$ such that $\omega(t) \in C^{\infty}\left(\Omega_{n}\right)$ for all $0 \leq t \leq t_{n}^{2}$, and

$$
\|\nabla \omega(t, \cdot)\|_{L^{2}\left(\Omega_{n}\right)}>n, \quad \forall t \in\left[t_{n}^{1}, t_{n}^{2}\right] .
$$

6.1. Proof of Theorem 6.5. We begin by noting that the support condition in Statement (1) ("compactly supported in a ball of radius $\leq 1$ ") is rather easy to achieve: one only needs to change the parameters of the distances between the patch solutions in our construction below. Similar comment also applies to the condition " $\left\|\omega_{0}^{(p)}\right\|_{\dot{H}^{1}\left(\mathbb{R}^{2}\right)}+\left\|\omega_{0}^{(p)}\right\|_{L^{\infty}\left(\mathbb{R}^{2}\right)}+\left\|\omega_{0}^{(p)}\right\|_{\dot{H}^{-1}\left(\mathbb{R}^{2}\right)}<\epsilon "$. Therefore we shall ignore all these conditions below. In particular to simplify notation we will construct $\omega_{0}^{(p)}$ of order 1. Also without loss of generality we may assume $\omega_{0}^{(g)}$ is supported (say) in a ball of radius $\leq \frac{1}{1000}$.

Define $z_{0}=(-2,0), z_{1}=(0,0)$. For each integer $j \geq 2$, define

$$
z_{j}=\left(z_{j}^{(1)}, 0\right)=\left(\sum_{k=1}^{j-1} \frac{100}{2^{k}}, 0\right)
$$

Obviously for any $j \geq 2$, we have

$$
\begin{aligned}
& \left|z_{j}-z_{j-1}\right|=\frac{100}{2^{j-1}}, \\
& \left|z_{j+1}-z_{j}\right|=\frac{100}{2^{j}} .
\end{aligned}
$$

We shall choose $z_{j}, j \geq 0$ to be the center of the $j^{\text {th }}$ patch.

Now define $h_{0}(x)=\omega_{0}^{(g)}\left(x-z_{0}\right)$ and $\delta_{0}=1$. By Lemma 6.4 with $f_{-1}=h_{0}$, $R_{0}=\frac{1}{4}, \epsilon=1 / 800$, we can find $\delta_{1}>0,0<t_{1}<\frac{1}{2}$, and $h_{1} \in C_{c}^{\infty}\left(B\left(0, \frac{1}{800}\right)\right)$ with the properties

- $h_{1}$ is an odd function of $x_{2}$

- $\left\|h_{1}\right\|_{L^{1}}+\left\|h_{1}\right\|_{L^{\infty}}+\left\|h_{1}\right\|_{H^{1}}+\left\|h_{1}\right\|_{\dot{H}^{-1}} \leq \frac{1}{8}$

such that for any $\tilde{f} \in C_{c}^{\infty}\left(\mathbb{R}^{2}\right)$ with

- $\operatorname{supp}(\tilde{f}) \subset\left\{x=\left(x_{1}, x_{2}\right): \quad x_{1} \geq \frac{1}{4}\right\}$

- $\|\tilde{f}\|_{L^{1}}+\|\tilde{f}\|_{L^{\infty}} \leq \delta_{1}$,

the following hold true:

For the Euler equation

$$
\left\{\begin{array}{l}
\partial_{t} \omega+\Delta^{-1} \nabla^{\perp} \omega \cdot \nabla \omega=0, \\
\left.\omega\right|_{t=0}=h_{0}+h_{1}+\tilde{f},
\end{array}\right.
$$

the smooth solution $\omega=\omega(t)$ satisfies:

(1) For any $0 \leq t \leq t_{1}, \omega(t)$ can be decomposed as

$$
\omega(t, x)=\omega_{h_{0}}(t, x)+\omega_{h_{1}}(t, x)+\omega_{\tilde{f}}(t, x),
$$

where

$$
\begin{aligned}
& \operatorname{supp}\left(\omega_{h_{0}}(t, \cdot)\right) \subset B\left(0,-2+\frac{1}{32}\right), \\
& \operatorname{supp}\left(\omega_{h_{1}}(t, \cdot)\right) \subset B\left(0, \frac{1}{8}+\frac{1}{32}\right), \\
& \operatorname{supp}\left(\omega_{\tilde{f}}(t, \cdot)\right) \subset\left\{x=\left(x_{1}, x_{2}\right): \quad x_{1} \geq \frac{1}{4}+\frac{1}{32}\right\} ;
\end{aligned}
$$


$(2)$

$$
\left\|\omega_{h_{1}}\left(t_{1}, \cdot\right)\right\|_{\dot{H}^{1}}>8
$$

We now inductively assume that for $1 \leq i \leq j$, we have chosen $h_{i} \in C_{c}^{\infty}\left(B\left(z_{i}, \frac{1}{2^{i+9}}\right)\right)$ which is odd in $x_{2}, 0<t_{i}<\frac{1}{2^{i}}, \delta_{i}>0$, with

$$
\begin{aligned}
& \left\|h_{i}\right\|_{L^{1}}+\left\|h_{i}\right\|_{L^{\infty}}+\left\|h_{i}\right\|_{H^{1}}+\left\|h_{i}\right\|_{\dot{H}^{-1}} \\
& \quad \leq \frac{1}{2^{i}} \min _{0 \leq k<i} \delta_{k},
\end{aligned}
$$

such that for any $\tilde{f} \in C_{c}^{\infty}\left(\mathbb{R}^{2}\right)$ with

- $\operatorname{supp}(\tilde{f}) \subset\left\{x=\left(x_{1}, x_{2}\right): \quad x_{1} \geq z_{i+1}^{(1)}-\frac{1}{2^{2}}\right\}$ (see (6.33) $)$ for the definition of $z_{j}^{(1)}$;

- $\|\tilde{f}\|_{L^{1}}+\|\tilde{f}\|_{L^{\infty}} \leq \delta_{i}$

the solution $\omega(t)$ to the equation

$$
\left\{\begin{array}{l}
\partial_{t} \omega+\Delta^{-1} \nabla^{\perp} \omega \cdot \nabla \omega=0 \\
\left.\omega\right|_{t=0}=\sum_{l=1}^{i-1} h_{l}+h_{i}+\tilde{f},
\end{array}\right.
$$

satisfies the properties:

(1) for any $0 \leq t \leq t_{i}$, we have the decomposition

$$
\omega(t, x)=\omega_{\leq i-1}(t, x)+\omega_{i}(t, x)+\omega_{\tilde{f}}(t, x),
$$

where

$$
\begin{array}{ll}
\operatorname{supp}\left(\omega_{\leq i-1}(t, \cdot)\right) \subset\left\{x=\left(x_{1}, x_{2}\right):\right. & \left.x_{1} \leq z_{i-1}^{(1)}+\frac{1}{2^{i}}\right\} ; \\
\operatorname{supp}\left(\omega_{i}(t, \cdot)\right) \subset\left\{x=\left(x_{1}, x_{2}\right):\right. & \left.\left|x-z_{i}\right| \leq \frac{1}{2^{i}}\right\} ; \\
\operatorname{supp}\left(\omega_{\tilde{f}}(t, \cdot)\right) \subset\left\{x=\left(x_{1}, x_{2}\right):\right. & \left.x_{1} \geq z_{i+1}^{(1)}-\frac{1}{2^{i}}\right\} ;
\end{array}
$$

(2) $\left\|\omega_{i}\left(t_{i}, \cdot\right)\right\|_{\dot{H}^{1}}>2^{i}$.

Then for $i=j+1$, by shifting the coordinate axis to $z_{j+1}$ if necessary, we can apply Lemma 6.4 with $f_{-1}=\sum_{i=0}^{j} h_{i}, \epsilon \ll \frac{1}{2^{i+1}} \min _{0 \leq k \leq i} \delta_{k}$, and choose $h_{j+1} \in C_{c}^{\infty}\left(B\left(z_{j+1}, \frac{1}{2^{j+9}}\right)\right.$ to satisfy all the needed properties similar to the $i^{\text {th }}$ step. This way we have completely specified the profiles of all $h_{j}, j=0,1,2, \cdots$.

Now we define the initial data

$$
\omega_{0}=\sum_{j=0}^{\infty} h_{j} .
$$

It is easy to check that $\omega_{0}$ is compactly supported and $\omega_{0} \in L^{\infty} \cap \dot{H}^{-1} \cap H^{1}$. We can then appeal to Yudovich theory to construct the corresponding solution in $L^{\infty}$. However here for the sake of completeness we shall carry out a direct construction which also yields additional local regularity of the solution. For simplicity we shall be content with constructing a local solution on some time interval $\left[0, T_{0}\right]$ with $T_{0}>0$. The breakdown of $\dot{H}^{1}$ regularity will happen arbitrarily close to time $t=0$. 
To this end, denote the approximating initial data

$$
\omega_{0}^{(J)}=\sum_{j=0}^{J} h_{j}
$$

and let $\omega^{(J)}$ be the solution to the Euler equation

$$
\left\{\begin{array}{l}
\partial_{t} \omega^{(J)}+\Delta^{-1} \nabla^{\perp} \omega^{(J)} \cdot \nabla \omega^{(J)}=0 \\
\left.\omega^{(J)}\right|_{t=0}=\omega_{0}^{(J)}
\end{array}\right.
$$

By using $L^{p}, 1 \leq p \leq \infty$ conservation of vorticity, $L^{2}$ conservation of velocity, it is easy to check that

$$
\sup _{J} \sup _{0 \leq t<\infty}\left(\left\|\omega^{(J)}(t, \cdot)\right\|_{L^{1}}+\left\|\omega^{(J)}(t, \cdot)\right\|_{L^{\infty}}+\left\|\omega^{(J)}(t, \cdot)\right\|_{\dot{H}^{-1}}\right) \lesssim 1 .
$$

We now verify that $\left(\omega^{(J)}(t)\right)$ forms a Cauchy sequence in the Banach space $C_{t}^{0} \dot{H}^{-1}\left(\left[0, T_{0}\right]\right)$ for some $T_{0}>0$ sufficiently small.

Denote $\eta^{(J+1)}=\omega^{(J+1)}-\omega^{(J)}$. Then

$$
\left\{\begin{array}{l}
\partial_{t} \eta^{(J+1)}+\Delta^{-1} \nabla^{\perp} \eta^{(J+1)} \cdot \nabla \omega^{(J+1)}+\Delta^{-1} \nabla^{\perp} \omega^{(J)} \cdot \nabla \eta^{(J+1)}=0, \\
\left.\eta^{(J+1)}\right|_{t=0}=h_{J+1} .
\end{array}\right.
$$

Multiplying both sides of (6.38) by $(-\Delta)^{-1} \eta^{(J+1)}$, integrating by parts and using (6.37), we have

$$
\begin{aligned}
& \frac{d}{d t}\left(\left\||\nabla|^{-1} \eta^{(J+1)}\right\|_{2}^{2}\right) \\
\leq & \left\||\nabla|^{-1} \eta^{(J+1)}\right\|_{2}^{2} \cdot\left\|\omega^{(J+1)}\right\|_{\infty}+\left|\int_{\mathbb{R}^{2}}\left(\Delta^{-1} \nabla^{\perp} \omega^{(J)} \cdot \nabla \eta^{(J+1)}\right) \Delta^{-1} \eta^{(J+1)} d x\right| \\
& \lesssim\left\||\nabla|^{-1} \eta^{(J+1)}\right\|_{2}^{2}+\left|\int_{\mathbb{R}^{2}}\left(\Delta^{-1} \nabla^{\perp} \omega^{(J)} \cdot \nabla \eta^{(J+1)}\right) \Delta^{-1} \eta^{(J+1)} d x\right| .
\end{aligned}
$$

Here note that since $\eta^{(J+1)}$ is an odd function of $x_{2}$, we have $\widehat{\eta^{(J+1)}}(\xi=0)=0$. From this it is easy to check that $\Delta^{-1} \eta^{(J+1)}$ is a smooth function. By successive integration by parts and Hölder, we have

$$
\begin{aligned}
&\left|\int_{\mathbb{R}^{2}}\left(\Delta^{-1} \nabla^{\perp} \omega^{(J)} \cdot \nabla \eta^{(J+1)}\right) \Delta^{-1} \eta^{(J+1)} d x\right| \\
&=\left|\int_{\mathbb{R}^{2}}\left(\Delta^{-1} \nabla^{\perp} \omega^{(J)} \cdot \nabla\left(\Delta \Delta^{-1} \eta^{(J+1)}\right)\right) \Delta^{-1} \eta^{(J+1)} d x\right| \\
&=\left|\sum_{k=1}^{2} \int_{\mathbb{R}^{2}}\left(\Delta^{-1} \nabla^{\perp} \partial_{k} \omega^{(J)} \cdot \nabla\left(\Delta^{-1} \eta^{(J+1)}\right)\right) \partial_{k} \Delta^{-1} \eta^{(J+1)} d x\right| \\
& \lesssim\left\|\mathcal{R}_{i j} \omega^{(J)}\right\|_{p} \cdot\left\||\nabla|^{-1} \eta^{(J+1)}\right\|_{\frac{2 p}{p-1}}^{2},
\end{aligned}
$$

where $\mathcal{R}_{i j}$ is the usual Riesz transform and $1<p<\infty$.

Now recall that for $2 \leq p<\infty$, we have

$$
\left\|\mathcal{R}_{i j} \omega^{(J)}\right\|_{p} \leq C_{1} \cdot p\left\|\omega^{(J)}\right\|_{p}
$$

where $C_{1}>0$ is an absolute constant. 
By Hölder and (6.37), we have

$$
\begin{aligned}
\left\||\nabla|^{-1} \eta^{(J+1)}\right\|_{\frac{2 p}{p-1}} & \leq\left\||\nabla|^{-1} \eta^{(J+1)}\right\|_{2}^{\frac{p-1}{p}} \cdot\left\||\nabla|^{-1} \eta^{(J+1)}\right\|_{\infty}^{\frac{1}{p}} \\
& \lesssim\left\||\nabla|^{-1} \eta^{(J+1)}\right\|_{2}^{\frac{p-1}{p}} .
\end{aligned}
$$

Plugging the last two estimates and (6.40) into (6.39), we obtain

$$
\frac{d}{d t}\left(\left\||\nabla|^{-1} \eta^{(J+1)}\right\|_{2}^{2}\right) \lesssim\left\||\nabla|^{-1} \eta^{(J+1)}\right\|_{2}^{2}+p \cdot\left\||\nabla|^{-1} \eta^{(J+1)}\right\|_{2}^{\frac{2(p-1)}{p}} .
$$

Now denote

$$
a(t):=\left\||\nabla|^{-1} \eta^{(J+1)}(t)\right\|_{2}^{2} .
$$

We then obtain for some absolute constant $C>0$,

$$
a^{\prime}(t) \leq C \cdot\left(a(t)+p a(t)^{\frac{p-1}{p}}\right) .
$$

By (6.37), we have $a(t)=a(t)^{\frac{p-1}{p}} a(t)^{\frac{1}{p}} \lesssim a(t)^{\frac{p-1}{p}}$. Hence

$$
a^{\prime}(t) \leq C^{\prime} p a(t)^{\frac{p-1}{p}}, \quad \forall t \geq 0,
$$

where $C^{\prime}>0$ is another absolute constant. Integrating in time gives the inequality,

$$
a(t)^{\frac{1}{p}} \leq a(0)^{\frac{1}{p}}+C^{\prime} \cdot t, \quad \forall t \geq 0 .
$$

We now choose $T_{0}>0$ such that $C^{\prime} T_{0} \leq \frac{1}{4}$. Then for all $0 \leq t \leq T_{0}$,

$$
a(t) \leq\left(a(0)^{\frac{1}{p}}+\frac{1}{4}\right)^{p} .
$$

Now since $a(0)=\left\||\nabla|^{-1} \eta^{(J+1)}(0)\right\|_{2}^{2}=\left\||\nabla|^{-1} h_{J+1}\right\|_{2}^{2} \leq 4^{-(J+1)}$ (see (6.34) $)$, taking $p=J$ gives us

$$
\begin{aligned}
a(t) & \leq\left(4^{-\frac{J+1}{p}}+\frac{1}{4}\right)^{p} \\
& \leq\left(\frac{1}{2}\right)^{p}=2^{-J}, \quad \forall 0 \leq t \leq T_{0} .
\end{aligned}
$$

Therefore

$$
\max _{0 \leq t \leq T_{0}}\left\||\nabla|^{-1} \eta^{(J+1)}(t, \cdot)\right\|_{2}^{2} \leq 2^{-J}
$$

and consequently $\left(\omega^{(J)}\right)$ is Cauchy in $C_{t}^{0} \dot{H}^{-1}\left(\left[0, T_{0}\right]\right)$. Taking the limit $J \rightarrow \infty$ gives us the desired solution in $L^{1} \cap L^{\infty} \cap \dot{H}^{-1}$. By a simple interpolation argument we also have $\left(\omega^{(J)}\right)$ is Cauchy in $L^{p}$ for any $1<p<\infty$.

Set $x_{*}=\lim _{j \rightarrow \infty} z_{j}=(100,0)$. We now prove statement (3) and (4) in Theorem 6.5. Fix any integer $n \geq 2$ and we choose $t_{n}<\frac{1}{2^{n}}$ in the same way as specified in (6.34). By our way of construction, the fact that $\left(\omega^{(J)}\right)$ is Cauchy in $L^{p}$ for any $1<p<\propto 5$ and (a version of) Lemma 5.1, we have that the limit solution $\omega$ obeys the following decomposition similar to that in 6.35 :

More precisely define $t_{n}^{2}=t_{n}$, then for any $0 \leq t \leq t_{n}^{2}$, we have

$$
\omega(t, x)=\omega_{<n}(t, x)+\omega_{n}(t, x)+\omega_{>n}(t, x),
$$

\footnotetext{
${ }^{5}$ This is used to show the contraction of all higher Sobolev norms in patches away from the limiting point $x_{*}$ (and in a very small time interval) which will yield the $C^{\infty}$ regularity of $\omega_{<n}$ and $\omega_{n}$ in 6.41) below.
} 
where $\omega_{<n}(t, \cdot) \in C_{c}^{\infty}\left(\Omega_{<n}\right), \omega_{n}(t, \cdot) \in C_{c}^{\infty}\left(\Omega_{n}\right)$, and

$$
\begin{aligned}
& \Omega_{<n}:=\left\{x=\left(x_{1}, x_{2}\right): \quad|x|<1000 \text { and } x_{1}<z_{n-1}^{(1)}+\frac{2}{2^{n}}\right\} ; \\
& \Omega_{n}:=\left\{x=\left(x_{1}, x_{2}\right): \quad\left|x-z_{n}\right|<\frac{2}{2^{n}}\right\} ; \\
& \operatorname{supp}\left(\omega_{>n}(t, \cdot)\right) \subset\left\{x=\left(x_{1}, x_{2}\right): \quad x_{1} \geq z_{n+1}^{(1)}-\frac{1}{2^{n}}\right\} ;
\end{aligned}
$$

Furthermore we can choose $t_{n}^{1}<t_{n}^{2}\left(t_{n}^{1}\right.$ is sufficiently close to $\left.t_{n}^{2}\right)$ such that

$$
\left\|\omega_{n}(t)\right\|_{\dot{H}^{1}}>n, \quad \forall t \in\left[t_{n}^{1}, t_{n}^{2}\right] .
$$

Therefore statement (4) in Theorem 6.5 is proved. Now for statement (3) we discuss two cases. If $x=\left(x_{1}, x_{2}\right) \neq x_{*}=(100,0)$ and $x_{1} \geq 100$, then by using finite transportation speed we can find a neighborhood $N_{x}$ of $x$ and $t_{x}>0$ sufficiently small such that $\omega(t, x)=0$ for any $0 \leq t \leq t_{x}$ and $x \in N_{x}$. Similarly we can treat the case $x=\left(x_{1}, x_{2}\right), x_{1}<100$ and $|x|>500$. On the other hand if $x=\left(x_{1}, x_{2}\right)$ and $x_{1}<100$ with $|x| \leq 500$, then we can find $n$ sufficiently large such that $x \in \Omega_{<n}$. Obviously we just need to define $N_{x}=\Omega_{<n}$ and $t_{x}=t_{n}^{2}$ so that $\omega(t, \cdot) \in C^{\infty}\left(N_{x}\right)$ for all $0 \leq t \leq t_{x}$.

This concludes the proof of Theorem 6.5

We now state the $C^{0}$-perturbation lemma needed for the proof of Theorem 1.6.

Lemma 6.6. Let $R_{0}>0$ and $f \in C_{c}^{\infty}\left(B\left(0, R_{0}\right)\right), g \in C_{c}^{\infty}\left(B\left(0, R_{0}\right)\right)$. Let $\omega^{a}$ and $\omega$ be smooth solutions to the following $2 D$ Euler equations:

$$
\begin{aligned}
& \left\{\begin{array}{l}
\partial_{t} \omega^{a}+\left(u^{a} \cdot \nabla\right) \omega^{a}=0, \quad 0<t \leq 1, x \in \mathbb{R}^{2}, \\
u^{a}=\Delta^{-1} \nabla^{\perp} \omega^{a}, \\
\left.\omega^{a}\right|_{t=0}=f .
\end{array}\right. \\
& \left\{\begin{array}{l}
\partial_{t} \omega+(u \cdot \nabla) \omega=0, \quad 0<t \leq 1, x \in \mathbb{R}^{2}, \\
u=\Delta^{-1} \nabla^{\perp} \omega, \\
\left.\omega\right|_{t=0}=f+g .
\end{array}\right.
\end{aligned}
$$

For any $\epsilon>0$, there exists $\delta=\delta\left(\epsilon, R_{0}, f\right)>0$ sufficiently small such that if

$$
\|g\|_{\infty}<\delta
$$

then

$$
\max _{0 \leq t \leq 1}\left\|\omega^{a}(t, \cdot)-\omega(t, \cdot)\right\|_{\infty}<\epsilon
$$

Proof of Lemma 6.6. By first taking $\|g\|_{\infty} \lesssim 1$, we have $\|f+g\|_{\infty} \lesssim f, R_{0} 1$. Since $\operatorname{supp}(f) \subset B\left(0, R_{0}\right)$ and $\operatorname{supp}(g) \subset B\left(0, R_{0}\right)$, we get

$$
\begin{aligned}
& \operatorname{supp}(\omega(t, \cdot)) \subset B\left(0, R_{1}\right), \\
& \operatorname{supp}\left(\omega^{a}(t, \cdot)\right) \subset B\left(0, R_{1}\right), \quad \forall 0 \leq t \leq 1,
\end{aligned}
$$

where $R_{1}>0$ is some constant depending on $R_{0}$ and $\|f\|_{\infty}$ only. 
Set $\eta=\omega^{a}-\omega$. Then $\eta$ satisfies the equation

$$
\left\{\begin{array}{l}
\partial_{t} \eta+\left(\Delta^{-1} \nabla^{\perp} \eta\right) \cdot \nabla \omega^{a}+u \cdot \nabla \eta=0 \\
\eta(0)=g
\end{array}\right.
$$

By a simple energy estimate, we have

$$
\max _{0 \leq t \leq 1}\left\|\nabla \omega^{a}(t, \cdot)\right\|_{\infty} \lesssim_{f} 1
$$

On the other hand, since $\operatorname{supp}(\eta(t, \cdot)) \subset B\left(0, R_{1}\right)$ for any $0 \leq t \leq 1$, we have

$$
\begin{aligned}
\left\|\Delta^{-1} \nabla^{\perp} \eta(t, \cdot)\right\|_{\infty} & \lesssim\|\eta(t, \cdot)\|_{1}+\|\eta(t, \cdot)\|_{\infty} \\
& \lesssim R_{1}\|\eta(t, \cdot)\|_{\infty} .
\end{aligned}
$$

By (6.45), we then get for any $0 \leq t \leq 1$,

$$
\|\eta(t, \cdot)\|_{\infty} \lesssim R_{1}, f\|g\|_{\infty}+\int_{0}^{t}\|\eta(s, \cdot)\|_{\infty} d s
$$

A Gronwall argument then yields

$$
\max _{0 \leq t \leq 1}\|\eta(t, \cdot)\|_{\infty} \lesssim R_{1}, f\|g\|_{\infty} .
$$

Therefore (6.44) follows by choosing $\|g\|_{\infty}$ sufficiently small.

We now sketch the

Proof of Theorem 1.6. We still choose the centers $z_{j}$ as in (6.33). The main modification is the choice of the initial profiles $h_{j}\left(\right.$ at $z_{j}$ ). For this we need to modify Lemma 6.4 and combine Lemma 6.6 so that each when a new profile $h_{J}, J \geq 2$ is added, besides satisfying the existing constraints (as specified in Lemma 6.4), the following also hold:

let $\omega^{(J)}$ be defined the same way as in (6.36), then $\left\|h_{J}\right\|_{\infty}$ is sufficiently small (as specified in Lemma 6.6) such that

$$
\max _{0 \leq t \leq 1}\left\|\omega^{(J)}(t, \cdot)-\omega^{(J-1)}(t, \cdot)\right\|_{\infty} \leq 2^{-J} .
$$

Note that by (6.37) we can always guarantee for some constant $R>0$ that

$$
\operatorname{supp}\left(\omega^{(J)}(t, \cdot)\right) \subset B(0, R), \quad \forall 0 \leq t \leq 1, J \geq 1 .
$$

We then view $\left(\omega^{(J)}\right)_{J \geq 1}$ as a Cauchy sequence in the Banach space $C_{t}^{0} C_{x}^{0}([0,1] \times$ $\overline{B(0, R)})$ and extracts the limit solution $\omega$ in the same space. By interpolation and Sobolev embedding, easy to check that $u^{(J)}=\Delta^{-1} \nabla^{\perp} \omega^{(J)}$ also forms a Cauchy sequence in $C_{t}^{0} L_{x}^{2} \cap C_{t}^{0} C_{x}^{\alpha}\left([0,1] \times \mathbb{R}^{2}\right)$ for any $0<\alpha<1$. Therefore $u^{(J)}$ converges to the limit $u=\Delta^{-1} \nabla^{\perp} \omega$ and $\omega$ is the desired solution. The other properties of $\omega$ can be proved in the same way as in Theorem 6.5. We omit the repetitive details. 


\section{3D EULER WITH NON-COMPACTLY SUPPORTED DATA}

The main building block in our construction for 3D Euler is the axisymmetric flow without swirl on $\mathbb{R}^{3}$. To unify the notation we first recall the definition and review some useful properties. We shall review some relevant literature along the way.

By an axisymmetric scalar function on $\mathbb{R}^{3}$, we mean a function of the form $f=f(r, z)$, with $x=\left(x_{1}, x_{2}, z\right), r=\sqrt{x_{1}^{2}+x_{2}^{2}}$. In other words the function is invariant under any rotation about the vertical axis $O Z=\{(0,0, z): z \in \mathbb{R}\}$. Analogously one can define an axisymmetric vector field on $\mathbb{R}^{3}$. The velocity field $u$ representing an axisymmetric flow on $\mathbb{R}^{3}$ generally takes the form

$$
u\left(x_{1}, x_{2}, z\right)=u^{r}(r, z) e_{r}+u^{\theta}(r, z) e_{\theta}+u^{z}(r, z) e_{z},
$$

where $\left(e_{r}, e_{\theta}, e_{z}\right)$ is the standard orthogonal basis for the cylindrical coordinate system:

$$
\begin{aligned}
e_{r} & =\frac{1}{r}\left(x_{1}, x_{2}, 0\right), \quad e_{\theta}=\frac{1}{r}\left(-x_{2}, x_{1}, 0\right), \\
e_{z} & =(0,0,1) .
\end{aligned}
$$

Here $u^{r}, u^{\theta}, u^{z}$ are called the radial, angular/swirl and axial velocity respectively. By an axisymmetric flow without swirl, we mean the angular (swirl) component $u^{\theta} \equiv 0$, that is

$$
u\left(x_{1}, x_{2}, z\right)=u^{r}(r, z) e_{r}+u^{z}(r, z) e_{z} .
$$

The corresponding vorticity $\omega=\nabla \times u$ then reduces to

$$
\omega\left(x_{1}, x_{2}, z\right)=\omega^{\theta}(r, z) e_{\theta}=\left(\partial_{z} u^{r}-\partial_{r} u^{z}\right) e_{\theta} .
$$

It follows easily that

$$
\begin{aligned}
(\omega \cdot \nabla) u & =\left(\omega^{\theta} e_{\theta} \cdot \nabla\right) u \\
& =\omega^{\theta} \frac{1}{r} u^{r} e_{\theta}=\frac{u^{r}}{r} \omega .
\end{aligned}
$$

Therefore the vorticity equation reads as

$$
\partial_{t} \omega+\left(u^{r} \partial_{r}+u^{z} \partial_{z}\right) \omega=\frac{1}{r} u^{r} \omega
$$

or simply,

$$
\partial_{t}\left(\frac{\omega}{r}\right)+(u \cdot \nabla)\left(\frac{\omega}{r}\right)=0 .
$$

In this way we obtain a transport equation for the quantity $\omega / r$. Since the velocity $u$ is divergence-free, all $L^{p}, 1 \leq p \leq \infty$ and similar Lorentz space norms of $\omega / r$ are preserved in time. These important conservation laws are the key to obtaining global solutions. Indeed Ukhovskii and Yudovich [26], and independently Ladyzhenskaya [19] first proved global wellposedness for initial velocity $u_{0} \in H^{1}$ with initial vorticity satisfying $\omega_{0} \in L^{2} \cap L^{\infty}, \frac{\omega_{0}}{r} \in L^{2} \cap L^{\infty}$. In terms of Sobolev regularity, one need the initial velocity $u_{0} \in H^{s}, s>7 / 2$ to have $\frac{1}{r} \omega_{0} \in L^{\infty}$. In 24], Shirota and Yanagisawa weakened the regularity on velocity to $u_{0} \in H^{s}$, $s>5 / 2$ which is the borderline in view of the $H^{s}$ local wellposedness theory in 3D. Danchin 9] showed global existence and uniqueness of solutions for initial vorticity $\omega_{0} \in L^{\infty} \cap L^{3,1}$ with $\frac{\omega}{r} \in L^{3,1}$ in bounded domains with $C^{2, \alpha}$ boundary or the 
whole space $\mathbb{R}^{3}$. In 1] Abidi, Hmidi and Keraan: 6 proved global wellposedness in the space $C_{t}^{0} B_{p, 1}^{1+\frac{3}{p}}\left(\mathbb{R}_{+} \times \mathbb{R}^{3}\right)$ for initial velocity $u_{0} \in B_{p}^{\frac{3}{p}+1}\left(\mathbb{R}^{3}\right), 1<p \leq \infty$ with the additional mild assumption that $\frac{\omega_{0}}{r} \in L^{3,1}\left(\mathbb{R}^{3}\right)$ and $\omega_{0} \in \widetilde{B}_{\infty, 1}^{0}$. Here $\widetilde{B}_{\infty, 1}^{0}$ is a subspace of $B_{\infty, 1}^{0}$ containing

$$
\left\{u \in \mathcal{S}^{\prime}\left(\mathbb{R}^{3}\right): \sum_{\substack{N \geq 2 \\ N \text { is dyadic }}}(\log N)\left\|P_{N} u\right\|_{\infty}<\infty\right\} .
$$

We now state a few basic lemmas needed for our construction later. Some of these are well-known facts which are already extensively used in the aforementioned works.

Lemma 7.1 ( $L^{p, q}$-preservation). Let $1 \leq p, q \leq \infty$. Suppose $u$ is a given smooth divergence-free vector field on $\mathbb{R}^{d}, d \geq 2$. Let $h$ be the smooth solution to the transport equation

$$
\left\{\begin{array}{l}
\partial_{t} h+(u \cdot \nabla) h=f \\
\left.h\right|_{t=0}=h_{0}
\end{array}\right.
$$

Then for any $t>0$, we have

$$
\|h(t)\|_{L^{p, q}\left(\mathbb{R}^{d}\right)} \leq\left\|h_{0}\right\|_{L^{p, q}\left(\mathbb{R}^{d}\right)}+\int_{0}^{t}\|f(\tau)\|_{L^{p, q}\left(\mathbb{R}^{d}\right)} d \tau .
$$

If $f \equiv 0$, then

$$
\|h(t)\|_{L^{p, q}\left(\mathbb{R}^{d}\right)}=\left\|h_{0}\right\|_{L^{p, q}\left(\mathbb{R}^{d}\right)} .
$$

Proof of Lemma 7.1. See for example Proposition 2 on p484 of Danchin 9] or Prop 2.2 of Abidi-Hmidi-Keraani [1. In the homogeneous case $f \equiv 0$, one just observes that $h(t)=h_{0} \circ \phi(t)$ where the flow map $\phi(t)$ is measure-preserving. This obviously implies $\left\|h_{0} \circ \phi(t)\right\|_{L^{p, q}}=\left\|h_{0}\right\|_{L^{p, q}}$ by using the definition of Lorentz spaces. Alternatively one can use $L^{p}$ conservation and interpolation, as done in [1. The general case of nonzero $f$ follows from Duhamel.

We shall use Lemma 7.1 often without explicit mentioning. The most useful case for us is the $L^{3,1}$ conservation of vorticity.

The next useful lemma is a Biot-Savart law estimate in the axisymmetric setting. It is the key to the proof of global wellposedness for the axisymmetric (without swirl) flow.

Lemma 7.2. There exists an absolute constant $C>0$ such that

$$
\left\|\frac{u^{r}}{r}\right\|_{L^{\infty}\left(\mathbb{R}^{3}\right)} \leq C\left\|\frac{\omega^{\theta}}{r}\right\|_{L^{3,1}\left(\mathbb{R}^{3}\right)},
$$

where $u=u^{r} e_{r}+u^{z} e_{z}, \omega=\nabla \times u=\omega^{\theta} e_{\theta}$.

\footnotetext{
${ }^{6}$ The work of Danchin 9 does not address the propagation of critical Besov regularity $B_{p, 1}^{\frac{3}{p}+1}$ due to the lack of Beale-Kato-Majda criteria in borderline Besov spaces.
} 
Proof of Lemma 7.2. See, for example, Proposition 3.1 of [1]. The key is to use Lemma 1 from [24] which gives the kernel estimate:

$$
\left|u^{r}(x)\right| \lesssim \int_{|y-x| \leq r} \frac{|\omega(y)|}{|x-y|^{2}} d y+r \int_{|y-x| \geq r} \frac{|\omega(y)|}{|x-y|^{3}} d y
$$

Occasionally we need the following lemma.

Lemma 7.3. Let $1<p<3$ and $u \in B_{p, q}^{\frac{3}{p}+1}\left(\mathbb{R}^{3}\right)$ be an axisymmetric divergence-free vector field. Let $\omega=\nabla \times u$ be its vorticity. Then

$$
\left\|\frac{\omega}{r}\right\|_{L^{3,1}\left(\mathbb{R}^{3}\right)} \lesssim\|u\|_{B_{p, 1}^{\frac{3}{p}+1}\left(\mathbb{R}^{3}\right)} .
$$

Proof of Lemma 7.3. This is Proposition 2.1 from [1]. the key is to prove $\left\|\frac{\omega}{r}\right\|_{L^{3,1}} \lesssim$ $\|\nabla \omega\|_{L^{3,1}}$ which is obtained by using $\omega(0,0, z) \equiv 0$ and the Fundamental Theorem of calculus. The embedding $B_{p, 1}^{\frac{3}{p}-1} \hookrightarrow L^{3,1}$ comes from interpolation

$$
\begin{aligned}
& \left(L^{p}, L^{r}\right)_{(\theta, 1)}=L^{3,1} \\
& \left(B_{p, 1}^{0}, B_{p, 1}^{\frac{3}{p}-\frac{3}{r}}\right)_{(\theta, 1)}=B_{p, 1}^{\theta\left(\frac{3}{p}-\frac{3}{r}\right)}=B_{p, 1}^{\frac{3}{p}-1},
\end{aligned}
$$

where $1<p<3<r<\infty$ and $\frac{1}{3}=\frac{1-\theta}{p}+\frac{\theta}{r}$, followed by the embedding of Besov into Lebesgue.

We now state a lemma which sets up some basic properties of the axisymmetric flow and the associated characteristic lines.

Lemma 7.4. Consider the Euler equation (in vorticity form)

$$
\left\{\begin{array}{l}
\partial_{t} \omega+u \cdot \nabla \omega=(\omega \cdot \nabla) u, \quad(t, x) \in[0, \infty) \times \mathbb{R}^{3}, \\
u=-\Delta^{-1} \nabla \times \omega, \\
\left.\omega\right|_{t=0}=\omega_{0},
\end{array}\right.
$$

where the initial vorticity $\omega_{0}=\nabla \times u_{0}$ and $u_{0}$ is a smooth axisymmetric velocity field without swirl.

Write

$$
u=u^{r} e_{r}+u^{z} e_{z},
$$

and consider the forward characteristic lines expressed in cylindrical coordinates, i.e.

$$
\left\{\begin{array}{l}
\partial_{t} \phi^{r}(t, r, z)=u^{r}\left(t, \phi^{r}(t, r, z), \phi^{z}(t, r, z)\right), \\
\partial_{t} \phi^{z}(t, r, z)=u^{z}\left(t, \phi^{r}(t, r, z), \phi^{z}(t, r, z)\right), \\
\left.\left(\phi^{r}, \phi^{z}\right)\right|_{t=0}=(r, z) .
\end{array}\right.
$$

Denote by $\tilde{\phi}(t, r, z)=\left(\tilde{\phi}^{r}(t, r, z), \tilde{\phi}^{z}(t, r, z)\right)$ the inverse map of $\phi=\left(\phi^{r}, \phi^{z}\right)$. Then the following hold:

- For any $z \in \mathbb{R}, t \geq 0$, we have

$$
\begin{aligned}
& u^{r}(t, 0, z)=0, \\
& \phi^{r}(t, 0, z)=0=\tilde{\phi}^{r}(t, 0, z) .
\end{aligned}
$$


Also

$$
\phi^{r}(t, r, z)>0, \tilde{\phi}^{r}(t, r, z)>0, \quad \forall r>0, z \in \mathbb{R}, t \geq 0 .
$$

- For any $t \geq 0, r>0, z \in \mathbb{R}$, we have

$$
\begin{aligned}
& \operatorname{det}\left(\frac{\partial\left(\phi^{r}(t), \phi^{z}(t)\right)}{\partial(r, z)}\right) \\
= & \frac{r}{\phi^{r}(t, r, z)} \\
= & \exp \left(-\int_{0}^{t} \frac{1}{\phi^{r}(s, r, z)} u^{r}\left(s, \phi^{r}(s, r, z), \phi^{z}(s, r, z)\right) d s\right) .
\end{aligned}
$$

For $t \geq 0, r=0, z \in \mathbb{R}$, we have

$$
\begin{aligned}
& \left.\operatorname{det}\left(\frac{\partial\left(\phi^{r}(t), \phi^{z}(t)\right)}{\partial(r, z)}\right)\right|_{(r, z)=(0, z)} \\
= & \lim _{r \rightarrow 0} \frac{r}{\phi^{r}(t, r, z)} \\
= & \exp \left(-\int_{0}^{t} \lim _{r \rightarrow 0} \frac{1}{\phi^{r}(s, r, z)} u^{r}\left(s, \phi^{r}(s, r, z), \phi^{z}(s, r, z)\right) d s\right) .
\end{aligned}
$$

Here both limits exist and are finite.

- Similarly for any $t \geq 0, r \geq 0, z \in \mathbb{R}$, we have

$$
\operatorname{det}\left(\frac{\partial\left(\tilde{\phi}^{r}(t), \tilde{\phi}^{z}(t)\right)}{\partial(r, z)}\right)=\frac{r}{\tilde{\phi}^{r}(t, r, z)} .
$$

Proof of Lemma 7.4. We first show (7.3). Obviously by (7.1), we must have

$$
u^{r}(t, 0, z)=0, \quad \forall t \geq 0, z \in \mathbb{R},
$$

since otherwise $u$ would not be smooth at $x=(0,0, z)$. By a similar consideration, we can Taylor expand $\left(u_{1}, u_{2}\right)$ around the point $(0,0, z)$ to get

$$
\begin{aligned}
\left(u_{1}\left(t, x_{1}, x_{2}, z\right), u_{2}\left(t, x_{1}, x_{2}, z\right)\right) & =c(t, z)\left(x_{1}, x_{2}\right)+O\left(r^{2}\right) \\
& =c(t, z) r e_{r}+O\left(r^{2}\right) \\
& =u^{r}(t, r, z) e_{r} .
\end{aligned}
$$

Here the coefficient $c(t, z)$ is scalar valued and

$$
c(t, z)=\left(\partial_{1} u_{1}\right)(t, 0,0, z)=\left(\partial_{2} u_{2}\right)(t, 0,0, z) .
$$

Therefore

$$
u^{r}(t, r, z)=c(t, z) r+O\left(r^{2}\right)
$$

and

$$
\begin{aligned}
& \lim _{r \rightarrow 0} \frac{u^{r}(t, r, z)}{r}=c(t, z), \\
& \lim _{r \rightarrow 0}\left(\partial_{r} u^{r}\right)(t, r, z)=c(t, z) .
\end{aligned}
$$

By (7.2), we have

$$
\begin{aligned}
\frac{d}{d t} \phi^{r} & =u^{r}\left(t, \phi^{r}, \phi^{z}\right)-u^{r}\left(t, 0, \phi^{z}\right) \\
& =\left(\int_{0}^{1}\left(\partial_{r} u^{r}\right)\left(t, \theta \phi^{r}, \phi^{z}\right) d \theta\right) \phi^{r} .
\end{aligned}
$$


Integrating in time then gives

$$
\phi^{r}(t, r, z)=r \exp \left(\int_{0}^{t} \int_{0}^{1}\left(\partial_{r} u^{r}\right)\left(s, \theta \phi^{r}(s, r, z), \phi^{z}(s, r, z)\right) d \theta d s\right) .
$$

Clearly (7.4) follows. Also

$$
\phi^{r}(t, 0, z)=0, \quad \text { for any } t \geq 0, z \in \mathbb{R} .
$$

Next we show (7.5). We shall calculate $\operatorname{det}\left(\frac{\partial\left(\phi^{r}, \phi^{z}\right)}{\partial(r, z)}\right)$ in two ways which in turn would yield the first and second identity in (7.5).

Introduce new variables

$$
\begin{aligned}
R & :=\frac{1}{2} \phi^{r}(t, r, z)^{2}, \\
Z & :=\phi^{z}(t, r, z) .
\end{aligned}
$$

Then

$$
\left\{\begin{array}{l}
\frac{d}{d t} R=V^{r}(t, R, Z) \\
\frac{d}{d t} Z=V^{z}(t, R, Z) \\
\left.(R, Z)\right|_{t=0}=\left(R_{0}, Z_{0}\right)
\end{array}\right.
$$

Here

$$
\begin{aligned}
& V^{r}(t, R, Z):=\sqrt{2 R} u^{r}(t, \sqrt{2 R}, Z), \\
& V^{z}(t, R, Z):=u^{z}(t, \sqrt{2 R}, Z) .
\end{aligned}
$$

By the incompressibility condition $\nabla \cdot u=0$, we have

$$
\frac{1}{r} \partial_{r}\left(r u^{r}(t, r, z)\right)+\partial_{z} u^{z}(t, r, z)=0 .
$$

Therefore easy to check that

$$
\partial_{R} V^{r}(t, R, Z)+\partial_{Z} V^{z}(t, R, Z)=0 .
$$

It follows that

$$
\operatorname{det}\left(\frac{\partial(R(t), Z(t))}{\partial\left(R_{0}, Z_{0}\right)}\right)=1, \quad \forall t \geq 0
$$

or

$$
\frac{\partial R}{\partial R_{0}} \frac{\partial Z}{\partial Z_{0}}-\frac{\partial R}{\partial Z_{0}} \frac{\partial Z}{\partial R_{0}}=1 .
$$

Letting $R_{0}=\frac{1}{2} r^{2}, Z_{0}=z$ and using (7.10) then gives

$$
\operatorname{det}\left(\frac{\partial\left(\phi^{r}, \phi^{z}\right)}{\partial(r, z)}\right)=\frac{r}{\phi^{r}}
$$

For the second way of calculating $\operatorname{det}\left(\frac{\partial\left(\phi^{r}, \phi^{z}\right)}{\partial(r, z)}\right)$, we need to use the following elementary fact: if $A(t) \in \mathbb{R}^{d \times d}, B(t) \in \mathbb{R}^{d \times d}$ are smooth matrix-valued functions solving the ODE

$$
\frac{d}{d t} A(t)=B(t) A(t)
$$


with $A(0)=I_{d}$ (the identity matrix on $\mathbb{R}^{d \times d}$ ), then

$$
\operatorname{det}(A(t))=\exp \left(\int_{0}^{t} \operatorname{tr}(B(s)) d s\right)
$$

where $\operatorname{tr}(\cdot)$ denotes the usual matrix trace.

By (7.2), we have

$$
\frac{d}{d t}\left(\begin{array}{ll}
\frac{\partial \phi^{r}}{\partial r_{z}} & \frac{\partial \phi^{r}}{\partial z_{z}} \\
\frac{\partial \phi^{z}}{\partial r} & \frac{\partial \phi^{z}}{\partial z}
\end{array}\right)=\left(\begin{array}{ll}
\partial_{r} u^{r} & \partial_{z} u^{r} \\
\partial_{r} u^{z} & \partial_{z} u^{z}
\end{array}\right)\left(\begin{array}{ll}
\frac{\partial \phi^{r}}{\partial r_{z}} & \frac{\partial \phi^{r}}{\partial z_{z}} \\
\frac{\partial \phi^{z}}{\partial r} & \frac{\partial \phi^{z}}{\partial z}
\end{array}\right) .
$$

Since $\partial_{r} u^{r}+\partial_{z} u^{z}=-\frac{1}{r} u^{r}$, we get

$$
\begin{aligned}
& \left(\partial_{r} u^{r}\right)\left(t, \phi^{r}, \phi^{z}\right)+\left(\partial_{z} u^{z}\right)\left(t, \phi^{r}, \phi^{z}\right) \\
= & -\frac{1}{\phi^{r}} u^{r}\left(t, \phi^{r}, \phi^{z}\right) .
\end{aligned}
$$

Therefore the second identity in (7.5) follows. It is not difficult to check that this also coincides with (7.9) derived earlier.

The existence of the limits (7.6) is a simple consequence of (7.8) and (7.9).

Finally (7.7) follows easily from (7.5) and the identity

$$
D \tilde{\phi}=((D \phi)(\tilde{\phi}))^{-1} .
$$

We now take a parameter $A \gg 1$ and define (by a slight abuse of notation) a class of axisymmetric functions

$$
\begin{aligned}
g_{A}\left(x_{1}, x_{2}, z\right) & =g_{A}(r, z) \\
& =\frac{\sqrt{\log A}}{A} \sum_{A \leq k \leq 2 A} \eta_{k}(r, z),
\end{aligned}
$$

where $r=\sqrt{x_{1}^{2}+x_{2}^{2}}$ and

$$
\eta_{k}(r, z)=\sum_{\epsilon_{1}= \pm 1} \epsilon_{1} \eta_{0}\left(\frac{\left(r-2^{-k}, z-\epsilon_{1} 2^{-k}\right)}{2^{-(k+10)}}\right) .
$$

Here we choose $\eta_{0} \in C_{c}^{\infty}(B(0,1))$ to be a radial compactly supported function such that $0 \leq \eta_{0} \leq 1$. Note that by construction $\eta_{k}$ is an odd function of $z$ and so is $g_{A}$. Also it is easy to see that

$$
\begin{aligned}
\eta_{k}\left(2^{-k} r, 2^{-k} z\right) & =\sum_{\epsilon_{1}= \pm 1} \epsilon_{1} \eta_{0}\left(\frac{\left(r-1, z-\epsilon_{1}\right)}{2^{-10}}\right) \\
& =: \tilde{\eta}_{0}(r, z)
\end{aligned}
$$


Recall $e_{\theta}=\frac{1}{r}\left(-x_{2}, x_{1}, 0\right)$. We first check that

$$
\begin{aligned}
&\left\|g_{A} e_{\theta}\right\|_{L^{\infty}\left(\mathbb{R}^{3}\right)} \lesssim \frac{\sqrt{\log A}}{A} . \\
&\left\|g_{A} e_{\theta}\right\|_{\dot{B}_{2,1}^{\frac{3}{2}}\left(\mathbb{R}^{3}\right)} \lesssim \sqrt{\log A} . \\
&\left\|\frac{g_{A}}{r} e_{\theta}\right\|_{L^{3,1}\left(\mathbb{R}^{3}\right)} \lesssim \sqrt{\log A} . \\
&\left\|g_{A}\right\|_{\dot{H}^{\frac{3}{2}\left(\mathbb{R}^{3}\right)}} \lesssim \frac{\sqrt{\log A}}{\sqrt{A}} \\
&\left\|g_{A} e_{\theta}\right\|_{\dot{H}^{\frac{3}{2}\left(\mathbb{R}^{3}\right)}} \lesssim \frac{\sqrt{\log A}}{\sqrt{A}} .
\end{aligned}
$$

Note that (7.14) is obvious. The property (7.15) follows from the triangle inequality and the fact that each $\eta_{k} e_{\theta}$ has the same $\dot{B}_{2,1}^{\frac{3}{2}}$ norm. The inequality (7.16) follows from Lemma 7.3 .

For (7.17), albeit standard, we explain how to check it. By definition and direct expansion, we have

$$
\begin{aligned}
& \left\|g_{A}\right\|_{\dot{H}^{\frac{3}{2}}}^{2} \\
= & \frac{\log A}{A^{2}} \sum_{A \leq k \leq 2 A} \sum_{A \leq l \leq 2 A} \int_{\mathbb{R}^{3}}|\nabla|^{\frac{3}{2}} \eta_{k} \cdot|\nabla|^{\frac{3}{2}} \eta_{l} d x .
\end{aligned}
$$

Therefore it suffices to show for each $k$,

$$
\left.\sum_{A \leq l \leq 2 A}\left|\int_{\mathbb{R}^{3}}\right| \nabla\right|^{\frac{3}{2}} \eta_{k} \cdot|\nabla|^{\frac{3}{2}} \eta_{l} d x \mid \lesssim 1
$$

By scaling $(r, z) \rightarrow\left(2^{-k} r, 2^{-k} z\right)$ and (7.13), we have

$$
\text { LHS of }\left.(17.19) \lesssim \sum_{l \in \mathbb{Z}}\left|\int_{\mathbb{R}^{3}}\right| \nabla\right|^{\frac{3}{2}} \tilde{\eta}_{0} \cdot|\nabla|^{\frac{3}{2}} \eta_{l} d x \mid
$$

where $\tilde{\eta}_{0}$ was defined in (7.13). Note that $\tilde{\eta}_{0} \in C_{c}^{\infty}\left(\mathbb{R}^{3}\right)$ since it is supported on $r \sim 1,|z| \sim 1$.

Now discuss two cases. If $l \geq 0$, then

$$
\begin{aligned}
& \left.\left|\int_{\mathbb{R}^{3}}\right| \nabla\right|^{\frac{3}{2}} \tilde{\eta}_{0} \cdot|\nabla|^{\frac{3}{2}} \eta_{l} d x \mid \\
= & \left.\left|\int_{\mathbb{R}^{3}}\right| \nabla\right|^{3} \tilde{\eta}_{0} \cdot \eta_{l} d x \mid \\
\lesssim & \left\|\left.|| \nabla\right|^{3} \tilde{\eta}_{0}\right\|_{L^{\infty}} \cdot\left\|\eta_{l}\right\|_{L^{1}} \lesssim 2^{-3 l},
\end{aligned}
$$

which is summable for $l \geq 0$. 
On the other hand if $l<0$, then

$$
\begin{aligned}
& \left.\left|\int_{\mathbb{R}^{3}}\right| \nabla\right|^{\frac{3}{2}} \tilde{\eta}_{0} \cdot|\nabla|^{\frac{3}{2}} \eta_{l} d x \mid \\
= & \left|\int_{\mathbb{R}^{3}}\right| \nabla\left|\tilde{\eta}_{0} \cdot \Delta \eta_{l} d x\right| \\
\lesssim & \leqslant|| \nabla \mid \tilde{\eta}_{0}\left\|_{L^{2}} \cdot\right\| \Delta \eta_{l} \|_{L^{2}} \\
\lesssim & \lesssim 2^{\frac{1}{2} l}
\end{aligned}
$$

which is also summable for $l<0$.

Therefore (7.19) follows and (7.17) is proved.

For (7.18), we note that by (7.13),

$$
\begin{aligned}
& \eta_{k}(r, z) e_{\theta} \\
= & \tilde{\eta}_{0}\left(2^{k} r, 2^{k} z\right) \frac{1}{2^{k} r}\left(-2^{k} x_{2}, 2^{k} x_{1}, 0\right) \\
= & \eta_{\mathrm{vec}}\left(2^{k} x\right)
\end{aligned}
$$

where

$$
\eta_{\mathrm{vec}}(x)=\eta_{\mathrm{vec}}\left(x_{1}, x_{2}, z\right)=\tilde{\eta}_{0}(r, z) \frac{1}{r}\left(-x_{2}, x_{1}, 0\right) .
$$

Since by definition $\tilde{\eta}_{0}$ is supported on $r \sim 1, \eta_{\text {vec }}$ is a smooth function. Therefore (7.18) can be proved in the same way as (7.17) (note that only scaling is used in the argument).

Lemma 7.5. For any smooth axisymmetric $f$ on $\mathbb{R}^{3}$ (i.e. $f=f(x)=f(r, z)$, $\left.x=\left(x_{1}, x_{2}, z\right), r=\sqrt{x_{1}^{2}+x_{2}^{2}}\right)$, we have

$$
\begin{aligned}
& \left(\partial_{z}\left(\Delta-\frac{1}{r^{2}}\right)^{-1} \partial_{z} f\right)(r, z) \\
= & \left(\partial_{z z}\left(\Delta-\frac{1}{r^{2}}\right)^{-1} f\right)(r, z) \\
= & C \cdot \int_{\mathbb{R}^{5}} K(x-y) \cdot \frac{\left|x^{\prime}\right|}{\left|y^{\prime}\right|} f(y) d y, \quad x=(r, 0,0,0, z),
\end{aligned}
$$

where $C>0$ is an absolute constant, $x^{\prime}=\left(x_{1}, \cdots, x_{4}\right), y^{\prime}=\left(y_{1}, \cdots, y_{4}\right)$, and

$$
K(\tilde{x})=\frac{\left|\tilde{x}^{\prime}\right|^{2}-4 \tilde{x}_{5}^{2}}{|\tilde{x}|^{7}}+\frac{1}{5 C} \delta(\tilde{x}) .
$$

Here $\delta(\cdot)$ is the Dirac delta function on $\mathbb{R}^{5}$. On the RHS of (7.20) we naturally regard $f$ as an axisymmetric function on $\mathbb{R}^{5}$ with the identification $f(y)=f\left(\left|y^{\prime}\right|, y_{5}\right)$. Also by axisymmetry the RHS of (7.20) depends only on $\left(\left|x^{\prime}\right|, x_{5}\right)$ so that we can actually choose any $x$ with $\left|x^{\prime}\right|=r, x_{5}=z$.

Similarly we have

$$
\begin{aligned}
& \left(\partial_{r}\left(\Delta-\frac{1}{r^{2}}\right)^{-1} \partial_{z} f\right)(r, z) \\
= & C \cdot \int_{\mathbb{R}^{5}}\left(\frac{1}{|x-y|^{5}}-5 r \cdot \frac{r-y_{1}}{|x-y|^{7}}\right) \cdot \frac{x_{5}-y_{5}}{\left|y^{\prime}\right|} f(y) d y, \quad x=(r, 0,0,0, z) .
\end{aligned}
$$


Proof of Lemma 7.5. We first compute the kernel $\left(\Delta-\frac{1}{r^{2}}\right)^{-1}$. To derive the explicit representation, consider the equation

$$
\left(\Delta-\frac{1}{r^{2}}\right) u=f
$$

or

$$
\left(\partial_{r r}+\frac{1}{r} \partial_{r}-\frac{1}{r^{2}}+\partial_{z z}\right) u=f .
$$

Set $u=r v$. Then

$$
\begin{aligned}
\Delta(r v) & =r \Delta v+2\left(\partial_{r} r\right) \partial_{r} v+v \Delta(r) \\
& =r \Delta v+2 \partial_{r} v+\frac{1}{r} v \\
& =r \Delta_{5} v+\frac{1}{r} v
\end{aligned}
$$

where

$$
\Delta_{5}=\partial_{r r}+\frac{3}{r} \partial_{r}+\partial_{z z}
$$

is the five dimensional Laplacian in cylindrical coordinates.

Therefore

$$
\left(\Delta-\frac{1}{r^{2}}\right) u=\left(\Delta-\frac{1}{r^{2}}\right)(r v)=r \Delta_{5} v
$$

and we only need to solve

$$
\Delta_{5} v=\frac{1}{r} f
$$

Inverting the Laplacian then gives

$$
v=C \cdot \int_{\mathbb{R}^{5}} \frac{-1}{|x-y|^{3}} \cdot \frac{1}{\left|y^{\prime}\right|} f(y) d y
$$

and obviously

$$
u(x)=C \cdot \int_{\mathbb{R}^{5}} \frac{-1}{|x-y|^{3}} \cdot \frac{\left|x^{\prime}\right|}{\left|y^{\prime}\right|} f(y) d y .
$$

Note that in the above expression we have pure convolution in the $y_{5}$ variable. Therefore the first equality in (7.20) hold. Differentiating in $z=x_{5}$ variable twice then gives (7.20). In a similar way we can derive (7.22).

Lemma 7.6. Let $\phi=\phi(r, z)=\left(\phi^{r}(r, z), \phi^{z}(r, z)\right)$ be a bi-Lipschitz map on $[0, \infty) \times$ $\mathbb{R}$ such that the following hold:

- for any $r \geq 0, z \in \mathbb{R}$,

$$
\phi^{r}(0, z)=0 \quad \text { and } \phi^{z}(r, 0)=0 ;
$$

- for some integer $n_{0} \geq 1$,

$$
\|D \phi\|_{\infty}+\|D \tilde{\phi}\|_{\infty} \leq 2^{n_{0}},
$$

where $\tilde{\phi}$ is the inverse map of $\phi$. 
Define

$$
w(r, z)=\left(T \omega_{0}\right)(r, z)=\frac{\omega_{0}(\phi(r, z))}{\phi^{r}(r, z)} r,
$$

where $\omega_{0}=g_{A}$ and $g_{A}$ is defined in (7.11).

Then

$$
\left\|\partial_{z z}\left(\Delta-\frac{1}{r^{2}}\right)^{-1} \omega\right\|_{\infty} \leq C \cdot 2^{2 n_{0}} \cdot \frac{\sqrt{\log A}}{A},
$$

where $C>0$ is an absolute constant.

If in addition the map $\phi$ preserves the measure $r d r d z$, i.e.

$$
\frac{\phi^{r}}{r} \operatorname{det}\left(\frac{\partial\left(\phi^{r}, \phi^{z}\right)}{\partial(r, z)}\right) \equiv 1 ;
$$

and $\phi$ is odd in the $z$-variable, i.e.

$$
\begin{aligned}
& \phi^{r}(r,-z)=\phi^{r}(r, z), \quad \forall r \geq 0, z \in \mathbb{R} ; \\
& \phi^{z}(r,-z)=-\phi^{z}(r, z), \quad \forall r \geq 0, z \in \mathbb{R} ;
\end{aligned}
$$

then for some absolute constant $C_{1}>0$,

$$
\begin{aligned}
& -\left(\partial_{r}\left(\Delta-\frac{1}{r^{2}}\right)^{-1} \partial_{z} w\right)(0,0) \\
\geq & C_{1} \cdot \sqrt{\log A} \cdot 2^{-8 n_{0}} .
\end{aligned}
$$

Remark 7.7. In the proof of (7.26) below, we do not use the odd symmetry in the $z$-variable of the function $g_{A}$. By itself the axisymmetry is enough to control the singular operator $\partial_{z z}\left(\Delta-\frac{1}{r^{2}}\right)^{-1}$.

Remark 7.8. For our application later, $\omega$ actually correspond to $\omega^{\theta}$, and the expression $-\left(\Delta-\frac{1}{r^{2}}\right)^{-1} \partial_{z} \omega$ in (17.27) will correspond to the radial velocity $u^{r}$, see (17.36).

Proof of Lemma 7.6. We shall adopt the same notations as in Lemma 7.5. By (7.23) and (7.24), it is not difficult to check that if $r \sim 2^{-m}$ and $|z| \sim 2^{-m}$ for some integer $m$, then

$$
\begin{aligned}
2^{-m-n_{0}} \lesssim \phi^{r}(r, z) & \lesssim 2^{-m+n_{0}}, \\
2^{-m-n_{0}} \lesssim\left|\phi^{z}(r, z)\right| & \lesssim 2^{-m+n_{0}} .
\end{aligned}
$$

Similarly if $\phi^{r}(r, z) \sim 2^{-m},\left|\phi^{z}(r, z)\right| \sim 2^{-m}$, then

$$
\begin{aligned}
2^{-m-n_{0}} & \lesssim r \\
2^{-m-n_{0}} \lesssim|z| & \lesssim 2^{-m+n_{0}}, \\
& \lesssim 2^{-m+n_{0}} .
\end{aligned}
$$

These facts will be used below.

By (7.25) and (7.11), we have

$$
\begin{aligned}
& \partial_{z z}\left(\Delta-\frac{1}{r^{2}}\right)^{-1} \omega \\
= & \frac{\sqrt{\log A}}{A} \sum_{A \leq k \leq 2 A}\left(\partial_{z z}\left(\Delta-\frac{1}{r^{2}}\right)^{-1}\right)\left(T \eta_{k}\right) .
\end{aligned}
$$

We shall estimate each piece $\partial_{z z}\left(\Delta-\frac{1}{r^{2}}\right)^{-1}\left(T \eta_{k}\right)$. 
By (7.20) and (7.21), we write

$$
\begin{aligned}
& \left|\left(\partial_{z z}\left(\Delta-\frac{1}{r^{2}}\right)^{-1}\left(T \eta_{k}\right)\right)(x)\right| \\
& \lesssim\left|\int_{\mathbb{R}^{5}} K_{1}(x-y)\right| x^{\prime}\left|\frac{\eta_{k}\left(\phi\left(\left|y^{\prime}\right|, y_{5}\right)\right)}{\phi^{r}\left(\left|y^{\prime}\right|, y_{5}\right)} d y\right| \\
& \quad+r \frac{\eta_{k}(\phi(r, z))}{\phi^{r}(r, z)}
\end{aligned}
$$

where

$$
K_{1}(\tilde{x})=\frac{\left|\tilde{x}^{\prime}\right|^{2}-4 \tilde{x}_{5}^{2}}{|\tilde{x}|^{7}} .
$$

The contribution of (7.29) is of no problem for us. Indeed on the support of $\eta_{k}(\phi(r, z))$, we have

$$
\phi^{r}(r, z) \sim 2^{-k}, \quad \text { and }\left|\phi^{z}(r, z)\right| \sim 2^{-k} .
$$

Therefore $2^{-k-n_{0}} \lesssim r \lesssim 2^{-k+n_{0}}$ and

$$
\frac{r}{\phi^{r}(r, z)} \lesssim 2^{n_{0}}
$$

Since the supports of the $\eta_{k}$ functions are mutually disjoint, it follows that

$$
\sum_{A \leq k \leq 2 A} r \frac{\eta_{k}(\phi(r, z))}{\phi^{r}(r, z)} \lesssim 2^{n_{0}}
$$

Hence we only need to consider the contribution of (7.28) to (7.26). By the same consideration as before we have in (7.28) the integration variable $y$ is localized to the regime:

$$
\begin{aligned}
& 2^{-k-n_{0}} \lesssim\left|y^{\prime}\right| \lesssim 2^{-k+n_{0}}, \\
& 2^{-k-n_{0}} \lesssim\left|y_{5}\right| \lesssim 2^{-k+n_{0}}, \\
& \left|\phi\left(\left|y^{\prime}\right|, y_{5}\right)\right| \sim 2^{-k}, \quad \text { and } \phi^{r}\left(\left|y^{\prime}\right|, y_{5}\right) \sim 2^{-k} .
\end{aligned}
$$

Obviously if $x=0$, then due to the factor $\left|x^{\prime}\right|=0$, the integral (7.28) also vanishes. Therefore we only need to consider the case $x \neq 0$.

Assume $|x| \sim 2^{-l}$. We discuss two cases.

Case 1: $2^{k} \gg 2^{l+n_{0}}$. In this case $|x| \gg|y|\left(\right.$ recall $\left.|y| \lesssim 2^{-k+n_{0}}\right)$ and $|x-y| \sim|x|$. Therefore

$$
\begin{aligned}
(7.28) & \lesssim\left\|K_{1}(y)\right\|_{L^{\infty}\left(|y| \sim 2^{-l}\right)} \cdot 2^{-l} \cdot \frac{\operatorname{Leb}\left\{y \in \mathbb{R}^{5}: 2^{-k-n_{0}} \lesssim|y| \lesssim 2^{-k+n_{0}}\right\}}{2^{-k}} \\
& \lesssim 2^{5 l} \cdot 2^{-l} \cdot \frac{\left(2^{-k+n_{0}}\right)^{5}}{2^{-k}} \\
& \lesssim 2^{-4 k+4 l+5 n_{0}} .
\end{aligned}
$$

Case 2: $2^{k} \lesssim 2^{l+n_{0}}$. In this case we note that the kernel $K_{1}$ in (7.28) corresponds to a Riesz-type operator on $\mathbb{R}^{5}$. By using the interpolation inequality

$$
\left\|\mathcal{R}_{i j} f\right\|_{L^{\infty}\left(\mathbb{R}^{5}\right)} \lesssim\|f\|_{L^{5}\left(\mathbb{R}^{5}\right)}^{\frac{1}{2}} \cdot\|\nabla f\|_{L^{\infty}\left(\mathbb{R}^{5}\right)}^{\frac{1}{2}},
$$


we can bound (7.28) as

$$
\begin{aligned}
&(17.28) \lesssim 2^{-l} \cdot\left|\int_{\mathbb{R}^{5}} K_{1}(x-y) \cdot \frac{\eta_{k}\left(\phi\left(\left|y^{\prime}\right|, y_{5}\right)\right)}{\phi^{r}\left(\left|y^{\prime}\right|, y_{5}\right)} d y\right| \\
& \lesssim 2^{-l} \cdot\left\|\frac{1}{2^{-k}}\right\|_{L^{5}\left(y \in \mathbb{R}^{5}: 2^{-k-n_{0}} \lesssim|y| \lesssim 2^{-k+n_{0}}\right)}^{\frac{1}{2}} \\
& \cdot\left(\left\|\partial_{r}\left(\frac{\eta_{k}(\phi(r, z))}{\phi^{r}(r, z)}\right)\right\|_{\infty}+\left\|\partial_{z}\left(\frac{\eta_{k}(\phi(r, z))}{\phi^{r}(r, z)}\right)\right\|_{\infty}\right)^{\frac{1}{2}} \\
& \lesssim 2^{-l} \cdot 2^{\frac{k}{2}} \cdot\left(2^{-k+n_{0}}\right)^{\frac{1}{2}} \cdot\left(2^{n_{0}} \cdot 2^{2 k}\right)^{\frac{1}{2}} \\
& \lesssim 2^{n_{0}-l+k} .
\end{aligned}
$$

Collecting the estimates, we have

$$
\begin{aligned}
\sum_{A \leq k \leq 2 A}\left(\frac{(7.28)}{}\right. & \lesssim \sum_{2^{k} \gg 2^{l+n_{0}}} 2^{-4 k+4 l+5 n_{0}}+\sum_{2^{k} \lesssim 2^{l+n_{0}}} 2^{n_{0}-l+k} \\
& \lesssim 2^{2 n_{0}} .
\end{aligned}
$$

Hence the estimate (7.26) follows.

By (7.22) and parity of $\phi$ and $\eta_{k}$, we have

$$
\begin{aligned}
& -\left(\partial_{r}\left(\Delta-\frac{1}{r^{2}}\right)^{-1} \partial_{z} \omega\right)(0,0) \\
= & C \int_{\mathbb{R}^{5}} \frac{y_{5}}{|y|^{5}} \cdot \frac{1}{\left|y^{\prime}\right|} \omega(y) d y \\
= & C \int_{\mathbb{R}^{5}} \frac{y_{5}}{|y|^{5}} \cdot \frac{\omega_{0}\left(\phi\left(\left|y^{\prime}\right|, y_{5}\right)\right)}{\phi^{r}\left(\left|y^{\prime}\right|, y_{5}\right)} d y \\
= & C \frac{\sqrt{\log A}}{A} \cdot \sum_{A \leq k \leq 2 A} \int_{\mathbb{R}^{5}} \frac{y_{5}}{|y|^{5}} \cdot \frac{\eta_{k}\left(\phi\left(\left|y^{\prime}\right|, y_{5}\right)\right)}{\phi^{r}\left(\left|y^{\prime}\right|, y_{5}\right)} d y \\
= & 2 C \frac{\sqrt{\log A}}{A} \cdot \sum_{A \leq k \leq 2 A} \int_{y \in \mathbb{R}^{5}: y_{5}>0} \frac{y_{5}}{|y|^{5}} \cdot \frac{\eta_{k}\left(\phi\left(\left|y^{\prime}\right|, y_{5}\right)\right)}{\phi^{r}\left(\left|y^{\prime}\right|, y_{5}\right)} d y \\
\gtrsim & \frac{\sqrt{\log A}}{A} \sum_{A \leq k \leq 2 A} \frac{2^{-k-n_{0}}}{\left(2^{-k+n_{0}}\right)^{5}} \cdot \frac{1}{2^{-k}} \cdot \int \eta_{k}(\phi(r, z)) r^{3} d r d z .
\end{aligned}
$$

Since the map $\phi$ preserves the measure $r d r d z$, the inverse map $\tilde{\phi}$ also preserves the same measure. By the change of variable $(r, z) \rightarrow \tilde{\phi}(r, z)$, we have

$$
\begin{aligned}
& \int \eta_{k}(\phi(r, z)) r^{3} d r d z \\
= & \int \eta_{k}(r, z) \tilde{\phi}^{r}(r, z)^{2} r d r d z \\
\gtrsim & \left(2^{-k-n_{0}}\right)^{2}\left\|\eta_{k}\right\|_{L^{1}\left(\mathbb{R}^{3}\right)} \\
\gtrsim & 2^{-5 k-2 n_{0}} .
\end{aligned}
$$


Plugging this estimate into (7.30), we obtain

$$
\begin{aligned}
&-\left(\partial_{r}\left(\Delta-\frac{1}{r^{2}}\right)^{-1} \partial_{z} \omega\right)(0,0) \\
& \gtrsim \frac{\sqrt{\log A}}{A} \sum_{A \leq k \leq 2 A} 2^{-8 n_{0}} \\
& \gtrsim \sqrt{\log A} \cdot 2^{-8 n_{0}} .
\end{aligned}
$$

Hence (7.27) is proved.

We now prove the existence of large deformation in the 3D axisymmetric case. To fix the notation, consider the 3D axisymmetric Euler equation without swirl in simplified form

$$
\left\{\begin{array}{l}
\partial_{t}\left(\frac{\omega^{\theta}}{r}\right)+(u \cdot \nabla)\left(\frac{\omega^{\theta}}{r}\right)=0, \quad(r, z) \in(0, \infty) \times \mathbb{R}, 0<t \leq 1, \\
\left.\omega^{\theta}\right|_{t=0}=g_{A},
\end{array}\right.
$$

where $u=u^{r} e_{r}+u^{z} e_{z}$ and $g_{A}$ is defined in (7.11). Note that here $\omega^{\theta}$ is a scalarvalued function which is related to $u$ by the relation $\operatorname{curl}(u)=\omega^{\theta} e_{\theta}$.

Let $\phi=\phi(t, r, z)$ be the forward characteristic line as defined in (17.2) and $\tilde{\phi}=$ $\tilde{\phi}(t, r, z)$ be the inverse map. Then

Proposition 7.9. For all A sufficiently large, we have

$$
\max _{0 \leq t \leq \frac{1}{\log \log A}}\left(\|D \phi(t)\|_{\infty}+\|D \tilde{\phi}(t)\|_{\infty}\right) \geq \log \log A .
$$

Proof of Proposition 7.9. We argue by contradiction. Assume

$$
\max _{0 \leq t \leq \frac{1}{B}}\left(\|D \phi(t)\|_{\infty}+\|D \tilde{\phi}(t)\|_{\infty}\right) \leq B
$$

where for simplicity of the notation we denote $B=\log \log A$.

Denote $D(t)=D(t, r, z)=(D \phi)(t, r, z)$. By (7.2), we have

$$
\begin{aligned}
\partial_{t} D(t) & =\left(\begin{array}{ll}
\partial_{r} u^{r} & \partial_{z} u^{r} \\
\partial_{r} u^{z} & \partial_{z} u^{z}
\end{array}\right) D(t) \\
& =\left(\begin{array}{cr}
\partial_{r} u^{r} & 0 \\
0 & \partial_{z} u^{z}
\end{array}\right) D(t)+P(t) D(t)
\end{aligned}
$$

where we denote

$$
P(t)=P(t, r, z)=\left(\begin{array}{lr}
0 & \left(\partial_{z} u^{r}\right)(t, \phi(t, r, z)) \\
\left(\partial_{r} u^{z}\right)(t, \phi(t, r, z)) & 0
\end{array}\right) .
$$

Now since

$$
\frac{\omega^{\theta}(t, \phi(t, r, z))}{\phi^{r}(t, r, z)}=\frac{\omega_{0}^{\theta}(t, r, z)}{r}
$$

we have

$$
\omega^{\theta}(t, r, z)=\frac{\omega_{0}^{\theta}(\tilde{\phi}(t, r, z))}{\tilde{\phi}^{r}(t, r, z)} r .
$$


By (7.3), we have

$$
\begin{aligned}
r & =\phi^{r}\left(t, \tilde{\phi}^{r}(t, r, z), \tilde{\phi}^{z}(t, r, z)\right)-\phi^{r}\left(t, 0, \tilde{\phi}^{z}(t, r, z)\right) \\
& \leq\left\|\partial_{r} \phi^{r}(t, \cdot)\right\|_{\infty} \tilde{\phi}^{r}(t, r, z) \\
& \leq B \tilde{\phi}^{r}(t, r, z) .
\end{aligned}
$$

Therefore

$$
\max _{0 \leq t \leq \frac{1}{B}}\|\omega(t, \cdot)\|_{\infty} \leq \frac{\sqrt{\log A}}{A} B .
$$

Since

$$
\begin{aligned}
& \omega^{\theta}=\partial_{r} u^{z}-\partial_{z} u^{r}, \\
& \frac{1}{r} \partial_{r}\left(r u^{r}\right)+\partial_{z} u^{z}=0,
\end{aligned}
$$

it is not difficult to check that

$$
u^{r}=-\left(\Delta-\frac{1}{r^{2}}\right)^{-1} \partial_{z} \omega^{\theta},
$$

and

$$
\partial_{z} u^{r}=-\partial_{z z}\left(\Delta-\frac{1}{r^{2}}\right)^{-1} \omega^{\theta} .
$$

By (7.26) and (7.31), we get

$$
\left\|\left(\partial_{z} u^{r}\right)(t)\right\|_{\infty} \lesssim B^{2} \frac{\sqrt{\log A}}{A} .
$$

By (7.34) and (7.33), we also get

$$
\begin{aligned}
\left\|\partial_{r} u^{z}(t)\right\|_{\infty} & \lesssim\left\|\omega^{\theta}(t)\right\|_{\infty}+\left\|\left(\partial_{z} u^{r}\right)(t)\right\|_{\infty} \\
& \lesssim B^{2} \frac{\sqrt{\log A}}{A} .
\end{aligned}
$$

Hence

$$
\|P(t)\|_{\infty} \lesssim B^{2} \frac{\sqrt{\log A}}{A} .
$$

Denote

$$
\lambda(t, r, z)=-\left(\partial_{r} u^{r}\right)(t, \phi(t, r, z)) .
$$

By (7.36) and (7.27), we have

$$
-\lambda(t, 0,0) \gtrsim \sqrt{\log A} \cdot B^{-8}, \quad \forall 0 \leq t \leq \frac{1}{B} .
$$

By (7.35), we can write

$$
\begin{aligned}
\left(\partial_{z} u^{z}\right)(t, \phi(t, r, z)) & =-\left(\partial_{r} u^{r}\right)(t, \phi)-\frac{1}{\phi^{r}} u^{r}(t, \phi) \\
& =\lambda(t, r, z)-\frac{1}{\phi^{r}(t, r, z)} u^{r}(t, \phi(t, r, z)) .
\end{aligned}
$$


Using the above computation and integrating (7.32) in time, we get

$$
\begin{aligned}
D(t)= & \left(\begin{array}{cc}
e^{-\int_{0}^{t} \lambda} & 0 \\
0 & e^{\int_{0}^{t} \lambda-\int_{0}^{t} \frac{1}{\phi^{r}} u^{r}(s, \phi) d s}
\end{array}\right) \\
& +\int_{0}^{t}\left(\begin{array}{cc}
e^{-\int_{\tau}^{t} \lambda} & 0 \\
0 & e^{\int_{\tau}^{t} \lambda-\int_{\tau}^{t} \frac{1}{\phi^{r}} u^{r}(s, \phi) d s}
\end{array}\right) P(\tau) D(\tau) d \tau .
\end{aligned}
$$

By (7.5), we have

$$
\max _{0 \leq t \leq \frac{1}{B}} e^{\left|\int_{0}^{t} \frac{u^{r}(s, \phi)}{\phi^{r}}\right|} \lesssim B
$$

Therefore we get

$$
\frac{1}{B} e^{\left|\int_{0}^{t} \lambda\right|} \lesssim B+\max _{0 \leq \tau \leq t}\left(e^{2\left|\int_{0}^{\tau} \lambda\right|}\right) \cdot B^{10} \cdot \frac{\sqrt{\log A}}{A}
$$

or

$$
e^{\left|\int_{0}^{t} \lambda\right|} \lesssim B^{2}+\max _{0 \leq \tau \leq t}\left(e^{2\left|\int_{0}^{\tau} \lambda\right|}\right) \cdot B^{11} \cdot \frac{\sqrt{\log A}}{A} .
$$

Since $B^{12} \ll A / \sqrt{\log A}$, a standard continuity argument then gives

$$
e^{\left|\int_{0}^{t} \lambda(s, r, z) d s\right|} \lesssim B^{2}, \quad \forall 0 \leq t \leq \frac{1}{B}, r \geq 0, z \in \mathbb{R} .
$$

But this obviously contradicts (7.37).

Lemma 7.10 (Vanishing near $r=0$ ). Let $U=U^{r} e_{r}+U^{z} e_{z}$ be a (possibly timedependent) given smooth axi-symmetric without swirl velocity field such that $\nabla \cdot U=$ 0 and

$$
\max _{0 \leq t \leq 1}\left(\left\|D^{2} U(t)\right\|_{4}+\|D U(t)\|_{\infty}+\|U(t)\|_{\infty}\right) \leq C_{1}<\infty .
$$

Suppose $\omega$ is a smooth solution to the linear system

$$
\left\{\begin{array}{l}
\partial_{t}\left(\frac{\omega}{r}\right)+(U \cdot \nabla)\left(\frac{\omega}{r}\right)=0, \quad x=\left(x_{1}, x_{2}, z\right), r=\sqrt{x_{1}^{2}+x_{2}^{2}}, \\
\left.\omega\right|_{t=0}=\omega_{0} .
\end{array}\right.
$$

Here the initial data $\omega_{0} \in C_{c}^{\infty}\left(\mathbb{R}^{3}\right)$ and has the form

$$
\omega_{0}=\omega_{0}^{\theta} e_{\theta},
$$

where $\omega_{0}^{\theta}=\omega_{0}^{\theta}(r, z)$ is scalar-valued and

$$
e_{\theta}=\frac{1}{r}\left(-x_{2}, x_{1}, 0\right) .
$$

Assume that $\omega_{0}$ vanishes near $r=0$, i.e. there exists a constant $r_{0}>0$ such that

$$
\operatorname{supp}\left(\omega_{0}(r, z)\right) \subset\left\{(r, z): r_{0} \leq r \leq \frac{1}{r_{0}}\right\} .
$$

Then there exists a constant $R_{0}=R_{0}\left(r_{0}, C_{1}\right)>0$ such that

$$
\operatorname{supp}(\omega(t, r, z)) \subset\left\{(r, z): R_{0} \leq r \leq \frac{1}{R_{0}}\right\}, \quad \forall 0 \leq t \leq 1 .
$$


Furthermore we have the estimate

$$
\max _{0 \leq t \leq 1}\|\omega(t)\|_{H^{2}} \leq C_{2}
$$

where the constant $C_{2}$ only depends on $\left(\left\|\omega_{0}\right\|_{H^{2}}, r_{0}, C_{1}\right)$.

Proof of Lemma 7.10. The property (7.40) follows easily from finite-speed propagation of the transport equation. For example by (7.9) (with $u^{r}$ replaced by $U^{r}$ ) and (7.38), we have for some $C_{3}=C_{3}\left(C_{1}\right)>0$

$$
\frac{1}{C_{3}} \leq \phi^{r}(t, r, z) / r \leq C_{3}, \quad \forall 0 \leq t \leq 1 .
$$

This shows that the boundary of the support is supported away from $r=0$. Clearly (7.40) follows.

Denote $g=\frac{\omega}{r}$ and $g_{0}=\frac{\omega_{0}}{r}$. Since $\omega$ is supported away from $r=0$, obviously we have

$$
\left\|g_{0}\right\|_{H^{2}} \lesssim r_{0}\left\|\omega_{0}\right\|_{H^{2}}
$$

Since

$$
\partial_{t} g+(U \cdot \nabla) g=0
$$

a simple $H^{2}$ energy estimate then gives

$$
\begin{aligned}
\partial_{t}\left(\|g\|_{H^{2}}^{2}\right) & \lesssim\left\|D^{2} U\right\|_{4}\|\nabla g\|_{4}\|g\|_{H^{2}}+\|D U\|_{\infty}\|g\|_{H^{2}}^{2} \\
& \lesssim C_{1}\|g\|_{H^{2}}^{2} .
\end{aligned}
$$

Therefore

$$
\max _{0 \leq t \leq 1}\|g(t)\|_{H^{2}} \lesssim C_{1}, r_{0}\left\|\omega_{0}\right\|_{H^{2}}
$$

Since $\omega=r g$ and $\omega$ is supported on $r \sim_{r_{0}, C_{1}} 1$, obviously (7.41) follows.

Proposition 7.11. Suppose $\omega$ is a smooth solution to the axisymmetric (without swirl) Euler equation in the form

$$
\left\{\begin{array}{l}
\partial_{t}\left(\frac{\omega}{r}\right)+(u \cdot \nabla)\left(\frac{\omega}{r}\right)=0, \quad 0<t \leq 1, x=\left(x_{1}, x_{2}, z\right), r=\sqrt{x_{1}^{2}+x_{2}^{2}}, \\
u=-\Delta^{-1} \nabla \times \omega \\
\left.\omega\right|_{t=0}=\omega_{0}
\end{array}\right.
$$

and satisfy the following conditions:

- $\omega_{0} \in C_{c}^{\infty}\left(\mathbb{R}^{3}\right)$ and has the form

$$
\omega_{0}=\omega_{0}^{\theta} e_{\theta},
$$

where $\omega_{0}^{\theta}=\omega_{0}^{\theta}(r, z)$ is scalar-valued and $\omega_{0}$ vanishes near $r=0$, i.e. there exists a constant $r_{0}>0$ such that

$$
\operatorname{supp}\left(\omega_{0}(r, z)\right) \subset\left\{(r, z): r>r_{0}\right\} .
$$

- Let $\phi=\left(\phi^{r}, \phi^{z}\right)$ be the characteristic lines defined in (7.2) and $\tilde{\phi}$ be the inverse. For some $0<t_{0} \leq 1, \tilde{r}_{*} \geq 0, \tilde{z}_{*} \in \mathbb{R}$, we have

$$
\left\|(D \tilde{\phi})\left(t_{0}, \tilde{r}_{*}, \tilde{z}_{*}\right)\right\|_{\infty}=\sup _{r \geq 0, z \in \mathbb{R}}\left\|(D \tilde{\phi})\left(t_{0}, r, z\right)\right\|_{\infty}=: M \gg 1 .
$$


Here in (17.44), $\|\cdot\|_{\infty}$ denotes the matrix max norm defined by $\|A\|_{\infty}=$ $\max \left(\left|a_{i j}\right|\right)\left(A=\left(a_{i j}\right)\right)$. The notation $M \gg 1$ means that $M$ is sufficiently large than an absolute constant.

Then we can find a smooth solution also solving the axisymmetric (without swirl) Euler equation

$$
\left\{\begin{array}{l}
\partial_{t}\left(\frac{\tilde{\omega}}{r}\right)+(\tilde{u} \cdot \nabla)\left(\frac{\tilde{\omega}}{r}\right)=0, \quad 0<t \leq 1, \\
\tilde{u}=-\Delta^{-1} \nabla \times \tilde{\omega}, \\
\left.\tilde{\omega}\right|_{t=0}=\tilde{\omega}_{0}
\end{array}\right.
$$

such that the following hold:

(1) $\tilde{\omega}_{0} \in C_{c}^{\infty}\left(\mathbb{R}^{3}\right)$ and has the form

$$
\tilde{\omega}_{0}=\tilde{\omega}_{0}^{\theta} e_{\theta},
$$

where $\tilde{\omega}_{0}^{\theta}=\tilde{\omega}_{0}^{\theta}(r, z)$. The function $\tilde{\omega}_{0}$ also vanishes near $r=0$, i.e. there exists a constant $\tilde{r}_{0}>0$ such that

$$
\operatorname{supp}\left(\tilde{\omega}_{0}(r, z)\right) \subset\left\{(r, z): r>\tilde{r}_{0}\right\} .
$$

Furthermore

$$
\begin{aligned}
& \left\|\frac{\tilde{\omega}_{0}}{r}\right\|_{L^{1}\left(\mathbb{R}^{3}\right)} \leq 2\left\|\frac{\omega_{0}}{r}\right\|_{L^{1}\left(\mathbb{R}^{3}\right)}, \\
& \left\|\frac{\tilde{\omega}_{0}}{r}\right\|_{L^{\infty}\left(\mathbb{R}^{3}\right)} \leq 2\left\|\frac{\omega_{0}}{r}\right\|_{L^{\infty}\left(\mathbb{R}^{3}\right)}, \\
& \left\|\frac{\tilde{\omega}_{0}}{r}\right\|_{L^{3,1}\left(\mathbb{R}^{3}\right)} \leq 2\left\|\frac{\omega_{0}}{r}\right\|_{L^{3,1}\left(\mathbb{R}^{3}\right)} .
\end{aligned}
$$

(2) $\tilde{\omega}_{0}$ is a small perturbation of $\omega_{0}$ :

$$
\begin{aligned}
& \left\|\tilde{\omega}_{0}\right\|_{L^{1}\left(\mathbb{R}^{3}\right)} \leq 2\left\|\omega_{0}\right\|_{L^{1}\left(\mathbb{R}^{3}\right)}, \\
& \left\|\tilde{\omega}_{0}\right\|_{L^{\infty}\left(\mathbb{R}^{3}\right)} \leq 2\left\|\omega_{0}\right\|_{L^{\infty}\left(\mathbb{R}^{3}\right)}, \\
& \left\|\tilde{\omega}_{0}\right\|_{\dot{H}^{\frac{3}{2}\left(\mathbb{R}^{3}\right)}} \leq\left\|\omega_{0}\right\|_{\dot{H}^{\frac{3}{2}}}+\frac{\tilde{C}}{M^{\frac{1}{6}}} .
\end{aligned}
$$

Here $\tilde{C}>0$ is an absolute constant.

(3) For the same $t_{0}$ as in (7.44), we have

$$
\left\|\tilde{\omega}\left(t_{0}\right)\right\|_{\dot{H}^{\frac{3}{2}}}>M^{\frac{1}{6}}
$$

Proof of Proposition 7.11. We begin with a general derivation. Let $W$ be a smooth solution to the system

$$
\left\{\begin{array}{l}
\partial_{t}\left(\frac{W}{r}\right)+(U \cdot \nabla)\left(\frac{W}{r}\right)=0 \\
U=-\Delta^{-1} \nabla \times W \\
\left.W\right|_{t=0}=W_{0}=f e_{\theta} .
\end{array}\right.
$$

Here $f=f(r, z)$ is scalar-valued. Define the corresponding forward characteristic lines $\Phi=\left(\Phi^{r}, \Phi^{z}\right)$ in the same way as (7.2) and let $\tilde{\Phi}$ be the inverse map. Then we have

$$
W(t, x)=W^{\theta}(t, r, z) e_{\theta},
$$


where $W^{\theta}$ is scalar-valued and

$$
\frac{W^{\theta}(t, \Phi(t, r, z))}{\Phi^{r}(t, r, z)}=\frac{f(r, z)}{r} .
$$

Therefore

$$
W^{\theta}(t, r, z)=\frac{f(\tilde{\Phi}(t, r, z))}{\tilde{\Phi}^{r}(t, r, z)} r
$$

and

$$
W(t, x)=\frac{f(\tilde{\Phi}(t, r, z))}{\tilde{\Phi}^{r}(t, r, z)} r e_{\theta}, \quad x=\left(x_{1}, x_{2}, z\right), r=\sqrt{x_{1}^{2}+x_{2}^{2}} .
$$

Now we discuss two cases:

Case 1: $\left\|\omega\left(t_{0}, \cdot\right)\right\|_{H^{\frac{3}{2}}}>M^{\frac{1}{6}}$. In this case we just set $\tilde{\omega}=\omega$ and no work is needed.

Case 2:

$$
\left\|\omega\left(t_{0}, \cdot\right)\right\|_{\dot{H}^{\frac{3}{2}}} \leq M^{\frac{1}{6}} .
$$

In this case in order not to confuse with some notations later on we shall denote $\tilde{\omega}_{0}$ as $W_{0}$ and $\tilde{\omega}$ as $W$. We take the initial data $W_{0}$ in (7.48) as

$$
W_{0}=\omega_{0}+k^{-\frac{3}{2}} G_{0},
$$

where $\omega_{0}$ is the same as in (7.42). The function $G_{0}$ has the form

$$
G_{0}(x)=g_{0}(r, z) e_{\theta}
$$

where $g_{0}$ is scalar-valued. The detailed form of $g_{0}$ will be specified later in the course of the proof.

We shall take the parameter $k$ sufficiently large. In the rest of this proof, to simplify the presentation, we shall use the notation $X=O\left(k^{\alpha}\right)$ ( $\alpha$ is a real number) if the quantity $X$ obeys the bound $X \leq C_{1} k^{\alpha}$ and the positive constant $C_{1}$ can depend on all other parameters except $k$.

Now we assume $G_{0}$ in (7.53) is a smooth compactly-supported function which obeys the following bounds:

$$
\begin{aligned}
& \left\|G_{0}\right\|_{L^{1}\left(\mathbb{R}^{3}\right)}+\left\|G_{0}\right\|_{L^{\infty}\left(\mathbb{R}^{3}\right)}+\left\|\frac{G_{0}}{r}\right\|_{L^{1}\left(\mathbb{R}^{3}\right)}+\left\|\frac{G_{0}}{r}\right\|_{L^{\infty}\left(\mathbb{R}^{3}\right)}=O(1), \\
& \left\|D G_{0}\right\|_{L^{1}\left(\mathbb{R}^{3}\right)}+\left\|D G_{0}\right\|_{L^{\infty}\left(\mathbb{R}^{3}\right)}=O(k), \\
& \left\|D^{2} G_{0}\right\|_{L^{1}\left(\mathbb{R}^{3}\right)}+\left\|D^{2} G_{0}\right\|_{L^{\infty}\left(\mathbb{R}^{3}\right)}=O\left(k^{2}\right) .
\end{aligned}
$$

By (7.50), (7.52), (7.53) and (7.43), we have

$$
\begin{aligned}
W(t, x)= & \frac{\omega_{0}^{\theta}(\tilde{\Phi}(t, r, z))}{\tilde{\Phi}^{r}(t, r, z)} r e_{\theta}+k^{-\frac{3}{2}} \frac{g_{0}(\tilde{\Phi}(t, r, z))}{\tilde{\Phi}^{r}(t, r, z)} r e_{\theta} \\
= & \frac{\omega_{0}^{\theta}(\tilde{\phi}(t, r, z))}{\tilde{\phi}^{r}(t, r, z)} r e_{\theta}+k^{-\frac{3}{2}} \frac{g_{0}(\tilde{\phi}(t, r, z))}{\tilde{\phi}^{r}(t, r, z)} r e_{\theta} \\
& +E_{1}+E_{2},
\end{aligned}
$$


where $\tilde{\phi}$ is the same as in (7.44) and

$$
\begin{aligned}
& E_{1}=\frac{\omega_{0}^{\theta}(\tilde{\Phi}(t, r, z))}{\tilde{\Phi}^{r}(t, r, z)} r e_{\theta}-\frac{\omega_{0}^{\theta}(\tilde{\phi}(t, r, z))}{\tilde{\phi}^{r}(t, r, z)} r e_{\theta}, \\
& E_{2}=k^{-\frac{3}{2}}\left(\frac{g_{0}(\tilde{\Phi}(t, r, z))}{\tilde{\Phi}^{r}(t, r, z)} r e_{\theta}-\frac{g_{0}(\tilde{\phi}(t, r, z))}{\tilde{\phi}^{r}(t, r, z)} r e_{\theta}\right) .
\end{aligned}
$$

We now show that the terms $E_{1}, E_{2}$ in (7.55) are negligible in the computation of $H^{\frac{3}{2}}$-norm of $W$. More precisely, we shall show for some $\alpha>0$,

$$
\max _{0 \leq t \leq 1}\left\|E_{1}(t)\right\|_{H^{\frac{3}{2}\left(\mathbb{R}^{3}\right)}}+\max _{0 \leq t \leq 1}\left\|E_{2}(t)\right\|_{H^{\frac{3}{2}\left(\mathbb{R}^{3}\right)}}=O\left(k^{-\alpha}\right) .
$$

To show (7.56), let us introduce $\omega_{2}, W_{1}, W_{2}$ which solve the following linear systems:

$$
\begin{aligned}
& \left\{\begin{array}{l}
\partial_{t}\left(\frac{\omega_{2}}{r}\right)+(U \cdot \nabla)\left(\frac{\omega_{2}}{r}\right)=0, \\
\left.\omega_{2}\right|_{t=0}=\omega_{0} ;
\end{array}\right. \\
& \left\{\begin{array}{l}
\partial_{t}\left(\frac{W_{1}}{r}\right)+(u \cdot \nabla)\left(\frac{W_{1}}{r}\right)=0, \\
\left.W_{1}\right|_{t=0}=k^{-\frac{3}{2}} G_{0} ;
\end{array}\right. \\
& \left\{\begin{array}{l}
\partial_{t}\left(\frac{W_{2}}{r}\right)+(U \cdot \nabla)\left(\frac{W_{2}}{r}\right)=0, \\
\left.W_{2}\right|_{t=0}=k^{-\frac{3}{2}} G_{0} ;
\end{array}\right.
\end{aligned}
$$

Here the drift terms $u, U$ and the data $\omega_{0}, G_{0}$ are the same as in the nonlinear systems (7.42) and (7.48).

It is not difficult to check that

$$
\begin{aligned}
& W=\omega+W_{1}+E_{1}+E_{2}, \\
& E_{1}=\omega_{2}-\omega, \\
& E_{2}=W_{2}-W_{1} .
\end{aligned}
$$

Therefore we only need to run perturbation arguments between the nonlinear systems (7.42), (7.48) and the linear systems (7.57)-(7.59).

We first control the drift difference $u-U$.

By (7.52) and (7.54), we have $\left\|W_{0}\right\|_{W^{1, p}}=O(1)$ for any $1 \leq p \leq \infty$.

Thanks to the axisymmetry without swirl, we may write the system (7.48) as either

$$
\partial_{t} W+(U \cdot \nabla) W=(W \cdot \nabla) U
$$

or

$$
\partial_{t} W+(U \cdot \nabla) W=\frac{U^{r}}{r} W .
$$

Take any $3<p<\infty$. A standard energy estimate on (7.60) in $W^{1, p}$ gives

$$
\frac{d}{d t}\left(\|W(t)\|_{W^{1, p}}^{p}\right) \lesssim\left(\|D u(t)\|_{\infty}+\|W(t)\|_{\infty}\right)\|W(t)\|_{W^{1, p}}^{p} .
$$

Note that by (7.54) and Lemma 7.2 ,

$$
\max _{0 \leq t \leq 1}\left\|\frac{U^{r}(t)}{r}\right\|_{\infty} \lesssim\left\|\frac{W_{0}}{r}\right\|_{L^{3,1}}=O(1) .
$$


Therefore by (7.61), we have

$$
\max _{0 \leq t \leq 1}\left(\|W(t)\|_{2}+\|W(t)\|_{\infty}\right)=O(1)
$$

By the usual log-interpolation inequality, we have

$$
\begin{aligned}
\|D U(t)\|_{\infty} & \lesssim\|W(t)\|_{2}+\log \left(10+\|W(t)\|_{W^{1, p}}^{p}\right)\|W(t)\|_{\infty} \\
& \lesssim O(1) \cdot \log \left(10+\|W(t)\|_{W^{1, p}}^{p}\right) .
\end{aligned}
$$

Plugging the last estimate into (7.62), we obtain

$$
\frac{d}{d t}\left(\|W(t)\|_{W^{1, p}}^{p}\right) \lesssim O(1) \cdot \log \left(10+\|W(t)\|_{W^{1, p}}^{p}\right)\|W(t)\|_{W^{1, p}}^{p} .
$$

Integrating in time then gives

$$
\max _{0 \leq t \leq 1}\|W(t)\|_{W^{1, p}}=O(1), \quad \forall 3<p<\infty .
$$

By Sobolev embedding, we get

$$
\max _{0 \leq t \leq 1}\left(\left\|D^{2} U(t)\right\|_{p}+\|D U(t)\|_{\infty}\right)=O(1), \quad \forall 3<p<\infty .
$$

Similarly using (7.54) we can derive

$$
\max _{0 \leq t \leq 1}\|W(t)\|_{H^{2}}=O\left(k^{\frac{1}{2}}\right) .
$$

Note that the system (7.42) is independent of the parameter $k$, therefore we have

$$
\max _{0 \leq t \leq 1}\|u(t)\|_{W^{20, p}}=O(1), \quad \forall 2 \leq p<\infty .
$$

Now to control the difference, we recall

$$
\left\{\begin{array}{l}
\partial_{t} \omega+(u \cdot \nabla) \omega=(\omega \cdot \nabla) u, \\
\partial_{t} W+(U \cdot \nabla) W=(W \cdot \nabla) U \\
\left.(W-\omega)\right|_{t=0}=k^{-\frac{3}{2}} G_{0} .
\end{array}\right.
$$

Obviously

$$
\begin{gathered}
\partial_{t}(W-\omega)+(U \cdot \nabla)(W-\omega)+((U-u) \cdot \nabla) \omega \\
=(W \cdot \nabla)(U-u)+((W-\omega) \cdot \nabla) u .
\end{gathered}
$$

By (7.63), (7.64), (7.66) and Sobolev embedding, we then obtain

$$
\begin{aligned}
\partial_{t}\left(\|W-\omega\|_{2}^{2}\right) \lesssim & \|U-u\|_{6} \cdot\|W-\omega\|_{2} \cdot\|\nabla \omega\|_{3} \\
& \quad+\|W\|_{\infty} \cdot\|D(U-u)\|_{2} \cdot\|W-\omega\|_{2}+\|D u\|_{\infty} \cdot\|W-\omega\|_{2}^{2} \\
& \lesssim O(1) \cdot\|W-\omega\|_{2}^{2} .
\end{aligned}
$$

Therefore

$$
\max _{0 \leq t \leq 1}\|W(t)-\omega(t)\|_{2}=O\left(k^{-\frac{3}{2}}\right) .
$$

In a similar way, we can derive

$$
\begin{aligned}
& \max _{0 \leq t \leq 1}\|W(t)-\omega(t)\|_{p}=O\left(k^{-\frac{3}{2}}\right), \quad \forall 1<p<\infty, \\
& \max _{0 \leq t \leq 1}\left(\|U(t)-u(t)\|_{p}+\|\nabla(U(t)-u(t))\|_{p}\right)=O\left(k^{-\frac{3}{2}}\right), \quad \forall 2 \leq p<\infty .
\end{aligned}
$$


We are now ready to control $E_{1}=\omega_{2}-\omega$. By (7.57), (7.64) and Lemma 7.10, we have

$$
\max _{0 \leq t \leq 1}\left\|\omega_{2}(t)\right\|_{H^{2}}=O(1)
$$

By (7.66), we get

$$
\max _{0 \leq t \leq 1}\left\|\omega_{2}(t)-\omega(t)\right\|_{H^{2}}=O(1) .
$$

On the other hand, using (7.67), it is not difficult to check that

$$
\max _{0 \leq t \leq 1}\left\|\omega_{2}(t)-\omega(t)\right\|_{2}=O\left(k^{-\frac{3}{2}}\right)
$$

Interpolating the above two bounds then gives

$$
\max _{0 \leq t \leq 1}\left\|\omega_{2}(t)-\omega(t)\right\|_{H^{\frac{3}{2}}}=O\left(k^{-\frac{3}{8}}\right) .
$$

Therefore $E_{1}$ is OK for us.

To control $E_{2}$, we note that by (7.58) $-(7.59)$, we have

$$
\begin{aligned}
& \partial_{t} W_{1}+(u \cdot \nabla) W_{1}=\left(W_{1} \cdot \nabla\right) u \\
& \partial_{t} W_{2}+(U \cdot \nabla) W_{2}=\left(W_{2} \cdot \nabla\right) U \\
& \partial_{t}\left(W_{1}-W_{2}\right)+((u-U) \cdot \nabla) W_{1}+(U \cdot \nabla)\left(W_{1}-W_{2}\right) \\
& \quad=\left(\left(W_{1}-W_{2}\right) \cdot \nabla\right) u+\left(W_{2} \cdot \nabla\right)(u-U) .
\end{aligned}
$$

For (7.68), a simple energy estimate using (7.54) and (7.66) gives

$$
\begin{aligned}
& \max _{0 \leq t \leq 1}\left\|W_{1}\right\|_{2}=O\left(k^{-\frac{3}{2}}\right), \\
& \max _{0 \leq t \leq 1}\left\|\nabla W_{1}\right\|_{4}=O\left(k^{-\frac{1}{2}}\right), \\
& \max _{0 \leq t \leq 1}\left\|W_{1}\right\|_{H^{2}}=O\left(k^{\frac{1}{2}}\right) .
\end{aligned}
$$

Similarly for (7.69), we use (7.54), (7.64) and (7.65) to get

$$
\begin{aligned}
& \max _{0 \leq t \leq 1}\left\|W_{2}\right\|_{4}=O\left(k^{-\frac{3}{2}}\right), \\
& \max _{0 \leq t \leq 1}\left\|W_{2}\right\|_{H^{2}}=O\left(k^{\frac{1}{2}}\right) .
\end{aligned}
$$

For (7.70), a simple $L^{2}$ estimate using (7.67), (7.71) and (7.72) gives

$$
\begin{aligned}
\partial_{t}\left(\left\|W_{1}-W_{2}\right\|_{2}^{2}\right) \lesssim & \left\|W_{1}-W_{2}\right\|_{2} \cdot\|u-U\|_{4} \cdot\left\|\nabla W_{1}\right\|_{4} \\
& +\left\|W_{1}-W_{2}\right\|_{2}^{2} \cdot\|\nabla u\|_{\infty}+\left\|W_{2}\right\|_{4} \cdot\|\nabla(u-U)\|_{4} \cdot\left\|W_{1}-W_{2}\right\|_{2} \\
\lesssim & O\left(k^{-2}\right) \cdot\left\|W_{1}-W_{2}\right\|_{2}+O(1) \cdot\left\|W_{1}-W_{2}\right\|_{2}^{2} \\
& \quad+O\left(k^{-3}\right) \cdot\left\|W_{1}-W_{2}\right\|_{2} .
\end{aligned}
$$

Gronwall in time then gives

$$
\max _{0 \leq t \leq 1}\left\|W_{1}(t)-W_{2}(t)\right\|_{2}=O\left(k^{-2}\right) .
$$

Interpolating this with the trivial estimate

$$
\max _{0 \leq t \leq 1}\left\|W_{1}(t)-W_{2}(t)\right\|_{H^{2}}=O\left(k^{\frac{1}{2}}\right)
$$


then yields

$$
\max _{0 \leq t \leq 1}\left\|W_{1}(t)-W_{2}(t)\right\|_{H^{\frac{3}{2}}}=O\left(k^{-\frac{1}{8}}\right) .
$$

This shows that $\left\|E_{2}\right\|_{H^{\frac{3}{2}}}=O\left(k^{-\frac{1}{8}}\right)$ and we have finished the proof of (7.56).

We now specify the choice of $g_{0}$ in (7.53).

By (7.44), we have

$$
\begin{aligned}
& \max \left\{\left|\left(\partial_{r} \tilde{\phi}^{r}\right)\left(t_{0}, \tilde{r}_{*}, \tilde{z}_{*}\right)\right|, \quad\left|\left(\partial_{z} \tilde{\phi}^{r}\right)\left(t_{0}, \tilde{r}_{*}, \tilde{z}_{*}\right)\right|,\right. \\
& \left.\quad\left|\left(\partial_{r} \tilde{\phi}^{z}\right)\left(t_{0}, \tilde{r}_{*}, \tilde{z}_{*}\right)\right|, \quad\left|\left(\partial_{z} \tilde{\phi}^{z}\right)\left(t_{0}, \tilde{r}_{*}, \tilde{z}_{*}\right)\right|\right\}=M .
\end{aligned}
$$

WLOG we assume

$$
\left|\left(\partial_{r} \tilde{\phi}^{r}\right)\left(t_{0}, \tilde{r}_{*}, \tilde{z}_{*}\right)\right|=M
$$

The other cases are similarly treated.

Let $\left(r_{*}, z_{*}\right)$ be the pre-image of $\left(\tilde{r}_{*}, \tilde{z}_{*}\right)$, i.e. $\tilde{r}_{*}=\phi^{r}\left(t_{0}, r_{*}, z_{*}\right), \tilde{z}_{*}=\phi^{z}\left(t_{0}, r_{*}, z_{*}\right)$. By (7.7), we have

$$
\left|\operatorname{det}\left((D \tilde{\phi})\left(t_{0}, \phi\left(t_{0}, r_{*}, z_{*}\right)\right)\right)\right|=\frac{\phi^{r}\left(t_{0}, r_{*}, z_{*}\right)}{r_{*}}=: N_{*}>0 .
$$

By the Fundamental Theorem of Calculus and (7.3), we have

$$
\begin{aligned}
r_{*} & =\tilde{\phi}^{r}\left(t_{0}, \phi^{r}\left(t_{0}, r_{*}, z_{*}\right), \phi^{z}\left(t_{0}, r_{*}, z_{*}\right)\right)-\tilde{\phi}^{r}\left(t_{0}, 0, \phi^{z}\left(t_{0}, r_{*}, z_{*}\right)\right) \\
& \leq\left\|\partial_{r} \tilde{\phi}^{r}\right\|_{\infty} \cdot \phi^{r}\left(t_{0}, r_{*}, z_{*}\right) \\
& \leq M \cdot \phi^{r}\left(t_{0}, r_{*}, z_{*}\right) .
\end{aligned}
$$

Therefore

$$
N_{*} M \geq 1
$$

This relation will be used later.

By (7.73), (7.74) and continuity, we can find a nonempty open set $\Omega_{0}$ around the point $\left(r_{*}, z_{*}\right)$ such that

$$
\begin{aligned}
& \frac{M}{2}<\left|\left(\partial_{r} \tilde{\phi}^{r}\right)\left(t_{0}, \phi\left(t_{0}, r, z\right)\right)\right|<2 M, \\
& \frac{N_{*}}{2}<\frac{\phi^{r}\left(t_{0}, r, z\right)}{r}=\left|\operatorname{det}\left((D \tilde{\phi})\left(t_{0}, \phi\left(t_{0}, r, z\right)\right)\right)\right|<2 N_{*}, \quad \forall(r, z) \in \Omega_{0} .
\end{aligned}
$$

Furthermore we may shrink $\Omega_{0}$ slightly if necessary such that for some $\delta_{1}>0$,

$$
\Omega_{0} \cap\left\{(r, z): 0 \leq r \leq \delta_{1}\right\}=\emptyset
$$

In yet other words, if $(r, z) \in \Omega_{0}$, then we must have $r>\delta_{1}$.

Now choose $b \in C_{c}^{\infty}\left(\Omega_{0}\right)$ such that

$$
\int|b(r, z)|^{2} r d r d z=1
$$

Since by our choice $\Omega_{0}$ stays away from the axis $r=0$, the function $b$ can be naturally regarded as a smooth function on $\mathbb{R}^{3}$.

We now let

$$
g_{0}(r, z)=\frac{1}{M^{\frac{1}{6}}} \cos (k r) b(r, z)
$$


and recall from (7.53)

$$
\begin{aligned}
G_{0}(x) & =g_{0}(r, z) e_{\theta} \\
& =\frac{1}{M^{\frac{1}{6}}} \cos (k r) b(r, z) e_{\theta} .
\end{aligned}
$$

By (7.77), it is not difficult to check that (7.54) is satisfied.

Since

$$
W_{0}=\omega_{0}+k^{-\frac{3}{2}} G_{0},
$$

by taking $k$ sufficiently large, obviously we can have

$$
\begin{aligned}
& \left\|W_{0}\right\|_{L^{1}\left(\mathbb{R}^{3}\right)} \leq 2\left\|\omega_{0}\right\|_{L^{1}\left(\mathbb{R}^{3}\right)}, \\
& \left\|W_{0}\right\|_{L^{\infty}\left(\mathbb{R}^{3}\right)} \leq 2\left\|\omega_{0}\right\|_{L^{\infty}\left(\mathbb{R}^{3}\right)}, \\
& \left\|\frac{W_{0}}{r}\right\|_{L^{1}\left(\mathbb{R}^{3}\right)} \leq 2\left\|\frac{\omega_{0}}{r}\right\|_{L^{1}\left(\mathbb{R}^{3}\right)}, \\
& \left\|\frac{W_{0}}{r}\right\|_{L^{\infty}\left(\mathbb{R}^{3}\right)} \leq 2\left\|\frac{\omega_{0}}{r}\right\|_{L^{\infty}\left(\mathbb{R}^{3}\right)}, \\
& \left\|\frac{W_{0}}{r}\right\|_{L^{3,1}\left(\mathbb{R}^{3}\right)} \leq 2\left\|\frac{\omega_{0}}{r}\right\|_{L^{3,1}\left(\mathbb{R}^{3}\right)} .
\end{aligned}
$$

Therefore (7.45) and the first two conditions in (7.46) are easily satisfied. To check the third condition therein, we note that by (7.77) and for $k$ sufficiently large,

$$
\begin{aligned}
\left\|G_{0}\right\|_{L^{2}\left(\mathbb{R}^{3}\right)} & \lesssim \frac{1}{M^{\frac{1}{6}}}, \\
\left\|G_{0}\right\|_{H^{2}\left(\mathbb{R}^{3}\right)} & \lesssim \frac{1}{M^{\frac{1}{6}}} \cdot k^{2} .
\end{aligned}
$$

Here the implied constants are absolute constants. Interpolation then gives

$$
\left\|G_{0}\right\|_{H^{\frac{3}{2}\left(\mathbb{R}^{3}\right)}} \lesssim \frac{1}{M^{\frac{1}{6}}} k^{\frac{3}{2}} .
$$

Thus all conditions in (7.45) and (7.46) are satisfied.

It remains to show (7.47).

By (7.51), (7.55) and (7.56), we have

$$
\begin{aligned}
\left\|W\left(t_{0}, \cdot\right)\right\|_{\dot{H}^{\frac{3}{2}}} \geq\left\|k^{-\frac{3}{2}} \cdot \frac{g_{0}\left(\tilde{\phi}\left(t_{0}\right)\right)}{\tilde{\phi}^{r}\left(t_{0}\right)} r e_{\theta}\right\|_{\dot{H}^{\frac{3}{2}}} \\
\quad-\left\|\frac{\omega_{0}^{\theta}\left(\tilde{\phi}\left(t_{0}\right)\right)}{\tilde{\phi}^{r}\left(t_{0}\right)} r e_{\theta}\right\|_{\dot{H}^{\frac{3}{2}}} \\
\quad-\left\|E_{1}\right\|_{\dot{H}^{\frac{3}{2}}}-\left\|E_{2}\right\|_{\dot{H}^{\frac{3}{2}}} \\
\geq\left\|k^{-\frac{3}{2}} \cdot \frac{g_{0}\left(\tilde{\phi}\left(t_{0}\right)\right)}{\tilde{\phi}^{r}\left(t_{0}\right)} r e_{\theta}\right\|_{\dot{H}^{\frac{3}{2}}}-M^{\frac{1}{6}}-O\left(k^{-\alpha}\right) .
\end{aligned}
$$

Therefore (7.47) will be established once we prove the stronger estimate

$$
\left\|k^{-\frac{3}{2}} \cdot \frac{g_{0}\left(\tilde{\phi}\left(t_{0}\right)\right)}{\tilde{\phi}^{r}\left(t_{0}\right)} r e_{\theta}\right\|_{\dot{H}^{\frac{3}{2}}} \gtrsim M^{\frac{1}{3}} .
$$

We shall prove this via interpolation and inflation of $H^{1}$ norm. 
By (7.78), we have

$$
\begin{aligned}
\left\|k^{-\frac{3}{2}} \cdot \frac{g_{0}\left(\tilde{\phi}\left(t_{0}\right)\right)}{\tilde{\phi}^{r}\left(t_{0}\right)} r e_{\theta}\right\|_{L^{2}\left(\mathbb{R}^{3}\right)} & \lesssim k^{-\frac{3}{2}}\left(\int\left|\frac{g_{0}\left(\tilde{\phi}\left(t_{0}\right)\right)}{\tilde{\phi}^{r}\left(t_{0}\right)} r\right|^{2} r d r d z\right)^{\frac{1}{2}} \\
& \lesssim k^{-\frac{3}{2}}\left(\int\left|\frac{g_{0}(r, z) \phi^{r}\left(t_{0}, r, z\right)}{r}\right|^{2} r d r d z\right)^{\frac{1}{2}} \\
& \lesssim \frac{k^{-\frac{3}{2}}}{M^{\frac{1}{6}}}\left(\int \frac{\cos ^{2}(k r) b^{2}(r, z)\left(\phi^{r}\left(t_{0}, r, z\right)\right)^{2}}{r^{2}} r d r d z\right)^{\frac{1}{2}} \\
& \lesssim \frac{k^{-\frac{3}{2}}}{M^{\frac{1}{6}}}\left\|\frac{b \phi^{r}\left(t_{0}\right)}{r}\right\|_{L^{2}\left(\mathbb{R}^{3}\right)} \lesssim \frac{k^{-\frac{3}{2}}}{M^{\frac{1}{6}}} N_{*},
\end{aligned}
$$

where in the last inequality we have used (7.76) and (7.77).

Now introduce

$$
g_{1}(r, z)=\sin \left(k \tilde{\phi}^{r}\left(t_{0}, r, z\right)\right) \frac{b\left(\tilde{\phi}\left(t_{0}, r, z\right)\right)}{\tilde{\phi}^{r}\left(t_{0}, r, z\right)}\left(\partial_{r} \tilde{\phi}^{r}\right)\left(t_{0}, r, z\right) r e_{\theta} .
$$

By (7.76) and a similar calculation as in (7.80), we have for $k$ sufficiently large,

$$
\begin{aligned}
\left\|g_{1}\right\|_{L^{2}(r d r d z)} & \geq\left(\int \frac{\sin ^{2}(k r) b^{2}(r, z)\left(\left(\partial_{r} \tilde{\phi}^{r}\right)\left(t_{0}, \phi\left(t_{0}, r, z\right)\right)\right)^{2}}{r^{2}}\left(\phi^{r}\left(t_{0}, r, z\right)\right)^{2} r d r d z\right)^{\frac{1}{2}} \\
& \geq M\left\|\frac{b \phi^{r}\left(t_{0}\right)}{r}\right\|_{L^{2}\left(\mathbb{R}^{3}\right)}-O\left(k^{-\alpha}\right) \\
& \geq \frac{2}{3} M\left\|\frac{b \phi^{r}\left(t_{0}\right)}{r}\right\|_{L^{2}\left(\mathbb{R}^{3}\right)} \gtrsim M \cdot N_{*} .
\end{aligned}
$$

Now for the $\dot{H}^{1}$-norm, by using (7.81), we have

$$
\begin{aligned}
\left\|k^{-\frac{3}{2}} \cdot \frac{g_{0}\left(\tilde{\phi}\left(t_{0}\right)\right)}{\tilde{\phi}^{r}\left(t_{0}\right)} r e_{\theta}\right\|_{\dot{H}^{1}\left(\mathbb{R}^{3}\right)} & \geq k^{-\frac{3}{2}}\left\|\partial_{r}\left(\frac{g_{0}\left(\tilde{\phi}\left(t_{0}\right)\right)}{\tilde{\phi}^{r}\left(t_{0}\right)} r e_{\theta}\right)\right\|_{L^{2}(r d r d z)} \\
& \geq \frac{k^{-\frac{3}{2}}}{M^{\frac{1}{6}}} \cdot\left(k\left\|g_{1}\right\|_{L^{2}(r d r d z)}+O(1)\right) \\
& \geq \frac{1}{2} k^{-\frac{1}{2}} M^{\frac{5}{6}} N_{*},
\end{aligned}
$$

where again we need to take $k$ sufficiently large.

We are now ready to prove (7.79).

By the usual interpolation inequality

$$
\|f\|_{\dot{H}^{1}\left(\mathbb{R}^{3}\right)} \lesssim\|f\|_{L^{2}\left(\mathbb{R}^{3}\right)}^{\frac{1}{3}} \cdot\|f\|_{\dot{H}^{\frac{3}{2}}\left(\mathbb{R}^{3}\right)}^{\frac{2}{3}}
$$

and (7.80), (7.82), we have

$$
\begin{aligned}
& k^{-\frac{1}{2}} M^{\frac{5}{6}} N_{*} \\
& \quad \lesssim\left(\frac{k^{-\frac{3}{2}} N_{*}}{M^{\frac{1}{6}}}\right)^{\frac{1}{3}} \cdot\left\|k^{-\frac{3}{2}} \cdot \frac{g_{0}\left(\tilde{\phi}\left(t_{0}\right)\right)}{\tilde{\phi}^{r}\left(t_{0}\right)} r e_{\theta}\right\|_{\dot{H}^{\frac{3}{2}}}^{\frac{2}{3}}\left(\mathbb{R}^{3}\right)
\end{aligned}
$$


By (7.75), we then have

$$
\begin{aligned}
\left\|k^{-\frac{3}{2}} \cdot \frac{g_{0}\left(\tilde{\phi}\left(t_{0}\right)\right)}{\tilde{\phi}^{r}\left(t_{0}\right)} r e_{\theta}\right\|_{\dot{H}^{\frac{3}{2}}\left(\mathbb{R}^{3}\right)}^{\frac{2}{3}} & \gtrsim M^{\frac{8}{9}} N_{*}^{\frac{2}{3}} \\
& \gtrsim M^{\frac{2}{9}} .
\end{aligned}
$$

Hence

$$
\left\|k^{-\frac{3}{2}} \cdot \frac{g_{0}\left(\tilde{\phi}\left(t_{0}\right)\right)}{\tilde{\phi}^{r}\left(t_{0}\right)} r e_{\theta}\right\|_{\dot{H}^{\frac{3}{2}}\left(\mathbb{R}^{3}\right)} \gtrsim M^{\frac{1}{3}} \gg M^{\frac{1}{6}} .
$$

This ends the estimate of (7.79).

Proposition 7.12. For any $A \gg 1$, there exist $\delta_{0}=\delta_{0}(A) \rightarrow 0, t_{0}=t_{0}(A) \rightarrow 0$, $M_{0}=M_{0}(A) \rightarrow \infty$ (as $\left.A \rightarrow \infty\right)$, and a smooth solution $\omega$ to the axisymmetric (without swirl) Euler equation

$$
\left\{\begin{array}{l}
\partial_{t}\left(\frac{\omega}{r}\right)+(u \cdot \nabla)\left(\frac{\omega}{r}\right)=0, \quad 0<t \leq 1, x=\left(x_{1}, x_{2}, z\right), r=\sqrt{x_{1}^{2}+x_{2}^{2}}, \\
u=-\Delta^{-1} \nabla \times \omega \\
\left.\omega\right|_{t=0}=\omega_{0}
\end{array}\right.
$$

such that the following conditions are satisfied:

(1) $\omega_{0} \in C_{c}^{\infty}\left(\mathbb{R}^{3}\right), \omega_{0}=\omega_{0}^{\theta}(r, z) e_{\theta}$ and for some $r_{0}>0$,

$$
\operatorname{supp}\left(\omega_{0}^{\theta}(r, z)\right) \subset\left\{(r, z): r>r_{0}\right\} .
$$

(2) The $L^{\infty}$ norm of $\omega$ is uniformly small on the interval $[0,1]$ :

$$
\max _{0 \leq t \leq 1}\|\omega(t)\|_{L^{\infty}} \leq \delta_{0}(A) .
$$

(3) The support of $\omega(t)$ remains close to the origin:

$$
\operatorname{supp}(\omega(t, \cdot)) \subset\left\{x: \quad|x|<\delta_{0}(A)\right\}, \quad \forall 0 \leq t \leq 1 .
$$

(4) The $\dot{H}^{\frac{3}{2}}$-norm of $\omega$ is inflated rapidly from $t=0$ to $t=t_{0}$ :

$$
\begin{aligned}
& \left\|\omega_{0}\right\|_{\dot{H}^{\frac{3}{2}}}<\delta_{0}(A), \\
& \left\|\omega\left(t_{0}\right)\right\|_{\dot{H}^{\frac{3}{2}}}>M_{0}(A) .
\end{aligned}
$$

Proof of Proposition 7.12. We first note that it suffices to construct the solution $\omega$ satisfying all other conditions except (7.85). Indeed if $\omega$ is such a solution, then for any $\lambda>0$,

$$
\omega_{\lambda}(t, x):=\omega(t, \lambda x)
$$

is also a solution to the Euler equation. By finite speed propagation, we have

$$
\operatorname{supp}(\omega(t)) \subset K, \quad \forall 0 \leq t \leq 1,
$$

where $K$ is a fixed compact set. On the other hand

$$
\operatorname{supp}\left(\omega_{\lambda}(t)\right) \subset \frac{1}{\lambda} K=\left\{\frac{1}{\lambda} x: x \in K\right\}, \quad \forall 0 \leq t \leq 1 .
$$

Obviously by taking $\lambda$ sufficiently large we can satisfy (7.85). Note that (77.84), (7.86) is invariant under the scaling transformation $x \rightarrow \lambda x$. Therefore in the rest of this proof we shall ignore (7.85). 
For $A \gg 1$, we choose $g_{A}$ as in (7.11) and denote by $W$ the corresponding smooth solution to the Euler equation:

$$
\left\{\begin{array}{l}
\partial_{t}\left(\frac{W}{r}\right)+(U \cdot \nabla)\left(\frac{W}{r}\right)=0, \quad-2 \leq t \leq 2 \\
\nabla \cdot U=0 \\
\left.W\right|_{t=0}=W_{0}=g_{A} e_{\theta} .
\end{array}\right.
$$

By (7.16) we have (recall $U=U^{r} e_{r}+U^{z} e_{z}$ )

$$
\begin{aligned}
\left\|\frac{U^{r}(t)}{r}\right\|_{\infty} & \leq C\left\|\frac{W(t)}{r}\right\|_{L^{3,1}} \\
& \leq C \sqrt{\log A}, \quad \forall t \in \mathbb{R},
\end{aligned}
$$

where $C>0$ is an absolute constant and we have used the $L^{3,1}$-preservation of $W / r$ :

$$
\left\|\frac{W(t)}{r}\right\|_{L^{3,1}}=\left\|\frac{W_{0}}{r}\right\|_{L^{3,1}}, \quad \forall t \in \mathbb{R} .
$$

Since

$$
\partial_{t} W+(U \cdot \nabla) W=\frac{U^{r}}{r} W
$$

we get

$$
\begin{aligned}
\max _{-2 \leq t \leq 2}\|W(t)\|_{\infty} & \leq\left\|W_{0}\right\|_{\infty} e^{\max -2 \leq t \leq 2}\left\|\frac{U^{r}}{r}\right\|_{\infty} \\
& \leq \frac{\sqrt{\log A}}{A} e^{C \sqrt{\log A}}<A^{-\frac{1}{2}}
\end{aligned}
$$

for $A$ sufficiently large.

By definition of $g_{A}$, the condition (7.83) is trivially satisfied. It remains to check (7.86). By (7.17), we have

$$
\left\|W_{0}\right\|_{\dot{H}^{\frac{3}{2}}} \lesssim \frac{\sqrt{\log A}}{\sqrt{A}} .
$$

Let $\Phi=\left(\Phi^{r}, \Phi^{z}\right)$ be the forward characteristic lines as in (7.2) and let $\tilde{\Phi}$ be the inverse. By Proposition 7.9 we have for some $0<t_{1} \leq \frac{1}{\log \log A}$,

$$
\left\|D \Phi\left(t_{1}\right)\right\|_{\infty}+\left\|D \tilde{\Phi}\left(t_{1}\right)\right\|_{\infty} \geq \log \log A .
$$

By differentiating the identity $\Phi \circ \tilde{\Phi}=i d$ and using (7.7), we have

$$
\begin{aligned}
(D \Phi)(\tilde{\Phi}(r, z)) & =((D \tilde{\Phi})(r, z))^{-1} \\
& =\frac{1}{\operatorname{det}(D \tilde{\Phi}(r, z))} \operatorname{adj}((D \tilde{\Phi})(r, z)) \\
& =\frac{\tilde{\Phi}^{r}(r, z)}{r} \operatorname{adj}((D \tilde{\Phi})(r, z)),
\end{aligned}
$$

where $\operatorname{adj}((D \tilde{\Phi})(r, z))$ is the adjugate matrix of $D \tilde{\phi}(r, z)$. Recall that for any $2 \times 2$ matrix

$$
B=\left(\begin{array}{ll}
a & b \\
c & d
\end{array}\right)
$$


we have

$$
\operatorname{adj}(B)=\left(\begin{array}{cc}
d & -b \\
-c & a
\end{array}\right)
$$

and obviously

$$
\begin{aligned}
\|B\|_{\infty} & =\max \{|a|,|b|,|c|,|d|\} \\
& =\|\operatorname{adj}(B)\|_{\infty} .
\end{aligned}
$$

Therefore

$$
\begin{aligned}
\left\|D \Phi\left(t_{1}\right)\right\|_{\infty} & \leq\left\|\frac{\tilde{\Phi}^{r}}{r}\right\|_{\infty}\|\operatorname{adj}(D \tilde{\Phi})\|_{\infty} \\
& \leq\left\|D \tilde{\Phi}\left(t_{1}\right)\right\|_{\infty}^{2}
\end{aligned}
$$

Consequently we have

$$
\left\|D \tilde{\Phi}\left(t_{1}\right)\right\|_{\infty} \gtrsim \sqrt{\log \log A} .
$$

We can then apply Proposition 7.11 (with $W$ as the input solution $\omega$ ) and obtain $\tilde{\omega}$ as the desired solution (note that $\left\|\frac{\tilde{\omega}_{0}}{r}\right\|_{L^{3,1}} \lesssim \sqrt{\log A},\left\|\tilde{\omega}_{0}\right\|_{\infty} \lesssim \frac{\sqrt{\log A}}{A}$ so that we can repeat the computation of (7.87) and still have $\max _{0 \leq t \leq 1}\|\tilde{\omega}(t)\|_{\infty} \lesssim A^{-\frac{1}{2}}$.)

Lemma 7.13. Suppose $\omega^{1}, \omega^{2}$ are given smooth solutions to the $3 D$ Euler equations (in vorticity form):

$$
\left\{\begin{array}{l}
\partial_{t} \omega^{j}+\left(u^{j} \cdot \nabla\right) \omega^{j}=\left(\omega^{j} \cdot \nabla\right) u^{j}, \quad 0<t \leq 1 \\
u^{j}=-\Delta^{-1} \nabla \times \omega^{j} \\
\left.\omega^{j}\right|_{t=0}=\omega_{0}^{j} \in C_{c}^{\infty}\left(\mathbb{R}^{3}\right), \quad j=1,2 .
\end{array}\right.
$$

Here we assume the lifespan of each $\omega^{j}$ is at least $[0,1]$.

Define

$$
r_{0}=\max _{j=1,2} \max _{0 \leq t \leq 1}\left\|u^{j}(t)\right\|_{\infty}
$$

Consider the problem

$$
\left\{\begin{array}{l}
\partial_{t} W+(U \cdot \nabla) W=(W \cdot \nabla) U \\
U=-\Delta^{-1} \nabla \times W \\
\left.W\right|_{t=0}=W_{0}
\end{array}\right.
$$

where

$$
W_{0}(x)=\omega_{0}^{1}(x)+\omega_{0}^{2}\left(x-x_{W}\right)
$$

and $x_{W} \in \mathbb{R}^{3}$ is a vector which controls the mutual distance between $\omega_{0}^{1}$ and $\omega_{0}^{2}$.

For any $\epsilon>0$, there exists $R_{\epsilon}=R_{\epsilon}\left(\epsilon, \max _{j=1,2} \max _{0 \leq t \leq 1}\left\|u^{j}(t)\right\|_{H^{4}}\right)>100 r_{0}$ sufficiently large, such that if $\left|x_{W}\right| \geq R_{\epsilon}$, then the following hold:

(1) There exists a unique smooth solution $W$ to (17.91) on the time interval $[0,1]$. Furthermore for any $0 \leq t \leq 1$ it has the decomposition

$$
W(t)=W^{1}(t)+W^{2}(t),
$$


where

$$
\begin{aligned}
& \operatorname{supp}\left(W^{1}(t)\right) \subset \Omega_{1}^{\epsilon}, \\
& \Omega_{1}^{\epsilon}:=\left\{x \in \mathbb{R}^{3}: d\left(x, \operatorname{supp}\left(\omega_{1}^{0}\right)\right)<r_{0}+\epsilon\right\}, \\
& \operatorname{supp}\left(W^{2}(t)\right) \subset \Omega_{W}^{\epsilon}, \\
& \Omega_{W}^{\epsilon}:=\left\{y=x+x_{W}: d\left(x, \operatorname{supp}\left(\omega_{2}^{0}\right)\right)<r_{0}+\epsilon\right\} .
\end{aligned}
$$

(2) The flow $W$ is uniformly close to $\omega^{1}(\cdot)+\omega^{2}\left(\cdot-x_{W}\right)$ :

$$
\begin{aligned}
& \max _{0 \leq t \leq 1}\left\|W^{1}(t, \cdot)-\omega^{1}(t, \cdot)\right\|_{H^{2}}<\epsilon, \\
& \max _{0 \leq t \leq 1}\left\|W^{2}(t, \cdot)-\omega^{2}\left(t, \cdot-x_{W}\right)\right\|_{H^{2}}<\epsilon
\end{aligned}
$$

(3) All higher Sobolev norms of $W^{1}$ and $W^{2}$ can be controlled in terms of $\omega_{0}^{1}$ and $\omega_{0}^{2}$ respectively: Let

$$
N=\max _{0 \leq t \leq 1}\left(\left\|\omega^{1}(t, \cdot)\right\|_{\infty}+\left\|\omega^{2}(t, \cdot)\right\|_{\infty}\right)+\left\|u_{0}^{1}\right\|_{2}+\left\|u_{0}^{2}\right\|_{2} .
$$

Here $u_{0}^{1}, u_{0}^{2}$ the velocity fields corresponding to the vorticity $\omega_{0}^{1}, \omega_{0}^{2}$ respectively. Then for any $k \geq 3$,

$$
\begin{aligned}
& \max _{0 \leq t \leq 1}\left\|W^{1}(t, \cdot)\right\|_{H^{k}} \leq C\left(k,\left\|\omega_{0}^{1}\right\|_{H^{k}}, N\right)<\infty \\
& \max _{0 \leq t \leq 1}\left\|W^{2}(t, \cdot)\right\|_{H^{k}} \leq C\left(k,\left\|\omega_{0}^{2}\right\|_{H^{k}}, N\right)<\infty .
\end{aligned}
$$

Proof of Lemma 7.13. Let $R=\left|x_{W}\right|$ and denote

$$
\begin{aligned}
& M_{0}=100\left(\left\|u_{0}^{1}\right\|_{H^{4}}+\left\|u_{0}^{2}\right\|_{H^{4}}\right), \\
& M_{1}=\max _{0 \leq t \leq 1}\left(\left\|\omega^{1}(t)\right\|_{\infty}+\left\|\omega^{2}(t)\right\|_{\infty}+1\right) .
\end{aligned}
$$

Consider the 3D Euler equation (in velocity formulation)

$$
\left\{\begin{array}{l}
\partial_{t} u+(u \cdot \nabla) u=-\nabla p \\
\nabla \cdot u=0 \\
\left.u\right|_{t=0}=u_{0} .
\end{array}\right.
$$

Suppose

$$
\left\|u_{0}\right\|_{H^{4}} \leq M_{0}
$$

and on some time interval $[0, \tau], \tau \leq 1$,

$$
\max _{0 \leq t \leq \tau}\|\omega(t)\|_{\infty} \leq M_{1}
$$

where $\omega=\operatorname{curl}(u)$. Then by a simple energy estimate, we have

$$
\max _{0 \leq t \leq \tau}\|u(t)\|_{H^{4}} \leq M_{2}
$$

where $M_{2}=M_{2}\left(M_{0}, M_{1}\right)>0$ can be taken as a constant which is uniform for all $\tau \leq 1$. We shall need this constant below. Also by standard local wellposedness theory, if for some $t_{0}$ we have $\left\|u\left(t_{0}\right)\right\|_{H^{4}} \leq M_{2}$, then there exists $\tau_{0}=\tau_{0}\left(M_{2}\right)>0$ such that the corresponding local solution has lifespan at least $\left[t_{0}, t_{0}+\tau_{0}\right]$ and

$$
\max _{t_{0} \leq t \leq t_{0}+\tau_{0}}\|u(t)\|_{H^{4}} \leq 2 M_{2}
$$

This fact will also be used below. 
Now let $0=t_{0}<t_{1}<\cdots<t_{L-1}<t_{L}=1$ be a partition of the time interval $[0,1]$ such that

$$
\max _{0 \leq i \leq L-1}\left(t_{i+1}-t_{i}\right)<\tau_{0} .
$$

We now inductively check the following

Claim: For each $i=0,1, \cdots, L$, there exists $R_{i}>0$ sufficiently large such that if $R>R_{i}$, then the following hold:

(1) $W(t)$ has the decomposition (7.92) for all $0 \leq t \leq t_{i}$.

(2)

$$
\begin{aligned}
& \max _{0 \leq t \leq t_{i}}\left\|W^{1}(t, \cdot)-\omega^{1}(t, \cdot)\right\|_{2}<R^{-\frac{1}{4}} \\
& \max _{0 \leq t \leq t_{i}}\left\|W^{2}(t, \cdot)-\omega^{2}\left(t, \cdot-x_{W}\right)\right\|_{2}<R^{-\frac{1}{4}}
\end{aligned}
$$

$$
\max _{0 \leq t \leq t_{i}}\|U(t)\|_{H^{4}} \leq M_{2} .
$$

Indeed the claim holds trivially for $i=0$. Now assume the claim holds for all $i \leq l-1(l \geq 1)$, and we need to prove the claim for $i=l$. Since $\left\|U\left(t_{l-1}\right)\right\|_{H^{4}} \leq M_{2}$, by our choice of $\tau_{0}, U(t)$ can be extended to $\left[t_{l-1}, t_{l}\right]$, and

$$
\begin{aligned}
\max _{0 \leq t \leq t_{l}}\|U(t)\|_{H^{4}} & \leq \max \left\{\max _{0 \leq t \leq t_{l-1}}\|U(t)\|_{H^{4}}, \max _{t_{l-1} \leq t \leq t_{l}}\|U(t)\|_{H^{4}}\right\} \\
& \leq 2 M_{2} .
\end{aligned}
$$

By the inductive assumption, we have

$$
W\left(t_{l-1}\right)=W^{1}\left(t_{l-1}\right)+W^{2}\left(t_{l-1}\right)
$$

and for $R$ sufficiently large,

$$
\operatorname{dist}\left(\operatorname{supp}\left(W^{1}\left(t_{l-1}\right)\right), \operatorname{supp}\left(W^{2}\left(t_{l-1}\right)\right)\right)>\frac{2}{3} R .
$$

By (7.100) and finite speed propagation, we then have for $R$ sufficiently large,

$$
\operatorname{dist}\left(\operatorname{supp}\left(W^{1}(t)\right), \operatorname{supp}\left(W^{2}(t)\right)\right)>\frac{1}{3} R, \quad \forall 0 \leq t \leq t_{l} .
$$

Denote by $U^{1}, U^{2}$ the velocity fields corresponding to the vorticity $W^{1}$ and $W^{2}$ respectively. By (7.101) and an argument similar to (5.10), it is not difficult to check that

$$
\max _{0 \leq t \leq t_{l}} \max _{|\alpha| \leq 3}\left\|D^{\alpha} U^{2}(t, \cdot)\right\|_{L^{\infty}\left(x \in \operatorname{supp}\left(W^{1}(t)\right)\right)} \leq R^{-\frac{1}{3}},
$$

where again we need to take $R$ sufficiently large (to kill some prefactors).

Now observe that

$$
\left\{\begin{array}{l}
\partial_{t} W^{1}+\left(U^{1} \cdot \nabla\right) W^{1}=\left(W^{1} \cdot \nabla\right) U^{1}-\left(U^{2} \cdot \nabla\right) W^{1}+\left(W^{1} \cdot \nabla\right) U^{2} \\
\partial_{t} \omega^{1}+\left(u^{1} \cdot \nabla\right) \omega^{1}=\left(\omega^{1} \cdot \nabla\right) u^{1}, \\
\left.W^{1}\right|_{t=0}=\left.\omega^{1}\right|_{t=0}=\omega_{0}^{1} .
\end{array}\right.
$$

Set $\eta=W^{1}-\omega^{1}, v=U^{1}-u^{1}$. Then clearly

$$
\begin{aligned}
\partial_{t} \eta+ & (v \cdot \nabla) W^{1}+\left(u^{1} \cdot \nabla\right) \eta \\
& =(\eta \cdot \nabla) U^{1}+\left(\omega^{1} \cdot \nabla\right) v-\left(U^{2} \cdot \nabla\right) W^{1}+\left(W^{1} \cdot \nabla\right) U^{2} .
\end{aligned}
$$


A simple $L^{2}$ estimate using (7.100) and (7.102) then gives for $0<t \leq t_{l}$ :

$$
\begin{aligned}
& \partial_{t}\left(\|\eta\|_{2}^{2}\right) \lesssim\|\eta\|_{2} \cdot\|v\|_{6} \cdot\left\|\nabla W^{1}\right\|_{3}+\|\eta\|_{2}^{2} \cdot\left\|\nabla U^{1}\right\|_{\infty} \\
& +\|\nabla v\|_{2} \cdot\|\eta\|_{2} \cdot\left\|\omega^{1}\right\|_{\infty}+R^{-\frac{1}{3}}\left\|\nabla W^{1}\right\|_{2} \cdot\|\eta\|_{2} \\
& +R^{-\frac{1}{3}}\left\|W^{1}\right\|_{2} \cdot\|\eta\|_{2} \\
& \lesssim_{M_{2}, \omega^{1}}\|\eta\|_{2}^{2}+R^{-\frac{1}{3}}\|\eta\|_{2} .
\end{aligned}
$$

Integrating in time up to $t_{l}$ and taking $R$ sufficiently large then gives

$$
\max _{0 \leq t \leq t_{l}}\|\eta(t)\|_{2}<R^{-\frac{1}{4}} .
$$

This settles (7.97) for $i=l$. The inequality (7.98) is proved similarly. Interpolating (7.97), (7.98) with (7.100) then easily yields that (see (7.95))

$$
\max _{0 \leq t \leq t_{l}}\|W(t)\|_{\infty} \leq M_{1} \text {. }
$$

Therefore by (7.96), we can upgrade the rough estimate (7.100) to (7.99) for $i=l$. By (7.97)-(7.98), interpolation and taking $R$ sufficiently large, we can easily have (see (7.90)

$$
\max _{0 \leq t \leq t_{l}}\|U(t, \cdot)\|_{\infty} \leq r_{0}+\epsilon / 2 .
$$

Hence the decomposition (7.92) follows. We have completely proved the claim.

By using the claim and a simple interpolation argument, it is not difficult to check that (7.93) holds. Finally (7.94) follows from a simple energy estimate using the disjointness of the support of $W^{1}$ and $W^{2}$ and an estimate similar to (7.102). The lemma is proved.

Proposition 7.14. Assume $\left\{\omega^{j}\right\}_{j=1}^{\infty}$ is a sequence of smooth functions each of which solves the $3 D$ incompressible Euler equation (in vorticity form)

$$
\left\{\begin{array}{l}
\partial_{t} \omega^{j}+\left(u^{j} \cdot \nabla\right) \omega^{j}=\left(\omega^{j} \cdot \nabla\right) u^{j}, \quad 0<t \leq 1, \\
u^{j}=-\Delta^{-1} \nabla \times \omega^{j} \\
\left.\omega^{j}\right|_{t=0}=\omega_{0}^{j} \in C_{c}^{\infty}\left(\mathbb{R}^{3}\right),
\end{array}\right.
$$

and satisfies the following condition:

For each $j \geq 1, \operatorname{supp}\left(\omega^{j}(t)\right) \subset B\left(0,2^{-10 j}\right)$ for any $0 \leq t \leq 1$ and

$$
\left\|u_{0}^{j}\right\|_{H^{\frac{5}{2}}}+\max _{0 \leq t \leq 1}\left(\left\|\omega^{j}(t)\right\|_{\infty}+\left\|u^{j}(t)\right\|_{\infty}\right) \leq 2^{-10 j} .
$$

Here $u_{0}^{j}$ is the velocity corresponding to the vorticity $\omega_{0}^{j}$.

Then there exist centers $x_{j} \in \mathbb{R}^{3}$ whose mutual distance are sufficiently large (i.e. $\left|x_{j}-x_{k}\right| \gg 1$ if $j \neq k$ ) such that the following hold:

(1) Take the initial data (vorticity)

$$
W_{0}(x)=\sum_{j=1}^{\infty} \omega_{0}^{j}\left(x-x_{j}\right)
$$

then $W_{0} \in L^{1} \cap L^{\infty} \cap H^{\frac{3}{2}} \cap C^{\infty}$. The corresponding initial velocity $U_{0} \in$ $H^{\frac{5}{2}} \cap C^{\infty}$. Furthermore for any $j \neq k$

$$
B\left(x_{j}, 100\right) \cap B\left(x_{k}, 100\right)=\varnothing .
$$


(2) With $W_{0}$ as initial data, there exists a unique smooth solution $W$ to the Euler equation (in vorticity form)

$$
\left\{\begin{array}{l}
\partial_{t} W+(U \cdot \nabla) W=(W \cdot \nabla) U \\
U=-\Delta^{-1} \nabla \times W \\
\left.W\right|_{t=0}=W_{0} .
\end{array}\right.
$$

on the time interval $[0,1]$ satisfying $W \in L_{t}^{\infty} L_{x}^{1} \cap L_{t}^{\infty} L_{x}^{\infty} \cap C^{\infty}, U \in$ $C^{\infty} \cap L_{t}^{\infty} L_{x}^{2}$. Moreover for any $0 \leq t \leq 1$,

$$
\operatorname{supp}(W(t, \cdot)) \subset \bigcup_{j=1}^{\infty} B\left(x_{j}, 1\right) .
$$

(3) For any $\epsilon>0$, there exists an integer $J_{\epsilon}$ sufficiently large such that if $j \geq J_{\epsilon}$, then

$$
\max _{0 \leq t \leq 1}\left\|W(t, \cdot)-\omega^{j}\left(t, \cdot-x_{j}\right)\right\|_{H^{2}\left(B\left(x_{j}, 1\right)\right)}<\epsilon .
$$

Proof of Proposition 7.14. Define $x_{1}=0$. By recursively applying Lemma 7.13, we can choose centers $x_{j}$ whose mutual distance is sufficiently large such that for each $l \geq 2$, we can find a unique smooth solution $W^{l}$ solving the system

$$
\left\{\begin{array}{l}
\partial_{t} W^{l}+\left(U^{l} \cdot \nabla\right) W^{l}=\left(W^{l} \cdot \nabla\right) U^{l}, \quad 0<t \leq 1, \\
U^{l}=-\Delta^{-1} \nabla \times W^{l}, \\
\left.W^{l}\right|_{t=0}=W_{0}^{l},
\end{array}\right.
$$

where

$$
W_{0}^{l}=\sum_{j=1}^{l} \omega_{0}^{j}\left(x-x_{j}\right)
$$

Furthermore $W^{l}$ satisfies

- $\operatorname{supp}\left(W^{l}(t)\right) \subset \bigcup_{j=1}^{l} B\left(x_{j}, \frac{1}{2}\right)$, for all $0 \leq t \leq 1$.

- $\max _{0 \leq t \leq 1}\left\|W^{l}(t, \cdot)-\omega^{j}\left(t, \cdot-x_{j}\right)\right\|_{H^{2}\left(B\left(x_{j}, 1\right)\right)}<2^{-j}$, for any $1 \leq j \leq l$.

- $\max _{0 \leq t \leq 1}\left\|W^{l+1}(t, \cdot)-W^{l}(t, \cdot)\right\|_{H^{2}\left(\bigcup_{j=1}^{l} B\left(x_{j}, 1\right)\right)}<2^{-l}$.

- $\max _{0 \leq t \leq 1}\left\|W^{l+1}(t, \cdot)-W^{l}(t, \cdot)\right\|_{L^{2}}<2^{-l}$.

- $\max _{0 \leq t \leq 1}\left\|W^{l}(t, \cdot)\right\|_{H^{k}\left(B\left(x_{j}, 1\right)\right)} \leq C_{k}=C_{k}\left(k,\left\|\omega_{0}^{j}\right\|_{H^{k}}\right)<\infty$, for any $1 \leq$ $j \leq l$.

Note that in the last inequality above we have no dependence on other constants thanks to the strong assumption (7.103).

Now define

$$
W(t, x)=\left\{\begin{array}{l}
\lim _{l \rightarrow \infty} W^{l}(t, x), \quad \text { if } x \in \bigcup_{j=1}^{\infty} B\left(x_{j}, 1\right), \\
0, \quad \text { otherwise. }
\end{array}\right.
$$

Fix any $j_{0} \geq 1$. By using the properties of $W^{l}$ listed above, we have

$$
\max _{0 \leq t \leq 1}\left\|W^{l+1}(t, \cdot)-W^{l}(t, \cdot)\right\|_{H^{2}\left(B\left(x_{j_{0}}, 1\right)\right)} \leq 2^{-l}, \quad \text { if } l \geq j_{0}+1 .
$$

Also for any $k \geq 3$,

$$
\max _{0 \leq t \leq 1}\left\|W^{l}(t, \cdot)\right\|_{H^{k}\left(B\left(x_{j_{0}}, 1\right)\right)} \leq C_{k}, \quad \forall l \geq j_{0}+1 .
$$


Therefore $\left(W^{l}\right)$ is Cauchy in $H^{k}\left(B\left(x_{j_{0}}, 1\right)\right)$ for any $k \geq 2$. Hence $W^{l}$ converges uniformly to $W \in C^{\infty}\left(\left(B\left(x_{j_{0}}, 1\right)\right)\right.$. Since $j_{0}$ is arbitrary, we obtain $W \in C^{\infty}\left(\mathbb{R}^{3}\right)$. Similarly fix any $j_{0} \geq 1$. By Sobolev embedding, we have

$$
\begin{aligned}
& \max _{0 \leq t \leq 1}\left\|W^{l}(t, \cdot)-\omega^{j_{0}}(t, \cdot)\right\|_{L^{\infty}\left(B\left(x_{j_{0}}, 1\right)\right)} \\
\lesssim & \max _{0 \leq t \leq 1}\left\|W^{l}(t, \cdot)-\omega^{j_{0}}(t, \cdot)\right\|_{H^{2}\left(B\left(x_{j_{0}}, 1\right)\right)} \lesssim 2^{-j_{0}}, \quad \forall l \geq j_{0}+1 .
\end{aligned}
$$

By (7.103) and sending $l \rightarrow \infty$, we obtain $\max _{0 \leq t \leq 1}\|W(t, \cdot)\|_{L^{\infty}} \lesssim 1$. Similarly it is also easy to check that $W \in L_{t}^{\infty} L_{x}^{1}$. Since $W^{l}$ is Cauchy in $L^{2}$, by Sobolev embedding we have $U^{l}$ is Cauchy in $L^{6}$ and converges to the limit $U$. It is not difficult to check that $U$ is smooth and $W$ is the desired solution. The estimate (7.105) follows obviously from the property of $W^{l}$ and passing $l$ to the limit. The proposition is proved.

We are now ready to complete the

Proof of Theorem 1.8. It suffices for us to prove the case $\omega_{0}^{(g)} \equiv 0$. The case for nonzero $\omega_{0}^{(g)}$ is a simple modification of the proof below.

For each $j \geq 1$, by using Proposition 7.12 we can find a smooth solution $\omega^{j}$ solving the system

$$
\left\{\begin{array}{l}
\partial_{t} \omega^{j}+\left(u^{j} \cdot \nabla\right) \omega^{j}=\left(\omega^{j} \cdot \nabla\right) u^{j}, \quad 0<t \leq 1, \\
u^{j}=-\Delta^{-1} \nabla \times \omega^{j}, \\
\left.\omega^{j}\right|_{t=0}=\omega_{0}^{j},
\end{array}\right.
$$

such that the following hold:

- $\operatorname{supp}\left(\omega^{j}(t, \cdot)\right) \subset\left\{x,|x|<2^{-100 j}\right\}$, for any $0 \leq t \leq 1$.

- $\max _{0 \leq t \leq 1}\left(\left\|\omega^{j}(t)\right\|_{L^{\infty}}+\left\|u^{j}(t)\right\|_{L^{\infty}}\right) \leq 2^{-100 j}$.

- Let $u_{0}^{j}$ be the velocity corresponding to the vorticity $\omega_{0}^{j}$, then

$$
\left\|u_{0}^{j}\right\|_{H^{\frac{5}{2}}}<2^{-100 j} \text {. }
$$

- For some $0<t_{j}^{0}<\frac{1}{j}$, we have

$$
\left\|\omega^{j}\left(t_{j}^{0}, \cdot\right)\right\|_{\dot{H}^{\frac{3}{2}}}>2^{j} .
$$

By continuity and the last inequality above, we can find $0<t_{j}^{1}<t_{j}^{2}<\frac{1}{j}$ such that

$$
\left\|\omega^{j}(t, \cdot)\right\|_{\dot{H}^{\frac{3}{2}}}>2^{j}, \quad \forall t_{j}^{1} \leq t \leq t_{j}^{2} .
$$

By Proposition 7.14 we can then find centers $x_{j}$ and build a smooth solution $W$ having initial data

$$
W(0, x)=\sum_{j=1}^{\infty} \omega_{0}^{j}\left(x-x_{j}\right) .
$$

The regularity properties of $W$ are simple consequences of Proposition 7.14 .

By (7.104), we can write

$$
W(t, x)=\sum_{j=1}^{\infty} W^{j}(t, x),
$$

where $W^{j} \in C_{c}^{\infty}\left(B\left(x_{j}, 1\right)\right)$. 
Now we make the following

Claim: there exists an integer $J_{1}>0$ and constants $C_{1}>0, C_{2}>0$ such that the following hold: for any $0 \leq \tau_{0} \leq 1$, if $\left\|W\left(\tau_{0}, \cdot\right)\right\|_{\dot{H}^{\frac{3}{2}}\left(\mathbb{R}^{3}\right)}<\infty$, then

$$
\left\|W\left(\tau_{0}, \cdot\right)\right\|_{\dot{H}^{\frac{3}{2}}} \geq C_{1}\left\|\omega^{j}\left(\tau_{0}, \cdot\right)\right\|_{\dot{H}^{\frac{3}{2}}}-C_{2}, \quad \forall j \geq J_{1} .
$$

Here the constant $C_{1}>0$ is actually an absolute constant. The constant $C_{2}$ depends on $\max _{0 \leq t \leq 1}\|W(t, \cdot)\|_{2}$.

To prove the claim, fix a smooth cut-off function $\phi \in C_{c}^{\infty}\left(\mathbb{R}^{3}\right)$ such that $\phi(x)=1$ for $|x| \leq 1$ and $\phi(x)=0$ for $|x| \geq 2$. Since $\left|x_{j}-x_{k}\right| \gg 1$ for $j \neq k$, by (7.104), we have for any $j \geq 1$, we have

$$
W^{j}\left(\tau_{0}, x\right)=W\left(\tau_{0}, x\right) \phi\left(x-x_{j}\right)=W\left(\tau_{0}, x\right) \phi_{j}(x), \quad \text { here } \phi_{j}(x):=\phi\left(x-x_{j}\right) .
$$

Fourier transform and the triangle inequality then give

$$
\begin{aligned}
|\xi|^{\frac{3}{2}}\left|\widehat{W^{j}}\left(\tau_{0}, \xi\right)\right| \lesssim|\xi|^{\frac{3}{2}} \int_{\mathbb{R}^{3}}\left|\hat{W}\left(\tau_{0}, \xi-\eta\right)\right|\left|\hat{\phi}_{j}(\eta)\right| d \eta \\
\lesssim \int_{\mathbb{R}^{3}}|\xi-\eta|^{\frac{3}{2}}\left|\hat{W}\left(\tau_{0}, \xi-\eta\right)\right|\left|\hat{\phi}_{j}(\eta)\right| d \eta \\
\quad+\int_{\mathbb{R}^{3}}\left|\hat{W}\left(\tau_{0}, \xi-\eta\right)\right||\eta|^{\frac{3}{2}}\left|\hat{\phi}_{j}(\eta)\right| d \eta
\end{aligned}
$$

Young's inequality then gives for any $j \geq 1$,

$$
\left\|W^{j}\left(\tau_{0}, \cdot\right)\right\|_{\dot{H}^{\frac{3}{2}}} \lesssim\left\|W\left(\tau_{0}, \cdot\right)\right\|_{\dot{H}^{\frac{3}{2}}}+\left\|W\left(\tau_{0}, \cdot\right)\right\|_{L^{2}} .
$$

Easy to check that the implied constants in the above inequalities are only absolute constants (they depend only on the cut-off function $\phi$ ). By (7.105) and choosing $\epsilon=1$, we get for any $j \geq J_{1}$,

$$
\left\|\omega^{j}\left(\tau_{0}, \cdot\right)\right\|_{\dot{H}^{\frac{3}{2}}} \leq \tilde{C}_{1}\left\|W\left(\tau_{0}, \cdot\right)\right\|_{\dot{H}^{\frac{3}{2}}}+\tilde{C}_{2},
$$

where $\tilde{C}_{1}>0$ is an absolute constant and $\tilde{C}_{2}$ depends only on $\max _{0 \leq t \leq 1}\|W(t, \cdot)\|_{L^{2}}$. The claim is proved.

With (7.107) in hand, we now argue by contradiction to finish the proof of the theorem. Assume for some $t_{0}<1$, we have

$$
L_{0}:=\operatorname{ess} \sup _{0 \leq t \leq t_{0}}\|W(t, \cdot)\|_{\dot{H}^{\frac{3}{2}}}<\infty .
$$

By (7.106), we choose $j \gg 1$ sufficiently large such that

$$
\begin{aligned}
& C_{1} 2^{j}-C_{2}>2 L_{0}, \\
& t_{j}^{2}<t_{0} .
\end{aligned}
$$

By (7.107), for any $t_{j}^{1} \leq t \leq t_{j}^{2}$, we must have

$$
2 L_{0} \leq\|W(t, \cdot)\|_{\dot{H}^{\frac{3}{2}}}<\infty, \quad \text { or }\|W(t, \cdot)\|_{\dot{H}^{\frac{3}{2}}}=+\infty .
$$

This obviously contradicts (7.108). The theorem is proved. 


\section{3D COMPACTLY SUPPORTED CASE}

Lemma 8.1. Let $f \in C_{c}^{\infty}(B(0,100)), g \in C_{c}^{\infty}(B(0,100))$ be axisymmetric functions on $\mathbb{R}^{3}$ having the form:

$$
f(x)=f^{\theta}(r, z) e_{\theta}, \quad g(x)=g^{\theta}(r, z) e_{\theta}, \quad x=\left(x_{1}, x_{2}, z\right), r=\sqrt{x_{1}^{2}+x_{2}^{2}},
$$

where $f^{\theta}$ and $g^{\theta}$ are scalar-valued and vanish near $r=0$, i.e. for some $r_{0}>0$,

$$
\begin{aligned}
& \operatorname{supp}\left(f^{\theta}\right) \subset\left\{(r, z): r>r_{0}\right\}, \\
& \operatorname{supp}\left(g^{\theta}\right) \subset\left\{(r, z): r>r_{0}\right\} .
\end{aligned}
$$

Let $\omega^{a}$ and $\omega$ be smooth solutions to the following axisymmetric (without swirl) Euler equations:

$$
\begin{aligned}
& \left\{\begin{array}{l}
\partial_{t}\left(\frac{\omega^{a}}{r}\right)+\left(u^{a} \cdot \nabla\right)\left(\frac{\omega^{a}}{r}\right)=0, \\
u^{a}=-\Delta^{-1} \nabla \times \omega^{a}, \\
\left.\omega^{a}\right|_{t=0}=f .
\end{array}\right. \\
& \left\{\begin{array}{l}
\partial_{t}\left(\frac{\omega}{r}\right)+(u \cdot \nabla)\left(\frac{\omega}{r}\right)=0, \\
u=-\Delta^{-1} \nabla \times \omega, \\
\left.\omega\right|_{t=0}=f+g .
\end{array}\right.
\end{aligned}
$$

For any $\epsilon>0$, there exists $\delta=\delta(\epsilon, f)>0$ sufficiently small such that if

$$
\|g\|_{\infty} \exp \left(C \cdot\left\|\frac{g}{r}\right\|_{L^{3,1}}\right)<\delta
$$

then

$$
\max _{0 \leq t \leq 1}\left\|\omega^{a}(t, \cdot)-\omega(t, \cdot)\right\|_{\infty}<\epsilon .
$$

Here in (8.3), $C>0$ is the same absolute constant as in the inequality

$$
\left\|\frac{u^{r}}{r}\right\|_{\infty} \leq C\left\|\frac{\omega}{r}\right\|_{L^{3,1}}
$$

Proof of Lemma 8.1. In this proof our main "smallness" parameter is $\|g\|_{\infty}$. To simplify the notations, we shall denote $X=O(\delta)$ if the quantity $X$ can be made arbitrarily small depending on $\|g\|_{\infty}$. For example we shall write $X=O(\delta)$ if $X$ satisfies the inequality of the following sort:

$$
|X| \lesssim f\|g\|_{\infty} \exp \left(C\left\|\frac{g}{r}\right\|_{L^{3,1}}\right) .
$$

Here $C>0$ is some absolute constant. Other inequalities similar to the above will all be denoted by the same notation $O(\delta)$ whenever there is no confusion. We shall denote $X=O(1)$ if

$$
X \lesssim_{f} 1 .
$$

We first decompose the solution to (8.2) as

$$
\omega=\omega^{1}+\omega^{2},
$$


where $\omega^{1}, \omega^{2}$ solve the linear systems

$$
\begin{aligned}
& \left\{\begin{array}{l}
\partial_{t}\left(\frac{\omega^{1}}{r}\right)+(u \cdot \nabla)\left(\frac{\omega^{1}}{r}\right)=0, \\
\left.\omega^{1}\right|_{t=0}=f,
\end{array}\right. \\
& \left\{\begin{array}{l}
\partial_{t}\left(\frac{\omega^{2}}{r}\right)+(u \cdot \nabla)\left(\frac{\omega^{2}}{r}\right)=0, \\
\left.\omega^{2}\right|_{t=0}=g .
\end{array}\right.
\end{aligned}
$$

Consider first (8.5). Since in (8.2), we have

$$
\begin{aligned}
\left\|\frac{\omega(t)}{r}\right\|_{L^{3,1}} & =\left\|\frac{\omega(0)}{r}\right\|_{L^{3,1}} \\
& \leq\left\|\frac{f}{r}\right\|_{L^{3,1}}+\left\|\frac{g}{r}\right\|_{L^{3,1}}, \quad \forall t \geq 0 .
\end{aligned}
$$

Recalling $u=u^{r} e_{r}+u^{z} e_{z}$, we get

$$
\begin{aligned}
\left\|\frac{u^{r}(t)}{r}\right\|_{\infty} & \lesssim\left\|\frac{\omega(t)}{r}\right\|_{L^{3,1}} \\
& \lesssim\left\|\frac{f}{r}\right\|_{L^{3,1}}+\left\|\frac{g}{r}\right\|_{L^{3,1}}, \quad \forall t \geq 0 .
\end{aligned}
$$

Rewrite (8.5) as

$$
\partial_{t} \omega^{2}+(u \cdot \nabla) \omega^{2}=\frac{u^{r}}{r} \omega^{2} .
$$

We obtain for some absolute constant $C>0$,

$$
\begin{aligned}
\max _{0 \leq t \leq 1}\left\|\omega^{2}(t)\right\|_{\infty} & \leq\|g\|_{\infty} \exp \left(C \max _{0 \leq t \leq 1}\left\|\frac{u^{r}(t)}{r}\right\|_{\infty}\right) \\
& \leq\|g\|_{\infty} \exp \left(C\left(\left\|\frac{f}{r}\right\|_{L^{3,1}}+\left\|\frac{g}{r}\right\|_{L^{3,1}}\right)\right) \\
& =O(\delta) .
\end{aligned}
$$

Thus we only need to control $\left\|\omega^{1}-\omega^{a}\right\|_{\infty}$.

Set $\eta=\omega^{a}-\omega^{1}$. Denote by $u^{1}, u^{2}$ the velocity fields corresponding to $\omega^{1}, \omega^{2}$ respectively. We first show that

$$
\max _{0 \leq t \leq 1}\|\eta(t, \cdot)\|_{2}=O(\delta) .
$$

Rewrite (8.4) as

$$
\partial_{t} \omega^{1}+\left(u^{1} \cdot \nabla\right) \omega^{1}=\left(\omega^{1} \cdot \nabla\right) u^{1}-\left(u^{2} \cdot \nabla\right) \omega^{1}+\left(\omega^{1} \cdot \nabla\right) u^{2} .
$$

By (8.1), we have

$$
\partial_{t} \omega^{a}+\left(u^{a} \cdot \nabla\right) \omega^{a}=\left(\omega^{a} \cdot \nabla\right) u^{a} .
$$

Therefore the equation for $\eta$ takes the form

$$
\begin{aligned}
& \partial_{t} \eta+\left(\left(u^{a}-u^{1}\right) \cdot \nabla\right) \omega^{a}+\left(u^{1} \cdot \nabla\right) \eta \\
& =(\eta \cdot \nabla) u^{a}+\left(\omega^{1} \cdot \nabla\right)\left(u^{a}-u^{1}\right) \\
& \quad+\left(u^{2} \cdot \nabla\right) \omega^{a}-\left(u^{2} \cdot \nabla\right) \eta-\left(\omega^{1} \cdot \nabla\right) u^{2} .
\end{aligned}
$$


Computing the $L^{2}$ norm then gives

$$
\begin{aligned}
\partial_{t}\left(\|\eta(t)\|_{2}^{2}\right) \lesssim & \left\|u^{a}-u^{1}\right\|_{6} \cdot\left\|D \omega^{a}\right\|_{3} \cdot\|\eta\|_{2}+\|\eta\|_{2}^{2} \cdot\left\|D u^{a}\right\|_{\infty} \\
& +\left\|\omega^{1}\right\|_{\infty} \cdot\left\|D\left(u^{a}-u^{1}\right)\right\|_{2} \cdot\|\eta\|_{2} \\
& +\left\|u^{2}\right\|_{6} \cdot\left\|D \omega^{a}\right\|_{3} \cdot\|\eta\|_{2}+\left\|\omega^{1}\right\|_{\infty} \cdot\left\|D u^{2}\right\|_{2} \cdot\|\eta\|_{2} \\
\lesssim & \left.\left\|D \omega^{a}\right\|_{3}+\left\|D u^{a}\right\|_{\infty}+\left\|\omega^{1}\right\|_{\infty}\right)\|\eta\|_{2}^{2} \\
& +\left(\left\|u^{2}\right\|_{6}\left\|D \omega^{a}\right\|_{3}+\left\|\omega^{1}\right\|_{\infty}\left\|D u^{2}\right\|_{2}\right)\|\eta\|_{2} \\
\lesssim( & \left.O(1)+\left\|\omega^{1}\right\|_{\infty}\right)\|\eta\|_{2}^{2} \\
& +\left(O(1) \cdot\left\|D u^{2}\right\|_{2}+\left\|\omega^{1}\right\|_{\infty}\left\|D u^{2}\right\|_{2}\right)\|\eta\|_{2} .
\end{aligned}
$$

By an estimate similar to (8.6), we have

$$
\max _{0 \leq t \leq 1}\left\|D u^{2}(t)\right\|_{2} \lesssim \max _{0 \leq t \leq 1}\left\|\omega^{2}(t)\right\|_{2}=O(\delta) .
$$

Similarly by Sobolev embedding,

$$
\max _{0 \leq t \leq 1}\left\|u^{2}(t)\right\|_{2} \lesssim \max _{0 \leq t \leq 1}\left\|\omega^{2}(t)\right\|_{\frac{6}{5}}=O(\delta) .
$$

This together with (8.6) gives

$$
\begin{aligned}
\max _{0 \leq t \leq 1}\left\|u^{2}(t)\right\|_{\infty} & \lesssim \max _{0 \leq t \leq 1}\left\|u^{2}(t)\right\|_{2}+\max _{0 \leq t \leq 1}\left\|\omega^{2}(t)\right\|_{\infty} \\
& =O(\delta) .
\end{aligned}
$$

By (8.4), we have

$$
\begin{aligned}
& \left\|\frac{\omega^{1}(t)}{r}\right\|_{L^{3,1}}=\left\|\frac{f}{r}\right\|_{L^{3,1}}=O(1), \quad \forall t \geq 0 \\
& \max _{0 \leq t \leq 1}\left\|\frac{\left(u^{1}(t)\right)^{r}}{r}\right\|_{\infty} \lesssim\left\|\frac{\omega^{1}(t)}{r}\right\|_{L^{3,1}}=O(1), \\
& \max _{0 \leq t \leq 1}\left\|\frac{\omega^{1}(t)}{r}\right\|_{\infty} \leq\left\|\frac{f}{r}\right\|_{\infty}=O(1) .
\end{aligned}
$$

Here we write $u^{1}=\left(u^{1}\right)^{r} e_{r}+\left(u^{1}\right)^{z} e_{z}$.

Rewrite (8.7) as

$$
\partial_{t} \omega^{1}+(u \cdot \nabla) \omega^{1}=\frac{\left(u^{1}\right)^{r}}{r} \omega^{1}+\left(u^{2}\right)^{r} \frac{\omega^{1}}{r} .
$$

Using (8.12) and (8.13), we get

$$
\max _{0 \leq t \leq 1}\left\|\omega^{1}(t)\right\|_{\infty}=O(1)
$$

Plugging (8.10) and (8.14) into (8.9), we obtain

$$
\max _{0 \leq t \leq 1}\|\eta(t, \cdot)\|_{2}=O(\delta) .
$$

By (8.14) - 8.15) and Hölder, we get

$$
\begin{aligned}
\max _{0 \leq t \leq 1}\left\|\omega^{a}(t)-\omega^{1}(t)\right\|_{4} & \lesssim \max _{0 \leq t \leq 1}\left(\|\eta(t)\|_{2}^{\frac{1}{2}}\|\eta(t)\|_{\infty}^{\frac{1}{2}}\right) \\
& =O(\delta)
\end{aligned}
$$


By $L^{2}$-conservation of velocity for (8.2), we have

$$
\begin{aligned}
\|u(t, \cdot)\|_{2}=\|u(0)\|_{2} & \lesssim\|f\|_{\dot{H}^{-1}}+\|g\|_{\dot{H}^{-1}} \\
& \lesssim\|f\|_{1}+\|f\|_{\infty}+\|g\|_{1}+\|g\|_{\infty} \\
& \lesssim\|f\|_{\infty}+\|g\|_{\infty}=O(1), \quad \forall t \geq 0 .
\end{aligned}
$$

Therefore by (8.11),

$$
\begin{aligned}
\max _{0 \leq t \leq 1}\left\|u^{a}(t)-u^{1}(t)\right\|_{2} & \lesssim \max _{0 \leq t \leq 1}\left(\left\|u^{a}(t)\right\|_{2}+\left\|u^{2}(t)\right\|_{2}+\|u(t)\|_{2}\right) \\
& =O(1) .
\end{aligned}
$$

By (8.16)-8.17) and interpolation, we get

$$
\begin{aligned}
\max _{0 \leq t \leq 1}\left\|u^{a}(t)-u^{1}(t)\right\|_{\infty} & \lesssim \max _{0 \leq t \leq 1}\left\|u^{a}(t)-u^{1}(t)\right\|_{2}^{\frac{1}{7}} \cdot\left\|\omega^{a}(t)-\omega^{1}(t)\right\|_{4}^{\frac{6}{7}} \\
& =O(\delta) .
\end{aligned}
$$

Now using (8.12), (8.13) and (8.18), we can rewrite (8.8) as

$$
\begin{aligned}
& \partial_{t} \eta+(u \cdot \nabla) \eta \\
=- & \left(\left(u^{a}-u^{1}\right) \cdot \nabla\right) \omega^{a}+(\eta \cdot \nabla) u^{a} \\
& \quad+\left(u^{a}-u^{1}\right)^{r} \frac{\omega^{1}}{r}+\left(u^{2} \cdot \nabla\right) \omega^{a}-\left(u^{2}\right)^{r} \frac{\omega^{1}}{r} \\
= & O(\delta)+O(1) \eta+O(\delta) \cdot O(1) .
\end{aligned}
$$

Obviously then

$$
\max _{0 \leq t \leq 1}\|\eta(t)\|_{\infty}=O(\delta)
$$

The lemma is proved.

We now state a proposition which gives the solvability of the 3D axisymmetric without swirl Euler equation for a special class of initial data (vorticity). In particular we allow initial vorticity (denote it by $\omega_{0}$ ) to carry infinite $\left\|\frac{\omega_{0}}{r}\right\|_{L^{3,1}}$ norm which is not covered by standard theory. The trade off here is that we need a precise control of $L^{\infty}$-norm in the sense of Lemma 8.1 .

Proposition 8.2. Suppose $\left\{g_{i}\right\}_{i=1}^{\infty}$ is a sequence of axisymmetric functions on $\mathbb{R}^{3}$ satisfying the following conditions:

- For each $i \geq 1, g_{i}(x)=g_{i}^{\theta}(r, z) e_{\theta}$, where $g_{i}^{\theta}$ is scalar-valued and vanishes near $r=0$ :

$$
\operatorname{supp}\left(g_{i}^{\theta}\right) \subset\left\{(r, z): r>r_{i}\right\}, \quad \text { for some } r_{i}>0 .
$$

- $g_{i} \in C_{c}^{\infty}(B(0,100))$ and $\left\|g_{i}\right\|_{\infty}<2^{-i}$.

- For each $i \geq 2$, denote $f_{i}=\sum_{j=1}^{i-1} g_{j}$, then

$$
\left\|g_{i}\right\|_{\infty} \exp \left(C\left\|\frac{g_{i}}{r}\right\|_{L^{3,1}}\right)<\delta_{i},
$$

where $\delta_{i}=\delta\left(2^{-i}, f_{i}\right)$ as defined in (8.3).

Let

$$
g=\sum_{i=1}^{\infty} g_{i}
$$


and consider the system

$$
\left\{\begin{array}{l}
\partial_{t} \omega+(u \cdot \nabla) \omega=(\omega \cdot \nabla) u, \quad 0<t \leq 1 \\
u=-\Delta^{-1} \nabla \times \omega \\
\left.\omega\right|_{t=0}=g
\end{array}\right.
$$

Then there exists a unique solution $\omega$ to (8.19) with the following properties:

(1) $\omega$ is compactly supported:

$$
\operatorname{supp}(\omega(t, \cdot)) \subset B\left(0, R_{0}\right), \quad \forall 0<t \leq 1 .
$$

Here $R_{0}>0$ is an absolute constant.

(2) $\omega \in C_{t}^{0} C_{x}^{0}\left([0,1] \times \overline{B\left(0, R_{0}\right)}\right), u \in C_{t}^{0} L_{x}^{2} \cap L_{t}^{\infty} L_{x}^{\infty}\left([0,1] \times \mathbb{R}^{3}\right)$. In fact $u \in C_{t}^{0} C_{x}^{\alpha}\left([0,1] \times \mathbb{R}^{3}\right)$ for any $0<\alpha<1$.

Proof of Proposition 8.2. For each $l \geq 1$, let $\omega^{l}$ be the solution to the system

$$
\left\{\begin{array}{l}
\partial_{t}\left(\frac{\omega^{l}}{r}\right)+\left(u^{l} \cdot \nabla\right)\left(\frac{\omega^{l}}{r}\right)=0, \quad 0<t \leq 1, \\
u^{l}=-\Delta^{-1} \nabla \times \omega^{l}, \\
\left.\omega^{l}\right|_{t=0}=\sum_{i=1}^{l} g_{i} .
\end{array}\right.
$$

By Lemma 8.1 and the assumptions on $g_{i}$, we have

$$
\max _{0 \leq t \leq 1}\left\|\omega^{l+1}(t)-\omega^{l}(t)\right\|_{\infty}<2^{-l} .
$$

Noting that

$$
\begin{aligned}
\max _{0 \leq t \leq 1}\left\|\omega^{1}(t)\right\|_{\infty} & \lesssim\left\|g_{1}\right\|_{\infty} \exp \left(\text { Const } \cdot\left\|\frac{g_{1}}{r}\right\|_{L^{3,1}}\right) \\
& \lesssim 1
\end{aligned}
$$

we obtain

$$
\sup _{l \geq 1} \max _{0 \leq t \leq 1}\left\|\omega^{l}(t)\right\|_{\infty} \lesssim 1
$$

By energy conservation, we have

$$
\begin{aligned}
\left\|u^{l}(t)\right\|_{2} & =\left\|u^{l}(0)\right\|_{2} \lesssim\left\|\omega^{l}(0)\right\|_{1}+\left\|\omega^{l}(0)\right\|_{\infty} \\
& \lesssim 1, \quad \forall t \geq 0, l \geq 1 .
\end{aligned}
$$

Therefore

$$
\sup _{l \geq 1} \max _{0 \leq t \leq 1}\left\|u^{l}(t)\right\|_{\infty} \lesssim 1 .
$$

This shows that for some absolute constant $R_{0}>0$, we have

$$
\omega^{l}(t) \in C_{c}^{\infty}\left(B\left(0, R_{0}\right)\right), \quad \forall 0<t \leq 1, l \geq 1 .
$$

By (8.20), the sequence $\omega^{l}$ is Cauchy in the Banach space $C_{t}^{0} C_{x}^{0}\left([0,1] \times \overline{B\left(0, R_{0}\right)}\right)$ and hence converges to the limit point $\omega$ in the same space. By interpolation and Sobolev embedding it is not difficult to check that $u^{l}$ converges to $u \in C_{t}^{0} L_{x}^{2}$. By Sobolev embedding we get $u \in L_{t}^{\infty} L_{x}^{\infty} \cap C_{t}^{0} C_{x}^{\alpha}$ for any $\alpha<1$. It is not difficult to check that $\omega$ is the desired solution. The proposition is proved. 
We now take a parameter $A \gg 1$ and define

$$
\begin{aligned}
\tilde{g}_{A}\left(x_{1}, x_{2}, z\right) & =\tilde{g}_{A}(r, z) \\
& =\frac{\sqrt{\log A}}{\sqrt{A}} \sum_{A \leq k \leq A+\sqrt{A}} \eta_{k}(r, z),
\end{aligned}
$$

where $\eta_{k}$ is the same as in (7.12). Note the slight difference between $\tilde{g}_{A}$ and $g_{A}$ defined in (7.11). The main reason of choosing $\tilde{g}_{A}$ is that in the perturbation theory later we need better control of higher Sobolev norms of the solution, i.e. estimates like $\left\|\tilde{g}_{A}\right\|_{W^{1, q}} \lesssim 2^{A+}$, for all $3<q \leq \infty$. In comparison $\left\|g_{A}\right\|_{W^{1, q}} \sim 2^{2 A}$ since there we are summing $\eta_{k}$ over $k \leq 2 A$. This is why the modification is needed.

By a derivation similar to (7.14)-(7.18), easy to check

$$
\begin{aligned}
& \left\|\tilde{g}_{A} e_{\theta}\right\|_{\dot{B}_{2,1}^{\frac{3}{2}}\left(\mathbb{R}^{3}\right)}+\left\|\frac{\tilde{g}_{A}}{r} e_{\theta}\right\|_{L^{3,1}\left(\mathbb{R}^{3}\right)} \lesssim \sqrt{\log A}, \\
& \left\|\tilde{g}_{A}\right\|_{\dot{H}^{\frac{3}{2}}\left(\mathbb{R}^{3}\right)}+\left\|\tilde{g}_{A} e_{\theta}\right\|_{\dot{H}^{\frac{3}{2}\left(\mathbb{R}^{3}\right)}} \lesssim \frac{\sqrt{\log A}}{A^{\frac{1}{4}}}, \\
& \left\|\tilde{g}_{A}\right\|_{L^{p}\left(\mathbb{R}^{3}\right)}+\left\|\tilde{g}_{A} e_{\theta}\right\|_{L^{p}\left(\mathbb{R}^{3}\right)} \lesssim \frac{\sqrt{\log A}}{\sqrt{A}} \cdot 2^{-\frac{3 A}{p}}, \quad \forall 1 \leq p \leq \infty, \\
& \left\|D\left(\tilde{g}_{A} e_{\theta}\right)\right\|_{L^{p}\left(\mathbb{R}^{3}\right)} \lesssim \sqrt{\log A} \cdot 2^{\left(1-\frac{3}{p}\right)(A+\sqrt{A})}, \quad \forall 3 \leq p \leq \infty .
\end{aligned}
$$

These estimates will be needed later.

Lemma 8.3. Let $\omega$ be a smooth solution to the following system (written in axisymmetric vorticity form)

$$
\left\{\begin{array}{l}
\partial_{t}\left(\frac{\omega}{r}\right)+\left(u+u^{e x}\right) \cdot \nabla\left(\frac{\omega}{r}\right)=0, \quad 0<t \leq 1, r=\sqrt{x_{1}^{2}+x_{2}^{2}}, x=\left(x_{1}, x_{2}, z\right), \\
u=-\Delta^{-1} \nabla \times \omega, \\
\left.\omega\right|_{t=0}=\tilde{g}_{A} e_{\theta},
\end{array}\right.
$$

where $\tilde{g}_{A}$ was the same as in (8.21) and $u^{e x}$ is a given axisymmetric velocity field having the form (note that it is incompressible)

$$
u^{e x}(t, r, z)=a(t) r e_{r}-2 a(t) z e_{z} .
$$

Assume for some constant $B_{0}>0$,

$$
\sup _{0 \leq t \leq 1}|a(t)| \leq B_{0}<\infty .
$$

Let $\phi=\left(\phi^{r}, \phi^{z}\right)$ be the forward characteristic lines associated with the velocity $u+u^{\text {ex }}$ (see (7.2) ) and let $\tilde{\phi}$ be the corresponding inverse map. Then there exists $A_{0}=A_{0}\left(B_{0}\right)>0$ such that if $A>A_{0}$, then

$$
\max _{0 \leq t \leq \frac{1}{\log \log A}}\|(D \tilde{\phi})(t, \cdot)\|_{\infty}>\log \log A .
$$

Proof of Lemma 8.3. We shall give a slightly simpler proof than that given in Proposition 7.9. The idea is to take full advantage of the symmetry assumption and the fact that the off-diagonal terms of $D u$ vanishes completely at $(r, z)=(0,0)$. Assume (8.25) does not hold. By a derivation similar to (7.88)-(7.89), we have

$$
\max _{0 \leq t \leq \frac{1}{\log \log A}}\left(\|(D \tilde{\phi})(t, \cdot)\|_{\infty}+\|(D \phi)(t, \cdot)\|_{\infty}\right) \lesssim(\log \log A)^{2} .
$$


By (8.21) and (7.13), observe that $\tilde{g}_{A}$ is an odd function of $z$. Denote $v(t, r, z)=$ $u+u^{e x}$ and

$$
\begin{aligned}
& u^{\mathrm{ex}}=\left(u^{\mathrm{ex}}\right)^{r} e_{r}+\left(u^{\mathrm{ex}}\right)^{z} e_{z}, \\
& v=v^{r} e_{r}+v^{z} e_{z} .
\end{aligned}
$$

Easy to check that for any $t \geq 0, \omega(t)$ remains an odd function of $z$, and also

$$
\begin{aligned}
& v^{r}(t, 0, z)=v^{z}(t, r, 0)=0, \\
& \phi^{r}(t, 0, z)=\phi^{z}(t, r, 0)=0, \quad \forall r \geq 0, z \in \mathbb{R}, t \geq 0 .
\end{aligned}
$$

Clearly then

$$
\begin{aligned}
& \left(\partial_{r} v^{z}\right)(t, 0,0) \equiv 0 \equiv\left(\partial_{z} v^{r}\right)(t, 0,0), \\
& \left(\partial_{r} \phi^{z}\right)(t, 0,0) \equiv 0 \equiv\left(\partial_{z} \phi^{r}\right)(t, 0,0), \quad \forall t \geq 0 .
\end{aligned}
$$

Since $v$ is divergence-free (see $(\underline{8.23})$ ), we have

$$
2\left(\partial_{r} v^{r}\right)(t, 0,0)+\left(\partial_{z} v^{z}\right)(t, 0,0) \equiv 0, \quad \forall t \geq 0 .
$$

From the above identities, we then easily obtain

$$
\begin{aligned}
& \left(\partial_{r} \phi^{r}\right)(t, 0,0)=e^{\int_{0}^{t}\left(\partial_{r} v^{r}\right)(s, 0,0) d s} \\
& \left(\partial_{z} \phi^{z}\right)(t, 0,0)=e^{\int_{0}^{t}\left(\partial_{z} v^{z}\right)(s, 0,0) d s}=e^{-2 \int_{0}^{t}\left(\partial_{r} v^{r}\right)(s, 0,0) d s} .
\end{aligned}
$$

By (7.27) (easy to check that same estimate holds with $g_{A}$ replaced by $\tilde{g}_{A}$ ), (8.26) and (8.24), we get

$$
\begin{aligned}
\left(\partial_{r} v^{r}\right)(t, 0,0) & =\left(\partial_{r} u^{r}\right)(t, 0,0)+\left(\partial_{r}\left(u^{e x}\right)^{r}\right)(t, 0,0) \\
& \gtrsim \sqrt{\log A}(\log \log A)^{-16}-B_{0} .
\end{aligned}
$$

Plugging this into (8.27) then gives us

$$
\left(\partial_{r} \phi^{r}\right)(t, 0,0) \gtrsim e^{t \frac{\sqrt{\log A}}{(\log \log A)^{16}}-2 B_{0}} .
$$

This obviously contradicts (8.26) for $t=\frac{1}{\log \log A}$ and $A$ sufficiently large.

Lemma 8.4 (Control of the support). Let $\omega$ be a smooth solution to the following system (written in axisymmetric vorticity form)

$$
\left\{\begin{array}{l}
\partial_{t}\left(\frac{\omega}{r}\right)+\left(u+u_{1}+u_{2}\right) \cdot \nabla\left(\frac{\omega}{r}\right)=0, \quad 0<t \leq 1, r=\sqrt{x_{1}^{2}+x_{2}^{2}}, x=\left(x_{1}, x_{2}, z\right), \\
u=-\Delta^{-1} \nabla \times \omega \\
\left.\omega\right|_{t=0}=\tilde{g}_{A} e_{\theta}
\end{array}\right.
$$

where the following conditions hold:

- $\tilde{g}_{A}$ is the same as in (8.21);

- $u_{1}$ and $u_{2}$ are given smooth incompressible axisymmetric vector fields having the form

$$
\begin{aligned}
& u_{1}=a(t) r e_{r}-2 a(t) z e_{z} \\
& u_{2}=u_{2}^{r} e_{r}+u_{2}^{z} e_{z}
\end{aligned}
$$


and for some constant $B>0$,

$$
\begin{aligned}
& \sup _{0 \leq t \leq 1}|a(t)| \leq B, \\
& \sup _{0 \leq t \leq 1}|| \frac{u_{2}^{r}(t)}{r} \|_{\infty} \leq B, \\
& \left|u_{2}(t, x)\right| \leq B|x|^{2}, \quad \forall x \in \mathbb{R}^{2}, \quad 0 \leq t \leq 1 .
\end{aligned}
$$

Then there exists a constant $A_{0}=A_{0}(B)>0$ sufficiently large such that if $A>A_{0}$, then for any $0 \leq t \leq 1$,

$$
\operatorname{supp}(\omega(t, \cdot)) \subset B(0, R), \quad \text { with } R \leq C_{1} \cdot 2^{-A},
$$

where $C_{1}>0$ is a constant depending on $B$.

Also for any $0 \leq t \leq \frac{1}{\log \log A}$, we have

$$
\operatorname{supp}(\omega(t, \cdot)) \subset B(0, R), \quad \text { with } R \sim 2^{-A},
$$

where the implied constants (in $R \sim 2^{-A}$ ) are absolute constants.

Proof of Lemma 8.4. Denote $v=u+u_{1}+u_{2}$ and write

$$
\begin{aligned}
& v=v^{r} e_{r}+v^{z} e_{z}, \\
& u=u^{r} e_{r}+u^{z} e_{z} .
\end{aligned}
$$

By $L^{3,1}$ conservation of $\frac{\omega}{r}$ and (8.22), we have

$$
\sup _{0 \leq t \leq 1}\left\|\frac{u^{r}(t)}{r}\right\|_{\infty} \lesssim\left\|\frac{\omega(t=0, \cdot)}{r}\right\|_{L^{3,1}} \lesssim \sqrt{\log A} .
$$

By (8.28), we get

$$
\sup _{0 \leq t \leq 1}\left\|\frac{v^{r}(t)}{r}\right\|_{\infty} \lesssim B+\sqrt{\log A} .
$$

Rewrite the equation for $\omega$ as

$$
\partial_{t} \omega+(v \cdot \nabla) \omega=\frac{v^{r}}{r} \omega .
$$

By (8.31), a simple $L^{p}$-estimate (note that $v$ is incompressible) then gives

$$
\begin{aligned}
& \sup _{0 \leq t \leq 1}\|\omega(t)\|_{2} \lesssim e^{C(B+\sqrt{\log A})}\left\|g_{A}\right\|_{2}, \\
& \sup _{0 \leq t \leq 1}\|\omega(t)\|_{4} \lesssim e^{C(B+\sqrt{\log A})}\left\|g_{A}\right\|_{4},
\end{aligned}
$$

where $C>0$ is an absolute constant.

Note that by (8.22), we have

$$
\begin{aligned}
\left\|\tilde{g}_{A}\right\|_{2} & \lesssim \frac{\sqrt{\log A}}{\sqrt{A}} 2^{-\frac{3}{2} A}, \\
\left\|\tilde{g}_{A}\right\|_{4} & \lesssim \frac{\sqrt{\log A}}{\sqrt{A}} 2^{-\frac{3}{4} A} .
\end{aligned}
$$


By (8.32) and interpolation, we then get

$$
\begin{aligned}
\sup _{0 \leq t \leq 1}\|u(t)\|_{\infty} & \lesssim \sup _{0 \leq t \leq 1}\|\omega(t)\|_{2}^{\frac{1}{3}} \cdot\|\omega(t)\|_{4}^{\frac{2}{3}} \\
& \lesssim e^{C(B+\sqrt{\log A})} \cdot \frac{\sqrt{\log A}}{A} \cdot 2^{-A} \\
& <A^{-\frac{1}{3}} 2^{-A}
\end{aligned}
$$

where in the last inequality we need to take $A$ sufficiently large.

Denote $\phi$ as the (usual Euclidean) characteristic line associated with the velocity $v$. Then by (8.28) and (8.33), we get

$$
\frac{d}{d t}(|\phi(t)|) \lesssim A^{-\frac{1}{3}} 2^{-A}+B|\phi(t)|+B|\phi(t)|^{2} .
$$

Since $|\phi(0)| \lesssim 2^{-A}$, obviously (8.29) and (8.30) follows (in the latter case since $t \leq \frac{1}{\log \log A}$ we can take $A$ sufficiently large to kill pre-factors).

Lemma 8.5. Let $\omega$ be a smooth solution to the following system (written in axisymmetric vorticity form)

$$
\left\{\begin{array}{l}
\partial_{t}\left(\frac{W}{r}\right)+\left(U+u_{1}+u_{2}\right) \cdot \nabla\left(\frac{W}{r}\right)=0, \quad 0<t \leq 1, r=\sqrt{x_{1}^{2}+x_{2}^{2}}, x=\left(x_{1}, x_{2}, z\right), \\
U=-\Delta^{-1} \nabla \times W \\
\left.W\right|_{t=0}=\tilde{g}_{A} e_{\theta}
\end{array}\right.
$$

where the following conditions hold:

- $\tilde{g}_{A}$ is the same as in (8.21);

- $u_{1}$ and $u_{2}$ are given smooth incompressible axisymmetric vector fields having the form

$$
\begin{aligned}
& u_{1}=a(t) r e_{r}-2 a(t) z e_{z}, \\
& u_{2}=u_{2}^{r} e_{r}+u_{2}^{z} e_{z}
\end{aligned}
$$

and for some constant $B>0$,

$$
\begin{aligned}
& \sup _{0 \leq t \leq 1}|a(t)| \leq B \\
& \left|u_{2}(t, x)\right| \leq B \cdot|x|^{2} \\
& \left|\left(D u_{2}\right)(t, x)\right| \leq B \cdot|x| \\
& \left|\left(D^{2} u_{2}\right)(t, x)\right| \leq B, \quad \forall x \in \mathbb{R}^{3}, 0 \leq t \leq 1 .
\end{aligned}
$$

Let $\Phi=\left(\Phi^{r}, \Phi^{z}\right)$ be the characteristic line associated with the velocity field $U+$ $u_{1}+u_{2}$ (see (7.2) ) and let $\tilde{\Phi}$ be the corresponding inverse map.

There exists a constant $A_{0}=A_{0}(B)>0$ sufficiently large such that if $A>A_{0}$, then either

$$
\max _{0 \leq t \leq \frac{1}{\log \log A}}\|W(t, \cdot)\|_{\dot{H}^{\frac{3}{2}}}>\log \log \log A
$$

or

$$
\max _{0 \leq t \leq \frac{1}{\log \log A}}\|(D \tilde{\Phi})(t, \cdot)\|_{\infty}>\log \log \log A
$$


Proof of Lemma 8.5. By Lemma 8.4 and (8.34), we have $\operatorname{supp}(W(t, \cdot)) \subset\{x:|x| \lesssim$ $\left.2^{-A}\right\}$ and

$$
\begin{aligned}
& \left\|u_{2}(t, \cdot)\right\|_{L^{\infty}(\operatorname{supp}(W(t, \cdot)))} \lesssim 4^{-A}, \quad \forall 0 \leq t \leq 1, \\
& \left\|\left(D u_{2}\right)(t, \cdot)\right\|_{L^{\infty}(\operatorname{supp}(W(t, \cdot)))} \lesssim 2^{-A}, \quad \forall 0 \leq t \leq 1, \\
& \left\|\left(D^{2} u_{2}\right)(t, \cdot)\right\|_{L^{\infty}(\operatorname{supp}(W(t, \cdot)))} \lesssim 1, \quad \forall 0 \leq t \leq 1 .
\end{aligned}
$$

Throughout this proof we suppress the dependence of the implied constants on $B$ since $A$ will be taken sufficiently large.

Assume that both (8.35) and (8.36) do not hold, i.e.

$$
\max _{0 \leq t \leq \frac{1}{\log \log A}}\|W(t, \cdot)\|_{\dot{H}^{\frac{3}{2}}}+\max _{0 \leq t \leq \frac{1}{\log \log A}}\|(D \tilde{\Phi})(t, \cdot)\|_{\infty} \lesssim \log \log \log A .
$$

Easy to check that

$$
\max _{0 \leq t \leq \frac{1}{\log \log A}}\|(D \Phi)(t, \cdot)\|_{\infty} \lesssim(\log \log \log A)^{2} .
$$

We shall derive a contradiction. The idea is to compare $W$ with the other solution $\omega$ to the following "unperturbed" system

$$
\left\{\begin{array}{l}
\partial_{t}\left(\frac{\omega}{r}\right)+\left(u+u_{1}\right) \cdot \nabla\left(\frac{\omega}{r}\right)=0, \quad 0<t \leq 1, r=\sqrt{x_{1}^{2}+x_{2}^{2}}, x=\left(x_{1}, x_{2}, z\right), \\
u=-\Delta^{-1} \nabla \times \omega \\
\left.\omega\right|_{t=0}=\tilde{g}_{A} e_{\theta} .
\end{array}\right.
$$

By using the conservation of $\left\|\frac{W}{r}\right\|_{L^{3,1}}$ and $\left\|\frac{\omega}{r}\right\|_{L^{3,1}}$ respectively, it is not difficult to check that

$$
\begin{aligned}
& \sup _{0 \leq t \leq 1}\left\|\frac{U^{r}(t)}{r}\right\|_{\infty} \lesssim \sqrt{\log A}, \\
& \sup _{0 \leq t \leq 1}\left\|\frac{u^{r}(t)}{r}\right\|_{\infty} \lesssim \sqrt{\log A}, \\
& \sup _{0 \leq t \leq 1}\|W(t)\|_{q} \lesssim \frac{\sqrt{\log A}}{\sqrt{A}} 2^{-\frac{3 A}{q}}, \quad \forall 1<q \leq \infty, \\
& \sup _{0 \leq t \leq 1}\|\omega(t)\|_{q} \lesssim \frac{\sqrt{\log A}}{\sqrt{A}} 2^{-\frac{3 A}{q}}, \quad \forall 1<q \leq \infty,
\end{aligned}
$$

where in the last two inequalities we have used (8.37).

We carry out the perturbation argument in several steps.

Step 1. Set $\eta=\omega-W$. We first show that

$$
\|\eta(t, \cdot)\|_{B_{\infty, 1}^{0}} \lesssim 2^{-\frac{A}{2}+}, \quad \forall 0 \leq t \leq \frac{1}{\log \log A} .
$$

Here and below we use the notation $X+$ as in (2.1).

Rewrite the equations for $\omega$ and $W$ as

$$
\begin{aligned}
& \partial_{t} \omega+\left(u+u_{1}\right) \cdot \nabla \omega=(\omega \cdot \nabla)\left(u+u_{1}\right), \\
& \partial_{t} W+\left(U+u_{1}+u_{2}\right) \cdot \nabla W=(W \cdot \nabla)\left(U+u_{1}+u_{2}\right) .
\end{aligned}
$$

Taking the difference, we have

$$
\begin{aligned}
\partial_{t} \eta & +\left(u+u_{1}\right) \cdot \nabla \eta+\left(u-U-u_{2}\right) \cdot \nabla W \\
& =(\eta \cdot \nabla)\left(u+u_{1}\right)+(W \cdot \nabla)\left(u-U-u_{2}\right) .
\end{aligned}
$$


Let $1<p<3$. By (8.38) and (8.40), we have

$$
\begin{aligned}
& \partial_{t}\left(\|\eta\|_{p}\right) \lesssim \| u-U\left\|_{\left(\frac{1}{p}-\frac{1}{3}\right)^{-1}}\right\| D W\left\|_{3}+\right\| D W \|_{p} \cdot 4^{-A} \cdot B \\
&+\|\eta\|_{p} \cdot\left(\left\|\frac{u^{r}}{r}\right\|_{\infty}+B\right) \\
&+\|D(u-U)\|_{p} \cdot\|W\|_{\infty}+\|W\|_{p} \cdot 2^{-A} \\
& \lesssim\|\eta\|_{p} \cdot \log \log \log A+\log \log \log A \cdot 4^{-A} \cdot B \\
&+\|\eta\|_{p} \cdot(\sqrt{\log A}+B)+\|\eta\|_{p} \cdot \frac{\sqrt{\log A}}{\sqrt{A}}+4^{-A}
\end{aligned}
$$

Set $\eta(0)=0$, integrating in $t \leq \frac{1}{\log \log A}$ then gives

$$
\max _{0 \leq t \leq \frac{1}{\log \log A}}\|\eta(t, \cdot)\|_{p} \lesssim 4^{-A+}, \quad \forall 1<p<3 .
$$

This estimate is particularly good for $p=3-$.

Now for any $1<q<\infty$, a standard energy estimate using (8.22), (8.37) and (8.40) (using $\|W\|_{\infty}$ and $\|\omega\|_{\infty}$ ) gives for any $0 \leq t \leq 1$,

$$
\begin{aligned}
& \|W(t, \cdot)\|_{W^{1, q}} \lesssim 2^{A-}, \\
& \|\omega(t, \cdot)\|_{W^{1, q}} \lesssim 2^{A-}
\end{aligned}
$$

and obviously

$$
\max _{0 \leq t \leq 1}\|\eta(t, \cdot)\|_{W^{1, q}} \lesssim 2^{A-}, \quad \forall 1<q<\infty .
$$

Interpolating the above (set $q=\infty-$ ) with (8.42) (set $p=3-$ ) then gives (8.41).

Step 2. Let $\phi=\left(\phi^{r}, \phi^{z}\right)$ be the characteristic line associated with the velocity field $u+u_{1}$. We show that

$$
\max _{0 \leq t \leq \frac{1}{\log \log A}}\|\phi(t, \cdot)-\Phi(t, \cdot)\|_{\infty} \lesssim 2^{-\frac{7}{6} A+} .
$$

Set $Y(t)=\phi(t)-\Phi(t)$. By Lemma 8.4 we only need to consider the region $|x| \lesssim 2^{-A}$. By (8.34), we have the estimate

$$
\left|u_{2}(t, \Phi(t, r, z))\right| \lesssim 4^{-A}, \quad \forall 0 \leq t \leq \frac{1}{\log \log A}, \forall \sqrt{r^{2}+z^{2}} \lesssim 2^{-A}
$$

Let $Y(t)=\phi(t)-\Phi(t)=\left(Y^{r}(t), Y^{z}(t)\right)$. In order not to confuse the notation, we denote

$$
\begin{aligned}
& v=\left(u^{r}, u^{z}\right), \quad v_{1}=\left(u_{1}^{r}, u_{1}^{z}\right), \\
& V=\left(U^{r}, U^{z}\right), \quad v_{2}=\left(u_{2}^{r}, u_{2}^{z}\right) .
\end{aligned}
$$

Then the equation for $Y$ takes the form

$$
\frac{d}{d t} Y=\left(v+v_{1}\right)(\phi)-\left(v+v_{1}\right)(\Phi)+(v-V)(\Phi)-v_{2}(\Phi) .
$$

By (8.40) and a simple energy estimate, we have

$$
\begin{aligned}
\|D u\|_{\infty} & \lesssim\|\omega\|_{2}+\|\omega\|_{\infty} \log \left(10+\|\omega\|_{H^{2}}\right) \\
& \lesssim 1+\frac{\sqrt{\log A}}{\sqrt{A}} \cdot A \lesssim \sqrt{A} \cdot \sqrt{\log A} .
\end{aligned}
$$


Since $u(t, 0,0, z)=u^{z} e_{z}$, we have

$$
\begin{aligned}
\left|\frac{1}{r} u^{r}(t, r, z)\right| & =\left|\frac{\left(u\left(t, x_{1}, x_{2}, z\right)-u(t, 0,0, z)\right) \cdot e_{r}}{r}\right| \\
& \lesssim\|D u\|_{\infty} \lesssim \sqrt{A} \cdot \sqrt{\log A} .
\end{aligned}
$$

By the incompressibility condition $\partial_{r} u^{r}=-\frac{1}{r} u^{r}-\partial_{z} u^{z}$, we obtain

$$
\left\|\partial_{r} u^{r}\right\|_{\infty} \lesssim\|D u\|_{\infty} \lesssim \sqrt{A} \cdot \sqrt{\log A}
$$

Similarly we have the estimate for $\left\|\partial_{z} u^{r}\right\|_{\infty},\left\|\partial_{r} u^{z}\right\|_{\infty},\left\|\partial_{z} u^{z}\right\|_{\infty}$, and hence

$$
\|D v\|_{\infty} \lesssim \sqrt{A} \cdot \sqrt{\log A} .
$$

By (8.40), (8.41) and interpolation, we have

$$
\begin{aligned}
\max _{0 \leq t \leq \frac{1}{\log \log A}}\|u(t)-U(t)\|_{\infty} & \lesssim \max _{0 \leq t \leq \frac{1}{\log \log A}}\left(\|\omega(t)\|_{2}+\|W(t)\|_{2}\right)^{\frac{2}{3}}\|\omega(t)-W(t)\|_{\infty}^{\frac{1}{3}} \\
& \lesssim 2^{-A+} \cdot 2^{-\frac{A}{6}+} \\
& \lesssim 2^{-\frac{7}{6} A+} .
\end{aligned}
$$

Therefore

$$
\max _{0 \leq t \leq \frac{1}{\log \log A}}\|v(t)-V(t)\|_{\infty} \lesssim 2^{-\frac{7}{6} A+} .
$$

Plugging the estimates (8.47)-(8.48) into (8.45) and using (8.44), we have

$$
\frac{d}{d t}(|Y(t)|) \lesssim \sqrt{A} \cdot \sqrt{\log A} \cdot|Y(t)|+2^{-\frac{7}{6} A+}+4^{-A}
$$

Integrating in time, we get

$$
\begin{aligned}
\max _{0 \leq t \leq \frac{1}{\log \log A}}|Y(t)| & \lesssim \int_{0}^{\frac{1}{\log \log A}} e^{\frac{1}{\log \log A} \sqrt{A} \cdot \sqrt{\log A}}\left(2^{-\frac{7}{6} A+}+4^{-A}\right) d s \\
& \lesssim 2^{-\frac{7}{6} A+} .
\end{aligned}
$$

Therefore (8.43) is proved.

Step 3. We show that

$$
\begin{aligned}
& \left\|\partial_{r r} u^{r}(t)\right\|_{\infty}+\left\|\partial_{r z} u^{r}(t)\right\|_{\infty}+\left\|\partial_{z z} u^{r}(t)\right\|_{\infty} \\
& \quad+\left\|\partial_{r r} u^{z}(t)\right\|_{\infty}+\left\|\partial_{r z} u^{z}(t)\right\|_{\infty}+\left\|\partial_{z z} u^{z}(t)\right\|_{\infty} \lesssim 2^{A+}, \quad \forall 0 \leq t \leq 1 .
\end{aligned}
$$

and

$$
\begin{aligned}
& \left\|\partial_{r r} U^{r}(t)\right\|_{\infty}+\left\|\partial_{r z} U^{r}(t)\right\|_{\infty}+\left\|\partial_{z z} U^{r}(t)\right\|_{\infty} \\
& \quad+\left\|\partial_{r r} U^{z}(t)\right\|_{\infty}+\left\|\partial_{r z} U^{z}(t)\right\|_{\infty}+\left\|\partial_{z z} U^{z}(t)\right\|_{\infty} \lesssim 2^{A+}, \quad \forall 0 \leq t \leq 1 .
\end{aligned}
$$

We shall only prove (8.49) since the proof for (8.50) is essentially the same.

By a simple energy estimate, we have

$$
\max _{0 \leq t \leq 1}\left(\left\|D^{2} u(t)\right\|_{\infty}+\|D \omega(t)\|_{\infty}\right) \lesssim 2^{A+} .
$$

Write

$$
u=\left(u^{1}, u^{2}, u^{z}\right) .
$$


Obviously

$$
u^{1}\left(t, x_{1}, x_{2}, z\right)=\frac{1}{r} u^{r} x_{1}, \quad u^{2}=\frac{1}{r} u^{r} x_{2} .
$$

Since $u$ is axisymmetric, easy to check that

$$
\left(u^{1}\left(t, x_{1}, x_{2}, z\right), u^{2}\left(t, x_{1}, x_{2}, z\right)\right)=\alpha(t, z)\left(x_{1}, x_{2}\right)+O\left(r^{2}\right),
$$

where $\alpha(t, z)$ is a constant depending only on $(t, z)$. From this and (8.51), it is not difficult to show that

$$
\begin{aligned}
\left|\left(\partial_{2} u^{1}\right)\left(t, 0, x_{2}, z\right)\right| & \lesssim\left\|D^{2} u\right\|_{\infty} \cdot\left|x_{2}\right| \\
& \lesssim 2^{A+}\left|x_{2}\right|, \quad \forall 0 \leq t \leq 1, x_{2} \in \mathbb{R} .
\end{aligned}
$$

By the Fundamental Theorem of Calculus, we then have for any $\left(x_{1}, x_{2}, z\right)(r=$ $\left.\sqrt{x_{1}^{2}+x_{2}^{2}}\right)$

$$
\begin{aligned}
\left|\frac{\left(\partial_{2} u^{1}\right)\left(t, x_{1}, x_{2}, z\right)}{r}\right| & \lesssim\left|\frac{\left(\partial_{2} u^{1}\right)\left(t, x_{1}, x_{2}, z\right)-\left(\partial_{2} u^{1}\right)\left(t, 0, x_{2}, z\right)}{r}\right|+\left|\frac{\left(\partial_{2} u^{1}\right)\left(t, 0, x_{2}, z\right)}{r}\right| \\
& \lesssim 2^{A+} .
\end{aligned}
$$

Denote $g=\frac{1}{r} u^{r}$. Since

$$
u^{1}\left(t, x_{1}, x_{2}, z\right)=g(t, r, z) x_{1}, \quad r=\sqrt{x_{1}^{2}+x_{2}^{2}},
$$

differentiating in $x_{2}$ then gives us

$$
\partial_{2} u^{1}=\left(\partial_{r} g\right) \cdot \frac{x_{2} x_{1}}{r} .
$$

Therefore choosing $\left|x_{1}\right| \sim\left|x_{2}\right| \sim r$ and using (8.52), we obtain

$$
\left\|\partial_{r}\left(\frac{1}{r} u^{r}(t)\right)\right\|_{\infty}=\left\|\partial_{r} g\right\|_{\infty} \lesssim 2^{A+}, \quad \forall 0 \leq t \leq 1 .
$$

By (7.36), we have

$$
\partial_{r r} u^{r}=-\partial_{r}\left(\frac{1}{r} u^{r}\right)-\partial_{z z} u^{r}-\partial_{z} \omega^{\theta} .
$$

By (8.51),

$$
\begin{aligned}
& \left\|\partial_{z z} u^{r}\right\|_{\infty}=\left\|\left(\partial_{z z} u\right) \cdot e_{r}\right\|_{\infty} \lesssim\left\|D^{2} u\right\|_{\infty} \lesssim 2^{A+}, \\
& \left\|\partial_{z} \omega^{\theta}\right\|_{\infty}=\left\|\left(\partial_{z} \omega\right) \cdot e_{\theta}\right\|_{\infty} \lesssim 2^{A+} .
\end{aligned}
$$

Therefore by (8.53) and (8.54), we get

$$
\max _{0 \leq t \leq 1}\left\|\partial_{r r} u^{r}(t)\right\|_{\infty} \lesssim 2^{A+} .
$$

Similar to (8.46), we have

$$
\begin{aligned}
\left|\frac{1}{r}\left(\partial_{z} u^{r}\right)(t, r, z)\right| & =\left|\frac{\left(\left(\partial_{z} u\right)\left(t, x_{1}, x_{2}, z\right)-\left(\partial_{z} u\right)(t, 0,0, z)\right) \cdot e_{r}}{r}\right| \\
& \lesssim\left\|D^{2} u\right\|_{\infty} \lesssim 2^{A+} .
\end{aligned}
$$

Since $\omega^{\theta}=\partial_{r} u^{z}-\partial_{z} u^{r}$, we get

$$
\begin{aligned}
\left\|\frac{1}{r} \partial_{r} u^{z}\right\|_{\infty} & \lesssim\left\|\frac{\omega^{\theta}}{r}\right\|_{\infty}+\left\|\frac{1}{r} \partial_{z} u^{r}\right\|_{\infty} \\
& \lesssim 2^{A+} .
\end{aligned}
$$


We then get

$$
\begin{aligned}
\left\|\partial_{r r} u^{z}\right\|_{\infty} & \lesssim\left\|\Delta u^{z}\right\|_{\infty}+\left\|\frac{1}{r} \partial_{r} u^{z}\right\|_{\infty}+\left\|\partial_{z z} u^{z}\right\|_{\infty} \\
& \lesssim 2^{A+}, \quad \forall 0 \leq t \leq 1 .
\end{aligned}
$$

We have proved that $\left\|\partial_{r r} u^{r}\right\|_{\infty}$ and $\left\|\partial_{r r} u^{z}\right\|_{\infty}$ are both under control. The rest of the terms in (8.49) are similarly estimated. We omit further details.

Step 4. Set $e(t)=(D \phi)(t), E(t)=(D \Phi)(t)$, then obviously

$$
\begin{aligned}
& \partial_{t} E=(D V)(\Phi) E+\left(D v_{1}\right)(\Phi) E+\left(D v_{2}\right)(\Phi) E, \\
& \partial_{t} e=(D v)(\phi) e+\left(D v_{1}\right)(\phi) e .
\end{aligned}
$$

Observe that

$$
D v_{1}=a(t)\left(\begin{array}{rr}
1 & 0 \\
0 & -2
\end{array}\right) .
$$

Set $q=E-e$. By Lemma 8.4 we only need to control $q$ in the region $|x| \lesssim 2^{-A}$. In this region we have

$$
\left\|D v_{2}(\Phi)\right\|_{\infty} \lesssim 2^{-A}
$$

The equation for $q$ takes the form

$$
\begin{aligned}
\partial_{t} q=( & (D V)(\Phi)-(D V)(\phi)) E+((D V)(\phi)-(D v)(\phi)) E \\
& +(D v)(\phi) q+a(t)\left(\begin{array}{rr}
1 & 0 \\
0 & -2
\end{array}\right) q \\
& +\left(D v_{2}\right)(\Phi) E .
\end{aligned}
$$

By (8.48), (8.49), (8.50) and interpolation, we have

$$
\max _{0 \leq t \leq \frac{1}{\log \log A}}\|D(v-V)\|_{\infty} \lesssim 2^{-\frac{1}{12} A+} .
$$

By (8.50), (8.43), (8.39), (8.47) and (8.55), we have

$$
\begin{aligned}
\partial_{t}(|q|) \lesssim & \left\|D^{2} V\right\|_{\infty} \cdot|\phi-\Phi| \cdot\|E\|_{\infty}+\|D(V-v)\|_{\infty} \cdot\|E\|_{\infty} \\
& +\|D v\|_{\infty} \cdot|q|+B \cdot|q|+\left\|D v_{2}(\Phi)\right\|_{\infty} \cdot\|E\|_{\infty} \\
\lesssim & 2^{A+} \cdot 2^{-\frac{7}{6} A+} \cdot(\log \log \log A)^{2} \\
& +2^{-\frac{1}{12} A+} \cdot(\log \log \log A)^{2}+(\sqrt{A} \cdot \sqrt{\log A}+B)|q| \\
& +2^{-A} \cdot(\log \log \log A)^{2} .
\end{aligned}
$$

Integrating in time $t \leq \frac{1}{\log \log A}$, we then obtain

$$
\max _{0 \leq t \leq \frac{1}{\log \log A}}\|q(t)\|_{\infty} \lesssim 1 .
$$

But this obviously contradicts (8.25).

The next proposition is the key to our construction in the 3D compactly supported data case. It is written in the same style as in Lemma 6.4. The overall statement of the proposition is a bit long and over-stretched due to some additional technical conditions pertaining to the 3D situation. Nevertheless the structure of the proposition is the same as that in Lemma 6.4. In short summary the main body of the proposition should read as " Let $\omega_{-1}$ satisfy ...Then for any $0<\epsilon<\epsilon_{0}$, one 
can find $\omega_{0}$ with the properties $\ldots$ and $\delta_{0}$ such that for any $\omega_{j}$ with the properties ..., the following hold true: ....".

Proposition 8.6. Let $\omega_{-1} \in C_{c}^{\infty}(B(0,100))$ be a given axisymmetric function such that $\omega_{-1}=\omega_{-1}^{\theta} e_{\theta}, \omega_{-1}^{\theta}=\omega_{-1}^{\theta}(r, z)$ is scalar-valued and for some $r_{-1}>0$, $0<R_{0}<\frac{1}{100}$,

$$
\operatorname{supp}\left(\omega_{-1}^{\theta}\right) \subset\left\{(r, z): r>r_{-1}, z \leq-4 R_{0}\right\} .
$$

Denote $u_{-1}=-\Delta^{-1} \nabla \times \omega_{-1}$ and

$$
u_{-1}^{*}=\left\|u_{-1}\right\|_{2} .
$$

Then for any $0<\epsilon \leq \epsilon_{0}$ with $\epsilon_{0}=\epsilon_{0}\left(\omega_{-1}\right) \ll R_{0}$ sufficiently small, we can find a smooth axisymmetric function $\omega_{0}=\omega_{0}^{\theta} e_{\theta}$ (depending only on $\left(\epsilon, \omega_{-1}\right)$ ) with the properties:

- $\omega_{0} \in C_{c}^{\infty}(B(0,100))$ and for some $r_{0}>0$,

$$
\operatorname{supp}\left(\omega_{0}^{\theta}(r, z)\right) \subset\left\{(r, z): r_{0}<r<\epsilon,-\epsilon<z<\epsilon\right\} .
$$

- $\left\|\omega_{0}\right\|_{\infty} \exp \left(C\left\|\frac{\omega_{0}}{r}\right\|_{L^{3,1}}\right)<\delta\left(\epsilon^{2}, \omega_{-1}\right)$ (see (8.3) $)$;

- denote $u_{0}=-\Delta^{-1} \nabla \times \omega_{0}$, then

$$
\left\|u_{0}\right\|_{2}<\epsilon u_{-1}^{*}<\frac{1}{4} u_{-1}^{*}
$$

and $\delta_{0}=\delta_{0}\left(\omega_{-1}, \omega_{0}\right) \ll \epsilon$ sufficiently small such that for any smooth axisymmetric functions $\omega_{j}=\omega_{j}^{\theta} e_{\theta}, 1 \leq j \leq N$ (here $N \geq 1$ is arbitrary but finite) with the properties:

- $\omega_{j} \in C_{c}^{\infty}(B(0,100))$ and $\operatorname{supp}\left(\omega_{j}^{\theta}\right) \subset\left\{(r, z): r>r_{j}, z>2 R_{0}\right\}$ for some $r_{j}>0$.

- for each $j \geq 1$, denote $f_{j}=\omega_{-1}^{\theta}+\omega_{0}^{\theta}+\sum_{i=1}^{j-1} \omega_{i}^{\theta}$, then

$$
\left\|\omega_{j}^{\theta}\right\|_{\infty} \cdot \exp \left(C\left\|\frac{\omega_{j}^{\theta}}{r}\right\|_{L^{3,1}}\right)<\delta_{j}
$$

where $\delta_{j}=\delta\left(2^{-3 j} \delta_{0}, f_{j}\right)$ as defined in (8.3);

- denote $u_{j}=-\Delta^{-1} \nabla \times \omega_{j}$, then

$$
\left\|u_{j}\right\|_{2}<\frac{\epsilon}{2^{j+1}} u_{-1}^{*}
$$

the following hold true:

Let $\omega$ be the smooth solution to the axisymmetric system

$$
\left\{\begin{array}{l}
\partial_{t}\left(\frac{\omega}{r}\right)+(u \cdot \nabla)\left(\frac{\omega}{r}\right)=0, \quad 0<t \leq 1, \\
u=-\Delta^{-1} \nabla \times \omega, \\
\left.\omega\right|_{t=0}=\left(\omega_{-1}^{\theta}+\omega_{0}^{\theta}+\sum_{j=1}^{N} \omega_{j}^{\theta}\right) e_{\theta},
\end{array}\right.
$$

then

(1) for any $0 \leq t \leq \epsilon$, we have the decomposition

$$
\omega(t)=\omega_{A}(t)+\omega_{B}(t)+\omega_{C}(t)
$$


where

$$
\begin{aligned}
& \operatorname{supp}\left(\omega_{A}(t)\right) \subset\left\{(r, z): z \leq-4 R_{0}+\sqrt{\epsilon}\right\} \\
& \operatorname{supp}\left(\omega_{B}(t)\right) \subset\{(r, z):|z| \leq \sqrt{\epsilon}\} \\
& \operatorname{supp}\left(\omega_{C}(t)\right) \subset\left\{(r, z): z \geq 2 R_{0}-\sqrt{\epsilon}\right\}
\end{aligned}
$$

and $\omega_{A}(t=0)=\omega_{-1}, \omega_{B}(t=0)=\omega_{0}^{\theta} e_{\theta}, \omega_{C}(t=0)=\left(\sum_{j=1}^{N} \omega_{j}^{\theta}\right) e_{\theta}$.

(2) the $L^{\infty}$ norm of $\omega_{B}$ and $\omega_{C}$ is uniformly small on the interval $[0,1]$ :

$$
\max _{0 \leq t \leq 1}\left(\left\|\omega_{B}(t)\right\|_{L^{\infty}}+\left\|\omega_{C}(t)\right\|_{L^{\infty}}\right) \leq \epsilon
$$

(3) the $\dot{H}^{\frac{3}{2}}-$ norm of $\omega_{B}$ is inflated rapidly on the time interval $[0, \epsilon]$ : there exists $0<t_{0}^{1}=t_{0}^{1}\left(\epsilon, \omega_{-1}, \omega_{0}\right)<\epsilon, 0<t_{0}^{2}=t_{0}^{2}\left(\epsilon, \omega_{-1}, \omega_{0}\right)<\epsilon$, such that

$$
\begin{aligned}
& \left\|\omega_{B}(t=0)\right\|_{\dot{H}^{\frac{3}{2}}}<\epsilon, \\
& \left\|\omega_{B}(t)\right\|_{\dot{H}^{\frac{3}{2}}}>\frac{1}{\epsilon}, \quad \text { for any } t_{0}^{1} \leq t \leq t_{0}^{2} .
\end{aligned}
$$

(4) all $H^{k}, k \geq 2$ norms of $\omega_{B}$ can be bounded purely in terms of initial data $\omega_{0}$ on the time interval $[0, \epsilon]$ : for any $k \geq 2$,

$$
\max _{0 \leq t \leq \epsilon}\left\|\omega_{B}(t)\right\|_{H^{k}} \leq C\left(k, R_{0}, u_{-1}^{*}\right)\left\|\omega_{0}\right\|_{H^{k}}
$$

Note here the bound of $\left\|\omega_{B}\right\|_{H^{k}}$ is "almost local" in the sense that it depends only on $u_{-1}^{*}$ but not on other higher Sobolev norms of $\omega_{A}$ or $\omega_{C}$. Similarly we have

$$
\max _{0 \leq t \leq \epsilon}\left\|\omega_{A}(t)\right\|_{H^{k}} \leq C\left(k, R_{0}, u_{-1}^{*}\right)\left\|\omega_{-1}\right\|_{H^{k}}, \quad \forall k \geq 2 .
$$

Proof of Proposition 8.6. The nontrivial point is to find $\omega_{0}$ such that (8.60) is achieved. We first show that a generic $\omega_{0}$ (i.e. satisfying the properties specified in (8.56) (8.57) ) is enough to make (8.58), (8.59) and (8.61) hold.

By conservation of $\|u(t)\|_{2}$, we have

$$
\begin{aligned}
\|u(t)\|_{2}=\|u(0)\|_{2} & \leq u_{-1}^{*}+\sum_{j=1}^{\infty} u_{-1}^{*} \cdot 2^{-j} \\
& \leq 2 u_{-1}^{*}, \quad \forall t \geq 0 .
\end{aligned}
$$

Let $\omega_{L}$ be the smooth solution to the axisymmetric system

$$
\left\{\begin{array}{l}
\partial_{t}\left(\frac{\omega_{L}}{r}\right)+\left(u_{L} \cdot \nabla\right)\left(\frac{\omega_{L}}{r}\right)=0, \quad 0<t \leq 1 \\
u_{L}=-\Delta^{-1} \nabla \times \omega_{L} \\
\omega_{L}(t=0)=\omega_{-1}
\end{array}\right.
$$

Obviously

$$
\max _{0 \leq t \leq 1}\left\|\omega_{L}(t)\right\|_{\infty} \lesssim \omega_{-1} 1 .
$$

By Lemma 8.1 we have

$$
\max _{0 \leq t \leq 1}\left\|\omega(t)-\omega_{L}(t)\right\|_{\infty} \ll \epsilon
$$

and clearly

$$
\max _{0 \leq t \leq 1}\|\omega(t)\|_{\infty} \lesssim \omega_{-1} 1 .
$$


Interpolating the above with (8.63) then gives

$$
\max _{0 \leq t \leq 1}\|u(t)\|_{\infty} \leq c_{1},
$$

where $c_{1}>0$ is a constant depending only on $\omega_{-1}$.

This shows that the support of $\omega(t)$ moves at a speed at most $c_{1}$. Since we can always choose $\epsilon$ sufficiently small such that $c_{1} \epsilon \ll \sqrt{\epsilon}$, the decomposition (8.58) then obviously follows.

The inequality (8.59) is a simple consequence of Lemma 8.1] To show (8.61), we note that for $0 \leq t \leq \epsilon, \omega_{B}=\omega_{B}(t)$ solves the equation

$$
\partial_{t} \omega_{B}+\left(\left(u_{B}+u_{e x}\right) \cdot \nabla\right) \omega_{B}=\left(\omega_{B} \cdot \nabla\right)\left(u_{B}+u_{e x}\right)
$$

where

$$
\begin{aligned}
& u_{B}(t)=-\Delta^{-1} \nabla \times \omega_{B}(t), \\
& u_{e x}(t)=-\Delta^{-1} \nabla \times\left(\omega_{A}(t)+\omega_{C}(t)\right) .
\end{aligned}
$$

Since for $0 \leq t \leq \epsilon$ and $\epsilon$ sufficiently small,

$$
\begin{aligned}
& d\left(\operatorname{supp}\left(\omega_{A}(t)\right), \operatorname{supp}\left(\omega_{B}(t)\right)\right) \geq R_{0}, \\
& d\left(\operatorname{supp}\left(\omega_{C}(t)\right), \operatorname{supp}\left(\omega_{B}(t)\right)\right) \geq R_{0},
\end{aligned}
$$

we can then write for $x \in \operatorname{supp}\left(\omega_{B}(t)\right)$,

$$
u_{e x}(t, x)=\int_{R^{3}} K(x-y)\left(\omega_{A}(t, y)+\omega_{C}(t, y)\right) d y,
$$

where the modified kernel $K(\cdot)$ satisfies

$$
\left|\left(\partial^{\alpha} K\right)(x)\right| \lesssim R_{0}, \alpha\left(1+|x|^{2}\right)^{-\frac{2+|\alpha|}{2}}, \quad \forall x \in \mathbb{R}^{3},|\alpha| \geq 0 .
$$

Since $\omega(t)=\nabla \times u(t)$, we can rewrite (18.64) as

$$
\begin{aligned}
u_{e x}(t, x) & =\int_{\mathbb{R}^{3}} K(x-y) \omega(t, y) d y-\int_{\mathbb{R}^{3}} K(x-y) \omega_{B}(t, y) d y \\
& =\int_{\mathbb{R}^{3}} K(x-y) \nabla \times u(t, y) d y-\int_{\mathbb{R}^{3}} K(x-y) \omega_{B}(t, y) d y \\
& =\int_{\mathbb{R}^{3}} \tilde{K}(x-y) u(t, y) d y-\int_{\mathbb{R}^{3}} K(x-y) \omega_{B}(t, y) d y \\
& =: u_{e x}^{(1)}(t, x)+u_{e x}^{(2)}(t, x) .
\end{aligned}
$$

Obviously we only need to bound $u_{e x}^{(1)}$. Since $\left|\left(\partial^{\alpha} \tilde{K}\right)(x)\right| \lesssim R_{0}, \alpha\left(1+|x|^{2}\right)^{-\frac{3+|\alpha|}{2}}$, we have

$$
\left\|u_{e x}^{(1)}(t, \cdot)\right\|_{H^{k}} \lesssim_{k, R_{0}}\|u(t)\|_{2} \lesssim_{k, R_{0}} u_{-1}^{*}, \quad \text { for any } k \geq 0 .
$$

The inequality (8.61) then easily follows from this and a simple energy estimate. Similarly one can prove (8.62).

It remains for us to show the existence of $\omega_{0}$ such that (8.60) hold. First we show that it suffices to consider the following reduced system

$$
\left\{\begin{array}{l}
\partial_{t}\left(\frac{W}{r}\right)+(U \cdot \nabla)\left(\frac{W}{r}\right)=0, \quad 0<t \leq 1, \\
U=-\Delta^{-1} \nabla \times W, \\
\left.W\right|_{t=0}=\omega_{-1}+\omega_{0} .
\end{array}\right.
$$


By Lemma 8.1 and our assumptions on $\omega_{j}$, we have

$$
\max _{0 \leq t \leq 1}\|\omega(t)-W(t)\|_{\infty} \lesssim \delta_{0} \ll \epsilon .
$$

Since $\max _{0 \leq t \leq 1}\|u(t)\|_{\infty} \lesssim \omega_{-1} 1$ and $\max _{0 \leq t \leq 1}\|U(t)\|_{\infty} \lesssim \omega_{-1} 1$, we have for some $c_{2}=c_{2}\left(\omega_{-1}\right)>0$,

$$
\begin{aligned}
& \operatorname{supp}(W(t)) \subset B\left(0,100+c_{2}\right), \quad \forall 0 \leq t \leq 1, \\
& \operatorname{supp}(\omega(t)) \subset B\left(0,100+c_{2}\right), \quad \forall 0 \leq t \leq 1 .
\end{aligned}
$$

Therefore by (8.65) and Hölder, we get

$$
\max _{0 \leq t \leq 1}\|\omega(t)-W(t)\|_{2} \lesssim \omega_{-1} \delta_{0}^{\frac{1}{2}} .
$$

For $0 \leq t \leq \epsilon$, we write the decomposition of $W$ as

$$
W(t)=W_{L}(t)+W_{R}(t),
$$

where

$$
\begin{aligned}
& \operatorname{supp}\left(W_{L}(t)\right) \subset\left\{(r, z): z \leq-4 R_{0}+\sqrt{\epsilon}\right\} \\
& \operatorname{supp}\left(W_{R}(t)\right) \subset\{(r, z):|z| \leq \sqrt{\epsilon}\}
\end{aligned}
$$

and $W_{L}(t=0)=\omega_{-1}, W_{R}(t=0)=\omega_{0}$.

By (8.61) and a similar bound for $W_{R}(t)$, we have

$$
\max _{0 \leq t \leq \epsilon}\left\|\omega_{B}(t)-W_{R}(t)\right\|_{H^{3}} \lesssim \omega_{-1}, R_{0}, u_{-1}^{*}\left\|\omega_{0}\right\|_{H^{3}} .
$$

Interpolating the above with (8.66) and choosing $\delta_{0}$ sufficiently small, we then get

$$
\max _{0 \leq t \leq \epsilon}\left\|\omega_{B}(t)-W_{R}(t)\right\|_{H^{2}} \leq \epsilon .
$$

This shows that it suffices for us to inflate the $\left\|W_{R}(t)\right\|_{\dot{H}^{\frac{3}{2}}}$ norm.

To this end, let $W^{1}$ be the smooth solution to

$$
\left\{\begin{array}{l}
\partial_{t}\left(\frac{W^{1}}{r}\right)+\left(U^{1} \cdot \nabla\right)\left(\frac{W^{1}}{r}\right)=0, \quad 0<t \leq 1, \\
U^{1}=-\Delta^{-1} \nabla \times W^{1}, \\
\left.W^{1}\right|_{t=0}=\omega_{-1}+\tilde{g}_{A} e_{\theta},
\end{array}\right.
$$

where $\tilde{g}_{A}$ is the same as defined in (8.21) and we shall take $A$ to be sufficiently large without too much explicit mentioning. Eventually we shall take $\omega_{0}$ to be a suitable perturbation of $\tilde{g}_{A}$ and let $W$ be the corresponding solution.

For $0 \leq t \leq \frac{1}{\log \log A}$, we can decompose the solution $W^{1}$ as

$$
W^{1}(t)=W_{L}^{1}(t)+W_{R}^{1}(t)
$$

where

$$
\begin{array}{r}
\operatorname{supp}\left(W_{L}^{1}(t)\right) \subset\left\{(r, z): z \leq-4 R_{0}+\frac{1}{\sqrt{\log \log A}}\right\}, \\
\operatorname{supp}\left(W_{R}^{1}(t)\right) \subset\left\{(r, z):|z| \leq \frac{1}{\sqrt{\log \log A}}\right\} .
\end{array}
$$


The equation for $W_{R}^{1}$ takes the form

$$
\left\{\begin{array}{l}
\partial_{t}\left(\frac{W_{R}^{1}}{r}\right)+\left(\left(U_{R}^{1}+U_{L}^{1}\right) \cdot \nabla\right)\left(\frac{W_{R}^{1}}{r}\right)=0 \\
U_{R}^{1}=-\Delta^{-1} \nabla \times W_{R}^{1} \\
U_{L}^{1}=-\Delta^{-1} \nabla \times W_{L}^{1} \\
W_{R}^{1}(t=0)=\tilde{g}_{A} e_{\theta}
\end{array}\right.
$$

Write

$$
U_{L}^{1}=U_{L}^{r} e_{r}+U_{L}^{z} e_{z}
$$

Let $\xi(t)$ solves the ODE

$$
\left\{\begin{array}{l}
\frac{d}{d t} \xi(t)=-U_{L}^{z}(0,0, \xi(t)), \\
\xi(0)=0 .
\end{array}\right.
$$

We can expand $U_{L}^{1}(t)$ near the point $(0,0, \xi(t))$ to get

$$
U_{L}^{1}\left(t, x_{1}, x_{2}, z+\xi(t)\right)=U_{L}^{z}(t, 0,0, \xi(t)) e_{z}+\underbrace{a(t) r e_{r}-2 a(t) z e_{z}}_{=: u_{1}\left(t, x_{1}, x_{2}, z\right)}+u_{2}\left(t, x_{1}, x_{2}, z\right),
$$

where for any $0 \leq t \leq \frac{1}{\log \log A}$,

$$
\begin{aligned}
& |a(t)| \lesssim_{\omega_{-1}, R_{0}} 1, \\
& \left|u_{2}(t, x)\right| \lesssim_{\omega_{-1}, R_{0}}|x|^{2}, \\
& \left|\left(D u_{2}\right)(t, x)\right| \lesssim_{\omega_{-1}, R_{0}}|x|, \\
& \left|\left(D^{2} u_{2}\right)(t, x)\right| \lesssim_{\omega_{-1}, R_{0}} 1, \quad \forall x \in \mathbb{R}^{3} .
\end{aligned}
$$

Note that $u_{2}$ is axisymmetric without swirl, i.e. $u_{2}=u_{2}^{r} e_{r}+u_{2}^{z} e_{z}$.

Introduce $\Omega(t)=\Omega\left(t, x_{1}, x_{2}, z\right)$ such that

$$
\begin{aligned}
& \Omega\left(t, x_{1}, x_{2}, z\right):=W_{R}^{1}\left(t, x_{1}, x_{2}, z+\xi(t)\right), \\
& U_{\Omega}(t):=-\Delta^{-1} \nabla \times \Omega(t) .
\end{aligned}
$$

It is then not difficult to check that the equation for $\Omega$ takes the form

$$
\partial_{t}\left(\frac{\Omega}{r}\right)+\left(\left(U_{\Omega}+u_{1}+u_{2}\right) \cdot \nabla\right)\left(\frac{\Omega}{r}\right)=0 .
$$

Let $\Phi_{\Omega}=\left(\Phi_{\Omega}^{r}, \Phi_{\Omega}^{z}\right)$ be the characteristic line associated with $U_{\Omega}+u_{1}+u_{2}$ and let $\tilde{\Phi}_{\Omega}$ be the corresponding inverse map. By Lemma 8.5 for $A$ sufficiently large, we have either

or

$$
\max _{0 \leq t \leq \frac{1}{\log \log A}}\|\Omega(t)\|_{\dot{H}^{\frac{3}{2}}}>\log \log \log A,
$$

$$
\max _{0 \leq t \leq \frac{1}{\log \log A}}\left\|\left(D \tilde{\Phi}_{\Omega}\right)(t)\right\|_{\infty}>\log \log \log A
$$

must hold.

Now discuss two cases.

Case 1: (8.67) hold. In this case easy to check that

$$
\max _{0 \leq t \leq \frac{1}{\log \log A}}\left\|W_{R}^{1}(t)\right\|_{\dot{H}^{\frac{3}{2}}} \gtrsim \log \log \log A .
$$

Therefore we can just let $W(t)=W^{1}(t)$ with $\omega_{0}=\tilde{g}_{A}$. 
Case 2: 8.68) hold. In this case we just need to apply a perturbation argument similar to that in the proof of Proposition 7.11. Easy to check that this case is also OK.

Concluding from the above two cases, the proposition is proved.

We are now ready to complete the

Proof of Theorem 1.10. We shall only sketch the proof for $\omega_{0}^{(g)} \equiv 0$. The construction of $\omega_{0}^{(p)}$ for the general nonzero $\omega_{0}^{(g)}$ is a simple modification of the proof presented below. For example, one can just take the first patch as $\omega_{0}^{(g)}$ and start the perturbation for $j \geq 2$.

We now begin the proof for $\omega_{0}^{(g)} \equiv 0$. For each integer $j \geq 1$, define $x_{*}^{j}=$ $\left(0,0, \sum_{k=1}^{j} \frac{1}{2^{k}}\right)$. Obviously for any $j \geq 2$, we have

$$
\begin{aligned}
& \left|x_{*}^{j+1}-x_{*}^{j}\right|=\frac{1}{2^{j+1}}, \\
& \left|x_{*}^{j}-x_{*}^{j-1}\right|=\frac{1}{2^{j}} .
\end{aligned}
$$

We shall choose $x_{*}^{j}$ to be the center of the $j^{t h}$ patch. So the distance between the nearest patches is about $2^{-j}$. Define

$$
x_{*}=\lim _{j \rightarrow \infty} x_{*}^{j}=(0,0,1) .
$$

Our constructed solution will exhibit some additional regularity away from the limit point $x_{*}$. form)

Let $W^{1}$ be a smooth axisymmetric solution to the Euler equation (in vorticity

$$
\left\{\begin{array}{l}
\partial_{t}\left(\frac{W^{1}}{r}\right)+\left(U^{1} \cdot \nabla\right)\left(\frac{W^{1}}{r}\right)=0, \quad 0<t \leq 1, x=\left(x_{1}, x_{2}, z\right), r=\sqrt{x_{1}^{2}+x_{2}^{2}} \\
U^{1}=-\Delta^{-1} \nabla \times W^{1} \\
\left.W^{1}\right|_{t=0}=W_{0}^{1}=W_{0}^{1, \theta} e_{\theta}
\end{array}\right.
$$

such that $W_{0}^{1, \theta}=W_{0}^{1, \theta}(r, z)$ is scalar-valued, $\operatorname{supp}\left(W_{0}^{1, \theta}\right) \subset\left\{(r, z): r>r_{0}\right\}$ for some $r_{0}>0, W^{1}(t) \in C_{c}^{\infty}\left(B\left(x_{*}^{1}, \frac{1}{2^{10}}\right)\right)$ for any $0 \leq t \leq 1$ and

$$
\left\|U^{1}(0, \cdot)\right\|_{H^{\frac{5}{2}}}+\max _{0 \leq t \leq 1}\left\|W^{1}(t, \cdot)\right\|_{\infty} \leq \frac{1}{2^{100}}
$$

In view of the scaling symmetry $\left(\omega \rightarrow \omega_{\lambda}(t, x)=\lambda \omega(\lambda t, x)\right)$ and translation symmetry (in the axisymmetric case we just shift only along the $z$-axis so as to keep axisymmetry) of the Euler equation, we can always find a nonzero $W^{1}$ satisfying the aforementioned conditions by transforming an arbitrary compactly supported solution.

By repeated applying Proposition 8.6 (one needs to shift along the $z$-axis if necessary), we can find a sequence of smooth solutions $W^{j}, j \geq 2$, solving the equations

$$
\left\{\begin{array}{l}
\partial_{t}\left(\frac{W^{j}}{r}\right)+\left(U^{j} \cdot \nabla\right)\left(\frac{W^{j}}{r}\right)=0, \quad 0<t \leq 1 \\
U^{j}=-\Delta^{-1} \nabla \times W^{j} \\
\left.W^{j}\right|_{t=0}=W_{0}^{j}=W_{0}^{j, \theta} e_{\theta}
\end{array}\right.
$$

such that the following hold: 
- $W_{0}^{j}=\left(\sum_{k=1}^{j} f_{k}\right) e_{\theta}$, where $f_{1}=W_{0}^{1, \theta}$, and for $k \geq 2, \operatorname{supp}\left(f_{k}\right) \subset\{(r, z)$ : $\left.r>r_{k}\right\}$ for some $r_{k}>0$.

- Define $F_{k}=f_{k} e_{\theta}$. Then for each $k \geq 1, F_{k} \in C_{c}^{\infty}\left(B\left(x_{*}^{k}, \frac{1}{2^{10 k}}\right)\right)$. Furthermore

$$
\left\|\Delta^{-1} \nabla \times F_{k}\right\|_{H^{\frac{5}{2}}} \leq 2^{-100 k}, \quad \forall k \geq 1 .
$$

- For any $j \geq 2$,

$$
\max _{0 \leq t \leq 1}\left\|W^{j}(t, \cdot)-W^{j-1}(t, \cdot)\right\|_{\infty} \leq 2^{-100 j} .
$$

- For each $j_{0} \geq 2$, there exists $t_{j_{0}}^{1}, t_{j_{0}}^{2}$ with $0<t_{j_{0}}^{1}<t_{j_{0}}^{2}<2^{-j_{0}}$, such that for any $j \geq j_{0}+2$, we have the decomposition:

$$
W^{j}(t, x)=W_{<j_{0}}^{j}(t, x)+W_{j_{0}}^{j}(t, x)+W_{>j_{0}}^{j}(t, x), \quad \forall t \leq t_{j_{0}}^{2},
$$

where $W_{<j_{0}}^{j} \in C_{c}^{\infty}\left(\mathbb{R}^{3}\right), W_{j_{0}}^{j} \in C_{c}^{\infty}\left(\mathbb{R}^{3}\right), W_{>j_{0}}^{j} \in C_{c}^{\infty}\left(\mathbb{R}^{3}\right)$ satisfy

$$
\begin{aligned}
& \operatorname{supp}\left(W_{<j_{0}}^{j}\right) \subset\left\{x=\left(x_{1}, x_{2}, z\right): z \leq \sum_{k=1}^{j_{0}-1} 2^{-k}+\frac{1}{8} \cdot 2^{-j_{0}}\right\} \\
& \operatorname{supp}\left(W_{j_{0}}^{j}\right) \subset\left\{x=\left(x_{1}, x_{2}, z\right): \sum_{k=1}^{j_{0}} 2^{-k}-\frac{1}{8} \cdot 2^{-j_{0}}<z<\sum_{k=1}^{j_{0}} 2^{-k}+\frac{1}{8} \cdot 2^{-j_{0}}\right\} \\
& \operatorname{supp}\left(W_{>j_{0}}^{j}\right) \subset\left\{x=\left(x_{1}, x_{2}, z\right): z>\sum_{k=1}^{j_{0}} 2^{-k}+\frac{1}{4} 2^{-j_{0}}\right\}
\end{aligned}
$$

Here

$$
\begin{aligned}
& W_{<j_{0}}^{j}(t=0)=\sum_{k=1}^{j_{0}-1} F_{k}, \quad W_{j_{0}}^{j}(t=0)=F_{j_{0}}, \\
& W_{>j_{0}}^{j}(t=0)=\sum_{k=j_{0}+1}^{j} F_{k} .
\end{aligned}
$$

Furthermore

$$
\begin{aligned}
& \left\|W_{j_{0}}^{j}(t, \cdot)\right\|_{\dot{H}^{\frac{3}{2}\left(\mathbb{R}^{3}\right)}}>j_{0}, \quad \forall t \in\left[t_{j_{0}}^{1}, t_{j_{0}}^{2}\right] ; \\
& \left\|W_{j_{0}}^{j}(t, \cdot)\right\|_{L^{2}\left(\mathbb{R}^{3}\right)} \leq 2^{-100 j_{0}}, \quad \forall t \leq t_{j_{0}}^{2} \cdot \\
& \max _{0 \leq t \leq t_{j_{0}}^{2}}\left(\left\|W_{j_{0}}^{j}(t, \cdot)\right\|_{H^{k}\left(\mathbb{R}^{3}\right)}+\left\|W_{<j_{0}}^{j}(t, \cdot)\right\|_{H^{k}\left(\mathbb{R}^{3}\right)}\right) \leq C_{j_{0}, k}<\infty, \quad \forall k \geq 2,
\end{aligned}
$$

where $C_{j_{0}, k}$ is a constant depending only on $k$ and $\left(F_{1}, F_{2}, \cdots, F_{j_{0}}\right)$.

We now show the existence of the solution $\omega$ as the limit of $W^{j}, j \rightarrow \infty$. By $L^{2}$-conservation of velocity and (8.70), we have

$$
\begin{aligned}
\max _{0 \leq t \leq 1}\left\|U^{j}(t, \cdot)\right\|_{2} & =\left\|U^{j}(0, \cdot)\right\|_{2} \\
& \leq \sum_{k=1}^{\infty} 2^{-100 k} \leq 2^{-99}, \quad \forall j \geq 1 .
\end{aligned}
$$


By (8.69) and (8.71),

$$
\max _{0 \leq t \leq 1}\left\|W^{j}(t, \cdot)\right\|_{\infty} \leq \sum_{k=1}^{j} 2^{-100 k} \leq 2^{-99}, \quad \forall j \geq 1 .
$$

By (8.76), (8.77) and interpolation, we then get

$$
\sup _{j \geq 1} \max _{0 \leq t \leq 1}\left\|U^{j}(t, \cdot)\right\|_{\infty} \lesssim 1 .
$$

Since $\operatorname{supp}\left(W^{j}(t, \cdot)\right) \subset B(0,2)$, (8.78) then implies that

$$
\operatorname{supp}\left(W^{j}(t, \cdot)\right) \subset B\left(0, C_{1}\right), \quad \forall 0 \leq t \leq 1, j \geq 1,
$$

where $C_{1}>0$ is an absolute constant. By (8.71) and (8.79), the sequence $W^{j}$ is Cauchy in the space $C_{t}^{0} C_{x}^{0}\left([0,1] \times \overline{B\left(0, C_{1}\right)}\right)$ and hence converges to the limit solution $w$ in the same space. By Sobolev embedding and interpolation, it is not difficult to check that $U^{j}$ also converges to $u=-\Delta^{-1} \nabla \times \omega \in C_{t}^{0} L_{x}^{2} \cap C_{t}^{0} C_{x}^{\alpha}\left([0,1] \times \mathbb{R}^{3}\right)$ for any $0<\alpha<1$. It follows easily that $\omega$ is the desired solution satisfying the first two statements in Theorem 1.10

It remains for us to check the last two properties of $\omega$ in Theorem 1.10.

Fix any $j_{0} \geq 2$. By (8.72), (8.75) and taking the limit $j \rightarrow \infty$, we get the decomposition of $\omega(t, x)$ for $t \leq t_{j_{0}}^{2}$ as

$$
\omega(t, x)=\omega_{<j_{0}}(t, x)+\omega_{j_{0}}(t, x)+\omega_{>j_{0}}(t, x),
$$

where $\omega_{<j_{0}}(t) \in C_{c}^{\infty}\left(\mathbb{R}^{3}\right), \omega_{j_{0}}(t) \in C_{c}^{\infty}\left(\mathbb{R}^{3}\right), \omega_{>j_{0}}(t) \in C_{c}^{0}\left(\mathbb{R}^{3}\right)$ for $t \leq t_{j_{0}}^{2}$, and

$$
\begin{aligned}
& \operatorname{supp}\left(\omega_{<j_{0}}\right) \subset\left\{x=\left(x_{1}, x_{2}, z\right): z \leq \sum_{k=1}^{j_{0}-1} 2^{-k}+\frac{1}{8} \cdot 2^{-j_{0}}\right\} \\
& \operatorname{supp}\left(\omega_{j_{0}}\right) \subset\left\{x=\left(x_{1}, x_{2}, z\right): \sum_{k=1}^{j_{0}} 2^{-k}-\frac{1}{8} \cdot 2^{-j_{0}}<z<\sum_{k=1}^{j_{0}} 2^{-k}+\frac{1}{8} \cdot 2^{-j_{0}}\right\} \\
& \operatorname{supp}\left(\omega_{>j_{0}}\right) \subset\left\{x=\left(x_{1}, x_{2}, z\right): z>\sum_{k=1}^{j_{0}} 2^{-k}+\frac{1}{4} 2^{-j_{0}}\right\} .
\end{aligned}
$$

Furthermore

$$
\begin{aligned}
& \left\|\omega_{j_{0}}(t, \cdot)\right\|_{\dot{H}^{\frac{3}{2}\left(\mathbb{R}^{3}\right)}} \geq j_{0}, \quad \forall t \in\left[t_{j_{0}}^{1}, t_{j_{0}}^{2}\right] ; \\
& \left\|\omega_{j_{0}}(t, \cdot)\right\|_{L^{2}\left(\mathbb{R}^{3}\right)} \leq 2^{-100 j_{0}}, \quad \forall t \leq t_{j_{0}}^{2} \cdot \\
& \max _{0 \leq t \leq t_{j_{0}}^{2}}\left(\left\|\omega_{j_{0}}(t, \cdot)\right\|_{H^{k}\left(\mathbb{R}^{3}\right)}+\left\|\omega_{<j_{0}}(t, \cdot)\right\|_{H^{k}\left(\mathbb{R}^{3}\right)}\right) \leq C_{j_{0}, k}<\infty, \quad \forall k \geq 2,
\end{aligned}
$$

where $C_{j_{0}, k}$ is a constant depending only on $k$ and $\left(F_{1}, F_{2}, \cdots, F_{j_{0}}\right)$. Now for any $y=\left(y_{1}, y_{2}, y_{3}\right) \neq x_{*}=(0,0,1)$, consider three cases. If $y_{3} \geq 1$, then in this case by our choice of initial data and finite transport speed, we can find a small neighborhood $N_{y}$ of $y$ and $0<t_{y}<1$ such that $\omega(t, x)=0$ for any $x \in N_{y}$ and $0 \leq t \leq t_{y}$. If $y_{3}<1$, then we can choose $j_{0}$ sufficiently large such that $y \in\left\{x=\left(x_{1}, x_{2}, z\right): z<\sum_{k=1}^{j_{0}-1} 2^{-k}+\frac{1}{16} \cdot 2^{-j_{0}}\right\}$. In this case we can just choose $t_{y}=t_{j_{0}}^{2}$ and $N_{y}$ to be a small open neighborhood contained in $\left\{x=\left(x_{1}, x_{2}, z\right)\right.$ : $\left.z<\sum_{k=1}^{j_{0}-1} 2^{-k}+\frac{1}{16} \cdot 2^{-j_{0}}\right\}$. By (8.83) $\omega(t) \in C^{\infty}\left(N_{y}\right)$ for any $0 \leq t \leq t_{y}$. Therefore statement (3) in Theorem 1.10 is proved. 
Finally we prove statement (4) in Theorem 1.10. For each integer $n \geq 1$, we shall take $j_{n}$ to be sufficiently large and decompose $\omega$ according to (8.80) with $j_{0}$ replaced by $j_{n}$. By a slight abuse of notation we denote $t_{n}^{1}=t_{j_{n}}^{1}, t_{n}^{2}=t_{j_{n}}^{2}$ and $\omega_{n}=\omega_{j_{n}}$. Define

$$
\begin{aligned}
& K_{n}=\overline{\left\{x \in \mathbb{R}^{3}: \omega_{n}(t, x) \neq 0 \text { for some } 0 \leq t \leq t_{n}^{2}\right\}}, \\
& \Omega_{n}^{1}=\left\{x \in \mathbb{R}^{3}: \operatorname{dist}\left(x, K_{n}\right)<\frac{1}{2^{100 j_{n}}}\right\} \\
& \Omega_{n}^{2}=\left\{x \in \mathbb{R}^{3}: \operatorname{dist}\left(x, K_{n}\right)<\frac{1}{1000} \cdot \frac{1}{2^{j_{n}}}\right\} .
\end{aligned}
$$

The inequality (1.11) follows from (8.81). To show (1.12), we note that if $x \in \mathbb{R}^{3} \backslash \Omega_{n}^{2}$ and $y \in K_{n}$, then

$$
|x-y| \gtrsim 2^{-j_{n}}
$$

We can then write for $x \in \mathbb{R}^{3} \backslash \Omega_{n}^{2}$,

$$
\begin{aligned}
\left(|\nabla|^{3} \omega_{n}\right)(t, x) & =\left(|\nabla|^{-1}(-\Delta)^{2} \omega_{n}\right)(t, x) \\
& =\int_{\mathbb{R}^{3}} K(x-y) \chi_{|x-y| \gtrsim 2^{-j_{n}}}\left((-\Delta) \omega_{n}\right)(t, y) d y \\
& =\int_{\mathbb{R}^{3}} \tilde{K}(x-y) \omega_{n}(t, y) d y,
\end{aligned}
$$

where $\chi$ is a smooth cut-off function and we have integrated by parts in the last step. The modified kernel $\tilde{K}(\cdot)$ is smooth and obeys the bound

$$
|\tilde{K}(x)| \lesssim 2^{10 j_{n}}\left(1+|x|^{2}\right)^{-1}, \quad \forall x \in \mathbb{R}^{3} .
$$

Thus by (8.82) and taking $j_{n}$ sufficiently large, we obtain

$$
\begin{aligned}
& \max _{0 \leq t \leq t_{n}^{2}}\left\|\left(|\nabla|^{3} \omega_{n}\right)(t, x)\right\|_{L^{2}\left(\mathbb{R}^{3} \backslash \Omega_{n}^{2}\right)} \\
& \lesssim 2^{10 j_{n}} 2^{-100 j_{n}} \leq 1
\end{aligned}
$$

Theorem 1.10 is now proved.

\section{Ill-POSEDNESS IN BESOV SPACES}

In this last section we settle the illposedness in the Besov case.

Theorem 9.1. For any $\omega_{0}^{(g)} \in C_{c}^{\infty}\left(\mathbb{R}^{2}\right) \cap \dot{H}^{-1}\left(\mathbb{R}^{2}\right)$, any $\epsilon>0$, and any $1<p<\infty$, $1<q \leq \infty$, we can find a $C^{\infty}$ perturbation $\omega_{0}^{(p)}: \mathbb{R}^{2} \rightarrow \mathbb{R}$ such that the following hold true:

(1) The perturbation is very small:

$$
\left\|\omega_{0}^{(p)}\right\|_{L^{1}\left(\mathbb{R}^{2}\right)}+\left\|\omega_{0}^{(p)}\right\|_{L^{\infty}\left(\mathbb{R}^{2}\right)}+\left\|\omega^{(p)}\right\|_{\dot{H}^{-1}\left(\mathbb{R}^{2}\right)}+\left\|\omega_{0}^{(p)}\right\|_{B_{p, q}^{\frac{2}{p}\left(\mathbb{R}^{2}\right)}}<\epsilon .
$$

(2) Let $\omega_{0}=\omega_{0}^{(g)}+\omega_{0}^{(p)}$. The initial velocity $u_{0}=\Delta^{-1} \nabla^{\perp} \omega_{0}$ has regularity $u_{0} \in L^{2}\left(\mathbb{R}^{2}\right) \cap B_{p, q}^{1+\frac{2}{p}}\left(\mathbb{R}^{2}\right) \cap C^{\infty}\left(\mathbb{R}^{2}\right) \cap L^{\infty}\left(\mathbb{R}^{2}\right)$. 
(3) There exists a unique classical solution $\omega=\omega(t)$ to the $2 D$ Euler equation (in vorticity form)

$$
\left\{\begin{array}{l}
\partial_{t} \omega+\left(\Delta^{-1} \nabla^{\perp} \omega \cdot \nabla\right) \omega=0, \quad 0<t \leq 1, x \in \mathbb{R}^{2}, \\
\left.\omega\right|_{t=0}=\omega_{0},
\end{array}\right.
$$

satisfying $\omega(t) \in L^{1} \cap L^{\infty} \cap C^{\infty} \cap \dot{H}^{-1}, u=\Delta^{-1} \nabla^{\perp} \omega \in C^{\infty} \cap L^{2} \cap L^{\infty}$ for each $0 \leq t \leq 1$.

(4) For any $0<t_{0} \leq 1$, we have

$$
\operatorname{ess-sup}_{0<t \leq t_{0}}\|\omega(t, \cdot)\|_{\dot{B}_{p}^{\frac{2}{p}, \infty}}=+\infty .
$$

Proof of Theorem 9.1. Again with out loss of generality we assume $\omega_{0}^{(g)} \equiv 0$. We shall sketch the details and point out the important changes (as compared to the proof of Theorem (1.2). The first crucial step is the local construction. Since $B_{p, q_{1}}^{\frac{2}{p}} \hookrightarrow$ $B_{p, q_{2}}^{\frac{2}{p}}$ whenever $q_{1}<q_{2}$, it suffices for us to consider the case $B_{p, q}^{\frac{2}{p}}$ with $1<q<p$. Fix such $p$ and $q$. We will prove the following

Claim: for any small $\delta>0$, there exists a smooth initial data $\omega_{0}^{\delta} \in C_{c}^{\infty}(B(0, \delta))$ and $t_{\delta} \in(0, \delta)$ such that if $\omega^{\delta}$ is the smooth solution to

$$
\left\{\begin{array}{l}
\partial_{t} \omega^{\delta}+\left(\Delta^{-1} \nabla^{\perp} \omega^{\delta} \cdot \nabla\right) \omega^{\delta}=0, \quad 0<t \leq 1, x \in \mathbb{R}^{2} \\
\left.\omega^{\delta}\right|_{t=0}=\omega_{0}^{\delta}
\end{array}\right.
$$

then the following hold:

- $\left\|\omega_{0}^{\delta}\right\|_{B_{p, q}^{\frac{2}{p}}}+\left\|\omega_{0}^{\delta}\right\|_{L^{\infty}}+\left\|\omega_{0}^{\delta}\right\|_{\dot{H}^{-1}} \leq \delta$.

- $\operatorname{supp}\left(\omega^{\delta}(t, \cdot)\right) \subset B(0, \delta)$ for any $0 \leq t \leq \delta$.

- $\left\|\omega^{\delta}\left(t_{\delta}, \cdot\right)\right\|_{\dot{B}_{p, \infty}^{\frac{2}{p}}}>\frac{1}{\delta}$.

To prove the claim, we first take $A \gg 1$ and define one parameter of functions

$$
g_{A}^{0}=\frac{1}{\log A} \sum_{A<k<A+\log A} \eta_{k}(x),
$$

where $\eta_{k}$ is the same as in (3.1). Easy to check that

$$
\begin{gathered}
\left\|g_{A}^{0}\right\|_{\dot{B}_{r, 1}^{\frac{2}{r}}} \lesssim 1, \quad \forall 1 \leq r \leq \infty, \\
\left\|g_{A}^{0}\right\|_{\dot{B}_{\infty, \infty}^{0}} \lesssim\left\|g_{A}^{0}\right\|_{\infty} \lesssim \frac{1}{\log A} .
\end{gathered}
$$

Therefore by interpolation (choosing $r=p / q$ )

$$
\left\|g_{A}^{0}\right\|_{\dot{B}_{p, q}^{\frac{2}{p}}} \lesssim \frac{1}{(\log A)^{2 \epsilon_{1}}},
$$

where the exponent $\epsilon_{1}=\frac{1}{2}\left(1-\frac{1}{q}\right) \in\left(0, \frac{1}{2}\right)$. Now take

$$
h_{A}=(\log A)^{\epsilon_{1}} g_{A}^{0} .
$$


Obviously we have

$$
\begin{aligned}
& \left\|h_{A}\right\|_{\infty} \lesssim \frac{1}{(\log A)^{1-\epsilon_{1}}}, \\
& \left\|h_{A}\right\|_{1}+\left\|h_{A}\right\|_{\dot{H}^{-1}} \lesssim 2^{-2 A}, \\
& \left\|h_{A}\right\|_{\dot{B}_{p, q}^{\frac{2}{p}}} \lesssim \frac{1}{(\log A)^{\epsilon_{1}}} .
\end{aligned}
$$

Let $W_{A}$ be the smooth solution to the system

$$
\left\{\begin{array}{l}
\partial_{t} W_{A}+\left(\Delta^{-1} \nabla^{\perp} W_{A} \cdot \nabla\right) W_{A}=0, \quad t>0, x \in \mathbb{R}^{2}, \\
\left.W_{A}\right|_{t=0}=h_{A} .
\end{array}\right.
$$

Define the forward characteristics $\phi_{A}$ such that

$$
\left\{\begin{array}{l}
\partial_{t} \phi_{A}(t, x)=\left(\Delta^{-1} \nabla^{\perp} W_{A}\right)\left(t, \phi_{A}(t, x)\right), \\
\phi_{A}(t=0, x)=x \in \mathbb{R}^{2} .
\end{array}\right.
$$

By following the proof of Proposition 3.5 (or using Proposition 3.6, we then have for $A$ sufficiently large,

$$
M_{A}:=\max _{0 \leq t \leq \frac{1}{\log \log A}}\left\|\left(D \phi_{A}\right)(t, \cdot)\right\|_{\infty} \geq \log \log A .
$$

Clearly we can find $0<t_{A}<\frac{1}{\log \log A}$ and $x_{A}$ such that

$$
\left\|\left(D \phi_{A}\right)\left(t_{A}, x_{A}\right)\right\|_{\infty}>\frac{4}{5} M_{A} .
$$

Denote $\phi_{A}\left(t, x_{1}, x_{2}\right)=\left(\phi_{A}^{1}\left(t, x_{1}, x_{2}\right), \phi_{A}^{2}\left(t, x_{1}, x_{2}\right)\right)$. Without loss of generality we can assume

$$
\left|\left(\partial_{2} \phi_{A}^{2}\right)\left(t_{A}, x_{A}\right)\right|>\frac{4}{5} M_{A} .
$$

By continuity we can find a small neighborhood $O_{A}=B\left(x_{A}, r_{A}\right)$ of $x_{A}$ such that

$$
\left|\left(\partial_{2} \phi_{A}^{2}\right)\left(t_{A}, x\right)\right|>\frac{4}{5} M_{A}, \quad \forall x \in O_{A} .
$$

Depending on the location of $x_{A}$, we need to shrink $0<r_{A}<1$ slightly further and define an even function $b \in C_{c}^{\infty}\left(\mathbb{R}^{2}\right)$ as follows. Fix a smooth radial cut-off function $\Phi_{0} \in C_{c}^{\infty}\left(\mathbb{R}^{2}\right)$ such that $\Phi_{0}(x)=1$ for $|x| \leq \frac{1}{2}$ and $\Phi_{0}(x)=0$ for $|x|>1$. If $x_{A}=(0,0)$, we just define $b(x)=r_{A}^{-\frac{2}{p}} \Phi_{0}\left(\frac{x}{r_{A}}\right)$. If $x_{A}=\left(a_{*}, 0\right)$ for some $a_{*} \neq 0$, then we choose $r_{A}>0$ such that $r_{A} \ll\left|x_{A}\right|$. In this case we choose $b$ as an even function of $x_{1}$ and $x_{2}$ which takes the form

$$
b(x)=r_{A}^{-\frac{2}{p}}\left(\Phi_{0}\left(\frac{x-x_{A}}{r_{A}}\right)+\Phi_{0}\left(\frac{x+x_{A}}{r_{A}}\right)\right) .
$$

The case $x_{A}=\left(0, c_{*}\right)$ is similar. Now if $x_{A}=\left(a_{*}, c_{*}\right)$ with $a_{*} \neq 0$ and $c_{*} \neq 0$, then we choose $r_{A} \ll \min \left\{\left|a_{*}\right|,\left|c_{*}\right|\right\}$ and define

$$
b(x)=r_{A}{ }^{-\frac{2}{p}} \sum_{\epsilon_{1}= \pm 1, \epsilon_{2}= \pm 1} \Phi_{0}\left(\frac{x-\left(\epsilon_{1} a_{*}, \epsilon_{2} c_{*}\right)}{r_{A}}\right) .
$$

Easy to check that $b$ is an even function of $x_{1}$ and $x_{2}$. 
Therefore in all situations we can choose an even function $b \in C_{c}^{\infty}\left(\mathbb{R}^{2}\right)$ such that

$$
\begin{aligned}
& \|b\|_{L^{p}\left(O_{A}\right)} \sim 1, \\
& \|b\|_{L^{p}\left(\mathbb{R}^{2}\right)} \sim 1, \\
& \|b\|_{\dot{B}_{p, 1}^{0}\left(\mathbb{R}^{2}\right)} \lesssim 1 .
\end{aligned}
$$

In the above the implied constants depend only on the definition of the smooth cut-off function $\Phi_{0}$ and thus can be made as absolute constants. To simplify later notations and discussions, we shall still denote by $O_{A}$ the support of $b$ which are unions of even reflections of $O_{A}$ on the plane. The last inequality in (9.8) is due to the fact that translation in the real domain is equivalent to phase modulation in the frequency domain and hence

$$
\|b\|_{\dot{B}_{p, 1}^{0}} \lesssim\left\|r_{A}{ }^{-\frac{2}{p}} \Phi_{0}\left(\frac{\dot{ }}{r_{A}}\right)\right\|_{\dot{B}_{p, 1}^{0}} \lesssim\left\|\Phi_{0}\right\|_{\dot{B}_{p, 1}^{0}} \lesssim 1 .
$$

Now we consider two cases.

Case 1: $\max _{0 \leq t \leq \frac{1}{\log \log A}}\left\|W_{A}(t, \cdot)\right\|_{\dot{B}_{p}^{\frac{2}{p}}, \infty} \geq \log \log \log \log A$. In this case we set $\omega_{0}^{\delta}=W_{A}$ with $A=A(\delta)$ chosen sufficiently large. No particular work is needed in this case.

Case 2:

$$
\max _{0 \leq t \leq \frac{1}{\log \log A}}\left\|W_{A}(t, \cdot)\right\|_{\dot{B}_{p, \infty}^{\frac{2}{p}}}<\log \log \log \log A .
$$

In this case we consider

$$
\tilde{h}_{A}=h_{A}+\underbrace{\frac{1}{\log \log \log A} \cdot k^{-\frac{2}{p}} \sin \left(k x_{1}\right) \cdot b(x)}_{:=\beta(x)},
$$

where $b(x)$ was chosen as in (9.8) 17 Once again we shall take the parameter $k$ sufficiently large. Consider first $N \ll k$. Write

$$
\sin \left(k x_{1}\right) b(x)=-\frac{1}{k} \partial_{x_{1}}\left(\cos \left(k x_{1}\right) b(x)\right)+\frac{1}{k} \cos \left(k x_{1}\right) \partial_{x_{1}} b(x) .
$$

By Bernstein and the above identity, we get

$$
\begin{aligned}
N^{\frac{2}{p}}\left\|P_{N}\left(\sin \left(k x_{1}\right) b(x)\right)\right\|_{p} & \lesssim \frac{N^{1+\frac{2}{p}}}{k}\|b\|_{p}+\frac{1}{k} N^{\frac{2}{p}}\left\|P_{N}\left(\cos \left(k x_{1}\right) \partial_{x_{1}} b\right)\right\|_{p} \\
& \lesssim \frac{N^{1+\frac{2}{p}}}{k}\|b\|_{p}+\frac{1}{k} N^{\frac{2}{p}}\left\|\partial_{x_{1}} b\right\|_{p} .
\end{aligned}
$$

Summing over dyadic $N \ll k$ and letting $k$ be sufficiently large, we obtain

$$
\frac{1}{k^{\frac{2}{p}}} \sum_{N \ll k} N^{\frac{2}{p}}\left\|P_{N}\left(\sin \left(k x_{1}\right) b(x)\right)\right\|_{p} \lesssim 1 .
$$

\footnotetext{
${ }^{7}$ Of course a natural idea is to consider cutting off the high frequencies of $b$, say replacing $b$ by $b_{1}:=P_{<N_{1}} b$ for some sufficiently large $N_{1}$. This will simplify the computation of $\dot{B}_{p, 1}^{\frac{2}{p}}$ norm of the perturbation $\sin \left(k x_{1}\right) b_{1}(x)$ since for large $k \gg N_{1}$ the function $\sin \left(k x_{1}\right) b_{1}(x)$ will have frequency localized to $\{\xi:|\xi| \sim k\}$. However the disadvantage of doing this is that $b_{1}$ is not compactly supported in the $x$-space. This will bring some more unnecessary technical complications in the gluing of patch solutions later.
} 
Next consider $N \gg k$. By frequency localization, observe

$$
P_{N}\left(\sin \left(k x_{1}\right) b\right)=P_{N}\left(\sin \left(k x_{1}\right) \tilde{P}_{N} b\right),
$$

where $\tilde{P}_{N}$ is a fattened frequency projector adapted to the regime $|\xi| \sim N$. Clearly by taking $k$ sufficiently large, we have

$$
\begin{aligned}
& \frac{1}{k^{\frac{2}{p}}} \sum_{N \gg k} N^{\frac{2}{p}}\left\|P_{N}\left(\sin \left(k x_{1}\right) b(x)\right)\right\|_{p} \\
\lesssim & \frac{1}{k^{\frac{2}{p}}} \sum_{N \gg k} N^{\frac{2}{p}}\left\|\tilde{P}_{N} b\right\|_{p} \lesssim k^{-\frac{2}{p}}\|b\|_{\dot{B}_{p, 1}^{\frac{2}{p}}} \lesssim 1 .
\end{aligned}
$$

In the intermediate regime $N \sim k$, there are finitely many such dyadic $N$ and we have

$$
\frac{1}{k^{\frac{2}{p}}} \sum_{N \sim k} N^{\frac{2}{p}}\left\|P_{N}\left(\sin \left(k x_{1}\right) b(x)\right)\right\|_{p} \lesssim\|b\|_{p} \lesssim 1 .
$$

Summing over all cases, we have proved

$$
\frac{1}{k^{\frac{2}{p}}}\left\|b(x) \sin \left(k x_{1}\right)\right\|_{\dot{B}_{p, 1}^{\frac{2}{p}}} \lesssim 1 .
$$

Therefore

$$
\|\beta\|_{\dot{B}_{p, 1}^{\frac{2}{p}}} \lesssim \frac{1}{\log \log \log A}
$$

By a similar analysis, we also have

$$
\|\beta\|_{B_{r, 1}^{\frac{2}{p}}}=O(1), \quad \forall p \leq r \leq \infty .
$$

Here and below we adopt the same big $\mathrm{O}$ notation as described in the paragraph after (4.15). Denote $e_{1}=(1,0)$. Then

$$
\begin{aligned}
\left\||\nabla|^{-1} \beta\right\|_{2}^{2} & \lesssim \frac{1}{k^{\frac{4}{p}}} \int_{\mathbb{R}^{2}} \frac{1}{|\xi|^{2}} \hat{b}\left(\xi+k e_{1}\right)-\left.\hat{b}\left(\xi-k e_{1}\right)\right|^{2} d \xi \\
& \lesssim k^{-\frac{4}{p}}\left(\|x b(x)\|_{1}^{2}+\|b\|_{2}^{2}\right) \\
& =O\left(k^{-\frac{4}{p}}\right) .
\end{aligned}
$$

Therefore

$$
\|\beta\|_{\dot{H}^{-1}}=O\left(k^{-\frac{2}{p}}\right) .
$$

By (9.3) and choosing $k$ sufficiently large, we then have

$$
\begin{aligned}
& \left\|\tilde{h}_{A}\right\|_{\infty} \lesssim \frac{1}{(\log A)^{1-\epsilon_{1}}}, \\
& \left\|\tilde{h}_{A}\right\|_{1}+\left\|\tilde{h}_{A}\right\|_{\dot{H}^{-1}} \lesssim 2^{-2 A}, \\
& \left\|\tilde{h}_{A}\right\|_{\dot{B}_{p, q}^{\frac{2}{p}}} \lesssim \frac{1}{(\log A)^{\epsilon_{1}}}+\frac{1}{\log \log \log A} .
\end{aligned}
$$

Let $W_{A}^{1}$ be the smooth solution to the equation

$$
\left\{\begin{array}{l}
\partial_{t} W_{A}^{1}+\left(\Delta^{-1} \nabla^{\perp} W_{A}^{1} \cdot \nabla\right) W_{A}^{1}=0, \quad 0<t \leq 1, \\
\left.W_{A}^{1}\right|_{t=0}=\tilde{h}_{A} .
\end{array}\right.
$$


Let $\eta=W_{A}^{1}-W_{A}$ where $W_{A}$ is the solution to (9.4). Then $\eta$ satisfies

$$
\left\{\begin{array}{l}
\partial_{t} \eta+\left(\Delta^{-1} \nabla^{\perp} \eta \cdot \nabla\right) W_{A}^{1}+\left(\Delta^{-1} \nabla^{\perp} W_{A} \cdot \nabla\right) \eta=0, \\
\left.\eta\right|_{t=0}=\beta .
\end{array}\right.
$$

Now

$$
\begin{aligned}
\partial_{t}\left(\left\||\nabla|^{-1} \eta\right\|_{2}^{2}\right) \lesssim & \left\|\Delta^{-1} \nabla^{\perp} \eta\right\|_{2} \cdot\left\|W_{A}^{1}\right\|_{\infty} \cdot\left\||\nabla|^{-1} \eta\right\|_{2} \\
& +\left|\int\left(\Delta^{-1} \nabla^{\perp} W_{A} \cdot \nabla\right)\left(\Delta \Delta^{-1} \eta\right) \cdot \Delta^{-1} \eta d x\right| \\
& \lesssim\left\||\nabla|^{-1} \eta\right\|_{2}^{2} \cdot\left(\left\|W_{A}^{1}\right\|_{\infty}+\left\|\mathcal{R}_{i j} W_{A}\right\|_{\infty}\right) .
\end{aligned}
$$

Hence

$$
\max _{0 \leq t \leq 1}\left\|\left(|\nabla|^{-1} \eta\right)(t, \cdot)\right\|_{2}=O\left(k^{-\frac{2}{p}}\right) .
$$

Take $r \in(p, \infty)$. By (9.11) and local wellposedness in $B_{r, 1}^{\frac{2}{p}}$,

$$
\max _{0 \leq t \leq 1}\left(\left\|W_{A}^{1}(t, \cdot)\right\|_{B_{r, 1}^{\frac{2}{p}}}+\left\|W_{A}(t, \cdot)\right\|_{B_{r, 1}^{\frac{2}{p}}}\right)=O(1) .
$$

Interpolating the above with (9.12), we get

$$
\max _{0 \leq t \leq 1}\|\eta(t, \cdot)\|_{B_{\infty, 1}^{0}}=O\left(k^{-\alpha}\right) .
$$

Here and below we denote by the general notation $X=O\left(k^{-\alpha}\right)$ if the quantity $X \lesssim k^{-\alpha}$ for some $\alpha>0$. The value of $\alpha$ does not play much role in the analysis as long as $\alpha>0$.

Let $\Phi_{A}$ be the characteristic line associated with $W_{A}^{1}$, i.e.

$$
\left\{\begin{array}{l}
\partial_{t} \Phi_{A}(t, x)=\left(\Delta^{-1} \nabla^{\perp} W_{A}^{1}\right)\left(t, \Phi_{A}(t, x)\right), \\
\Phi_{A}(0, x)=x \in \mathbb{R}^{2} .
\end{array}\right.
$$

Set $J(t)=\left(D \Phi_{A}\right)(t)-\left(D \phi_{A}\right)(t)$. Then

$$
\begin{aligned}
& \partial_{t} J=(\left.\mathcal{R} W_{A}^{1}\right)\left(\Phi_{A}\right) J+\left(\mathcal{R}\left(W_{A}^{1}-W_{A}\right)\right)\left(\Phi_{A}\right) D \phi_{A} \\
&+\left(\left(\mathcal{R} W_{A}\right)\left(\Phi_{A}\right)-\left(\mathcal{R} W_{A}\right)\left(\phi_{A}\right)\right) D \phi_{A} .
\end{aligned}
$$

Using (9.14) and the above equation, it is easy to check

$$
\max _{0 \leq t \leq 1}\left(\left\|\left(D \Phi_{A}\right)(t, \cdot)-\left(D \phi_{A}\right)(t, \cdot)\right\|_{\infty}+\left\|\Phi_{A}(t, \cdot)-\phi_{A}(t, \cdot)\right\|_{\infty}\right)=O\left(k^{-\alpha}\right) .
$$

Let $W_{A}^{2}, W_{A}^{3}$ be the smooth solutions to the linear equations

$$
\begin{aligned}
& \left\{\begin{array}{l}
\partial_{t} W_{A}^{2}+\left(V_{A} \cdot \nabla\right) W_{A}^{2}=0, \quad t>0, \\
\left.W_{A}^{2}\right|_{t=0}=g_{A},
\end{array}\right. \\
& \left\{\begin{array}{l}
\partial_{t} W_{A}^{3}+\left(V_{A} \cdot \nabla\right) W_{A}^{3}=0, \quad t>0, \\
\left.W_{A}^{3}\right|_{t=0}=\beta,
\end{array}\right.
\end{aligned}
$$

where $V_{A}(t, x)=\left(\Delta^{-1} \nabla^{\perp} W_{A}^{1}\right)(t, x)$. Obviously,

$$
W_{A}^{1}=W_{A}^{2}+W_{A}^{3} \text {. }
$$


We first show that

$$
\max _{0 \leq t \leq 1}\left\|W_{A}(t, \cdot)-W_{A}^{2}(t, \cdot)\right\|_{\dot{B}_{p, \infty}^{\frac{2}{p}}}=O\left(k^{-\alpha}\right) .
$$

By (9.13), it is easy to check

$$
\begin{aligned}
& \max _{0 \leq t \leq 1}\left\|D^{2} W_{A}^{2}(t, \cdot)\right\|_{p}=O(1), \quad \text { if } 1<p \leq 2, \\
& \max _{0 \leq t \leq 1}\left\|D W_{A}^{2}(t, \cdot)\right\|_{p}=O(1), \quad \text { if } 2<p<\infty .
\end{aligned}
$$

On the other hand, by the Fundamental Theorem of Calculus, we have

$$
\begin{aligned}
W_{A}^{2}-W_{A} & =g_{A}\left(\tilde{\Phi}_{A}\right)-g_{A}\left(\tilde{\phi}_{A}\right) \\
& =\int_{0}^{1}\left(D g_{A}\right)\left(\tilde{\phi}_{A}+s\left(\tilde{\Phi}_{A}-\tilde{\phi}_{A}\right)\right) d s\left(\tilde{\Phi}_{A}-\tilde{\phi}_{A}\right) .
\end{aligned}
$$

Here $\tilde{\Phi}_{A}, \tilde{\phi}_{A}$ denote the inverse map of $\Phi_{A}$ and $\phi_{A}$ respectively. By (9.15) and Hölder, we then get8

$$
\begin{aligned}
& \max _{0 \leq t \leq 1}\left\|W_{A}^{2}(t, \cdot)-W_{A}(t, \cdot)\right\|_{p} \\
\lesssim & \max _{0 \leq t \leq 1}\left\|W_{A}^{2}(t, \cdot)-W_{A}(t, \cdot)\right\|_{\infty}=O\left(k^{-\alpha}\right) .
\end{aligned}
$$

Interpolating this with (9.17) then yields (9.16).

By (9.16) and (9.10), we only need to show

$$
\left\|W_{A}^{3}\left(t_{A}, \cdot\right)\right\|_{\dot{B}_{p, \infty}^{\frac{2}{p}}} \gg \log \log \log A .
$$

For this we need to introduce $W_{A}^{4}$ which solves the linear equation

$$
\left\{\begin{array}{l}
\partial_{t} W_{A}^{4}+\left(U_{A} \cdot \nabla\right) W_{A}^{4}=0, \quad 0<t \leq 1, \\
\left.W_{A}^{4}\right|_{t=0}=\sin \left(k x_{1}\right) b(x)=: W_{4,0},
\end{array}\right.
$$

where $U_{A}=\Delta^{-1} \nabla^{\perp} W_{A}$.

We shall not directly compare $W_{A}^{3}$ with $k^{-\frac{2}{p}} \frac{1}{\log \log \log A} W_{A}^{4}$ and run a perturbation argument in $\dot{B}_{p, \infty}^{\frac{2}{p}}$. Instead we will carry out an indirect argument as follows.

We first analyze the structure of $W_{A}^{4}$. Write $\tilde{\phi}_{A}=\left(\tilde{\phi}_{A}^{1}, \tilde{\phi}_{A}^{2}\right)$ and

$$
\begin{aligned}
W_{A}^{4}\left(t_{A}, x\right) & =W_{4,0}\left(\tilde{\phi}_{A}\left(t_{A}, x\right)\right) \\
& =\sin \left(k \tilde{\phi}_{A}^{1}\left(t_{A}, x\right)\right) \cdot b\left(\tilde{\phi}_{A}\left(t_{A}, x\right)\right) .
\end{aligned}
$$

Now consider

$$
F(\xi)=\int_{\mathbb{R}^{2}} \sin \left(k \tilde{\phi}_{A}^{1}\left(t_{A}, x\right)\right) b\left(\tilde{\phi}_{A}\left(t_{A}, x\right)\right) e^{-i x \cdot \xi} d x .
$$

By a change of variable $x \rightarrow \phi_{A}\left(t_{A}, x\right)$ in (9.18) (and recall that the map is volume-preserving), we get

$$
\begin{aligned}
F(\xi)= & \frac{1}{2 i} \int_{\mathbb{R}^{2}} b(x) \cdot e^{-i \phi_{A}\left(t_{A}, x\right) \cdot \xi+i k x_{1}} d x \\
& -\frac{1}{2 i} \int_{\mathbb{R}^{2}} b(x) \cdot e^{-i \phi_{A}\left(t_{A}, x\right) \cdot \xi-i k x_{1}} d x
\end{aligned}
$$

\footnotetext{
${ }^{8}$ Alternatively one can derive the estimate in an "Eulerian" way, i.e. directly derive an $L^{p}$ estimate from the equation.
} 
Consider the phase $\phi_{A}\left(t_{A}, x\right) \cdot \xi+k x_{1}$. Write

$$
\left(D \phi_{A}\right)\left(t_{A}, x\right) \xi+k\left(\begin{array}{l}
1 \\
0
\end{array}\right)=\left(D \phi_{A}\right)\left(t_{A}, x\right)\left(\xi+k\left(\left(D \phi_{A}\right)\left(t_{A}, x\right)\right)^{-1}\left(\begin{array}{l}
1 \\
0
\end{array}\right)\right) .
$$

Since $\left(\left(D \phi_{A}\right)\left(t_{A}, x\right)\right)^{-1}=\operatorname{adj}\left(\left(D \phi_{A}\right)\left(t_{A}, x\right)\right)$, by (9.6) and (9.7), we get

$$
\left.\frac{1}{C_{1}} \leq \frac{1}{M_{A}} \mid\left(\left(D \phi_{A}\right)\left(t_{A}, x\right)\right)^{-1}\left(\begin{array}{l}
1 \\
0
\end{array}\right)\right) \mid \leq C_{1}, \quad \forall x \in O_{A},
$$

where $C_{1}>0$ is an absolute constant. Now if $|\xi| \geq 2 C_{1} \cdot k M_{A}$ and $x \in O_{A}$, then

$$
\begin{aligned}
& \left|\left(D \phi_{A}\right)\left(t_{A}, x\right) \xi+k\left(\begin{array}{l}
1 \\
0
\end{array}\right)\right| \\
\gtrsim & \left|\left(\left(D \phi_{A}\right)\left(t_{A}, x\right)\right)^{-1}\right|^{-1} \cdot\left|\xi+k\left(\left(D \phi_{A}\right)\left(t_{A}, x\right)\right)^{-1}\left(\begin{array}{l}
1 \\
0
\end{array}\right)\right| \\
\gtrsim & M_{A}^{-1} \cdot|\xi| .
\end{aligned}
$$

Similarly if $|\xi| \leq \frac{1}{2 C_{1}} k M_{A}$ and $x \in O_{A}$, then

$$
\begin{aligned}
& \left|\left(D \phi_{A}\right)\left(t_{A}, x\right) \xi+k\left(\begin{array}{l}
1 \\
0
\end{array}\right)\right| \\
\gtrsim & M_{A}^{-1} \cdot k M_{A} \gtrsim k .
\end{aligned}
$$

This shows that $F(\xi)$ is essentially localized to $|\xi| \sim k M_{A}$. By (9.19), (9.20) and a stationary phase argument (note that the derivatives of $\phi_{A}$ are independent of $k !)$, we have

$$
\left\|P_{\gg k M_{A}} W_{A}^{4}\left(t_{A}, \cdot\right)\right\|_{p}+\left\|P_{\ll k M_{A}} W_{A}^{4}\left(t_{A}, \cdot\right)\right\|_{p}=O\left(k^{-\alpha}\right) .
$$

Consequently

$$
\begin{aligned}
\left\|P_{\sim k M_{A}} W_{A}^{4}\left(t_{A}, \cdot\right)\right\|_{p} & \geq\left\|W_{A}^{4}\left(t_{A}, \cdot\right)\right\|_{p}-\left\|P_{\gg k M_{A}} W_{A}^{4}\left(t_{A}, \cdot\right)\right\|_{p}-\left\|P_{\ll k M_{A}} W_{A}^{4}\left(t_{A}, \cdot\right)\right\|_{p} \\
& \geq\left\|\sin \left(k x_{1}\right) b(x)\right\|_{p}-O\left(k^{-\alpha}\right),
\end{aligned}
$$

where in the last step we have used $L^{p}$-conservation. Take an integer $m$ such that $10 p>2 m>p$, obviously for $k$ sufficiently large,

$$
\begin{aligned}
\left\|\sin \left(k x_{1}\right) b(x)\right\|_{p}^{p} & \geq \int_{\mathbb{R}^{2}}\left(\sin \left(k x_{1}\right)\right)^{2 m}|b(x)|^{p} d x \\
& \gtrsim p \int_{\mathbb{R}^{2}}\left(1-\cos \left(2 k x_{1}\right)\right)^{m}|b(x)|^{p} d x \\
& \gtrsim p\|b\|_{p}^{p}+\sum_{1 \leq j \leq m}(-1)^{j}\left(\begin{array}{c}
m \\
j
\end{array}\right) \int_{\mathbb{R}^{2}}\left(\cos \left(2 k x_{1}\right)\right)^{j}|b(x)|^{p} d x \\
& \gtrsim_{p}\|b\|_{p}^{p}+O\left(k^{-\alpha}\right) \gtrsim\|b\|_{p}^{p} \gtrsim 1 .
\end{aligned}
$$

Therefore

$$
\left\|P_{\sim k M_{A}} W_{A}^{4}\left(t_{A}, \cdot\right)\right\|_{p} \gtrsim 1
$$

Now set

$$
\eta_{1}=W_{A}^{4}-k^{\frac{2}{p}} \cdot(\log \log \log A) \cdot W_{A}^{3} .
$$


Clearly,

$$
\left\{\begin{array}{l}
\partial_{t} \eta_{1}+\left(\left(U_{A}-V_{A}\right) \cdot \nabla\right) W_{A}^{4}+\left(V_{A} \cdot \nabla\right) \eta_{1}=0 \\
\left.\eta_{1}\right|_{t=0}=0
\end{array}\right.
$$

By (9.14) (to control $U_{A}-V_{A}$ ) and a similar argument as in the derivation of (9.12), we have

$$
\max _{0 \leq t \leq 1}\left\||\nabla|^{-1} \eta_{1}(t, \cdot)\right\|_{2}=O\left(k^{-\alpha}\right) .
$$

Since $\left\|\eta_{1}\right\|_{1}+\left\|\eta_{1}\right\|_{\infty}=O(1)$, interpolation then gives

$$
\max _{0 \leq t \leq 1}\left\|\eta_{1}(t, \cdot)\right\|_{p}=O\left(k^{-\alpha}\right) .
$$

By (9.21), we then obtain

Clearly,

$$
k^{\frac{2}{p}}\left\|P_{\sim k M_{A}} W_{A}^{3}\left(t_{A}, \cdot\right)\right\|_{p} \gtrsim \frac{1}{\log \log \log A} .
$$

$$
\begin{aligned}
\left\|W_{A}^{3}\left(t_{A}, \cdot\right)\right\|_{\dot{B}_{p, \infty}^{\frac{2}{p}}} & \gtrsim\left(k M_{A}\right)^{\frac{2}{p}}\left\|P_{\sim k M_{A}} W_{A}^{3}\left(t_{A}, \cdot\right)\right\|_{p} \\
& \gtrsim \frac{M_{A}^{\frac{2}{p}}}{\log \log \log A} \\
& \gtrsim \frac{(\log \log A)^{\frac{2}{p}}}{\log \log \log A} \gg \log \log \log A .
\end{aligned}
$$

This settles Case 2 .

We have proved the claim in the local construction step.

To finish the proof of Theorem 9.1 we need to appeal to a version of Proposition 5.3 and build a solution in the form

$$
\omega(t, x)=\sum_{j=1}^{\infty} \omega_{j}(t, x)
$$

where each $\omega_{j}$ has compact support and $\operatorname{dist}\left(\operatorname{supp}\left(\omega_{j}\right), \operatorname{supp}\left(\omega_{k}\right)\right)=: R_{j k} \gg 1$ for $j \neq k$. Furthermore for any $n>1$ we can find $0<t_{n}<\frac{1}{n}$ and $j_{n}$ such that

$$
\left\|\omega_{j_{n}}\left(t_{n}, \cdot\right)\right\|_{\dot{B}_{p, \infty}^{\frac{2}{p}}}>n
$$

Due to the nonlocal nature of the Besov norm $\|\cdot\|_{\dot{B}_{p}^{\frac{2}{p}, \infty}}$, we have to control the interactions of the patches $\omega_{j}$. For this we will need to use the convexity inequality: if $1<r<\infty$ and $x, y \in \mathbb{C}^{d}$, then

$$
|x+y|^{r} \geq|x|^{r}+r|x|^{r-2} \bar{x} \cdot y, \quad \forall x, y \in \mathbb{C}^{d} .
$$

Now fix any dyadic $N \geq 2$. By the convexity inequality above, we have for any $j$,

$$
\begin{aligned}
\left\|P_{N} \omega\right\|_{p}^{p} & =\int_{\mathbb{R}^{2}}\left|P_{N} \omega_{j}+\sum_{k \neq j} P_{N} \omega_{k}\right|^{p} d x \\
& \geq\left\|P_{N} \omega_{j}\right\|_{p}^{p}+p \sum_{k \neq j} \int_{\mathbb{R}^{2}}\left|P_{N} \omega_{j}\right|^{p-2}\left(P_{N} \omega_{j}\right) \cdot P_{N} \omega_{k} d x .
\end{aligned}
$$


Observe that for any $m \geq 1, N \geq 2$,

$$
\left\|P_{N} \omega_{m}\right\|_{L^{p}\left(\left\{x: \operatorname{dist}\left(x, \operatorname{supp}\left(\omega_{m}\right)\right)>2\right\}\right)} \lesssim N^{-100}\left\|P_{N} \omega_{m}\right\|_{p} .
$$

By this and the fact $R_{j k} \gg 1$ for $j \neq k$, we have

$$
\begin{aligned}
& \left.\sum_{k \neq j}\left|\int_{\mathbb{R}^{2}}\right| P_{N} \omega_{j}\right|^{p-2}\left(P_{N} \omega_{j}\right) \cdot P_{N} \omega_{k} d x \mid \\
\lesssim & \sum_{k \neq j} N^{-100}\left\|\omega_{j}\right\|_{p}^{p-1} \cdot\left\|\omega_{k}\right\|_{p} \lesssim N^{-100},
\end{aligned}
$$

where we need to use the fact $\sum_{k=1}^{\infty}\left\|\omega_{k}\right\|_{p} \lesssim 1$ which can be easily accommodated into the construction. Clearly for any $j$,

$$
\left\|P_{N} \omega\right\|_{p}^{p} \geq\left\|P_{N} \omega_{j}\right\|_{p}^{p}-\mathrm{const} \cdot N^{-100} .
$$

From this and (9.22), it is then easy to check (9.1) holds.

Theorem 9.2. For any $\omega_{0}^{(g)} \in C_{c}^{\infty}\left(\mathbb{R}^{2}\right) \cap \dot{H}^{-1}\left(\mathbb{R}^{2}\right)$ which is odd in $x_{1}$, any $\epsilon>0$, and any $1<p<\infty, 1<q \leq \infty$, we can find a perturbation $\omega_{0}^{(p)}: \mathbb{R}^{2} \rightarrow \mathbb{R}$ such that the following hold true:

(1) $\omega_{0}^{(p)}$ is compactly supported, continuous and

$$
\left\|\omega_{0}^{(p)}\right\|_{L^{\infty}\left(\mathbb{R}^{2}\right)}+\left\|\omega^{(p)}\right\|_{\dot{H}^{-1}\left(\mathbb{R}^{2}\right)}+\left\|\omega_{0}^{(p)}\right\|_{B_{p, q}^{\frac{2}{p}\left(\mathbb{R}^{2}\right)}}<\epsilon
$$

(2) Let $\omega_{0}=\omega_{0}^{(g)}+\omega_{0}^{(p)}$. Corresponding to $\omega_{0}$, there exists a unique time-global solution $\omega=\omega(t)$ to the $2 D$ Euler equation satisfying $\omega(t) \in L^{\infty} \cap \dot{H}^{-1}$. Furthermore $\omega \in C_{t}^{0} C_{x}^{0}$ and $u=\Delta^{-1} \nabla^{\perp} \omega \in C_{t}^{0} L_{x}^{2} \cap C_{t}^{0} C_{x}^{\alpha}$ for any $0<$ $\alpha<1$.

(3) $\omega(t)$ has additional local regularity in the following sense: there exists $x_{*} \in$ $\mathbb{R}^{2}$ such that for any $x \neq x_{*}$, there exists a neighborhood $N_{x} \ni x, t_{x}>0$ such that $w(t, \cdot) \in C^{\infty}\left(N_{x}\right)$ for any $0 \leq t \leq t_{x}$.

(4) For any $0<t_{0} \leq 1$, we have

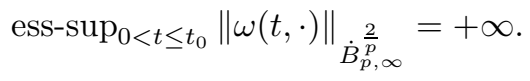

More precisely, there exist $0<t_{n}^{1}<t_{n}^{2}<\frac{1}{n}$, open precompact sets $\Omega_{n}^{1}$, $\Omega_{n}^{2}$ with $\Omega_{n}^{1} \subset \overline{\Omega_{n}^{1}} \subset \Omega_{n}^{2}, n=1,2,3, \cdots$ such that

- $\omega(t) \in C^{\infty}\left(\Omega_{n}^{2}\right)$ for all $0 \leq t \leq t_{n}^{2}$;

- $\omega(t, x) \equiv 0$ for any $x \in \Omega_{n}^{2} \backslash \Omega_{n}^{1}, 0 \leq t \leq t_{n}^{2}$.

- Define $\omega_{n}(t, x)=\omega(t, x)$ for $x \in \Omega_{n}^{1}$, and $\omega_{n}(t, x)=0$ otherwise. Then $\omega_{n} \in C_{c}^{\infty}\left(\mathbb{R}^{2}\right)$, and for some dyadic $N_{n} \geq 2$,

$$
N_{n}^{\frac{2}{p}}\left\|\left(P_{N_{n}} \omega_{n}\right)(t, \cdot)\right\|_{L^{p}\left(\mathbb{R}^{2}\right)}>n, \quad \forall t_{n}^{1} \leq t \leq t_{n}^{2},
$$

and

$$
\begin{aligned}
& \left\|\left(P_{N_{n}} \omega_{n}\right)(t, \cdot)\right\|_{L^{p}\left(x \in \mathbb{R}^{2} \backslash \Omega_{n}^{2}\right)} \\
& \quad+\left\|\left(P_{N_{n}}\left(\omega-\omega_{n}\right)\right)\right\|_{L^{p}\left(\Omega_{n}^{2}\right)} \leq \frac{1}{N_{n}^{100}}, \quad \forall 0 \leq t \leq t_{n}^{2} .
\end{aligned}
$$


Proof of Theorem 9.2. Again WLOG assume $\omega_{0}^{(g)} \equiv 0$. We shall only sketch the needed modifications (compared to the proof of Theorem 1.6 and repeating some of the steps in Theorem 9.1). In the local construction step, we take same $h_{A}$ as in (9.2). We then need to modify Lemma 6.3 (with initial data $h_{A}$ ) only slightly, namely (6.10) is replaced by

$$
\max _{0 \leq t \leq \frac{1}{\log \log \log A}}\|(D \Phi)(t, \cdot)\|_{\infty}>\log \log \log \log A .
$$

The proof stays essentially the same.

The next step in the construction is to modify Lemma 6.4 In (6.19) and the sentence before (6.21), we replace the $H^{1}$ norm by $\dot{B}_{p, q}^{\frac{2}{p}}(q=1+)$ norm. The inequality (6.21) is modified as

$$
\max _{0 \leq t \leq t_{0}}\left\|P_{>\epsilon} \frac{1}{10} \omega_{0}(t, \cdot)\right\|_{\dot{B}_{p, \infty}^{\frac{2}{p}}}>\frac{1}{\epsilon} .
$$

Also it should be noted that we need to choose $\epsilon \ll R_{0}$. One can then easily check

$$
\begin{aligned}
& \max _{0 \leq t \leq t_{0}}\left(\left\|P_{N} \omega_{0}(t, \cdot)\right\|_{L^{p}\left(x: \operatorname{dist}\left(x, \operatorname{supp}\left(\omega_{0}\right)\right) \gtrsim R_{0}\right)}\right. \\
& \left.\quad+\left\|P_{N}\left(\omega-\omega_{0}\right)(t, \cdot)\right\|_{L^{p}\left(x: \operatorname{dist}\left(x, \operatorname{supp}\left(\omega_{0}\right)\right) \lesssim R_{0}\right)}\right) \lesssim_{m} N^{-m}, \quad \forall N>\epsilon^{-\frac{1}{10}} .
\end{aligned}
$$

The last step is to glue the patch solutions. This is essentially the same as the proof of Theorem 1.6. The inequalities (9.27) and (9.28) then imply (9.25) and (9.26) respectively. To show (9.24) from (9.25) -9.26), we just decompose $\omega$ as

$$
\omega(t, x)=\omega_{n}(t, x)+g_{n}(t, x) .
$$

By (9.23),

$$
\left\|P_{N_{n}} \omega\right\|_{p}^{p} \geq\left\|P_{N_{n}} \omega_{n}\right\|_{p}^{p}-\left.p\left|\int_{\mathbb{R}^{2}}\right| P_{N_{n}} \omega_{n}\right|^{p-2}\left(P_{N_{n}} \omega_{n}\right) \cdot P_{N_{n}} g_{n} d x \mid .
$$

By construction, we have for some $R_{n}>0, \operatorname{dist}\left(\operatorname{supp}\left(\omega_{n}\right), \operatorname{supp}\left(g_{n}\right)\right)>3 R_{n}$, and

$$
\begin{aligned}
\left\|P_{N_{n}} g_{n}\right\|_{L^{p}\left(x \in \mathbb{R}^{2}: \operatorname{dist}\left(x, \operatorname{supp}\left(\omega_{n}\right)\right) \leq R_{n}\right)} \lesssim \frac{1}{N_{n}^{100}}, \\
\left\|P_{N_{n}} \omega_{n}\right\|_{L^{p}\left(x \in \mathbb{R}^{2}: \operatorname{dist}\left(x, \operatorname{supp}\left(\omega_{n}\right)\right)>R_{n}\right)} \lesssim \frac{1}{N_{n}^{100}} .
\end{aligned}
$$

Clearly,

$$
\left\|P_{N_{n}} \omega\right\|_{p}^{p} \geq\left\|P_{N_{n}} \omega_{n}\right\|_{p}^{p}-\frac{\text { const }}{N_{n}^{100}} .
$$

Thus (9.24) follows.

The last two theorems are on the illposedness of 3D Euler in Besov spaces. We omit the proof since it mimics the ones made in preceding sections.

Theorem 9.3. Consider the $3 D$ incompressible Euler equation in vorticity form:

$$
\left\{\begin{array}{l}
\partial_{t} \omega+(u \cdot \nabla) \omega=(\omega \cdot \nabla) u, \quad 0<t \leq 1, x=\left(x_{1}, x_{2}, z\right) \in \mathbb{R}^{3} \\
u=-\Delta^{-1} \nabla \times \omega \\
\left.\omega\right|_{t=0}=\omega_{0} .
\end{array}\right.
$$


For any axisymmetric vorticity $\omega_{0}^{(g)} \in C_{c}^{\infty}\left(\mathbb{R}^{3}\right)$, any $\epsilon>0$, and any $1<p<\infty$, $1<q \leq \infty$, we can find a $C^{\infty}$ perturbation $\omega_{0}^{(p)}: \mathbb{R}^{3} \rightarrow \mathbb{R}^{3}$ such that the following hold true:

(1) The perturbation is very small:

$$
\left\|\omega_{0}^{(p)}\right\|_{L^{1}\left(\mathbb{R}^{3}\right)}+\left\|\omega_{0}^{(p)}\right\|_{L^{\infty}\left(\mathbb{R}^{3}\right)}+\left\|\omega_{0}^{(p)}\right\|_{B_{p, q}^{\frac{3}{p}\left(\mathbb{R}^{3}\right)}}<\epsilon .
$$

(2) Let $\omega_{0}=\omega_{0}^{(g)}+\omega_{0}^{(p)}$. Let $u_{0}$ be the velocity corresponding to the initial vorticity $\omega_{0}$. We have $u_{0} \in B_{p, q}^{\frac{3}{p}+1}\left(\mathbb{R}^{3}\right) \cap C^{\infty}\left(\mathbb{R}^{3}\right) \cap L^{\infty}\left(\mathbb{R}^{3}\right)$.

(3) Corresponding to $\omega_{0}$, there exists a unique solution $\omega=\omega(t)$ to (9.29) on the whole time interval $[0,1]$ such that

$$
\sup _{0 \leq t \leq 1}\left(\|\omega(t, \cdot)\|_{L^{\infty}}+\|\omega(t, \cdot)\|_{L^{1}}\right)<\infty .
$$

Moreover $\omega \in C^{\infty}$ and $u \in C^{\infty}$ so that the solution is actually classical.

(4) For any $0<t_{0} \leq 1$, we have

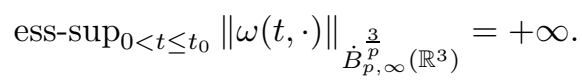

Theorem 9.4. For any axisymmetric vorticity $\omega_{0}^{(g)} \in C_{c}^{\infty}\left(\mathbb{R}^{3}\right)$, any $\epsilon>0$, and any $1<p<\infty, 1<q \leq \infty$, we can find a perturbation $\omega_{0}^{(p)}: \mathbb{R}^{3} \rightarrow \mathbb{R}^{3}$ such that the following hold true:

(1) $\omega_{0}^{(p)}$ is compactly supported, continuous and

$$
\left\|\omega_{0}^{(p)}\right\|_{L^{\infty}\left(\mathbb{R}^{3}\right)}+\left\|\omega_{0}^{(p)}\right\|_{B_{p, q}^{\frac{3}{p}}\left(\mathbb{R}^{3}\right)}<\epsilon .
$$

(2) Let $\omega_{0}=\omega_{0}^{(g)}+\omega_{0}^{(p)}$. Corresponding to $\omega_{0}$ there exists a unique solution $\omega=\omega(t, x)$ to the Euler equation (1.7) on the time interval $[0,1]$ satisfying

$$
\begin{aligned}
& \sup _{0 \leq t \leq 1}\|\omega(t, \cdot)\|_{\infty}<\infty, \\
& \operatorname{supp}(\omega(t, \cdot)) \subset\{x,|x|<R\}, \quad \forall 0 \leq t \leq 1,
\end{aligned}
$$

where $R>0$ is some constant. Furthermore $\omega \in C_{t}^{0} C_{x}^{0}$ and $u \in C_{t}^{0} L_{x}^{2} \cap$ $C_{t}^{0} C_{x}^{\alpha}$ for any $\alpha<1$.

(3) $\omega(t)$ has additional local regularity in the following sense: there exists $x_{*} \in$ $\mathbb{R}^{3}$ such that for any $x \neq x_{*}$, there exists a neighborhood $N_{x} \ni x, t_{x}>0$ such that $w(t) \in C^{\infty}\left(N_{x}\right)$ for any $0 \leq t \leq t_{x}$.

(4) For any $0<t_{0} \leq 1$, we have

$$
\operatorname{ess-sup}_{0<t \leq t_{0}}\|\omega(t, \cdot)\|_{\dot{B}_{p, \infty}^{\frac{3}{p}}\left(\mathbb{R}^{3}\right)}=+\infty .
$$

\section{REFERENCES}

[1] H. Abidi, T. Hmidi and S. Keraani. On the global well-posedness for the axisymmetric Euler equations. Math. Ann. 347 (2010), no. 1, 15-41.

[2] C. Bardos and E. Titi. Loss of smoothness and energy conserving rough weak solutions for the $3 d$ Euler equations. Discrete Contin. Dyn. Syst. Ser. S 3 (2010), no. 2, 185-197.

[3] J.T. Beale, T. Kato and A. Majda. Remarks on the breakdown of smooth solutions for the 3D Euler equations. Comm. Math. Phys. 94(1984) 61-66.

[4] J.P. Bourguignon and H. Brezis. Remarks on the Euler equation. J. Functional Analysis 15 (1974), 341-363.

[5] J.-Y. Chemin. Perfect incompressible fluids. Clarendon press, Oxford, 1998. 
[6] D. Chae. Local existence and blow-up criterion for the Euler equations in the Besov spaces. Asymptot. Anal. 38 (2004), no. 3-4, 339-358.

[7] P. Constantin. On the Euler equations of incompressible fluids. Bull. Amer. Math. Soc. (N.S.) 44 (2007), no. 4, 603-621.

[8] A. Cheskidov and R. Shvydkoy. Ill-posedness of the basic equations of fluid dynamics in Besov spaces. Proc. Amer. Math. Soc. 138 (2010), no. 3, 1059-1067.

[9] R. Danchin: Axisymmetric incompressible flows with bounded vorticity. Russian Math. Surveys 62 (2007), no 3, p. 73-94.

[10] D. Ebin and J. Marsden. Groups of diffeomorphisms and the motion of an incompressible fluid. Ann. of Math. (2) 921970 102-163.

[11] R. DiPerna and A. Majda. Oscillations and concentrations in weak solutions of the incompressible fluid equations. Comm. Math. Phys. 108 (1987), no. 4, 667-689.

[12] N. Gunther, On the motion of fluid in a moving container, Izvestia Akad. Nauk USSR, Ser. Fiz.-Mat., Vol. 20, 1927, pp.1323-1348, 1503-1532; Vol. 21, 1927, pp. 521-526, 735-756; Vol. 22, 1928, pp. 9-30.

[13] A.A. Himonas and G. Misiołek. Non-uniform dependence on initial data of solutions to the Euler equations of hydrodynamics. Comm. Math. Phys. 296 (2010), no. 1, 285-301.

[14] H. Inci. On the well-posedness of the incompressible Euler equation. arXiv: 1301.5997.

[15] T. Kato. Nonstationary flows of viscous and ideal fluids in $\mathbb{R}^{3}$. J. Functional Analysis 9 (1972), 296-305.

[16] T. Kato. The Cauchy problem for quasi-linear symmetric hyperbolic systems. Arch. Rational Mech. Anal. 58 (1975), no. 3, 181-205.

[17] T. Kato and G. Ponce. Commutator estimates and the Euler and Navier-Stokes equations. Comm. Pure Appl. Math. 41 (1988), no. 7, 891-907.

[18] L. Lichtenstein, Üer einige Existenzprobleme der Hydrodynamik homogenerunzusammendrück barer, reibungsloser Flüßbigkeiten und die Helmgoltzschen Wirbelsatze, Math. Z., Vol. 23, 1925, pp. 89-154; Vol. 26, 1927, pp. 196-323; Vol. 28, 1928, pp. 387-415; Vol. 32, 1930, pp. 608-725.

[19] O. A. Ladyzhenskaya: Unique global solvability of the three-dimensional Cauchy problem for the Navier-Stokes equations in the presence of axial symmetry. Zapisky Nauchnych Sem. LOMI 7 (1968), p. 155-177.

[20] P.L. Lions. Mathematical topics in fluid mechanics. Volume 1. "Incompressible Models". Oxford Lecture Series in Mathematics and Its Apllications. The Clarendon Press, Oxford University Press, New York, 1996.

[21] A. Majda and A. Bertozzi, Vorticity and incompressible flow, Cambridge Texts in Applied Mathematics, 27. Cambridge University Press, Cambridge, 2002.

[22] G. Misiołek. and T. Yoneda. Ill-posedness examples for the quasi-geostrophic and the Euler equations. Analysis, geometry and quantum field theory, 251-258, Contemp. Math., 584, Amer. Math. Soc., Providence, RI, 2012.

[23] H.C. Pak and Y.J. Park. Existence of solution for the Euler equations in a critical Besov space $B_{\infty, 1}^{1}\left(\mathbb{R}^{d}\right)$. Comm. Partial Differential Equations 29 (2004), no. 7-8, 1149-1166.

[24] T. Shirota, T. Yanagisawa: Note on global existence for axially symmetric solutions of the Euler system. Proc. Japan Acad. Ser. A Math. Sci. 70 (1994), no. 10, p. 299-304.

[25] R. Takada. Counterexamples of commutator estimates in the Besov and the Triebel-Lizorkin spaces related to the Euler equations. SIAM J. Math. Anal. 42 (2010), no. 6, 2473-2483.

[26] M. R. Ukhovskii, V. I. Yudovich: Axially symmetric flows of ideal and viscous fluids filling the whole space. Prikl. Mat. Meh. 32 (1968), no. 1, p. 59-69.

[27] M. Vishik. Hydrodynamics in Besov spaces. Arch. Rat. Mech. and Anal., Vol. 145, 1998, pp. 197-214.

[28] M. Vishik. Incompressible flows of an ideal fluid with vorticity in borderline spaces of Besov type. Ann. Sci. École Norm. Sup. (4) 32 (1999), no. 6, 769-812.

[29] W. Wolibner. Un theorème sur l'existence du mouvement plan d'un fluide parfait, homogène, incompressible, pendant un temps infiniment long. Math. Z. 37 (1933), no. 1, 698-726.

[30] V. Yudovich, Nonstationary flow of an ideal incompressible liquid, Zh. Vych. Mat., Vol. 3, 1963, pp. 1032-1066.

[31] V. Yudovich, Uniqueness theorem for the basic nonstationary problem in the dynamics of an ideal incompressible fluid. Mathematical Research Letters, Vol. 2, 1995, pp. 27-38. 
(J. Bourgain) School of Mathematics, Institute for Advanced Study, Princeton, NJ 08544, USA

E-mail address: bourgain@math.ias.edu

(D. Li) Department of Mathematics, University of British Columbia, Vancouver BC CANAdA V6T 122

E-mail address: mpdongli@gmail.com 